JAMA Internal Medicine | Original Investigation

\title{
Effect of Fish Oil Supplementation and Aspirin Use on Arteriovenous Fistula Failure in Patients Requiring Hemodialysis A Randomized Clinical Trial
}

\author{
Ashley B. Irish, MD; Andrea K. Viecelli, MD; Carmel M. Hawley, MD, MMedSci; Lai-Seong Hooi, MD; Elaine M. Pascoe, MBiostat; \\ Peta-Anne Paul-Brent, BSc; Sunil V. Badve, MD; Trevor A. Mori, PhD; Alan Cass, MD, PhD; Peter G. Kerr, MD, PhD; \\ David Voss, MD; Loke-Meng Ong, MD; Kevan R. Polkinghorne, MD, PhD; for the Omega-3 Fatty Acids (Fish Oils) \\ and Aspirin in Vascular Access Outcomes in Renal Disease (FAVOURED) Study Collaborative Group
}

IMPORTANCE Vascular access dysfunction is a leading cause of morbidity and mortality in patients requiring hemodialysis. Arteriovenous fistulae are preferred over synthetic grafts and central venous catheters due to superior long-term outcomes and lower health care costs, but increasing their use is limited by early thrombosis and maturation failure. $\omega-3$ Polyunsaturated fatty acids (fish oils) have pleiotropic effects on vascular biology and inflammation and aspirin impairs platelet aggregation, which may reduce access failure.

OBJECTIVE To determine whether fish oil supplementation (primary objective) or aspirin use (secondary objective) is effective in reducing arteriovenous fistula failure.

DESIGN, SETTING, AND PARTICIPANTS The Omega-3 Fatty Acids (Fish Oils) and Aspirin in Vascular Access Outcomes in Renal Disease (FAVOURED) study was a randomized, double-blind, controlled clinical trial that recruited participants with stage 4 or 5 chronic kidney disease from 2008 to 2014 at 35 dialysis centers in Australia, Malaysia, New Zealand, and the United Kingdom. Participants were observed for 12 months after arteriovenous fistula creation.

INTERVENTIONS Participants were randomly allocated to receive fish oil $(4 \mathrm{~g} / \mathrm{d})$ or matching placebo. A subset $(n=406)$ was also randomized to receive aspirin $(100 \mathrm{mg} / \mathrm{d})$ or matching placebo. Treatment started 1 day prior to surgery and continued for 12 weeks.

MAIN OUTCOMES AND MEASURES The primary outcome was fistula failure, a composite of fistula thrombosis and/or abandonment and/or cannulation failure, at 12 months. Secondary outcomes included the individual components of the primary outcome.

RESULTS Of 1415 eligible participants, 567 were randomized (359 [63\%] male, 298 [53\%] white, 264 [47\%] with diabetes; mean [SD] age, 54.8 [14.3] y). The same proportion of fistula failures occurred in the fish oil and placebo arms (128 of 270 [47\%] vs 125 of 266 [47\%]; relative risk [RR] adjusted for aspirin use, $1.03 ; 95 \% \mathrm{Cl}, 0.86-1.23 ; P=.78)$. Fish oil did not reduce fistula thrombosis (60 [22\%] vs 61 [23\%]; RR, 0.98; $95 \% \mathrm{Cl}, 0.72-1.34 ; P=.90$ ), abandonment (51 [19\%] vs 58 [22\%]; RR, 0.87; 95\% Cl, 0.62-1.22; $P=.43$ ), or cannulation failure (108 [40\%] vs 104 [39\%]; RR, 1.03; 95\% Cl, 0.83-1.26; $P=.81$ ). The risk of fistula failure was similar between the aspirin and placebo arms ( 87 of 194 [45\%] vs 83 of 194 [43\%]; RR, 1.05; 95\% Cl, 0.84-1.31; $P=.68)$.

CONCLUSIONS AND RELEVANCE Neither fish oil supplementation nor aspirin use reduced failure of new arteriovenous fistulae within 12 months of surgery.

TRIAL REGISTRATION anzctr.org.au Identifier: CTRN12607000569404

JAMA Intern Med. 2017;177(2):184-193. doi:10.1001/jamainternmed.2016.8029 Published online January 3, 2017.
Editor's Note page 194

Supplemental content at jamainternalmedicine.com
Author Affiliations: Author affiliations are listed at the end of this article.

Group Information: The Omega-3 Fatty Acids (Fish Oils) and Aspirin in Vascular Access Outcomes in Renal Disease (FAVOURED) Study Collaborative Group members are listed at the end of this article.

Corresponding Author: Ashley B. Irish, MD, Department of Renal Medicine, Fiona Stanley Hospital, 102-118 Murdoch Dr, Murdoch, Western Australia 6150, Australia (ashley.irish@health.wa.gov.au). 
emodialysis is the commonest renal replacement modality worldwide and requires a functioning permanent vascular access. Vascular access dysfunction is associated with substantial morbidity and mortality and presents a major health economic burden. ${ }^{1,2}$ Arteriovenous fistulae (AVFs) are recommended as the preferred vascular access by international clinical practice guidelines ${ }^{3-6}$ because of their longevity and lower complication rates compared with synthetic arteriovenous grafts (AVGs) and central venous catheters. ${ }^{2,7,8}$ However, AVFs require a longer maturation time before use and have a significantly higher risk of early failure compared with AVGs. ${ }^{9}$ Early primary failure occurs in $20 \%$ to $50 \%$ of AVFs as a result of either thrombosis or maturation failure. ${ }^{10,11}$ Although antiplatelet agents can reduce early thrombosis of AVFs, this has not translated into significant improvements in the proportion of AVFs that become suitable for dialysis. ${ }^{12,13}$

Supplementation of $\omega-3$ polyunsaturated fatty acids found in fish oil may be beneficial in promoting vascular access maturation and reducing thrombosis by not only inhibiting platelet aggregation ${ }^{14,15}$ but also decreasing blood viscosity, improving red blood cell flexibility, ${ }^{16,17}$ promoting vasodilation, ${ }^{18}$ inhibiting smooth muscle cell proliferation, ${ }^{19}$ and reducing inflammation. ${ }^{20,21}$ Fish oil has been shown to increase patency rates in AVGs, but it has not been assessed in AVFs. ${ }^{22,23}$ Low-dose aspirin is an effective antiplatelet agent in the secondary prevention of vascular disease ${ }^{24}$ and has been used safely in patients with advanced kidney disease ${ }^{25}$; however, it has not been adequately evaluated in the prevention of AVF failure.

The primary aim of the Omega-3 Fatty Acids (Fish Oils) and Aspirin in Vascular Access Outcomes in Renal Disease (FAVOURED) trial was to test the hypothesis that fish oil supplementation will reduce AVF failure after AVF creation in patients with stage 4 or 5 chronic kidney disease. A secondary aim was to examine the efficacy of aspirin use in preventing failure of new AVFs (registration ACTRN12607000569404).

\section{Methods}

\section{Study Design}

FAVOURED is an investigator-initiated, international, doubleblind, randomized, placebo-controlled trial conducted in 35 hemodialysis centers across Australia, Malaysia, New Zealand, and the United Kingdom (study protocol and statistical analysis plan in Supplement 1). Recruitment began on August 21, 2008, and ended on February 28, 2014, with the last follow-up on February 28, 2015. The original study, which commenced with a 2 by 2 factorial design, was amended in June 2011. First, the primary outcome of "early thrombosis" at 12 weeks following AVF creation was broadened to the more clinically relevant "AVF access failure," a composite of thrombosis and/or AVF abandonment and/or cannulation failure at 12 months. Second, eligibility criteria were broadened so that patients who were previously ineligible due to medically indicated aspirin use were eligible and randomized to fish oil or matching placebo with continuation of open-label aspirin (eFig-

\section{Key Points}

Question Is fish oil or aspirin effective in preventing failure of de novo arteriovenous fistulae in patients requiring hemodialysis?

Findings In this randomized clinical trial that included 567 adults, the occurrence of fistula failure within 12 months of surgery was high and not reduced by fish oil supplementation or aspirin use compared with placebo.

Meaning This study suggests that neither fish oil nor aspirin can be recommended routinely for the prevention of arteriovenous fistula failure and that additional strategies to reduce the high arteriovenous fistula failure rate are required.

ure 1 in Supplement 2). As a result of the second amendment, the primary objective of FAVOURED was to assess the efficacy of fish oil supplementation on AVF failure at 12 months and the efficacy of aspirin was assessed as a secondary objective. Details of the original and amended study protocols have been published previously. ${ }^{26-28}$

\section{Study Oversight}

The study was approved by human research ethics committees at participating centers. All patients provided written consent prior to study enrolment. A trial management committee comprising clinicians and a statistician had sole responsibility for the design and conduct of the study, analysis of data, and manuscript preparation. An independent data and safety monitoring board reviewed accumulating data. Two interim efficacy analyses using the Haybittle-Peto rule were planned after one-third and two-thirds of patients had been recruited and observed for at least 12 months. Due to early cessation of recruitment, only the first interim analysis was performed, after which the study continued as planned until terminated because of slower than anticipated accrual, funding issues, and lack of ongoing availability of trial medications. FAVOURED is registered with the Australia and New Zealand Clinical Trials Register (ACTRN12607000569404).

\section{Study Population}

Adults (age $>19$ years) with stage 4 or 5 chronic kidney disease receiving or planned to receive hemodialysis within 12 months and scheduled for AVF surgery in the arm were eligible for inclusion. Participants were excluded if they had an increased bleeding risk (bleeding disorder, recent or active gastrointestinal ulcer, platelet count $<100 \times 10^{3} / \mu \mathrm{L}$ [to convert to billions per liter, multiply by 1.0$]$, hepatic insufficiency), were taking aspirin within 2 weeks or fish oil within 4 weeks of trial commencement, were taking nonsteroidal anti-inflammatory drugs, anticoagulants, or antiplatelet agents, or had contraindications for taking the study agents. In June 2011, the exclusion criterion of current use of aspirin was removed so that patients currently taking aspirin were eligible for recruitment (eFigure 1 in Supplement 2).

\section{Randomization and Study Intervention}

Randomization of participants was dependent on aspirintaking status: (1) not taking aspirin or able to cease; (2) taking 
aspirin and unable to cease (open-label aspirin). Within each group, participants were randomly assigned in a 1:1 ratio to receive either $4 \mathrm{~g}$ of fish oil ( $2 \mathrm{~g}$ twice daily) in the form of $4 \omega$-3acid ethyl esters (Omacor) capsules (46\% eicosapentaenoic acid and $38 \%$ docosahexaenoic acid) or 4 matching placebo capsules (olive oil) (BGP Products Pty Ltd, trading as Mylan EPD). Participants not taking aspirin or able to cease were further randomized in a 1:1 ratio to receive $100 \mathrm{mg}$ of oral aspirin daily or matching placebo (Bayer Healthcare). Randomization was performed by a central, web-based system (Flexetrials) using an adaptive minimization algorithm with study site and planned location of the AVF (upper vs lower arm) as minimization variables. Randomization occurred up to 7 days prior to scheduled AVF surgery. Treatment commenced on the day before scheduled AVF surgery and continued for 12 weeks. Participants, caregivers, treating physicians and surgeons, laboratory staff, and members of the study team were blinded to treatment allocation.

\section{Outcomes}

The primary outcome was AVF access failure at 12 months after fistula creation and defined as a composite of any one of AVF thrombosis, AVF abandonment, and cannulation failure. Thrombosis was defined as the absence of a thrill or bruit by clinical assessment and/or requirement for rescue intervention (medical thrombolysis or surgical thrombectomy). Arteriovenous fistula abandonment was defined as no further use of, or attempt to intervene on, the study AVF. Cannulation failure was defined as failure to successfully cannulate the study AVF during 8 or more of 12 consecutive hemodialysis sessions during the cannulation assessment period ${ }^{28}$ (eTable 1 in Supplement 2). Secondary outcomes included components of the primary outcome, and safety outcomes.

\section{Sample Size}

The study was designed to detect a $30 \%$ relative reduction in AVF access failure assuming $30 \%$ AVF failure in control groups (an absolute risk reduction of $9 \%$ ). At $80 \%$ power and a 2 -sided significance level of 5\%, 734 participants would be required (367 per group). Assuming a 5\% drop-in from control to fish oil and a 5\% drop-out from fish oil to placebo and a 5\% loss to follow-up, a total of 954 participants needed to be recruited.

\section{Statistical Analysis}

Log binomial regression (to enable calculation of relative risks $[R R s]^{29}$ ) was used to compare fish oil with matching placebo on the composite outcome of AVF access failure, adjusted for differences in aspirin use (randomized to aspirin, randomized to placebo aspirin, open-label aspirin). The treatment effect is expressed as RR and 95\% confidence interval. The robustness of the fish oil treatment effect was assessed by additional analyses that adjusted for prespecified baseline characteristics and study region (eFigure 2 in Supplement 2). All prespecified subgroup analyses were performed using log binomial regression models adjusted for differences in aspirin use. Tests of treatment-by-subgroup variable interactions informed the interpretation of subgroup results. Secondary outcomes were analyzed using log binomial regression models as per the primary outcome. Single imputation was used to replace missing values on the separate components of the primary outcome as follows: participants with missing data were randomly allocated a value ( $1=$ event, 0 = no event) such that the proportion of imputed events was the same as the proportion of events in participants with observed data for the component; for example, if the overall frequency of AVF thrombosis for participants with available data was $20 \%$, then a patient with a missing value for AVF thrombosis would be randomly assigned an event in $20 \%$ of cases. A range of prespecified sensitivity analyses for imputation of missing values were performed, including a complete case analysis. Comparisons of aspirin with matching placebo were performed using the same statistical models.

Analyses were based on participants' randomized treatment. Participants who did not receive AVF creation surgery or died within 12 months of surgery and were not assessed on any of the 3 components of the primary outcome before their death were excluded. A 2 -sided $P<.05$ was considered statistically significant. Statistical analyses were performed using SAS version 9.4 (SAS Institute) and Stata version 13.1 (Stata Corporation).

\section{Results}

\section{Study Participants}

Of the 4242 patients assessed for eligibility, 1415 were deemed eligible, 567 were randomized to fish oil $(n=284)$ or placebo $(\mathrm{n}=283$ ), and 536 included in the analysis (Figure 1). There were 406 participants randomized to aspirin $(n=203)$ or matching placebo $(n=203)$. Participant demographic and clinical characteristics were well balanced between each pair of treatment groups (Table 1).

\section{AVF Failure}

Arteriovenous fistula failure occurred in 128 of 270 (47\%) participants assigned to fish oil and 125 of 266 (47\%) assigned to placebo (RR adjusted for aspirin use, 1.03; 95\% CI, 0.86-1.23; $P=.78$ ). The proportion of events in the fish oil and placebo groups was similar for each of the 3 individual components of AVF failure: thrombosis (60 [22\%] vs 61 [23\%]; adjusted RR, 0.98; 95\% CI, 0.72-1.34; $P=.90)$, AVF abandonment (51 [19\%] vs 58 [22\%]; RR, 0.87; 95\% CI, 0.62-1.22; $P=.43$ ), and cannulation failure during the cannulation assessment period (108 [40\%] vs 104 [39\%]; RR, 1.03; 95\% CI, 0.83-1.26; $P=.81$ ) (Table 2). In the assessment of the prespecified subgroups, a significant difference in the effect of fish oil on AVF failure was observed in participants with diabetes mellitus compared with those without diabetes $(P=.03$ for interaction; RR, 1.30; 95\% CI, 0.99-1.71 vs RR, 0.85; 95\% CI, 0.67-1.08), but not in other prespecified subgroups (Figure $2 \mathrm{~A}$ ).

In participants randomized to aspirin or matching placebo, the risk of AVF failure was similar (87 [45\%] vs 83 [43\%]; RR, 1.05; 95\% CI, 0.84-1.31; $P=.68)$. Aspirin compared with placebo did not reduce the risk of AVF thrombosis (38 [20\%] vs 35 [18\%]; RR, 1.09; 95\% CI, 0.72-1.64; $P=.70)$, AVF abandonment (46 [24\%] vs 35 [18\%]; RR, 1.31; 95\% CI, 0.89-1.95; 
$P=.17$ ), or cannulation failure (73 [38\%] vs 74 [38\%]; RR, 0.99; 95\% CI, 0.76-1.27; $P$ = .92) (Table 2). There were no statistically significant subgroup effects involving the aspirin groups (Figure 2B). The fish oil by aspirin interaction test was not statistically significant for the composite outcome $(P=.12)$; however, results for comparisons of fish oil plus aspirin with fish oil and aspirin alone and double placebo are included in eTable 2 in Supplement 2.

Analyses based on complete cases and secondary analyses adjusting for prespecified baseline characteristics and/or geographic region demonstrated results comparable to those of the primary analyses for both fish oil and aspirin (eFigure $2 \mathrm{~A}$ and B in Supplement 2). The amounts of missing data (23\% for fish oil and placebo; $22 \%$ for aspirin and $21 \%$ for placebo) and number of imputed events were similar between treatment groups (eTable 3 in Supplement 2). The majority of "missing" data were due to 81 of 122 (66\%) participants who did not start dialysis within 12 months of surgery and thus could not be assessed for cannulation failure.

Post hoc analyses examining the timing of first cannulation (eTable 4 in Supplement 2), the time from fistula creation to initiation of hemodialysis (eTable 5 in Supplement 2), and the timing of renal replacement therapy initiation in predialysis participants (eTable 6 in Supplement 2) showed no differences between treatment groups.

\section{Adverse Events}

Adverse drug events, including bleeding events (16 [6\%] vs 10 [4\%]; $P=.23$ ) and gastrointestinal adverse effects (15 [5\%] vs 14 [5\%]; $P=.86$ ), were similar for the fish oil and placebo groups. Death occurred in 8 participants allocated to fish oil and 9 allocated to placebo (Table 3). Aspirin use was not associated with an increase in adverse drug events or serious adverse events (Table 3).

\section{Adherence}

After 6 and 12 weeks of treatment, the percentage of eicosapentaenoic acid and docosahexaenoic acid incorporated in erythrocytes (measured in Australian and New Zealand participants taking fish oil) doubled, but it remained at baseline levels for those taking placebo, confirming adherence to study treatment and providing evidence that the fish oil formulation was sufficient to modify lipid composition of erythrocytes (eFigure 3 in Supplement 2). For all participants, the median number of returned capsules was comparable for both the fish oil and placebo groups (47 vs 45 of 360 dispensed capsules) and the aspirin and placebo groups (7 of 90 dispensed tablets for both groups).

\section{Discussion}

In patients with chronic kidney disease undergoing creation of an AVF, 3 months' treatment with fish oil at a dose of $4 \mathrm{~g}$ per day did not reduce the composite primary outcome of AVF failure nor any of the individual components, which included AVF abandonment, thrombosis, and cannulation failure, assessed at 12 months. The trial finished before reaching
Figure 1. Consolidated Standards of Reporting Trials Diagram: Study Enrollment and Follow-up

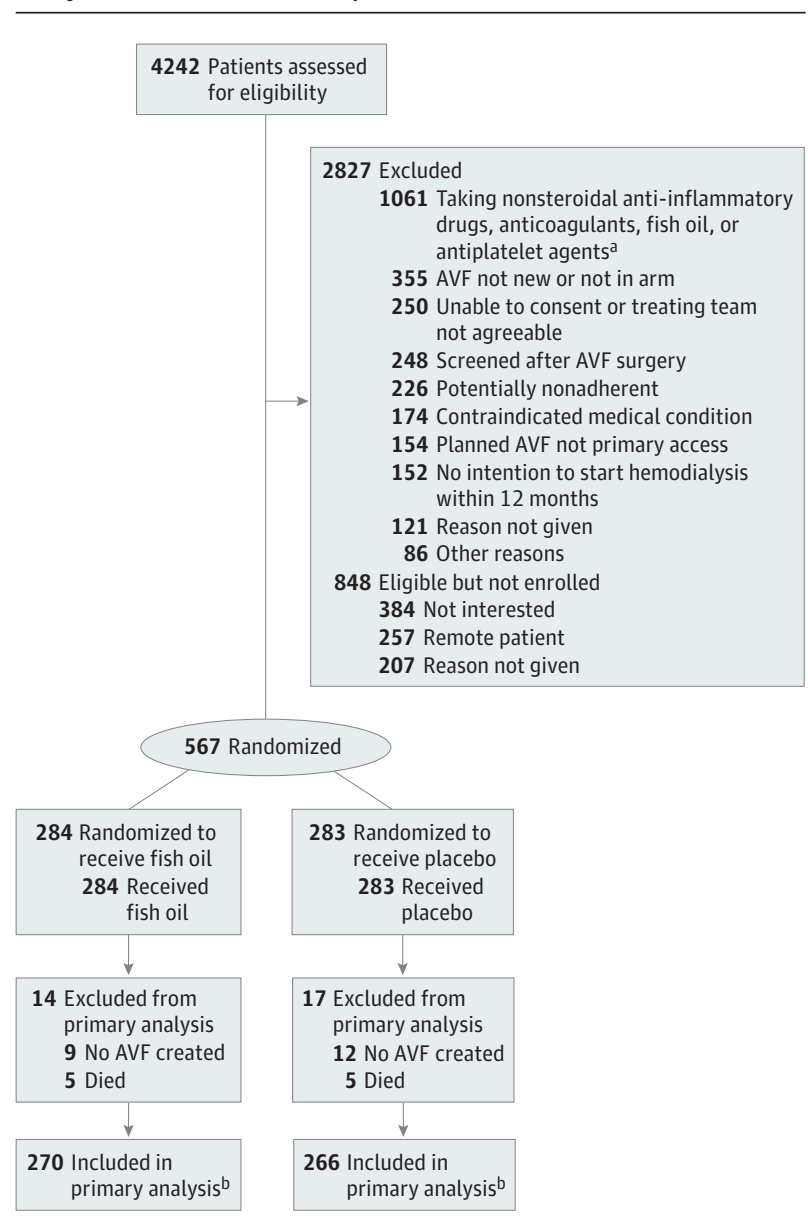

${ }^{\text {a }}$ Following the protocol amendment in 2011, participants unable to discontinue aspirin use were eligible for randomization to fish oil or matching placebo with continuation of open-label aspirin use.

${ }^{\mathrm{b}}$ Number of participants whose primary outcome was determined by imputation; fish oil $\mathrm{n}=62(23 \%)$, placebo $\mathrm{n}=60(23 \%)$.

AVF indicates arteriovenous fistula.

the planned recruitment target but was able to show that fish oil is unlikely to reduce the risk of AVF failure by $30 \%$; results were consistent with, at best, a $14 \% \mathrm{RR}$ reduction. In a smaller subset of patients, the use of low-dose aspirin was similarly ineffective in improving AVF outcomes. This large, international trial confirmed the high AVF failure of nearly $50 \%$ at 12 months after surgery and emphasizes the pressing need to improve AVF outcomes.

Previous studies regarding the benefits of fish oil supplementation in vascular access outcomes have been confined to AVGs, in which the results are conflicting. Lok and colleagues ${ }^{22}$ found no significant difference in the primary outcome measure, proportion of patency loss of newly created AVGs within 12 months, in 201 patients treated with $4 \mathrm{~g}$ of fish oil or placebo. However, the study demonstrated improvements in secondary outcomes including rates of patency loss, interventions, and thrombosis in addition to cardiovascular benefits. One small randomized clinical trial of 24 participants treated 


\begin{tabular}{|c|c|c|c|c|}
\hline Characteristic & $\begin{array}{l}\text { Fish Oil } \\
(\mathrm{n}=284)\end{array}$ & $\begin{array}{l}\text { Placebo } \\
(\mathrm{n}=283)\end{array}$ & $\begin{array}{l}\text { Aspirin } \\
(\mathrm{n}=203)\end{array}$ & $\begin{array}{l}\text { Placebo } \\
(\mathrm{n}=203)\end{array}$ \\
\hline Age, mean (SD), y & $54.1(14.0)$ & $55.6(14.5)$ & $52.3(14.5)$ & $53.8(14.9)$ \\
\hline Male sex, No. (\%) & $180(63)$ & $179(63)$ & $125(62)$ & $131(65)$ \\
\hline \multicolumn{5}{|l|}{ Country, No. (\%) } \\
\hline Australia and New Zealand & $202(71)$ & $201(71)$ & $150(74)$ & $147(72)$ \\
\hline Malaysia & $79(28)$ & $76(27)$ & $53(26)$ & $53(26)$ \\
\hline United Kingdom & $3(1)$ & $6(2)$ & 0 & $3(2)$ \\
\hline \multicolumn{5}{|l|}{ Ethnicity, No. (\%) } \\
\hline Asian & $92(32)$ & $89(32)$ & $68(34)$ & $61(30)$ \\
\hline White & $144(51)$ & $154(55)$ & $107(53)$ & $119(59)$ \\
\hline Indigenous $^{\mathrm{a}}$ & $39(14)$ & $27(10)$ & $24(12)$ & $15(7)$ \\
\hline Other & $9(3)$ & $11(4)$ & $4(2)$ & $7(4)$ \\
\hline BMI, mean (SD) & $28.8(7.4)$ & $28.4(7.2)$ & $27.9(6.9)$ & $28.3(7.7)$ \\
\hline \multicolumn{5}{|l|}{ Blood pressure, mean (SD), $\mathrm{mm} \mathrm{Hg}$} \\
\hline Systolic & $146(23)$ & $146(23)$ & $145(22)$ & $146(24)$ \\
\hline Diastolic & $82(14)$ & $81(13)$ & $81(13)$ & $83(14)$ \\
\hline \multicolumn{5}{|l|}{ Comorbid conditions, No. (\%) } \\
\hline Diabetes mellitus & $139(49)$ & $125(45)$ & $83(41)$ & $75(37)$ \\
\hline Ischemic heart disease & $30(11)$ & $28(10)$ & $9(4)$ & $3(2)$ \\
\hline Congestive heart disease & $10(4)$ & $12(4)$ & $6(3)$ & $3(2)$ \\
\hline Peripheral vascular disease & $14(5)$ & $10(4)$ & $6(3)$ & $5(3)$ \\
\hline Cerebrovascular disease & $6(2)$ & $11(4)$ & $2(1)$ & $2(1)$ \\
\hline Hypertension & $247(87)$ & $255(91)$ & $183(90)$ & $179(89)$ \\
\hline Current or prior smoking & $155(55)$ & $135(48)$ & $99(49)$ & $95(47)$ \\
\hline \multicolumn{5}{|l|}{ Medications, No. (\%) } \\
\hline Aspirin & $78(28)$ & $78(28)$ & NA & NA \\
\hline Statin & $152(54)$ & $143(51)$ & $85(42)$ & $93(46)$ \\
\hline Erythropoietin-stimulating agent & $125(44)$ & $141(50)$ & $104(51)$ & $92(46)$ \\
\hline$\beta$-Blocker & $130(46)$ & $133(47)$ & $94(46)$ & $82(41)$ \\
\hline $\begin{array}{l}\text { Angiotensin receptor blocker or } \\
\text { angiotensin-converting enzyme inhibitor }\end{array}$ & $118(42)$ & $122(43)$ & $91(45)$ & $82(41)$ \\
\hline Calcium channel blocker & $158(56)$ & $159(56)$ & $118(58)$ & $110(55)$ \\
\hline Intravenous iron & $49(17)$ & $49(17)$ & $39(19)$ & $43(21)$ \\
\hline Xanthine-oxidase inhibitor & $50(18)$ & $38(13)$ & $32(16)$ & $28(14)$ \\
\hline \multicolumn{5}{|l|}{ Etiology of renal disease, №. (\%) } \\
\hline Diabetic nephropathy & $124(44)$ & $97(35)$ & $70(35)$ & $56(28)$ \\
\hline Glomerulonephritis & $38(13)$ & $39(14)$ & $29(14)$ & $33(16)$ \\
\hline Hypertension/vascular & $37(13)$ & $38(14)$ & $16(8)$ & $37(18)$ \\
\hline Polycystic kidney disease & $18(6)$ & $22(8)$ & $20(10)$ & $18(9)$ \\
\hline Reflux nephropathy & $15(5)$ & $11(4)$ & $11(5)$ & $14(7)$ \\
\hline Other & $52(18)$ & $74(26)$ & $57(28)$ & $44(22)$ \\
\hline \multicolumn{5}{|l|}{$\begin{array}{l}\text { Planned study AVF location, } \\
\text { No. }(\%)\end{array}$} \\
\hline Upper arm & $113(40)$ & $110(39)$ & $84(41)$ & $82(40)$ \\
\hline Forearm & $171(60)$ & $173(61)$ & $119(59)$ & $121(60)$ \\
\hline \multicolumn{5}{|l|}{$\begin{array}{l}\text { Renal replacement therapy at time } \\
\text { of AVF creation, No. (\%) }\end{array}$} \\
\hline Peritoneal dialysis & $15(5)$ & $20(7)$ & $17(8)$ & $11(5)$ \\
\hline Hemodialysis & $122(43)$ & $118(42)$ & $82(40)$ & $92(46)$ \\
\hline Not currently receiving dialysis & $147(52)$ & $143(51)$ & $104(51)$ & $99(49)$ \\
\hline
\end{tabular}




\begin{tabular}{|c|c|c|c|c|}
\hline Characteristic & $\begin{array}{l}\text { Fish Oil } \\
(\mathrm{n}=284)\end{array}$ & $\begin{array}{l}\text { Placebo } \\
(\mathrm{n}=283)\end{array}$ & $\begin{array}{l}\text { Aspirin } \\
(n=203)\end{array}$ & $\begin{array}{l}\text { Placebo } \\
(n=203)\end{array}$ \\
\hline \multicolumn{5}{|l|}{$\begin{array}{l}\text { Principal access currently in use for participants } \\
\text { receiving dialysis, No. (\%) }\end{array}$} \\
\hline AVF & $5(4)$ & $2(2)$ & $2(2)$ & $1(1)$ \\
\hline Arteriovenous graft & 0 & $1(1)$ & 0 & 0 \\
\hline Central venous catheter, cuffed and noncuffed & $117(85)$ & $115(83)$ & $80(81)$ & $91(88)$ \\
\hline Peritoneal dialysis catheter & $15(11)$ & $20(15)$ & $17(17)$ & $11(11)$ \\
\hline Dialysis duration, ${ }^{\mathrm{C}}$ median (IQR), mo & $3.8(1.8-18.2)$ & $4.2(1.9-17.7)$ & $4.2(2.0-16.3)$ & $3.9(1.8-15.4)$ \\
\hline Hemoglobin concentration, mean (SD), g/dL & $10.9(1.8)$ & $10.8(1.9)$ & $10.9(1.9)$ & $10.8(1.9)$ \\
\hline Serum albumin level, mean (SD), g/dL & $3.60(0.62)$ & $3.60(0.63)$ & $3.61(0.63)$ & $3.63(0.60)$ \\
\hline eGFR, ${ }^{c}$ mean $(S D), m L / m i n / 1.73 \mathrm{~m}^{2}$ & $11.2(6.3)$ & $11.4(5.1)$ & $9.4(4.9)$ & $9.6(6.2)$ \\
\hline \multicolumn{2}{|c|}{$\begin{array}{l}\text { Abbreviations: AVF, arteriovenous fistula; BMI, body mass index, calculated as } \\
\text { weight in kilograms divided by height in meters squared; eGFR, estimated } \\
\text { glomerular filtration rate; IQR, interquartile range; NA, not applicable. }\end{array}$} & \multicolumn{3}{|c|}{$\begin{array}{l}\text { b Actual AVF location upper arm vs forearm: fish oil } n=117(42 \%) \text { vs } n=162 \\
(58 \%) \text {; placebo } n=110(40 \%) \text { vs } n=163(60 \%) \text {; aspirin } n=83(41 \%) \text { vs } \\
n=118(59 \%) \text {; placebo } n=82(41 \%) \text { vs } n=116(59 \%) \text {. }\end{array}$} \\
\hline \multicolumn{2}{|c|}{$\begin{array}{l}\text { SI conversion factor: To convert hemoglobin or albumin to grams per liter, } \\
\text { multiply by } 10 \text {. }\end{array}$} & \multicolumn{3}{|c|}{$\begin{array}{l}\text { ' } \text { For predialysis participants only (fish oil } n=147 \text { and placebo } n=143 \text {; aspirin } \\
n=104 \text { and placebo } n=99 \text { ). }\end{array}$} \\
\hline
\end{tabular}

Table 2. Primary and Secondary Outcomes for Fish Oil vs Placebo and Aspirin vs Placebo

\begin{tabular}{|c|c|c|c|c|c|}
\hline \multirow[b]{2}{*}{ Outcome ${ }^{a}$} & \multicolumn{4}{|l|}{ №. (\%) } & \multirow{9}{*}{$\begin{array}{l}\text { Abbreviations: AVF, arteriovenous } \\
\text { fistula; RR, relative risk. }\end{array}$} \\
\hline & Active & Placebo & $\mathrm{RR}(95 \% \mathrm{Cl})$ & $P$ Value & \\
\hline \multicolumn{5}{|l|}{ Fish Oil $(n=270)$ vs Placebo $(n=266)$} & \\
\hline \multicolumn{5}{|l|}{ Primary outcome } & \\
\hline AVF failure ${ }^{b}$ & $128(47)$ & $125(47)$ & $1.03(0.86-1.23)$ & $.78^{c}$ & \\
\hline \multicolumn{5}{|l|}{ Secondary outcomes } & \\
\hline \multirow{3}{*}{$\begin{array}{l}\text { AVF abandonment within } 12 \text { mo } \\
\text { Thrombosis of study AVF within } 12 \\
\text { Cannulation failure within } 12 \text { mo }\end{array}$} & $51(19)$ & $58(22)$ & $0.87(0.62-1.22)$ & .43 & \\
\hline & $60(22)$ & $61(23)$ & $0.98(0.72-1.34)$ & .90 & \\
\hline & $108(40)$ & $104(39)$ & $1.03(0.83-1.26)$ & .81 & \\
\hline \multicolumn{5}{|l|}{ Aspirin $(n=194)$ vs Placebo $(n=194)$} & \multirow{7}{*}{$\begin{array}{l}{ }^{\text {a }} \text { Adjusted for differences in aspirin } \\
\text { use (no aspirin, randomized to } \\
\text { aspirin, open-label aspirin). } \\
{ }^{\text {b } C o m p o s i t e ~ o f ~ t h r o m b o s i s, ~ A V F ~} \\
\text { abandonment, and cannulation } \\
\text { failure. } \\
\text { C Interaction } P=.12 \text { for additivity of } \\
\text { fish oil and aspirin effect on } \\
\text { composite outcome. }\end{array}$} \\
\hline \multicolumn{5}{|l|}{ Primary outcome } & \\
\hline AVF failure ${ }^{b}$ & $87(45)$ & $83(43)$ & $1.05(0.84-1.31)$ & .68 & \\
\hline \multicolumn{5}{|l|}{ Secondary outcomes } & \\
\hline AVF abandonment within $12 \mathrm{mo}$ & $46(24)$ & $35(18)$ & $1.31(0.89-1.95)$ & .17 & \\
\hline Thrombosis of study AVF within 12 mo & $38(20)$ & $35(18)$ & $1.09(0.72-1.64)$ & .70 & \\
\hline Cannulation failure within $12 \mathrm{mo}$ & $73(38)$ & $74(38)$ & $0.99(0.76-1.27)$ & .92 & \\
\hline
\end{tabular}

with $4 \mathrm{~g}$ of fish oil or placebo had a 12-month patency of $76 \%$ in the fish oil-treated group compared with $15 \%$ in the placebo group, ${ }^{23}$ which was not confirmed in another small randomized trial using lower doses of eicosapentaenoic and docosahexaenoic acids. ${ }^{30}$ Variability in these results may be due to differences in sample size, and dose and duration of therapy.

While participants receiving fish oil during the FAVOURED trial had increased erythrocyte $\omega-3$ polyunsaturated fatty acids, supporting medication adherence, a clinically relevant effect of fish oil on AVF failure was not demonstrated. It remains unknown whether the lack of efficacy is due to achieved levels of eicosapentaenoic and docosahexaenoic acids being insufficient to promote adequate vasodilatory, antiproliferative, anti-aggregatory, and anti-inflammatory effects at the site of the AVF formation to improve clinical outcomes. However, the doses of eicosapentaenoic and docosahexaenoic acids used in the FAVOURED trial were higher than doses used in studies assessing AVG outcomes and studies in patients with chronic kidney disease using a comparable dose of fish oil achieved significant reductions in blood viscosity, platelet aggregation, and blood pressure consistent with biological effects. ${ }^{15,16,31}$ Three months of therapy was selected because it corresponds with the expected maturation time and early failure of AVF. ${ }^{32,33}$ However, the possibility that a longer duration of treatment may have resulted in a delayed benefit on maturation and usability cannot be excluded. It is also possible that risk factors such as age, diabetes mellitus, cardiovascular disease, and peripheral vascular disease that affect characteristics of the conduit vesse ${ }^{34,35}$ may limit any beneficial effects of fish oil supplementation on AVF failure. Although there was no difference in AVF failure between the fish oil and placebo groups, participants with diabetes mellitus had a higher risk of AVF failure when treated with fish oil than those without diabetes mellitus. The biological plausibility of this finding is uncertain, although differences in life- 
Figure 2. Forest Plot for Prespecified Subgroup Analysis on Arteriovenous Fistula (AVF) Failure

\begin{tabular}{|c|c|c|}
\hline $\begin{array}{l}\text { A Fish oil vs placebo } \\
\text { Subgroup }\end{array}$ & $\begin{array}{l}\text { Events/No. of } \\
\text { Patients (\%) }\end{array}$ & $\mathrm{RR}(95 \% \mathrm{Cl})$ \\
\hline \multicolumn{3}{|l|}{$\overline{\text { Sex }}$} \\
\hline Male & $148 / 342(43)$ & $0.98(0.77-1.25)$ \\
\hline Female & $105 / 194(54)$ & $1.07(0.83-1.39)$ \\
\hline \multicolumn{3}{|l|}{ Age, y } \\
\hline$\leq 45$ & $56 / 135(41)$ & $1.00(0.67-1.50)$ \\
\hline$\leq 56$ & $54 / 130(42)$ & $0.96(0.64-1.43)$ \\
\hline$\leq 65$ & $70 / 135(52)$ & $1.06(0.78-1.44)$ \\
\hline$>65$ & $73 / 136(54)$ & $1.02(0.73-1.42)$ \\
\hline \multicolumn{3}{|l|}{ Diabetes } \\
\hline No & $142 / 293(48)$ & $0.85(0.67-1.08)$ \\
\hline Yes & $111 / 243(46)$ & $1.30(0.99-1.71)$ \\
\hline \multicolumn{3}{|l|}{ Cardiovascular disease $\mathrm{a}^{\mathrm{a}}$} \\
\hline No & $212 / 457(46)$ & $1.01(0.83-1.23)$ \\
\hline Yes & $41 / 79(52)$ & $1.11(0.71-1.73)$ \\
\hline \multicolumn{3}{|l|}{ Dialysis $^{\mathrm{b}}$} \\
\hline No & $126 / 277(45)$ & $1.00(0.77-1.29)$ \\
\hline Yes & $127 / 259(49)$ & $1.06(0.84-1.35)$ \\
\hline \multicolumn{3}{|l|}{ Planned AVF site } \\
\hline Lower arm & $152 / 329(46)$ & $1.02(0.81-1.29)$ \\
\hline Upper arm & $101 / 207$ (49) & $1.04(0.79-1.36)$ \\
\hline \multicolumn{3}{|l|}{ Actual AVF site } \\
\hline Lower arm & $149 / 319(47)$ & $1.12(0.89-1.42)$ \\
\hline Upper arm & $104 / 217(48)$ & $0.91(0.69-1.21)$ \\
\hline
\end{tabular}

B Aspirin vs placebo

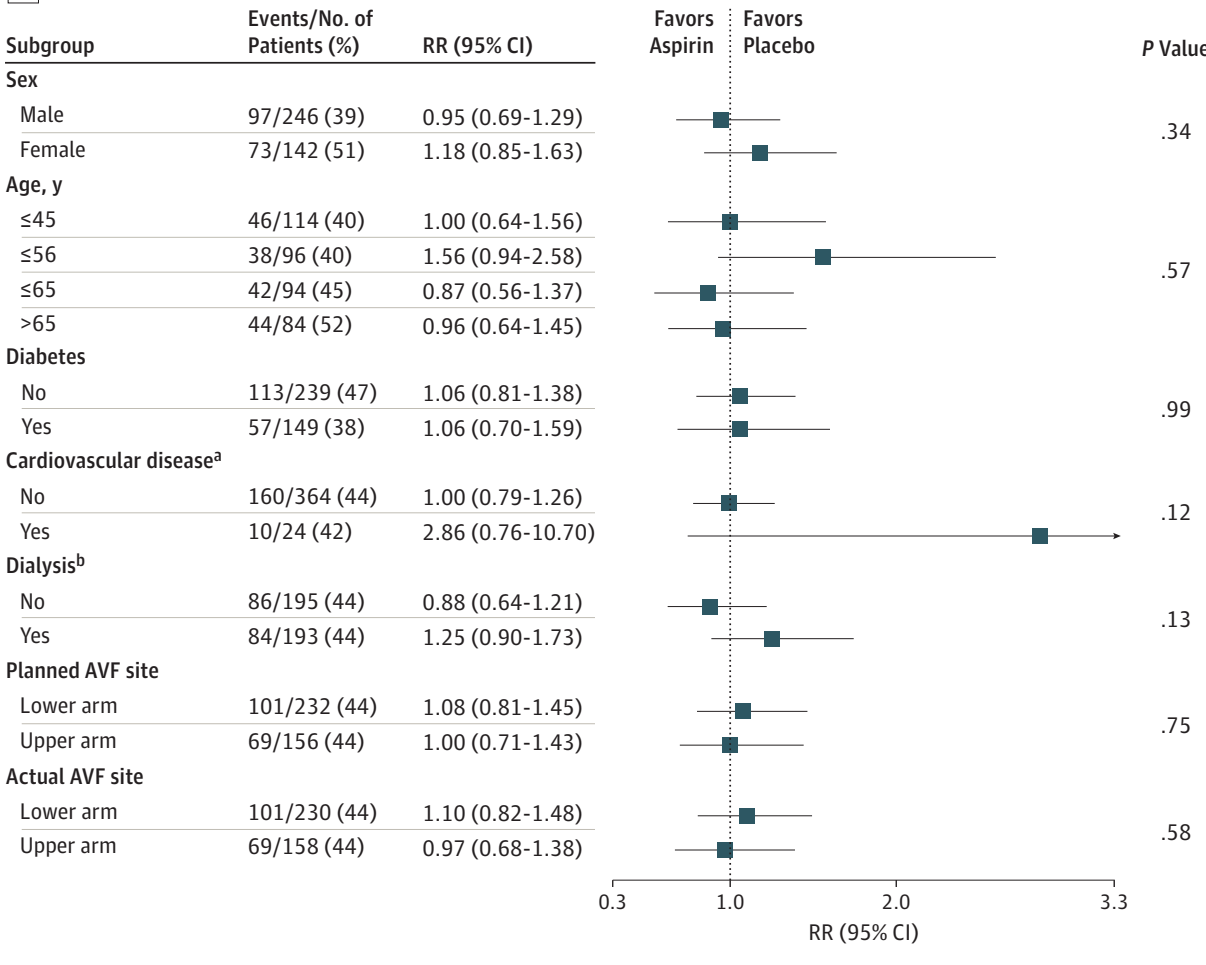

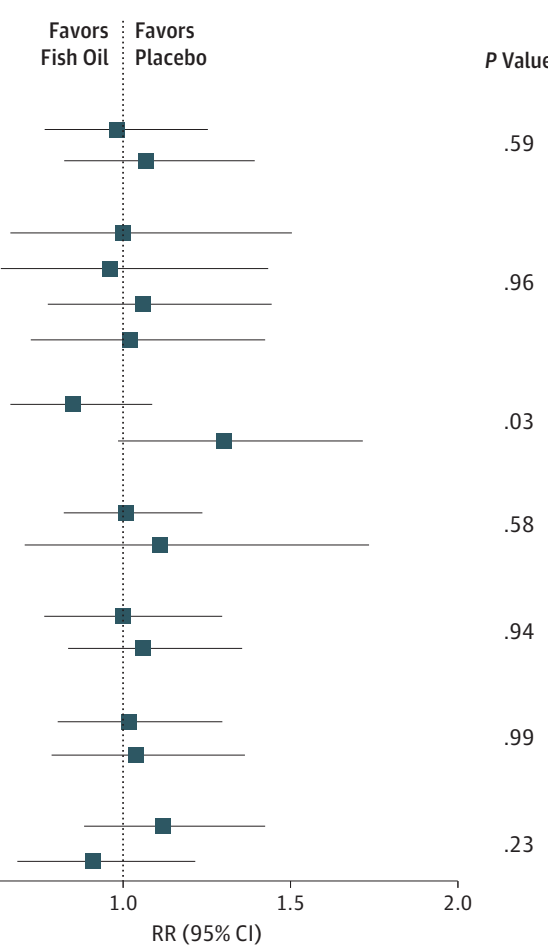

Value 34

RR indicates relative risk.

${ }^{a}$ Includes any 1 or more of peripheral vascular disease, ischemic heart disease, and cerebrovascular disease.

${ }^{\mathrm{b}}$ No indicates predialysis/transplant: yes, hemodialysis or peritoneal dialysis. style factors associated with diabetic status or a type I statistical error are possible explanations.

Aspirin is a well-established antiplatelet agent with wide clinical efficacy in preventing vascular thrombotic events. ${ }^{24}$
One small randomized clinical trial had previously shown a beneficial effect of aspirin use on early AVF thrombosis using $1 \mathrm{~g}$ of aspirin every other day for 28 days. ${ }^{36}$ In our study, 3 months of low-dose aspirin use did not reduce AVF failure or 
Table 3. Adverse Events Fish Oil vs Placebo and Aspirin vs Placebo

\begin{tabular}{|c|c|c|c|}
\hline \multirow[b]{2}{*}{ Adverse Event ${ }^{a}$} & \multicolumn{2}{|l|}{ No. (\%) } & \multirow[b]{2}{*}{$P$ Value } \\
\hline & Active & Placebo & \\
\hline \multicolumn{4}{|l|}{ Fish Oil $(n=284)$ vs Placebo $(n=283)$} \\
\hline \multicolumn{4}{|l|}{ Adverse drug events } \\
\hline Bleeding event & $16(6)$ & $10(4)$ & .23 \\
\hline Gastrointestinal event & $15(5)$ & $14(5)$ & .86 \\
\hline Other event & $8(3)$ & $6(2)$ & .59 \\
\hline \multicolumn{4}{|l|}{ Severe adverse events } \\
\hline Death & $8(3)$ & $9(3)$ & .80 \\
\hline Life-threatening event & $2(1)$ & $4(1)$ & .41 \\
\hline Hospitalization & $108(38)$ & 109 (39) & .91 \\
\hline Disability & $1(<1)$ & $1(<1)$ & $>.99$ \\
\hline Other important medical event & $2(1)$ & $6(2)$ & .15 \\
\hline \multicolumn{4}{|c|}{ Aspirin $(n=203)$ vs Placebo $(n=203)$} \\
\hline \multicolumn{4}{|l|}{ Adverse drug events } \\
\hline Bleeding event & $12(6)$ & $12(6)$ & $>.99$ \\
\hline Gastrointestinal event & $13(6)$ & $10(5)$ & .52 \\
\hline Other event & $6(3)$ & $4(2)$ & .52 \\
\hline \multicolumn{4}{|l|}{ Severe adverse events } \\
\hline Death & $7(3)$ & $4(2)$ & .36 \\
\hline Life-threatening event & $2(1)$ & $3(2)$ & .65 \\
\hline Hospitalization & $81(40)$ & $72(36)$ & .36 \\
\hline Disability & 0 & $1(1)$ & $\ldots$ \\
\hline Other important medical event & $4(2)$ & $3(2)$ & .70 \\
\hline
\end{tabular}

adverse events included events occurring within 6 months of arteriovenous fistula creation (ie, those plausibly related to study). thrombosis. In contrast, 6 weeks of clopidogrel bisulfate therapy reduced early access thrombosis from $20 \%$ to $12 \%$ although this did not increase the proportion of usable AVF. ${ }^{13}$ The lower dose of aspirin used in FAVOURED may have been inadequate for thrombosis prevention.

Consistent with contemporary international studies, ${ }^{9,13,37,38}$ we report $47 \%$ AVF failure. Both thrombosis and failure to mature were unaffected by the interventions at 12 months, suggesting that modifying vascular conduit function to improve vascular access outcomes requires additional research focus. This high failure rate remains the most important impediment to successful hemodialysis and is considered a critical priority by patients and clinicians. ${ }^{39} \mathrm{~A}$ substantial proportion of patients undergoing fistula creation with the expectation of requiring hemodialysis did not commence dialysis within 12 months of surgery. This uncertainty in predicting dialysis commencement and timing of AVF surgery in patients with advanced chronic kidney disease may have implications for both resource use and future trial considerations. ${ }^{40}$

To our knowledge, FAVOURED is the first trial evaluating fish oil supplementation and the largest trial evaluating aspirin use and AVF outcomes. Relevant clinical end points to define vascular access failure were included, and participants were from diverse health care settings and geographic regions. Treatment groups were well balanced with respect to baseline characteristics.

\section{Limitations}

The trial population was younger with less vascular disease than anticipated because we excluded patients with medical requirements for antiplatelet agents or anticoagulants. There were missing data on the primary outcome, mainly because patients had not commenced dialysis and therefore could not be assessed for cannulation failure; however, the proportion of missing values was balanced across treatment groups and imputation is unlikely to have biased the results.

\section{Conclusions}

The proportion of new AVFs that failed within 12 months of surgery was nearly $50 \%$ and was not reduced by 12 weeks of fish oil supplementation or low-dose aspirin therapy. This study suggests that neither fish oil nor aspirin can be recommended for the prevention of AVF failure and that additional strategies to reduce the high AVF failure rate are required.

\section{ARTICLE INFORMATION}

Accepted for Publication: October 19, 2016.

Published Online: January 3, 2017 doi:10.1001/jamainternmed.2016.8029

Author Affiliations: Department of Nephrology, Fiona Stanley Hospital, Perth, Australia (Irish);
School of Medicine and Pharmacology, University of Western Australia, Perth, Australia (Irish, Mori); Department of Nephrology, Princess Alexandra Hospital, Brisbane, Australia (Viecelli, Hawley); Australasian Kidney Trials Network, School of Medicine, University of Queensland, Brisbane, Australia (Viecelli, Hawley, Pascoe, Paul-Brent,
Badve); Department of Medicine and Hemodialysis Unit, Hospital Sultanah Aminah, Johor Bahru, Malaysia (Hooi); Department of Nephrology, St George Hospital, Sydney, Australia (Badve); The George Institute for Global Health, Sydney, Australia (Badve); Menzies School of Health Research,

Charles Darwin University, Darwin, Australia (Cass); 
Department of Nephrology, Monash Medical Centre, Melbourne, Australia (Kerr, Polkinghorne); Department of Medicine, Monash University, Melbourne, Australia (Kerr, Polkinghorne); Middlemore Renal Department, Counties-Manukau Health, Auckland, New Zealand (Voss); Department of Nephrology, Penang Hospital, Georgetown, Malaysia (Ong); School of Public Health and Preventive Medicine, Monash University, Melbourne, Australia (Polkinghorne).

Author Contributions: Dr Hawley and Ms Pascoe had full access to all of the data in the study and take responsibility for the integrity of the data and the accuracy of the data analysis. Drs Irish and Viecelli are co-first authors, with equal contribution to the manuscript.

Study concept and design: Irish, Hawley, Pascoe, Paul-Brent, Mori, Cass, Kerr, Polkinghorne. Acquisition, analysis, or interpretation of data: Irish, Viecelli, Hawley, Hooi, Pascoe, Paul-Brent, Badve Mori, Kerr, Voss, Ong, Polkinghorne. Drafting of the manuscript: Irish, Viecelli, Hawley, Hooi, Paul-Brent, Badve, Voss, Polkinghorne. Critical revision of the manuscript for important intellectual content: Irish, Viecelli, Hawley, Hooi, Pascoe, Badve, Mori, Cass, Kerr, Voss, Ong, Polkinghorne.

Statistical analysis: Hawley, Pascoe, Badve. Obtained funding: Irish, Hawley, Paul-Brent, Mori, Kerr, Polkinghorne.

Administrative, technical, or material support Viecelli, Hawley, Hooi, Pascoe, Paul-Brent, Mori, Kerr, Voss, Ong, Polkinghorne.

Study supervision: Irish, Hawley, Hooi, Pascoe, Badve, Mori, Cass, Kerr, Voss, Polkinghorne.

Group Information: The FAVOURED Study Collaborative Group comprises the Trial Steering Committee: Chen Au Peh, CNARTS, Royal Adelaide Hospital, Adelaide, Australia; Elaine Beller, Faculty of Health Services and Medicine, Bond University, Gold Coast, Australia; Alan Cass, Menzies School of Health Research, Darwin, Australia; Sharan Dogra, Department of Renal Medicine, Sir Charles Gairdner Hospital, Perth, Australia; David Gracey, Department of Renal Medicine, Royal Prince Alfred Hospital, Sydney, Australia; Elvie Haluszkiewicz, Department of Vascular Surgery, Royal Perth Hospital, Perth, Australia; Carmel Hawley, Department of Nephrology, Princess Alexandra Hospital, Brisbane, Queensland, Australia; LaiSeong Hooi, Hemodialysis Unit, Hospital Sultanah Aminah, Johor Bahru, Malaysia; Colin Hutchison, Renal Services, Hawkes Bay Hospital, Hawke's Bay, New Zealand; Ashley Irish, Department of Nephrology Fiona Stanley Hospital, Perth, Western Australia, Australia; Peter Kerr, Department of Nephrology, Monash Medical Centre, Melbourne, Australia; Amanda Mather, Royal North Shore Hospital, Sydney, Australia; Stephen McDonald, CNARTS, Royal Adelaide Hospital, Adelaide, Australia; Chris McIntyre, London Health Sciences Centre, Ontario, Canada; Trevor Mori, Medicine and Pharmacology RPH Unit, University of Western Australia, Perth, Australia; Elaine Pascoe, Australasian Kidney Trials Network, University of Queensland, Brisbane, Australia; Kevan Polkinghorne, Department of Nephrology, Monash Medical Centre, Melbourne, Victoria, Australia; Amanda Robertson, Nephrology Surgery, Royal Melbourne Hospital, Melbourne, Australia; Johan Rosman, Medicine and Pharmacology RPH Unit, University of Western Australia, Perth, Australia;
David Voss, Middlemore Renal Department, Counties-Manukau Health, Auckland, New Zealand.

The Data and Safety Monitoring Board: Andrew Tonkin and Andrew Forbes, Department of Epidemiology and Preventive Medicine, Monash University, Melbourne, Australia; Adeera Levin, Department of Medicine, University of British Columbia, Vancouver, Canada; David C. Wheeler, Center for Nephrology, Royal Free and University College Medical School, London, England.

The Investigators: Australia: Australian Capital Territory: Canberra Hospital, Krishna Karpe, Patricia Johnson; New South Wales: Concord Repatriation General Hospital, Martin Gallagher, Jenny Burman; John Hunter Hospital, Alistair Gillies, Leanne Garvey; Liverpool Hospital, Michael Suranyi, Belinda Yip; Prince of Wales Hospital, Grant Luxton, Debbie Pugh, Kathleen McNamara; Royal North Shore Hospital, Bruce Cooper, Cheryl Macadam Royal Prince Alfred Hospital, Kate Wyburn, Samantha Hand; Sydney Adventist Hospital, Meg Jardine, Anne Heath; Queensland: Gold Coast Hospital, Dakshinamurthy Divi, Tammy Schmidt; Greenslopes Private Hospital, Andrew Bofinger, Leanne Glancy; Princess Alexandra Hospital, David Mudge, Amanda Coburn; Toowoomba Hospital, Sree Krishna Venuthurpali, Elizabeth Coroneos, Suzi Hanna; Townsville Hospital, George Kan, Vicki Hartig; South Australia: Royal Adelaide Hospital, Karen Katil, Meg Hockley; Victoria: Austin Health, Peter Mount, Pascal Bisscheroux, Maree RossSmith; Geelong Hospital, Rob McGinley, Anthony Perkins; Monash Medical Centre, Kevan Polkinghorne, Mechelle Seneviratne; Royal Melbourne Hospital, Eugenie Pedagogos, Connie Karchimkus; Alfred Hospital, Solomon Menahem, Suzanne Douglas; Western Health, Vicki Levidiotis, Debra Broomfield, Jason Bennier; Western Australia: Fremantle Hospital, Paolo Ferrari, Ulrich Steinweadel; Royal Perth Hospital, Ashley Irish, Maria Martin, Monika Chang; Sir Charles Gairdner Hospital, Sharan Dogra, Susan Pellicano, Helen Herson; Malaysia: Kuala Lumpur Hospital, Ravindran Visvanathan, Norlida Omar, Zanariah Arsat; Pulau Pinang Hospital, Loke-Meng Ong, Ah-Heong Ang; Raja Perempuan Zainab II Hospital, Sukeri Mohamad, Najhah Md Nawi; Seberang Jaya Hospital, Anita Bhajan Manocha, Norhaniza Bt Adom; Sultanah Aminah General Hospital, LaiSeong Hooi, Wen Jiun Liu, Mohd Rais Bin Periman, Norisham Bin Mohd Dom; Sultanah Nur Zahirah Hospital, Zawawi Nordin, Suhaizan Bt Mohd Rasidi; Taiping Hospital, Indralingam Vaithilingam, Ramli Zaraee; University Malaya Medical Centre, Lim Soo Kun, Tiviyah Sinniah; New Zealand: Dunedin Hospital, John Schollum, Liz Berry; CountiesManukau Health, David Voss, Penelope Eadie United Kingdom: Royal Derby Hospital, Chris McIntyre, Marie Appleby.

The Australasian Kidney Trials Network Executive Committee Members: Neil Boudville, Department of Renal Medicine, Sir Charles Gairdner Hospital, Perth, Australia; Alan Cass, Menzies School of Health Research, Darwin, Australia; Carmel Hawley, Department of Nephrology, Princess Alexandra Hospital, Brisbane, Australia; Meg Jardine, George Institute of Global Health, Sydney, Australia; David Johnson, Department of Nephrology, Princess Alexandra Hospital, Brisbane, Australia; Vlado Perkovic, George Institute of Global Health, Sydney, Australia.
The Australasian Kidney Trials Network Project Management Team: Carmel Hawley, David Johnson, Alicia Morrish, Elaine Pascoe, Peta-Anne Paul-Brent, Donna Reidlinger, Liza Vergara, Australasian Kidney Trials Network, University of Queensland, Brisbane, Australia; Elaine Beller, Faculty of Health Services and Medicine, Bond University, Gold Coast, Australia; Sunil Badve, Department of Nephrology, St George Hospital, Sydney, New South Wales, Australia; Anish Scaria, George Institute of Global Health, Sydney, Australia; Stephane Heritier, Epidemiology and Preventative Medicine, Monash University, Melbourne, Australia.

Conflict of Interest Disclosures: Drs Irish and Hawley, Mss Pascoe and Paul-Brent, and Drs Mori, Cass, Kerr, and Polkinghorne report having received grant support from the National Health and Medical Research Council (NHMRC) project grant, grants from Mylan EPD (at the time of funding was Abbott Products Operations AG), and grants from Amgen Australia Pty Ltd. Dr Viecelli reports having received grant support from the National Health and Medical Research Council (Medical Postgraduate Scholarship) and the Royal Australasian College of Physicians (Jacquot National Health and Medical Research Council Medical Award for Excellence). No other disclosures are reported.

Funding/Support: The FAVOURED trial was funded by grants from National Health and Medical Research Council of Australia Project Grant (APP458652), Amgen Australia Pty Ltd and Mylan EPD (at the time of funding was Abbott Products Operations AG). Study medication was supplied by Mylan EPD (at the time of supply was Abbott Products Operations AG) (fish oil and placebo) and Bayer Healthcare (aspirin and placebo) free of charge.

Role of the Funder/Sponsor: The funders had no role in the design and conduct of the study; collection, management, analysis, and interpretation of the data; preparation, review, or approval of the manuscript; and decision to submit the manuscript for publication.

Previous Presentation: The findings of this trial were presented at the annual meeting of the American Society of Nephrology; November 7 , 2015; San Diego, California.

Additional Contributions: The authors listed in the byline constitute the FAVOURED Trial Writing Committee. The authors gratefully acknowledge the contributions of all members of the FAVOURED Study Collaborative Group, dialysis nursing staff, trial coordinators, research staff, and most especially trial participants.

\section{REFERENCES}

1. Manns B, Tonelli M, Yilmaz S, et al. Establishment and maintenance of vascular access in incident hemodialysis patients: a prospective cost analysis. J Am Soc Nephrol. 2005;16(1):201-209.

2. Polkinghorne KR, McDonald SP, Atkins RC, Kerr PG. Vascular access and all-cause mortality: a propensity score analysis. J Am Soc Nephrol. 2004:15(2):477-486.

3. Tordoir J, Canaud B, Haage P, et al. EBPG on vascular access. Nephrol Dial Transplant. 2007;22 (suppl 2):ii88-ii117.

4. Vascular Access Work Group. Clinical practice guidelines for vascular access. Am J Kidney Dis. 2006:48(suppl 1):S248-S273. 
5. Jindal K, Chan CT, Deziel C, et al; Canadian Society of Nephrology Committee for Clinical Practice Guidelines. Hemodialysis clinical practice guidelines for the Canadian Society of Nephrology. J Am Soc Nephrol. 2006;17(3)(suppl 1):S1-S27.

6. Polkinghorne KR, Chin GK, MacGinley RJ, et al. KHA-CARI Guideline: vascular access-central venous catheters, arteriovenous fistulae and arteriovenous grafts. Nephrology (Carlton). 2013;18 (11):701-705.

7. Ravani P, Palmer SC, Oliver MJ, et al. Associations between hemodialysis access type and clinical outcomes: a systematic review. J Am Soc Nephrol. 2013;24(3):465-473.

8. Ravani P, Gillespie BW, Quinn RR, et al. Temporal risk profile for infectious and noninfectious complications of hemodialysis access. J Am Soc Nephrol. 2013;24(10):1668-1677.

9. Lok CE, Sontrop JM, Tomlinson G, et al. Cumulative patency of contemporary fistulas versus grafts (2000-2010). Clin J Am Soc Nephrol. 2013;8(5):810-818.

10. Al-Jaishi AA, Oliver MJ, Thomas SM, et al Patency rates of the arteriovenous fistula for hemodialysis: a systematic review and meta-analysis. Am J Kidney Dis. 2014;63(3):464-478

11. Allon M, Robbin ML. Increasing arteriovenous fistulas in hemodialysis patients: problems and solutions. Kidney Int. 2002;62(4):1109-1124.

12. Palmer SC, Di Micco L, Razavian M, et al. Antiplatelet therapy to prevent hemodialysis vascular access failure: systematic review and meta-analysis. Am J Kidney Dis. 2013;61(1):112-122.

13. Dember LM, Beck GJ, Allon M, et al; Dialysis Access Consortium Study Group. Effect of clopidogrel on early failure of arteriovenous fistulas for hemodialysis: a randomized controlled trial. JAMA. 2008;299(18):2164-2171.

14. Mori TA, Beilin LJ, Burke V, Morris J, Ritchie J. Interactions between dietary fat, fish, and fish oils and their effects on platelet function in men at risk of cardiovascular disease. Arterioscler Thromb Vasc Biol. 1997:17(2):279-286.

15. Rylance PB, Gordge MP, Saynor R, Parsons V, Weston MJ. Fish oil modifies lipids and reduces platelet aggregability in haemodialysis patients. Nephron. 1986;43(3):196-202.

16. van Acker BA, Bilo HJ, Popp-Snijders $C$, van Bronswijk H, Oe PL, Donker AJ. The effect of fish oil on lipid profile and viscosity of erythrocyte suspensions in CAPD patients. Nephrol Dial Transplant. 1987;2(6):557-561.

17. Cartwright IJ, Pockley AG, Galloway JH, Greaves $M$, Preston FE. The effects of dietary omega-3 polyunsaturated fatty acids on erythrocyte membrane phospholipids, erythrocyte deformability and blood viscosity in healthy volunteers. Atherosclerosis. 1985;55(3):267-281.
18. Wang $Q$, Liang $X$, Wang $L$, et al. Effect of omega-3 fatty acids supplementation on endothelial function: a meta-analysis of randomized controlled trials. Atherosclerosis. 2012;221(2):536-543.

19. Fox PL, DiCorleto PE. Fish oils inhibit endothelial cell production of platelet-derived growth factor-like protein. Science. 1988;241 (4864):453-456.

20. Endres S, Ghorbani R, Kelley VE, et al. The effect of dietary supplementation with $n-3$ polyunsaturated fatty acids on the synthesis of interleukin-1 and tumor necrosis factor by mononuclear cells. N Engl J Med. 1989;320(5) 265-271.

21. Mori TA. Omega-3 fatty acids and cardiovascular disease: epidemiology and effects on cardiometabolic risk factors. Food Funct. 2014;5 (9):2004-2019.

22. Lok CE, Moist L, Hemmelgarn BR, et al; Fish Oi Inhibition of Stenosis in Hemodialysis Grafts (FISH) Study Group. Effect of fish oil supplementation on graft patency and cardiovascular events among patients with new synthetic arteriovenous hemodialysis grafts: a randomized controlled trial. JAMA. 2012;307(17):1809-1816.

23. Schmitz PG, McCloud LK, Reikes ST, Leonard $\mathrm{CL}$, Gellens ME. Prophylaxis of hemodialysis graft thrombosis with fish oil: double-blind, randomized, prospective trial. J Am Soc Nephrol. 2002;13(1): 184-190.

24. Baigent $C$, Blackwell $L$, Collins $R$, et al; Antithrombotic Trialists' (ATT) Collaboration. Aspirin in the primary and secondary prevention of vascular disease: collaborative meta-analysis of individual participant data from randomised trials. Lancet. 2009;373(9678):1849-1860.

25. Baigent C, Landray M, Leaper C, et al. First United Kingdom Heart and Renal Protection (UK-HARP-I) study: biochemical efficacy and safety of simvastatin and safety of low-dose aspirin in chronic kidney disease. Am J Kidney Dis. 2005;45 (3):473-484.

26. Irish A, Dogra G, Mori T, et al. Preventing AVF thrombosis: the rationale and design of the Omega-3 Fatty Acids (Fish Oils) and Aspirin in Vascular Access Outcomes in Renal Disease (FAVOURED) study. BMC Nephrol. 2009;10:1.

27. Viecelli AK, Pascoe EM, Polkinghorne KR, et al; FAVOURED Study Team. Baseline characteristics of the Omega-3 Fatty Acids (Fish Oils) and Aspirin in Vascular Access Outcomes in Renal Disease (FAVOURED) study. Nephrology (Carlton). 2016;21 (3):217-228.

28. Viecelli AK, Pascoe E, Polkinghorne KR, et al; FAVOURED study team. The Omega-3 Fatty Acids (Fish Oils) and Aspirin in Vascular Access Outcomes in Renal Disease (FAVOURED) study: the updated final trial protocol and rationale of post-initiation trial modifications. BMC Nephrol. 2015;16:89.

29. McNutt LA, Wu C, Xue X, Hafner JP. Estimating the relative risk in cohort studies and clinical trials of common outcomes. Am J Epidemiol. 2003;157 (10):940-943.

30. Bowden RG, Wilson RL, Gentile M, Ounpraseuth S, Moore P, Leutholtz BC. Effects of omega-3 fatty acid supplementation on vascular access thrombosis in polytetrafluorethylene grafts. J Ren Nutr. 2007;17(2):126-131.

31. Mori TA, Burke V, Puddey I, et al. The effects of [omega] 3 fatty acids and coenzyme Q10 on blood pressure and heart rate in chronic kidney disease: a randomized controlled trial. J Hypertens. 2009;27 (9):1863-1872.

32. Dixon BS. Why don't fistulas mature? Kidney Int 2006;70(8):1413-1422.

33. Lee T, Mokrzycki M, Moist L, Maya I, Vazquez M, Lok CE; North American Vascular Access Consortium. Standardized definitions for hemodialysis vascular access. Semin Dial. 2011;24 (5):515-524.

34. Smith GE, Gohil R, Chetter IC. Factors affecting the patency of arteriovenous fistulas for dialysis access. J Vasc Surg. 2012;55(3):849-855.

35. Lok CE, Allon M, Moist L, Oliver MJ, Shah H, Zimmerman D. Risk equation determining unsuccessful cannulation events and failure to maturation in arteriovenous fistulas (REDUCE FTM I). J Am Soc Nephrol. 2006;17(11):3204-3212.

36. Andrassy $\mathrm{K}$, Malluche $\mathrm{H}$, Bornefeld $\mathrm{H}$, et al. Prevention of p.o. clotting of av. cimino fistulae with acetylsalicyl acid: results of a prospective double blind study. Klin Wochenschr. 1974;52(7): 348-349.

37. Ferring M, Claridge M, Smith SA, Wilmink T. Routine preoperative vascular ultrasound improves patency and use of arteriovenous fistulas for hemodialysis: a randomized trial. Clin J Am Soc Nephrol. 2010;5(12):2236-2244.

38. Peterson WJ, Barker J, Allon M. Disparities in fistula maturation persist despite preoperative vascular mapping. Clin J Am Soc Nephrol. 2008;3 (2):437-441.

39. Manns B, Hemmelgarn B, Lillie E, et al. Setting research priorities for patients on or nearing dialysis. Clin J Am Soc Nephrol. 2014;9(10): 1813-1821.

40. Al-Balas A, Lee T, Young CJ, Barker-Finkel J Allon M. Predictors of initiation for predialysis arteriovenous fistula [published online September 14, 2016]. Clin J Am Soc Nephrol. 2016; doi:10.2215 /CJN.00700116. 
A randomised, double-blind, placebo-controlled, factorial-design trial to assess the effect of aspirin and fish oil (omega-3 fatty acids) in the prevention of early thrombosis in arterio-venous fistulae in patients with Stage IV or V chronic kidney disease requiring haemodialysis

FAVOURED (Fish oil and Aspirin in Vascular access OUtcomes in REnal Disease)

Trial Protocol

Version 8.1, 7 January 2010.

Principal Investigator

Dr Ashley Irish

Renal Unit, Royal Perth Hospital, Perth

phone: 61892242546

Ashley.Irish@health.wa.gov.au

Trial Management Committee

$\begin{array}{ll}\text { Dr Alan Cass } & \text { The George Institute for International Health, Sydney, Australia } \\ \text { Dr Vlado Perkovic } & \text { The George Institute for International Health, Sydney, Australia } \\ \text { Dr Sharan Dogra } & \text { Sir Charles Gardiner Hospital, Perth, Australia } \\ \text { Elaine Beller } & \text { University of Queensland, Brisbane, Australia } \\ \text { Ass Prof Carmel Hawley } & \text { Princess Alexandra Hospital, Brisbane, Australia } \\ \text { Professor Peter Kerr } & \text { Monash Medical Centre, Melbourne, Australia } \\ \text { Dr Trevor Mori } & \text { University of Western Australia, Perth, Australia } \\ \text { Dr Kevan Polkinghorne } & \text { Monash Medical Centre, Melbourne, Australia } \\ \text { Dr Amanda Robertson } & \text { Royal Melbourne Hospital, Melbourne, Australia } \\ \text { Dr David Voss } & \text { Middlemore Hospital, Auckland, New Zealand } \\ \text { Elvie Haluszkiewicz } & \text { Royal Perth Hospital, Perth, Australia } \\ \text { Dr David Gracey } & \text { Royal Prince Alfred Hospital, Sydney, Australia } \\ \text { Dr Amanda Mather } & \text { Royal North Shore Hospital, Sydney, Australia } \\ \text { Dr Stephen McDonald } & \text { Royal Adelaide Hospital, Adelaide, Australia } \\ \text { Dr Hooi Lai Seong } & \text { Hospital Sultanah Aminah, Johor Bahru, Malaysia } \\ \text { Dr Colin Hutchison } & \text { Queen Elizabeth Hospital, Birmingham, United Kingdom } \\ \text { Dr Chris Mclntyre } & \text { Derby City Hospital, Derby, United Kingdome } \\ \text { Peta-Anne Paul-Brent } & \text { University of Queensland, Brisbane, Australia } \\ \text { Trial Statistician } & \end{array}$

AKTN Project Officer:

Peta-Anne Paul-Brent

phone 61732405817

fax 61732405663

University of Queensland, School of Medicine

Ground Level, Building 33, Princess Alexandra Hospital

Ipswich Rd, Woolloongabba Queensland 4102 


\section{Table of contents}

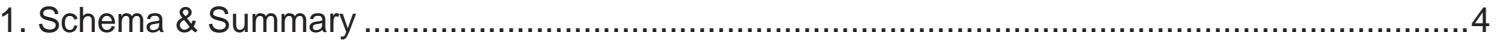

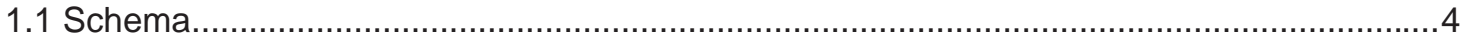

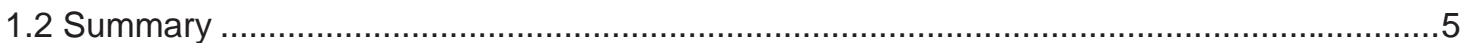

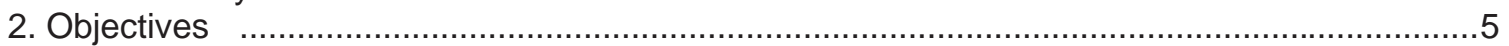

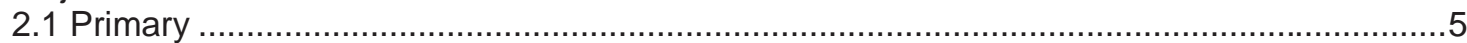

2.2 Secondary

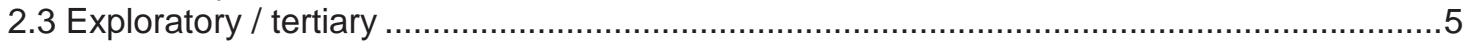

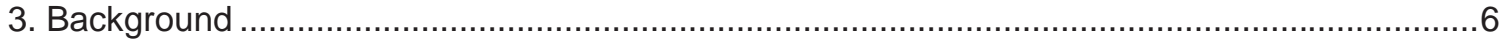

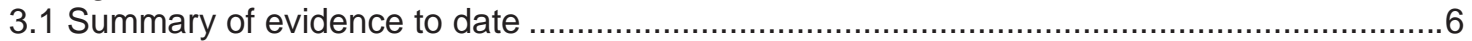

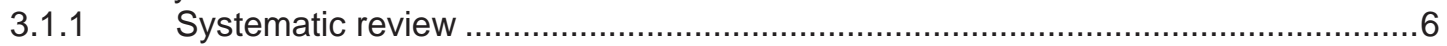

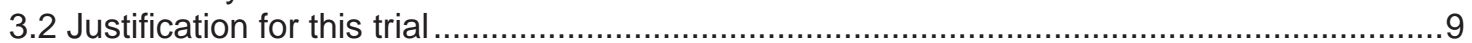

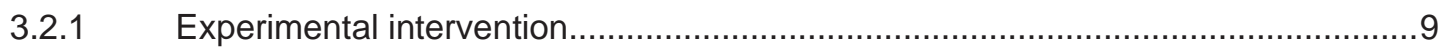

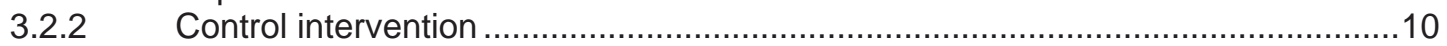

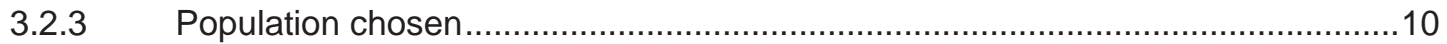

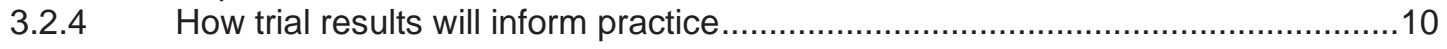

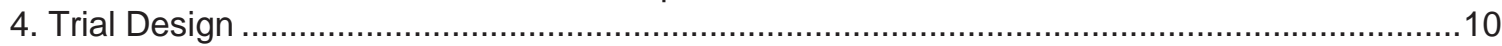

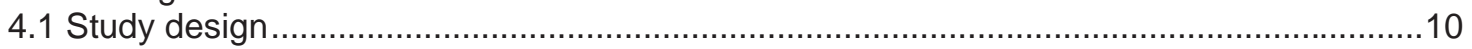

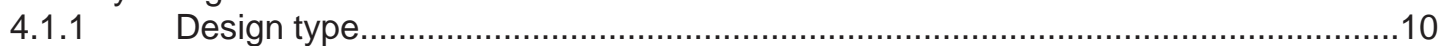

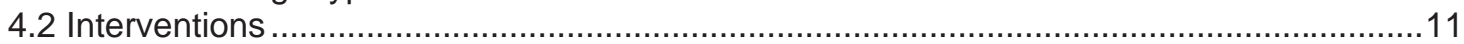

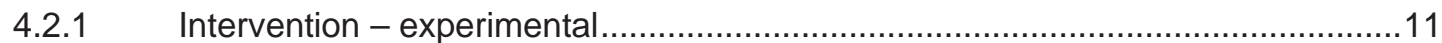

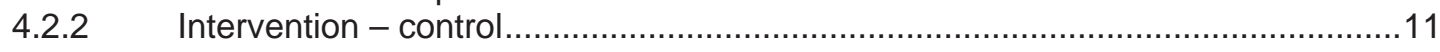

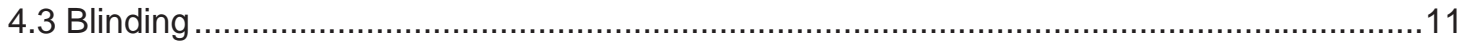

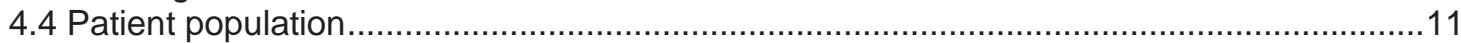

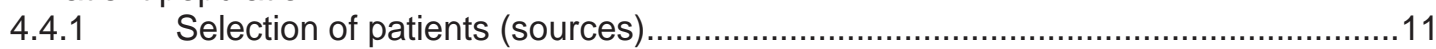

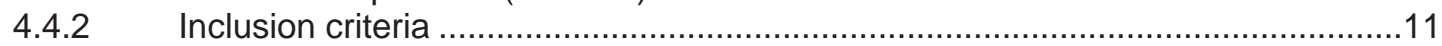

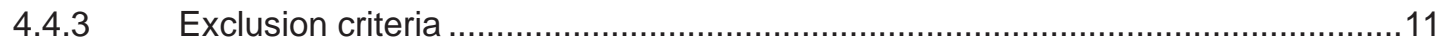

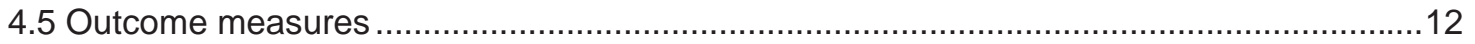

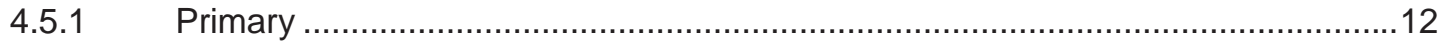

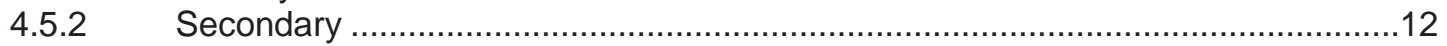

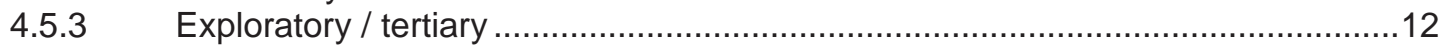

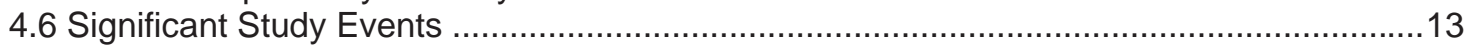

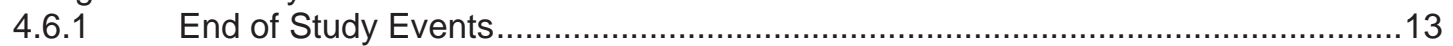

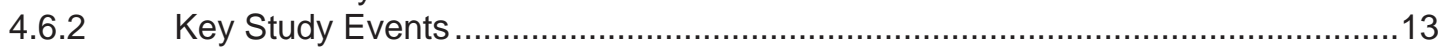

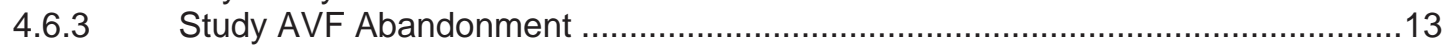

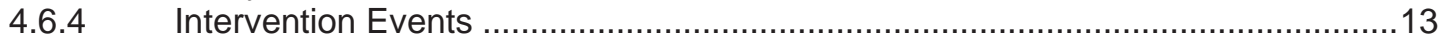

4.6.5 Patient withdrawal from study medication ....................................................... 14

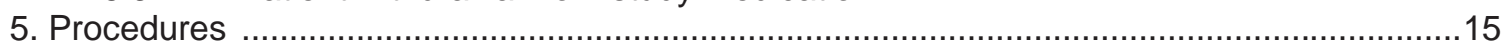

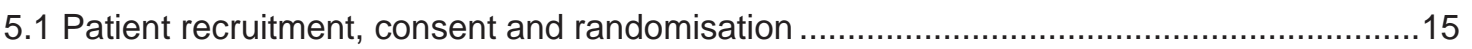

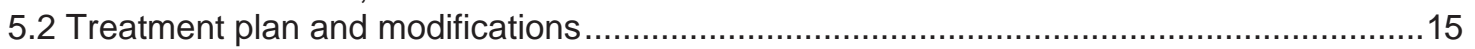

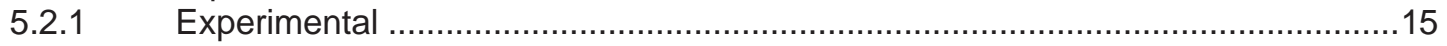

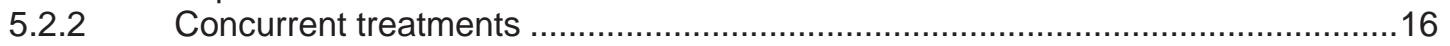

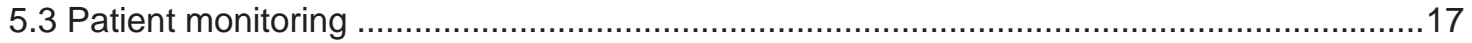

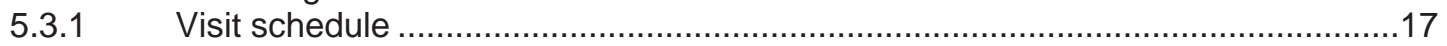

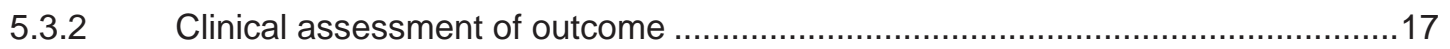

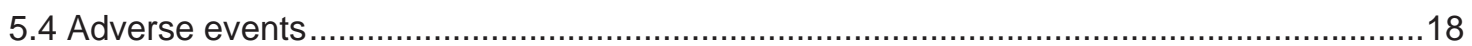

5.4.1 Reporting to Regulatory Authorities ................................................................19

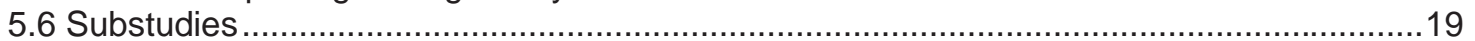

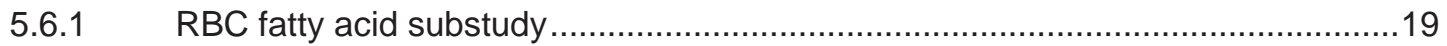

5.6.2 Dietary and physical questionnaire ............................................................. 19

5.6.3 Pre-surgical Doppler studies as a predictor of access outcome ..........................20

AKTN Protocol - Favoured Study

Version 8.1 07/01/2010 


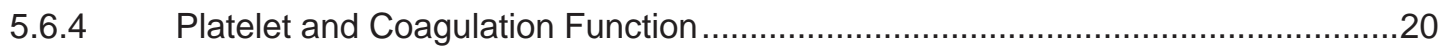

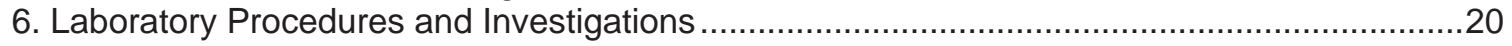

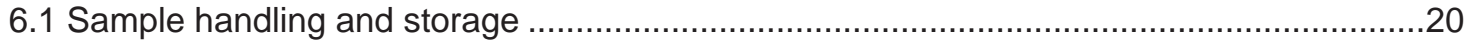

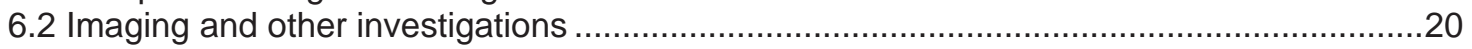

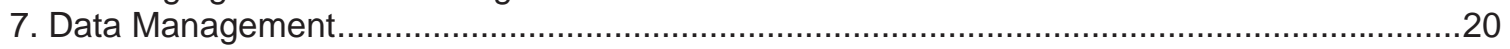

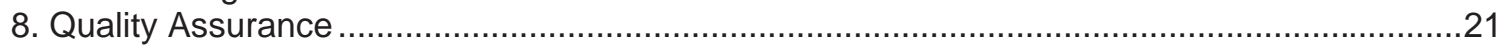

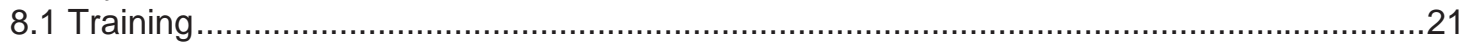

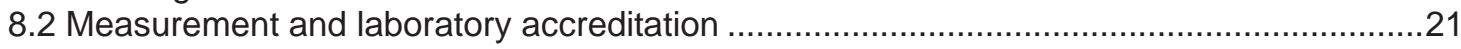

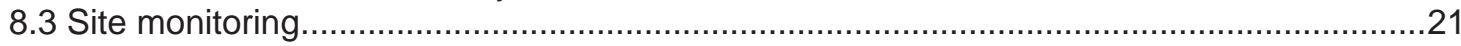

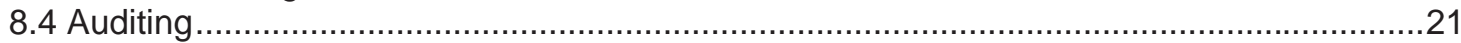

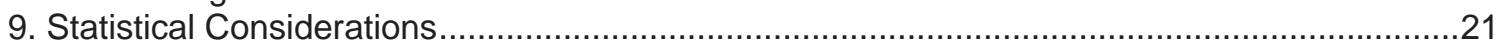

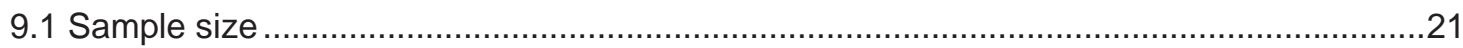

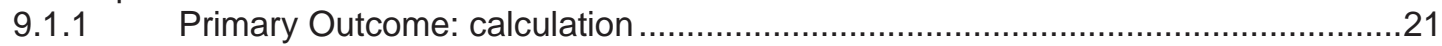

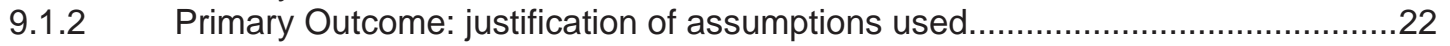

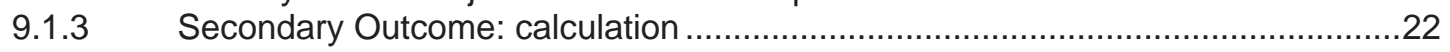

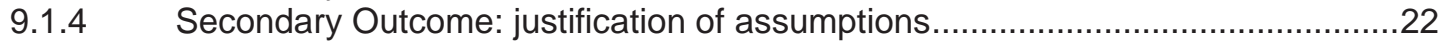

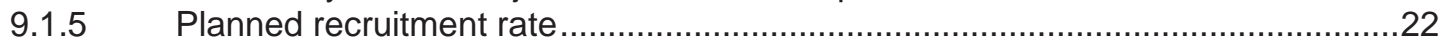

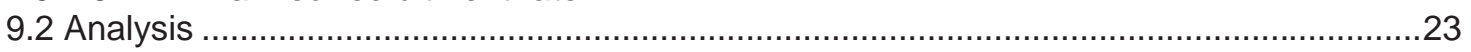

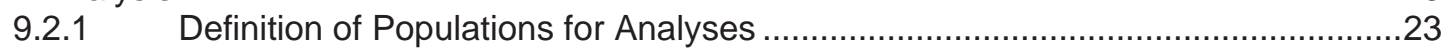

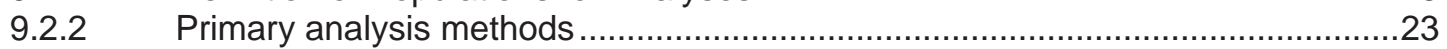

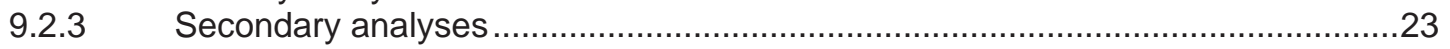

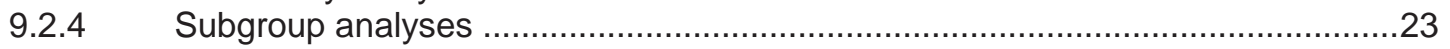

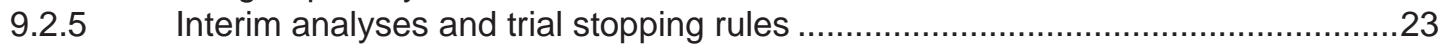

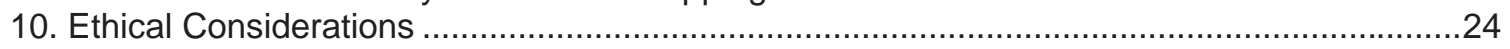

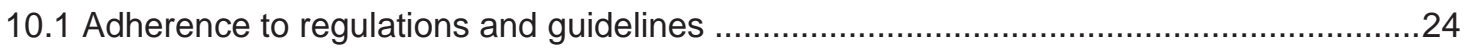

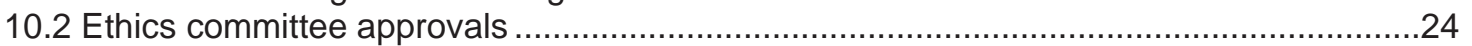

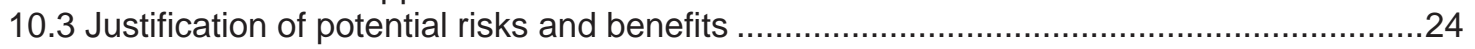

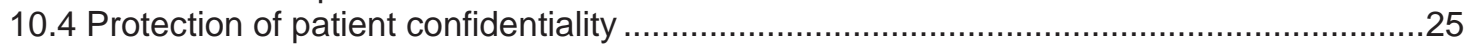

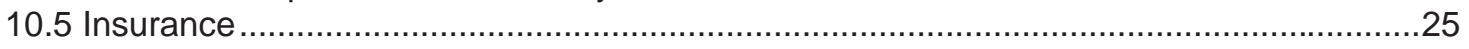

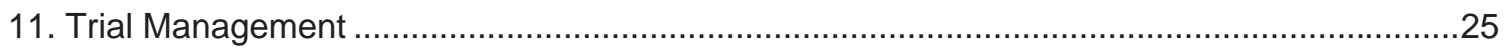

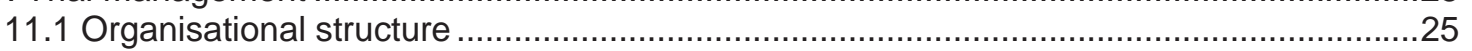

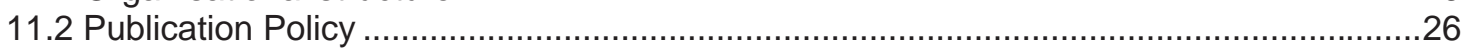

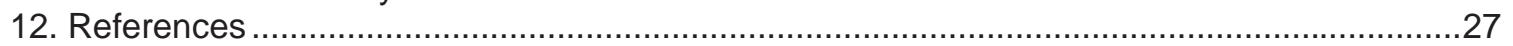

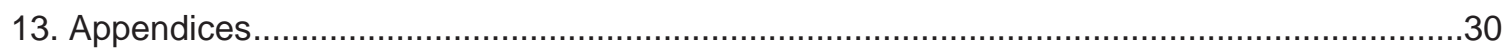

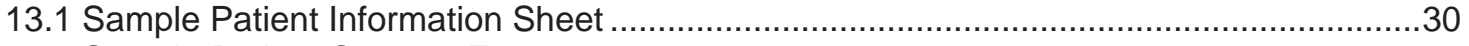

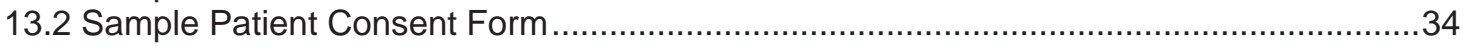

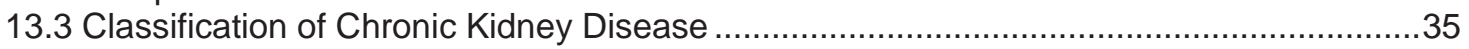

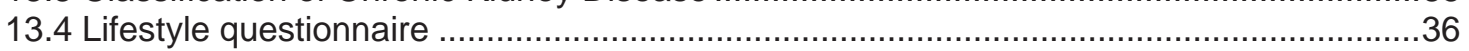

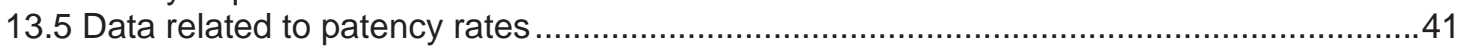

AKTN Protocol - Favoured Study

Version 8.1 07/01/2010 


\section{Schema \& Summary}

\subsection{Schema}

Patient Population
Inclusion:
- $\quad$ Stage 4 or 5 CKD patients undergoing or planning to
undergo HD with planned de novo AVF
Exclusion:
- AVF revision.
- $\quad$ Medical indication for anti-platelet agents or known
intolerance of agents.

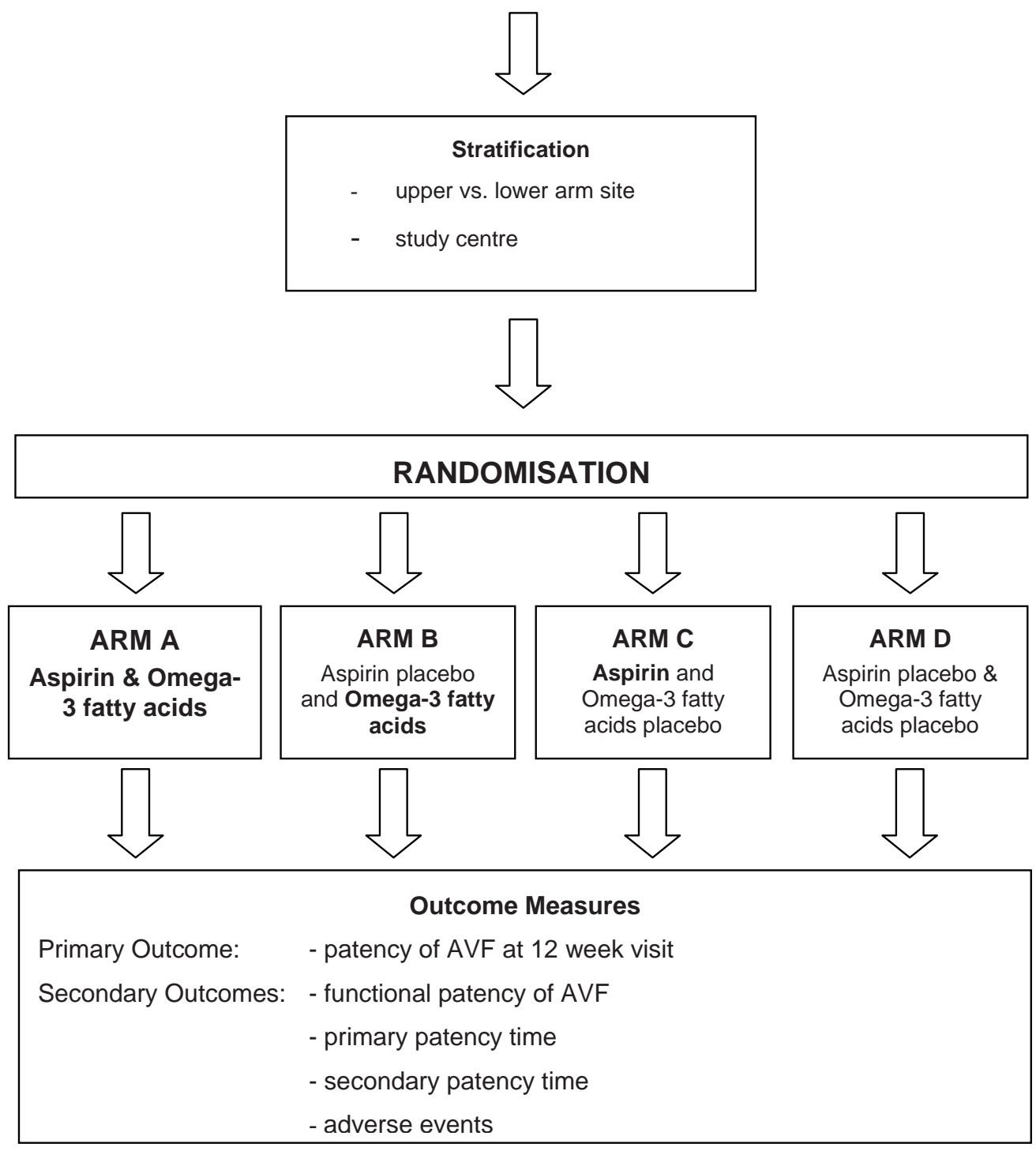

AKTN Protocol - Favoured Study

Version 8.1 07/01/2010 


\subsection{Summary}

This study aims to determine whether the use of the anti-platelet agents aspirin and omega-3 fatty acids, either alone or in combination (in a factorial design), will effectively reduce the risk of early thrombosis in de novo arterio-venous fistulae (AVF). The study population is patients with stage IV or V chronic kidney disease (CKD) who require or will require haemodialysis, who are scheduled to undergo creation of an AVF and who are not currently taking anti-platelet agents. The primary outcome is patency of the AVF at three months after surgery. Other outcomes include functional patency at various times during the study, primary patency time, secondary (assisted) patency time, and adverse events, particularly bleeding.

For the purposes of this protocol "omega-3 fatty acids" refers to the two marine-derived long-chain polyunsaturated fatty acids, eicosapentaenoic acid (EPA) and docosahexaenoic acid (DHA).

\section{Objectives}

\subsection{Primary}

To determine whether aspirin and/or omega-3 fatty acids effectively reduces the risk of early thrombosis in arterio-venous fistulae, when compared with placebo and where the outcome is defined as patency at three months after surgery.

\subsection{Secondary}

1. To determine whether aspirin and/or omega-3 fatty acids effectively reduces the risk of early thrombosis in arterio-venous fistulae, compared with placebo, where the secondary outcome is defined as functional patency at six months after surgery.

2. To determine whether aspirin and/or omega-3 fatty acids effectively reduces the risk or early thrombosis in arterio-venous fistulae compared with placebo, where the secondary outcome is defined as functional patency at twelve months after surgery.

3. To determine whether aspirin and/or omega-3 fatty acids effectively reduces the risk of early thrombosis in arterio-venous fistulae, compared with placebo, where the secondary outcome is defined as functional patency at any point after surgery.

4. To determine whether aspirin and/or omega-3 fatty acids effectively increases the time from AVF creation to the time of first therapeutic intervention to maintain patency, compared with placebo (primary patency time).

5. To determine whether aspirin and/or omega-3 fatty acids effectively increases the time from AVF creation until final access abandonment, compared with placebo (secondary patency time).

6. To determine the adverse event rates for aspirin and/or omega-3 fatty acids, particularly bleeding events, compared with placebo.

\subsection{Exploratory / tertiary}

1. To determine whether aspirin and/or omega-3 fatty acids effectively reduces the risk of thrombosis in arterio-venous fistulae in the immediate post surgery period where the outcome is defined as patency in the first 24 hours after surgery. 
2. To determine whether aspirin and/or omega-3 fatty acids effectively reduces the risk of thrombosis in arterio-venous fistulae at 6 weeks after surgery, where the outcome is defined as patency at 6 weeks after surgery.

3. To determine whether aspirin and/or omega-3 fatty acids effectively reduces the time to first cannulation of the AVF ie time taken from the time of surgery until the first successful attempt at access cannulation.

4. To determine whether aspirin and/or omega-3 fatty acids effectively reduces the time to consistent cannulation of the AVF ie time taken is from the time from surgery until that of the 3rd consecutive dialysis session with successful cannulation of the access.

\section{Background}

\subsection{Summary of evidence to date}

\subsubsection{Systematic review}

Severe chronic kidney disease (CKD) is increasing in both incident and prevalent cases due to ageing of the population and co-morbid conditions such as diabetes. Haemodialysis is utilised by $70 \%$ of patients with end-stage kidney disease (ESKD) in Australia. However, effective haemodialysis is critically dependent upon obtaining and maintaining repeated access to the circulation. Delivering the required blood flow $(>300 \mathrm{ml} /$ minute) necessary for haemodialysis has long been referred to as the "Achilles' heel" of dialysis. Vascular access options include native arterio-venous fistula (AVF), synthetic arterio-venous graft (AVG) or central venous catheter (CVC). AVF is universally acknowledged as the optimal access device with the best long-term patency, lowest cost and lowest infection rate [1-3] and is the most prevalent access used in Australasia (75\%)[4]. Because vascular access related surgical procedures and complications of vascular access represent a high proportion of all admissions in the ESKD population and are a major economic burden for health care providers[5], various international initiatives have made this a focus of quality care improvements [6].

Specifically the complication rates associated with artificial vascular access devices (AVG and CVC) are sepsis, especially staphylococcal, vascular malfunction (thrombosis) and death. The increased risk of death is directly attributable to sepsis and the need for interventions to restore patency, which by their invasive nature, enhance the risk of infection. Several studies confirm the risk (Hazard Ratio) of all cause death is (using AVF as the reference group) around 1.5 for AVG and 3 for CVC and similar rates for infectious mortality $[2,3,7]$. CVC and AVG have a significantly increased risk, frequency and costs of interventions to maintain patency (assisted patency) than native AVFs.

Therefore, while the clinical imperative is to establish AVF in as many patients as possible, primary failure because of early thrombosis and failure of maturation are the major impediments to clinical success [8]. Reports in the literature from the 1970s and 1980 s generally describe primary failure rates of between $10-20 \%$. More contemporary publications (1990's on) are less encouraging with reports of the primary failure rate increasing to between $20-54 \%$. The higher rate of primary failure in the more recent era may reflect changing patient factors - such as older patients and a higher prevalence of diabetes. A meta-analysis examining the primary failure rate of AVF has been done [9]. In this study the pooled primary failure rate was estimated to be $15.3 \%$. However, this meta-analysis included studies back to the 1960s and omitted a number of studies published after the meta-analysis. These more recent studies have a higher primary failure rate (20-54\% in studies published since 1999)[1]. Consistent with these findings of 
a high early failure rate, $50 \%$ of new patients commence haemodialysis in Australia and New Zealand with a CVC, due to a failed or inadequate AVF [10].

Primary failure usually occurs as a result of one of two processes [8]:

1) Thrombosis, which usually occurs within weeks of the procedure;

2) Inadequate size of the artery or maturation of the vein.

Strategies to try and reduce primary failure rates may therefore include pre-operative identification of unsuitable anatomy by the use of ultrasound, improved surgical technique and pharmacological interventions designed to prevent vessel occlusion (thrombosis).

Early thrombosis is defined as thrombosis within the first 30 days post-operatively [11]. There have been a number of small trials evaluating pharmacological agents aimed at reducing the early thrombosis rate. These trials utilised the anti-platelet agents aspirin, sulphinpyrazone and ticlopidine and varied in size from as few as 5 patients up to 261 patients. Andrassey et al $(n=92)$ compared patients given aspirin $500 \mathrm{mg} /$ day for 4 weeks with placebo: the thrombosis rate in the aspirin group was $4 \%$ compared with the control group of $24 \%$, OR $0.15(0.03,0.73)$ [12]. Another study [13] with a smaller sample size $(n=68)$, did not demonstrate a benefit from aspirin. The aspirin studies were mostly in grafts, not fistulae, and the mechanism of thrombosis is believed to differ. Fistula studies have been very small, utilising a wide range of agents and doses, and done mostly in the 1970s. Therefore this study is ethically justifiable. Our survey of Aust/NZ sites indicates few patients are routinely given aspirin. In this study, where patients receive aspirin for non-access related conditions then clinicians are to use clinical discretion regarding the safety of withdrawal, (recognising that the no RCT has established clinical benefit for aspirin in kidney failure patients).

In a study using ticlopidine[14], fistula thrombosis rates for the ticlopidine versus placebo groups were $12 \%$ versus $19 \%(p=0.10)$ respectively. Studies using sulphinpyrazone showed variable results, but were underpowered with the largest study enrolling only 36 patients [15]. Pooled data for ticlopidine suggests a reduction in thrombosis rate from $25 \%$ to $12 \%(p<0.001)$ [14]. Thus although there is some data to suggest that antiplatelet agents may increase the primary patency of AVF, the limited evidence base and the uncertainty regarding the choice of agent has not supported the widespread use of anti-platelet agents in the prevention of AVF thrombosis. There is a strong clinical need to conduct an adequately powered clinical trial to address the important question of efficacy of anti-platelet therapy in the prevention of early thrombosis of AVF.

One study has examined the effects of low-dose aspirin in combination with omega-3 fatty acids on whole blood eicosanoid production [16]. This did demonstrate an additive effect of the combination on TX2 (40\% with aspirin alone vs. $62 \%$ with the combination) and a smaller reduction in the concentrations of PGI2 and PGI3 compared with aspirin alone. There has only been one study exploring the efficacy of omega-3 fatty acids in the prevention of vascular access thrombosis. This study involved the use of omega-3 fatty acids as a single agent and only explored its use in AVG, not AVF. Importantly, the study demonstrated a dramatic reduction in thrombosis at 1 year in patients with AVG [17]. There are other effects of omega-3 fatty acids, which may be particularly beneficial in patients with CKD. Omega-3 fatty acids have been shown to result in an improvement in lipid profile[17-19], BP and heart rate reduction [20], and attenuated inflammatory responses [21] and oxidative stress[22]. Other postulated but as yet unproven benefits in the CKD population include a reduction in inflammatory response, cardiovascular mortality and a reduction in uraemic pruritis [23].

AKTN Protocol - Favoured Study

Version 8.1 07/01/2010

Page 7 of 43 
It is an excellent time to study the effects of anti-platelet agents in the prevention of AVF thrombosis. The number of patients requiring dialysis is growing and the maintenance of vascular access in these patients is one of the major challenges faced by health workers in this area, both in terms of clinical input required and economic burden. Furthermore, studies on the natural history of vascular access outcomes show an increase in the incidence of primary failure, which is most likely related to the changing demographics of patients with an increasing prevalence of older patients and diabetic status. Moreover, there has never been an adequately powered study examining the efficacy of antiplatelet agents in the prevention of AVF. AVF is the most commonly utilised vascular access in Australia and New Zealand and thus an excellent setting to conduct this study.

A recently published placebo-controlled parallel arm study studied the prevention of early thrombosis in AVFs using Clopidogrel (an antiplatelet agent) with study treatment commencing one day post surgery with a primary outcome of patency at 6 weeks [24]. There was a $12.2 \%$ thrombosis rate in the Clopidogrel arm vs. $19.5 \%$ in the control arm: RR of $0.63(0.46-0.97) p=0.018$, adjusted for interim analyses. Suitability of the AVF for dialysis failed in $63 \%$ of the clopidogrel group and in $60 \%$ of the placebo group $(61 \%$ overall). There were no differences in adverse events and, surprisingly, bleeding events were very low in both trial arms. The conclusions drawn by the investigators were that although Clopidogrel is effective in safely preventing primary AVF thrombosis, it was ineffective at increasing functional patency and therefore cannot be recommended.

The implications this trial for the Favoured Study include the following:

1. The high functional failure rate is especially concerning and much higher than expected. This may reflect aspects related to surgical (e.g. small number of procedures performed by individual surgeons) and nursing care (needling skills for AVF) in the US and/or patients demographics. Furthermore, significant variation between USA and both European and Australian clinical practice could explain the high US study failure and unsuitability rates. In this context, the generalisability of this American study to ANZ is uncertain and justifies additional trial confirmation in other countries;

2. Earlier administration of the active agents before surgery (as in Favoured) rather than after may lead to an even greater efficacy in relation to the primary thrombosis rate;

3. The rational for trialing two agents in a factorial design remains valid. In fact, fish oil help may be of additional benefit by improving vascular endothelial function and smooth muscle relaxation, which may enhance maturation and greater attainment of functional patency;

4. The low bleeding rates and safety data with Clopidogrel are reassuring in the context of uncertainty regarding the safety of anti-platelet therapy in renal failure, given that anecdotally Clopidogrel is considered to be associated with a higher risk of bleeding than aspirin;

5. The greater duration of follow up (minimum of 1 year) in the Favoured Study will allow greater ascertainment of the secondary outcome measure with relation to functional patency. This is almost more essential in light of the Clopidogrel Study result.

These studies do not negate the need for a trial examining the place of a cheaper, less risk associated therapy such as aspirin or omega-3 fatty acids, should either prove beneficial.

AKTN Protocol - Favoured Study

Version 8.1 07/01/2010

Page 8 of 43 


\subsection{Justification for this trial}

The failure of vascular access is the most common cause for hospital admission in CKD patients.

\subsubsection{Experimental intervention}

Aspirin has been chosen as one of the interventions in this study because of its wellestablished anti-platelet effects, the trend to efficacy in the underpowered studies to date and because it is both inexpensive and an agent familiar to clinicians. Its use as an antiplatelet agent is well established in clinical practice for other purposes relating to thrombosis prevention particularly in patients with established cardiovascular disease. The anti-platelet effect of aspirin is mediated by its ability to inhibit the platelet cyclooxygenase enzyme resulting in blockade of the synthesis of the pro-aggregatory vasoconstrictor, thromboxane A2 (TxA2).

Omega-3 fatty acids have a number of biological effects which make it an attractive agent for the prevention of vascular access thrombosis, including the inhibition of platelet aggregation, anti-inflammatory effects and anti-proliferative actions. Omega-3 fatty acids and aspirin both affect the balance between the pro-aggregatory and vasoconstrictor effects of TXA2 and the anti-aggregatory and vasodilator effects of prostacyclin (PGI2) but the mechanism of their effect is different. Omega-3 fatty acids have a weaker inhibitory effect on TxA2 level, its effect mediated by reducing the availability of arachidonic acid (AA), a precursor of TxA2. In addition, omega-3 fatty acids lead to an increase in PGI3 formation (anti-aggregatory and vasodilatation effects equipotent to PGI2) [16]. The anti-inflammatory effect of omega-3 fatty acids is mediated via a reduction in leukotriene and cytokine production. Theoretically then, a combination of aspirin and omega- 3 fatty acids should result in a more favourable effect on platelet aggregation than either agent used alone.

We have chosen an oral route of administration for both aspirin and omega-3 fatty acids in this study. The choice of $100 \mathrm{mg}$ for aspirin is based on this dose having adequate antiplatelet aggregation properties and is the dose most commonly used in patients with cardiovascular disease. A $100 \mathrm{mg}$ dose is readily available. The dose of omega-3 fatty acids ( $4 \mathrm{~g}$ daily) has been shown in our hands to be well tolerated and has benefited multiple cardiovascular risk factors in patients at increased risk of cardiovascular disease [25-29]. The omega-3 fatty acid capsules chosen (Omacor@), are commercially available and provide the highest concentration of omega-3 fatty acids per gram of oil. The oral absorption of both agents is excellent and thus alternative routes of administration are not required.

Bleeding episodes are a concern for clinicians in relation to the use of aspirin in this cohort. Even in the general population, on chronic low dose aspirin the risk of serious gastrointestinal bleeding is doubled [30] and the theoretical risk is higher in patients with CKD because of the presence of impaired haemostasis from multiple mechanisms. There have been published studies [31, 32], showing a significant elevation in bleeding times in patients on haemodialysis treatments administered a single dose of aspirin, but surprisingly little evidence-based clinical trial data in this population. This has more recently been explored in the UK-HARP-I study. In this factorial design study involving simvastatin and aspirin as active therapies, allocation to the aspirin group was not associated with an excess of major bleeds ( $2 \%$ in patients on aspirin vs. $3 \%$ in patients not on aspirin), but there was a three-fold increase in minor bleeding [33]. Importantly 
though, in this study, patients received aspirin for 12 months, whereas our study has a much shorter period on therapy of 3 months. Exploring the potential risk of even shortterm aspirin is a major secondary end-point of this study.

Omega-3 fatty acids appear to be well tolerated, even in high doses, with gastrointestinal complaints particularly nausea, vomiting, diarrhoea and non-specific discomfort being the most often reported. In studies looking at the effect of omega-3 fatty acids on bleeding time it has not been found to be significantly prolonged [34]. In studies that have been done thus far in the CKD cohort, there have been no clear effects demonstrated in relation to platelet aggregation and bleeding times. There has only been one report of a serious bleeding event in a single patient in an uncontrolled study [35]. Furthermore, it has been reported that administration of omega- 3 fatty acids can protect the gastric mucosa against aspirin-induced injury, the postulated mechanism being that omega- 3 fatty acids counteract the effect of aspirin on the decrease in prostacyclin (combined effect of PGI2 and PGI3) [36, 37]. Thus the combination of aspirin and omega-3 fatty acids may be expected to be additive in terms of efficacy in vascular access thrombosis, but to have a lower risk of bleeding complications than the use of aspirin alone.

The design of a factorial study is based on the hypothesis that aspirin and omega- 3 fatty acids will both be effective therapies and that the combination of aspirin and omega- 3 fatty acids will be additive, but not synergistic. In relation to safety concerns, although both aspirin and omega- 3 fatty acids prolong bleeding time, the combination may be expected to be safer than aspirin used alone.

\subsubsection{Control intervention}

Currently patients are not given any prophylactic agents in the majority of cases. This is because the studies to date have not been definitive in demonstrating an effect and concern exists about the safety of the agents in respect to bleeding events. Therefore use of placebo is appropriate.

\subsubsection{Population chosen}

The study will be multi-centre, and open to all public and private hospital renal units that perform vascular access procedures and will be conducted in Australia and selected overseas countries such as New Zealand, the United Kingdom, China and Malaysia.

\subsubsection{How trial results will inform practice}

If the trial demonstrates a positive effect of either or both agents, this will lead to a reduction in thromboses, quicker time to dialysis access, and a need for less revisional surgery.

If the trial demonstrates no effect of the agents, this will suggest that platelet aggregation is not the major mechanism for thrombosis in this population and that it may be the poor status of vessels used to create the anastomosis that is a more important factor.

\section{Trial Design}

\subsection{Study design}

\subsubsection{Design type}

This is a factorial-design trial, where patients are randomised to aspirin or matching placebo, and also to omega-3 fatty acids or matching placebo, resulting in four treatment groups. Randomisation will be achieved using a dynamic balancing method, balancing over the two stratification factors of 1) study site and 2) upper versus lower arm AVF. 


\subsection{Interventions}

\subsubsection{Intervention - experimental}

The principal action of both aspirin and omega- 3 fatty acids is by their ability to reduce platelet aggregation. Omega-3 fatty acids have other potentially beneficial effects on rheology, inflammation and blood flow.

\subsubsection{Intervention - control}

Matching placebo for each agent.

\subsection{Blinding}

Each of the two interventions will be studied using a matching placebo, in order to make this a double-blind trial. At the completion of treatment, patients and investigators will be asked which treatment they believe the patient has been receiving, in order to have a measure of the degree of effectiveness of blinding.

In addition, the primary outcome of patency assessment will be made by an independent observer, unaware of the patient's treatment assignment and treatment course or medical history.

\subsection{Patient population}

\subsubsection{Selection of patients (sources)}

Patients will be recruited through renal units. The study will be multi-centre, and conducted in Australia and selected overseas countries such as New Zealand, the United Kingdom, China and Malaysia. Public hospital renal unit haemodialysis patients are likely to represent the full population of haemodialysis patients well, as most such patients are treated in these units.

\subsubsection{Inclusion criteria}

Patients must meet the following inclusion criteria:

1. Stage 4 or 5 chronic kidney disease

2. Currently on haemodialysis or haemodialysis is planned to start within 12 months (including patients currently on peritoneal dialysis).

3. Planned AVF will be the primary haemodialysis access mechanism.

4. Surgery to create an arterio-venous fistula in the upper or lower arm is planned.

5. Aged over 19 years

6. Treating team agreeable to patient's involvement in the trial

7. Informed consent

\subsubsection{Exclusion criteria}

Patients must not meet any of the following exclusion criteria:

1. Revision of existing AVF rather than de novo AVF

2. Medical indication for anti-platelet or thrombolytic agents

3. Known intolerance of agents including hypersensitivity to aspirin, allergy to any other NSAIDs or fish

4. Current use of aspirin within two weeks of commencing trial, or of omega-3 fatty acids within 4 weeks of commencing trial.

5. Pregnancy, lactation or intention to fall pregnant during the time course of the study

6. Known bleeding disorder or established diagnosis of active or suspected bleeding

7. History of Gl ulcers or bleeding within the last 3 months

8. Platelet count less than $100 \times 10^{9} / \mathrm{L}$ 
9. Known active peptic ulcer disease

10. Severe hepatic insufficiency

11. Already receiving anti-coagulation therapy such as warfarin

12. Receiving regular non-steroidal anti-inflammatory (NSAIDS) agents for another indication such as arthritis

13. Syndrome of asthma, rhinitis and nasal polyps if uncontrolled on usual therapy

14. Plan to have other (non-access) surgery within 2 weeks of trial medication period where, in the opinion of the investigator, aspirin or fish oil would be contraindicated for the planned procedure.

15. Potential non-compliance with treatment regimen in the view of the treating clinicians

16. Involved in another clinical trial where the intervention being trialled is likely to confound the outcome of this trial

17. Previously randomised to this trial.

\subsection{Outcome measures}

\subsubsection{Primary}

The primary outcome measure is (unassisted) patency of the AVF at 12 week study visit. This is defined as the presence of an audible bruit over the site of the arterio-venous anastomosis for patients who have not experienced an intervention event or AVF abandonment. Please refer to section 4.6.4 for details of the intervention events. The assessment of patency will be made at this time even if the AVF is not yet used for haemodialysis. Measurement of bruit will also be taken at 1 week to better define the natural history of thrombotic events.

\subsubsection{Secondary}

1. Functional patency at 6 months. This is defined as whether the study AVF has been successfully cannulated at a minimum of 3 consecutive haemodialysis treatments and has since been in continuous use until the time of the 6 month study visit.

2. Functional patency at 12 months. This is defined as for the outcome measure functional patency at 6 month but is intended to capture the results of patients whose commencement of dialysis is delayed beyond 6 months.

3. Functional Patency at any time. This is defined as the ability to use the study AVF for a minimum of 3 consecutive haemodialysis treatments at any time point during the study.

4. Time to failure of primary patency. Time from AVF creation to first intervention event or AVF abandonment whichever occurs first. Please refer to section 4.6.4 for details of the intervention events. Censored observations are defined as recovery of renal function, renal transplant, patient lost to follow up, patient withdrawal from study, death or still having a patent AVF at time of study analysis. There will be a minimum of 12 months follow up time for each patient.

5. Time to failure of assisted patency. This is defined as time from AVF creation to abandonment of access. That is, radiological and surgical interventions to preserve or restore patency do not constitute failure. Censored observations will be as for time to failure of primary patency.

6. Adverse events. All serious adverse events and adverse drug reactions (see section 5.4) will be collected. The analysis of this secondary outcome will focus specifically on bleeding events.

\subsubsection{Exploratory / tertiary}

1. Patency in the first 24 hours post surgery. This is determined by the presence or absence of a bruit over the site of the anastomosis, with standard stethoscope. 
2. Patency at 6 weeks post surgery. This is determined by the presence or absence of a bruit over the site of the anastomosis.

3. Time to first successful cannulation of the AVF. This is the time taken from the time of surgery until the first successful attempt at access cannulation. Censored observations will be as for time to failure of primary patency.

4. Time to consistent cannulation of the AVF. This is time taken from the time from surgery until that of the 3rd consecutive dialysis session with successful cannulation of the access. Censored observations will be as for time to failure of primary patency.

\subsection{Significant Study Events}

\subsubsection{End of Study Events}

End of Study Events are events that mean the end of study with no further follow up with participants. End of study events include death, withdrawal of patient's consent, patient is lost to follow up and the end of study (12 months from the last patient's surgery).

The ANZDATA registry patient information may be accessed to confirm end of study events, particularly for patients lost to follow up.

\subsubsection{Key Study Events}

Key Study Events are events that do not mean the end of study for patients but are important to record with relation to study outcomes measures and the patient progress in the study. These events include:

- Recovery of renal function (such that dialysis is not longer needed).

- Renal transplant.

- Thrombosis of the study AVF.

- Imaging of the study AVF.

\subsubsection{Study AVF Abandonment}

Abandonment of the study AVF is defined as the point that which the AVF is unable to be cannulated and no further attempts will be made to rescue or revise the access.

The date that the AVF is abandoned is determined at the discretion of site PI. However, events that may indicate AVF abandonment include ligation of study AVF, thrombosis in study AVF with no intension to revise, imaging of study AVF that shows that the AVF is unusable and the insertion of replacement dialysis access including new AVF, AVG, venous catheter or peritoneal dialysis access.

\subsubsection{Intervention Events}

There are two types of the interventions events, interventions that cause end of primary patency and interventions that do not cause end of primary patency (but should be recorded).

Interventions to the study AVF that mean the end of primary patency include:

- Medical or surgical thrombectomy.

- Surgical or radiological revision of anastomosis or dilation of vein/artery within $8 \mathrm{~cm}$ of the anastomosis eg angioplasty etc.

Interventions to the Study AVF that do not cause end of primary patency include:

- Dilation of central venous stenosis.

- Radiological or surgical dilatation of vein/artery further than $8 \mathrm{~cm}$ from Anastomosis.

- Ligation of tributaries.

- Superficialisation of AVF. 
- Ligation of fistula or salvage by DRIL (Distal reconstruction and interval Ligation) due to distal ischaemia (steal).

\subsubsection{Patient withdrawal from study medication}

Withdrawal of study medication may be made at the discretion of the investigator following a serious adverse event or key study events. However, subjects should still be followed for the duration of the study if at all possible, even if they have ceased treatment. This will include attendance at scheduled trial visits, and data collection, particularly of outcomes. The investigator may withdraw a subject from study medication at any time if it is felt to be in the best interest of the subject.

Study treatment may be discontinued if one of the following occurs:

- A major bleeding event (e.g. haemorrhagic stroke, gastro-intestinal bleed).

- The access is abandoned due to thrombosis at any time after the one week assessment.

- An AVG rather than an AVF is created, or a vein graft is used.

- Anti platelet therapy or anticoagulation is indicated because of other co morbid events i.e. major cardiovascular event.

- A female patient becomes pregnant while on study medication. The event should be reported to the trial coordinating centre immediately as a medically important SAE. The event will be reported within 24 hours and follow up performed through to delivery.

- The AV anastomosis is created in the lower limbs rather the upper limbs during surgery.

- Patient receives renal transplant.

Study treatment should be continued if:

- Further access is created (including a new AVF or AVG, peritoneal and venous catheter) because of failure of maturation of the study AVF, i.e. access is still patent.

- Renal function is recovered; the study AVF can still be measured patency.

Table 2: Study medication continuation based on key study event or SAE occurrence

\begin{tabular}{|c|c|}
\hline Study Outcomes & $\begin{array}{c}\text { Continue } \\
\text { Medication? }\end{array}$ \\
\hline Renal transplant & No \\
\hline Renal function recovered & Yes* \\
\hline Thrombosis of study AVF & $\begin{array}{c}\text { No } \\
\text { if occurs after 1 week }\end{array}$ \\
\hline Pregnancy & No \\
\hline New AVF or AVG & Yes $^{*}$ \\
\hline Peritoneal dialysis & Yes $^{*}$ \\
\hline commenced & Yes $^{*}$ \\
\hline Venous catheter & No \\
\hline Major bleed & No \\
\hline Study AVG created & No \\
\hline Anti-platelet therapy & No \\
\hline Lower limb access & \\
\hline${ }^{*}$ at the treating physicians discretion &
\end{tabular}


Patients withdrawn from treatment will still be regarded as being "on study", and follow up data will be collected, unless the patient withdraws consent. No other anti-platelet or anticoagulation agent is permitted during the period the patient is taking study medication.

If a patient removes consent for the study they shall be withdrawn from the study. Any patient is free to remove their consent at any time without the need to justify their decision.

\section{Procedures}

\subsection{Patient recruitment, consent and randomisation}

Participants will be recruited from renal units providing haemodialysis and vascular access services in Australia and selected overseas countries such as New Zealand, the United Kingdom, China and Malaysia. Patients will meet the inclusion and exclusion criteria and be scheduled to undergo surgery for creation of a de novo AV fistula.

Patient consent forms will be approved by the Human Research Ethics Committee at each participating site prior to the beginning of the trial. A sample consent form and patient information sheet is provided to participating sites. Participating sites will file a copy of the approved consent form and information sheet for their site with the coordinating office. After discussing the trial, ample time will be given to the participant, accompanying person or legal representative to inquire about the trial and decide whether to participate. No person involved with the trial will coerce or unduly influence the decision of a patient to participate in a trial. A copy of the signed consent form and the patient information sheet will be supplied to the participant. Patient consent must be obtained prior to the registration or initiation of trial procedures. Patients will not be randomised until a signed consent form is filed at site

The Trial Management Committee will monitor the medical literature, and any other relevant information impacting on the continuation of the trial. Consent forms and patient information sheets will be revised should any relevant and important new information become available.

Patients are to be randomised as close as possible to the time of the scheduled procedure and not more than 7 days before the planned procedure. Patients are to commence taking study medications on the day prior to the scheduled surgery. If the procedure is rescheduled, the patient is to cease taking medications if already started and to recommence the day before the rescheduled surgery. Refer to the operations manual for further information. Stratification will occur for study site and upper vs. lower arm site. Patients will be randomised to one of four treatment groups in equal proportion.

\subsection{Treatment plan and modifications}

\subsubsection{Experimental}

Aspirin $100 \mathrm{mg}$ per day p.o. or matching placebo, supplied by Bayer Healthcare, commencing on the day prior to scheduled surgery and continuing for 3 months.

Omega-3 fatty acids $4 \mathrm{gm}$ daily in the form of 4 Omacor capsules (46\% eicosapentaenoic acid (EPA) and 38\% docosapentaenoic acid (DHA), as the ethyl esters), or 4 matching placebo capsules (olive oil), supplied by Solvay Pharmaceuticals Australia, commencing on the day prior to surgery and continuing for 3 months.

To improve compliance and minimise gastro-intestinal discomfort the omega-3 fatty acids or matching placebo medications are to be taken morning and evening, 2 capsules taken 
with a cold drink just before breakfast and 2 capsules again taken with a cold drink just before dinner.

If patient's access surgery is postponed, patient should stop medication until $24 \mathrm{hrs}$ before rescheduled surgery. If the surgery is postponed for more than 4 weeks, the patient must have another blood test and their eligibility (conformity with the inclusion/exclusion criteria) must be reassessed. If they remain eligible, they should commence the medication which they have already been allocated.

Compliance will be monitored by capsule/tablet count at scheduled study visits at 6 and 12 weeks. Centres will also collect erythrocyte fatty acid samples for testing for compliance with omega-3 fatty acid intake. Centres will apply routine biochemistry (lipids, $\mathrm{CRP}$ ) and haematology analyses as part of patient monitoring (refer to section 6.1)

\subsubsection{Concurrent treatments}

No other fish oil or omega-3 fatty acid supplement, anti-platelet or anti-coagulation agents is permitted during the period the patient is taking study medication. Heparin or heparin-like agents that are used during vascular surgery or during haemodialysis to flush lines are permitted during the study treatment period.

In addition, interaction of the following agents with aspirin should be considered:

- Thrombolytic agents

- Methotrexate, particularly at doses of $15 \mathrm{mg} /$ week or greater

- Uricosuric agents

- Digoxin

- Sufonylureas or insulin

- Systemic glucocorticoids, except replacement therapy for Addison's disease

- ACE inhibitors, beta blockers

- Valproic acid, phenytoin

- Acetazolamide

- Diuretics (if daily asa dose is $>3 g$ ) 


\subsection{Patient monitoring \\ 5.3.1 Visit schedule}

Table 2: Favoured Study Visit Schedule

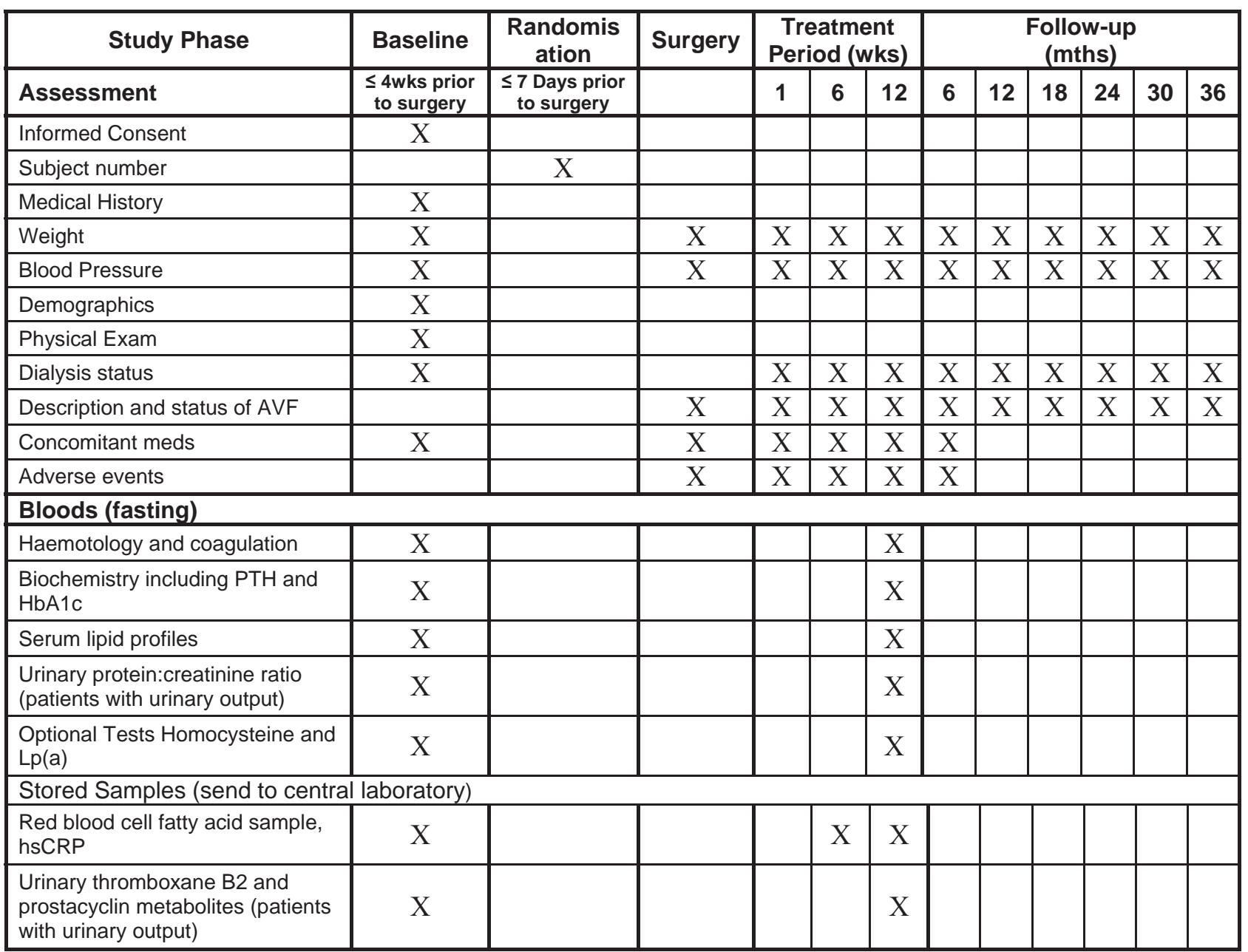

Notes:

- Baseline visit is to be completed within 4 weeks prior to surgery. It is recommended that all baseline procedures occur at the same visit but consent and physical exam for example can occur on a separate day.Patients are to be randomised as close as possible to the time of the scheduled procedure and not more than 7 days before the planned procedure. Patients are to commence taking study medications on the day prior to the scheduled surgery. If the procedure is rescheduled, the patient is to cease taking medications if already started and to recommence the day before the rescheduled surgery.

\subsubsection{Clinical assessment of outcome}

Two separate observers will independently record the presence or absence of bruit at site of anastomosis. A standard stethoscope will be used (not a Doppler stethoscope). 
The bruit must be present over the site of anastomosis. In the event that the two observers disagree on the presence of the bruit, the bruit would be recorded as absent.

Bruit will be assessed by medical or nursing staff, specifically

- Consultant nephrologists

- Advanced trainees

- Access surgeons

- Dialysis nurses

- Vascular access nurses

In addition, bruit assessment at discharge post surgery may be performed by recovery nurses.

\subsection{Adverse events}

Adverse events, including any cases of bleeding, will be managed as per usual local clinical care practice.

Adverse Events and Serious Adverse Events will be reported during study medication period and 3 months afterwards only. Only AEs that the site investigator rates as possibly or probably related with the study medication will be recorded. These will primarily include bleeding events and gastrointestinal symptoms. All SAEs will be recorded, whether related to the study medication or not. AEs and SAEs will be recorded in the regular data collection activities of the trial.

For the purposes of this trial the following definitions will apply;

Adverse Event:

An adverse event is defined as "any untoward medical occurrence in a patient or clinical investigation subject administered a pharmaceutical product which does not necessarily have a causal relationship with this treatment"

Serious Adverse Event:

A serious adverse event (experience) or reaction is any untoward medical occurrence that at any dose:

- results in death

- is life threatening (refers to an event in which the patient was at risk of death at the time of the event)

- requires inpatient hospitalisation or prolongation of existing hospitalisation

- results in persistent or significant disability/ incapacity

- is a congenital anomaly/ birth defect or

- is a medically important event or reaction.

Medical and scientific judgment should be exercised in deciding whether other situations should be considered serious such as important medical events that might not be immediately life-threatening or result in death or hospitalisation but might jeopardise the patient or might require intervention to prevent one of the other outcomes list in the definitions above.

Suspected Unexpected Serious Adverse Reaction (SUSAR)

Any adverse reaction that is both serious and suspected to be caused by the study medication that is not consistent with the information in the applicable product information (unexpected) is subject to expedited reporting to the TGA.

Adverse events will be recorded in the regular data collection activities of the trial.

AKTN Protocol - Favoured Study

Version 8.1 07/01/2010

Page 18 of 43 
If the $\mathrm{AE}$ is assessed as serious, the site PI must report the event to the Coordinating Office immediately or within $\mathbf{2 4}$ hours of being made aware of the event. The initial report should be made using the SAE Fax Notification sheet and an accompanying report that includes at a minimum: the name \& contact details of the reporter, the event \& seriousness criteria met, the patient's ID number \& treatment pack number and date the reporter became aware of the event.

If required, a more detailed follow-up report is to be submitted, within 7 days of the initial report submission, to the Coordinating Office and to the institutional ethics committee (if this is required by that committee). Please refer to local ethics committee guidelines as they may have a specific form they require for SAE reports

The follow up written report includes: the patient's identification number, a description, seriousness and likely cause of the SAE, concomitant medications, duration of the SAE with start and end dates, and details of reporter

A 24 hour contact number will operate to allow site staff to contact an appropriate representative of the trial management committee if they believe breaking the blind is warranted due to adverse events. The representative will discuss and if necessary negotiate the necessity of breaking the blind. If this action is deemed necessary, the time, date and reason will be recorded.

\subsubsection{Reporting to Regulatory Authorities}

The responsibility for reporting serious, unexpected and associated adverse drug events to the Drug Safety Evaluation Board of the Therapeutic Goods Administration lies with the study coordinating office. The study coordinating office is also responsible for reporting all serious adverse events to Solvay Pharmaceuticals and Bayer Drug Safety as per their contractual agreements.

\subsection{Substudies}

\subsubsection{RBC fatty acid substudy}

Omega-3 fatty acids incorporated into the red blood cell membrane will be used as a compliance measure. In addition, there will be a cross sectional assessment looking at RBC omega-3s and patient characteristics.

RBC fatty acids will be measured for all patients before the commencement of study medications (at baseline), once the patient has been taking the study medications for 6 weeks and as close as possible to the last dose of study medications. 2-3 ml volume samples will be collected into EDTA, labelled with study number, patient initials and date of collection and stored frozen at -20 degrees or colder. Samples will be sent to the central testing laboratory in batches as advised. Complete details of collection, storage and shipping requirements are provided in the study operations manual.

\subsubsection{Dietary and physical questionnaire}

It is well known that CKD patients have poor diets and malnutrition. This sub-study explores the status and the significance of dietary intake (including fish) and lifestyle factors in relation to patient outcomes. In particular, the questionnaire results will enable the interaction between these lifestyle and dietary factors and response to fish oil supplementation to be explored

Trial participants will be asked to complete the dietary and physical questionnaire (appendix 13.4) once only, at baseline. Questionnaires will ideally be completed whilst the patient is in clinic and reviewed by the research nurse/clinical trial coordinator while 
the patient is still on site and any missing information completed at that time. Original responses should be kept in the patient's trial folder.

\subsubsection{Pre-surgical Doppler studies as a predictor of access outcome}

This sub-study explores whether pre-operative vascular characteristics determined by duplex ultrasound will predict vascular access outcomes either independently or in particular, the response to aspirin, fish oil and the combination.

Interested centres with vascular laboratories will perform standardised evaluation and reporting of arm vasculature by duplex ultrasound prior to surgery. The outline of the standardised methods for evaluation will be provided in the study operations manual.

\subsubsection{Platelet and Coagulation Function}

This substudy aims to study the effects of aspirin and fish oil alone and the combination on platelet function, coagulation and platelet activation as well as quantification of compliance with aspirin (prostaglandin metabolites).

At baseline and week 12 spot urine samples will be collected from participants with urinary output for the measurement of protein:creatinine ratio (to be tested by the local laboratory) and urinary thromboxane B2 and prostacyclin metabolites (a $5 \mathrm{~mL}$ sample stored at $20^{\circ} \mathrm{C}$ or less, to be sent with the RBC fatty acid blood samples for testing by the central laboratory).

\section{Laboratory Procedures and Investigations}

\subsection{Sample handling and storage}

Apart from samples for the RBC fatty acid substudy, urinary thromboxane B2 and prostacyclin metabolites and measurement of hsCRP, all other routine blood and urine samples will be analysed by local laboratories, and samples will be collected in accordance with the requirements of these laboratories. The required sampling points for the routine blood tests are shown in table 5.3.1. For the blood samples taken at baseline and Week 12 patients must fast as per individual sites requirements for fasted lipid and glucose tests. Samples for the RBC fatty acid and platelet function substudies will be collected and stored as per section 5.6.1. The routine blood tests required are:

1) Haematology and Coagulation: Full Blood Count (including $\mathrm{Hb}$, WCC and platelets) and coagulation (INR, APTT and fibrinogen).

2) Biochemistry: Creatinine, urea, electrolytes, calcium and phosphate, albumin, fasting glucose, parathormone and $\mathrm{HbA} 1 \mathrm{c}$. The protein:creatinine ratio of patients with urine output will be tested. The $\mathrm{HbA} 1 \mathrm{c}$ and parathormone levels measured within 4 weeks prior to randomisation are acceptable as baseline measurement.

3) Fasting Lipids: Total, LDL, HDL and triglycerides.

4) Optional tests (where these are routinely available) are fasting Homocysteine and Lipoprotein(a).

\subsection{Imaging and other investigations}

Imaging and other investigations will be performed as per normal local practice.

\section{Data Management}

AKTN Protocol - Favoured Study

Version 8.1 07/01/2010

Page 20 of 43 
The timing of forms to be completed follows the schedule in Section 5.3.1 and is outlined in more detail in the operations manual.

Original consent forms are to be stored locally.

All source documents used in this trial, including medical records, should be stored for a period of 15 years following the close of the study.

\section{Quality Assurance}

\subsection{Training}

Assessment of bruit is to be performed according to guidelines provided.

\subsection{Measurement and laboratory accreditation}

A copy of the NATA or other accreditation certificate and local normal ranges will be sent to the coordinating centre prior to the use of any local laboratories.

\subsection{Site monitoring}

The coordinating centre reserves the right to visit sites for study monitoring purposes. At these visits, the authorised study monitor will require access to the medical records of study patients for the purpose of source data verification. The study monitor will also have access to all other study documentation, including ethics correspondence for the purpose of verifying appropriate study management.

\subsection{Auditing}

The coordinating centre reserves the right to audit sites. At these visits, the authorised study auditor will require access to the medical records of study patients.

\section{Statistical Considerations}

\subsection{Sample size}

\subsubsection{Primary Outcome: calculation}

The event rate for the primary outcome (failure of primary patency) is estimated to be $25 \%$ at three months in the control group. If a $30 \%$ relative risk reduction is achievable with either aspirin or omega-3 fatty acids, then with $80 \%$ power and a significance level of $5 \%, 1200$ study subjects will be required (300 receiving double placebo, 300 active aspirin and placebo omega-3 fatty acids, 300 active omega-3 fatty acids and placebo aspirin, and 300 both active aspirin and active omega- 3 fatty acids in a factorial design). This allows for $5 \%$ drop-in from placebo to active treatment (either aspirin or omega-3 fatty acids), $5 \%$ drop-out from study treatment (to no treatment - assumed equivalent to placebo), and $5 \%$ loss to follow-up. This is equivalent to an observed relative risk reduction of $24 \%$. Without adjustment for compliance, the study size needed would have been 932 subjects.

The study is not adequately powered to detect a clinically important difference between the combination of aspirin and omega-3 fatty acids and either treatment alone, but preliminary data on the combination will be obtained. 


\subsubsection{Primary Outcome: justification of assumptions used}

The event rate of the primary outcome, primary failure, is based on review of all papers published in this area. The effect size, a 30\% reduction, is based on the smallest size of the effect the proposing clinicians believe would lead to a change in practice (incorporation of intervention) should the trial prove the intervention is efficacious.

\subsubsection{Secondary Outcome: calculation}

Sample size is estimated for the secondary outcome (failure of patency at 6 months) under assumptions of additive or multiplicative effects for each of 3 placebo rates. The calculation below is based on the following assumptions: $5 \%$ loss to FU, $15 \%$ drop-in and $5 \%$ drop-out.

The baseline failure rate of patency at 6 months is (40\%, 50\% and $60 \%)$ in the placebo group, the use of either aspirin or omega- 3 fatty acids alone results in a relative risk reduction (RRR) of $25 \%$, i.e. the failure rate becomes respectively $30 \%, 37.5 \%$ and $45 \%$ for a patient taking only one drug.

The sample size calculation is based on a marginal analysis (Z-test) with two-sided $\alpha=$ 0.05 , power $\beta=90 \%$. Table below presents required sample sizes under additive model (grey) and under multiplicative model

\begin{tabular}{|c|c|c|c|c|}
\hline \multirow{3}{*}{$\begin{array}{l}\text { Patency failure rate at } 6 \\
\text { months for placebo group }\end{array}$} & \multicolumn{4}{|c|}{ Total sample size } \\
\hline & \multicolumn{2}{|c|}{ Additive model } & \multicolumn{2}{|c|}{ Multiplicative model } \\
\hline & Unadjusted & Adjusted & Unadjusted & Adjusted \\
\hline $40 \%$ & 874 & 1455 & 1156 & 1812 \\
\hline $50 \%$ & 620 & 1034 & 820 & 1297 \\
\hline $60 \%$ & 452 & 752 & 596 & 941 \\
\hline
\end{tabular}

\subsubsection{Secondary Outcome: justification of assumptions}

A comparable trial in the United States [24] experienced a placebo rate of $60 \%$ for failure of patency at 6 months. We are confident that a sample size based on a placebo rate of at least $50 \%$ is a realistic estimate and that the sample of 1,200 recruited for the primary outcome will generally be adequate for the secondary outcome whether the effect is additive or multiplicative.

\subsubsection{Planned recruitment rate}

Based on data from the ANZDATA renal registry [24, 38], 7,202 patients were receiving dialysis in Australia and New Zealand in December 2004. Importantly, 2042 patients commenced dialysis in Australia and New Zealand in the calendar year 2004. Data obtained from the whole of New Zealand population and access rates for Princess Alexandra Hospital (PAH), Queensland, reveal that 1 procedure is performed for every 2.3 - 2.8 patients receiving haemodialysis (total numbers of patients - new patients and prevalent patients). The New Zealand data was obtained by Dr. Mark Marshall (survey from October 12000 until September 30, 2001) and by Dr. Carmel Hawley (Queensland) based on prospectively collected data in PAH renal unit from January 2004 until December 2004. In addition further information from the 2005 registry report supports these data. Incident patients require access for dialysis and $27 \%$ and $33 \%$ of haemodialysis patients in Australia and New Zealand, respectively, were incident patients in 2004. Thus we can expect these patients at the very least to require new vascular access, in addition to existing patients on dialysis who need further access 
because of failure of previous vascular access procedures. Extrapolation to the whole Australian and New Zealand population, using a conservative estimate of 1 procedure per 3 patients allows us to derive that 2,400 access procedures are performed per year (AVF and AVG). Using a conservative estimate that $75 \%$ of access is AVF and not AVG, gives an estimation of 1,800 de-novo AVF creations per year. Assuming we will recruit $22 \%$ of these, recruitment will take approximately three years.

It is anticipated that the addition of sites from other countries will facilitate the recruitment target being reached within the projected time frame.

\subsection{Analysis}

\subsubsection{Definition of Populations for Analyses}

Analysis of the primary outcome (patency) will be on an intent-to-treat basis. All randomised patients will be analysed in the group they were allocated to, even if they do not receive treatment as allocated, or do not commence treatment. All randomised patients will be included in the analysis.

\subsubsection{Primary analysis methods}

This is a factorial design with the primary analysis being

a) aspirin vs. placebo (with and without omega-3 fatty acids) and

b) omega-3 fatty acids vs. placebo (with and without aspirin).

The primary outcome is measured as the proportion with failure of patency at three months after surgery. The primary hypothesis will be tested using a chi-square test of proportions in a marginal analysis. Specifically, we will test the null hypotheses that a) the proportion of failures in those allocated to aspirin versus those allocated to placebo aspirin is equal, and $b$ ) the proportion of failures in those allocated to omega- 3 fatty acids versus those allocated to placebo omega-3 fatty acids is equal, ignoring the other factor A more refined analysis based on logistic regression model that includes the two factors (with possibly an interaction) and the stratification variables (site and position of AVF) will also be carried out.

The primary outcome will be measured at 84 days after surgery, with a permitted tolerance of 7 days prior, and 14 days after the due date of 84 days.

Tests of hypotheses will be at the $5 \%$ significance level, and 2 -sided $p$-values will be used.

\subsubsection{Secondary analyses}

A secondary analysis will be done including only those with an 84 day measure to test the sensitivity of the analysis of the main treatment comparisons to patient excursions.

\subsubsection{Subgroup analyses}

Not applicable

\subsubsection{Interim analyses and trial stopping rules}

Safety reports will be produced regularly and sent to the Safety and Data Monitoring Committee (SDMC). Two formal interim analyses will be conducted after $1 / 3$ and 2/3 of total patients have been accrued and followed for sufficient time. The Haybittle-Peto rule will be used as stopping guideline for efficacy as it offers the flexibility to update the number of looks if need be. If two interim analyses equally-spaced are indeed performed, the level of the final analysis will be 0.048. Similar analyses to the primary one will be undertaken on the secondary outcomes, as well as exploratory analyses looking at the 
validity of the chosen primary outcomes and its correlation with other outcomes, and the prediction of thrombosis from baseline variables such as markers of inflammation. SAEs or AEs will also be formally examined by the DSMB. Arrangements will be made to provide the Chair of the SDMC and possibly other members with regular information on fatalities and life-threatening SAEs possibly related to trial treatment (e.g. intracranial haemorrhage).

\section{Ethical Considerations}

\subsection{Adherence to regulations and guidelines}

This trial will adhere to the regulations, guidelines and standards espoused in the World Medical Association Declaration of Helsinki, the NHMRC National Statement of Ethical Conduct in Research Involving Humans, the joint NHMRC/AVCC Statement and Guidelines on Research Practice, and the Therapeutic Goods Administration Note for Guidance on Good Clinical Practice.

\subsection{Ethics committee approvals}

Patients will not be enrolled until the final approved protocol, as well as any trial information for patients is reviewed and approved by a Human Research Ethics Committee.

Correspondence with HRECs should be copied and supplied to the coordinating centre. It is the responsibility of the investigators to ensure that the appropriate person from each site is aware of the reporting responsibilities of each site to the relevant HRECs.

\subsection{Justification of potential risks and benefits}

The potential benefits to the patients are those relating to a reduction in AVF thrombosis rate and the associated benefits of lower hospitalisation rate, lower risk of needing alternative vascular access procedures and further attempts at establishing permanent vascular access. The only significant concern in this study is that of bleeding complications. This risk relates primarily to the group assigned to aspirin alone and to the group assigned combined aspirin and omega-3 fatty acid therapy.

The relationship between aspirin use and an increased risk of bleeding is a potential concern in patients with CKD. In the general population, the use of a chronic low dose of aspirin doubles the risk of serious gastrointestinal bleeding [30] and the theoretical risk may be higher in patients with CKD because of the presence of uraemic induced impairment of haemostasis. There have been published studies[31, 32], showing a significant elevation in bleeding times in patients on haemodialysis treatments administered a single dose of aspirin, but surprisingly little evidence-based clinical trial data in this population. This has more recently been explored in the UK-HARP-I study. In this factorial design study involving simvastatin and aspirin as active therapies, allocation to the aspirin group was not associated with an excess of major bleeds $(2 \%$ in patients on aspirin vs. $3 \%$ in patients not on aspirin), but there was a three-fold increase in minor bleeding[33]. Importantly though, in this study, patients received aspirin for 12 months, whereas our study has a much shorter period on therapy of 3 months. In addition, patients with ESRD on dialysis undergoing renal transplantation have successfully 
received pre-operative aspirin for the prevention of graft thrombosis without an increased risk of major bleeding[39, 40].

Omega-3 fatty acids appear to be well tolerated, even in high doses, with gastrointestinal complaints particularly nausea, vomiting, diarrhoea and non-specific discomfort being the most often reported. In studies looking at the effect of omega- 3 fatty acids on bleeding time it has not been found to be significantly prolonged [34]. In studies that have been done thus far in the CKD cohort, there have been no clear effects demonstrated in relation to platelet aggregation and bleeding times. There has only been one report of a serious bleeding event in a single patient in an uncontrolled study [35]. Furthermore, it has been reported that administration of omega-3 fatty acids can protect the gastric mucosa against aspirin-induced injury, the postulated mechanism being that omega-3 fatty acids counteract the effect of aspirin on the decrease in prostacyclin (combined effect of PGI2 and PGI3)[36]. This is supported by clinical data suggesting that omega-3 fatty acids decrease gastric erosions and ulcers caused by aspirin or alcohol[37]. Thus the combination of aspirin and omega- 3 fatty acids may be expected to be additive in terms of efficacy in vascular access thrombosis, but to have a lower risk of bleeding complications than the use of aspirin alone.

It is important to perform this study to carefully explore the risks and benefits of aspirin and omega-3 fatty acids as single agents or as combination therapy.

\subsection{Protection of patient confidentiality}

Patients' records and the data generated by the study will be confidential in line with the recommendations of the NHMRC and the 2001 privacy legislation. Any information that may identify a patient will be excluded from data presented in the public arena. Data must be stored in a secure, lockable location. Electronic data storage must have adequate password protection. Standardised case report forms will be provided for each subject on this study. The participants in this study will be identified only by initials and subject number on these forms. De-identified information may only be released to the AKT Network or designee.

\subsection{Insurance}

Insurance will be provided through the University of Queensland.

\section{Trial Management}

\subsection{Organisational structure}

The Trial Management Committee (TMC) is comprised of persons appointed through AKTN procedures. Representatives include the trial proposer (chair), two members of the AKTN Scientific Committee, study biostatistician, a member of the AKTN Operations Secretariat, and others as required. It is the responsibility of this group to provide leadership to the overall conduct of the trial. In particular, this group will review the progress of the study in achieving the objectives, take appropriate decisions to meet these objectives, and make decisions regarding the continuation or modification of a trial given reports from the Safety and Data monitoring committee.

AKTN Protocol - Favoured Study

Version 8.1 07/01/2010

Page 25 of 43 
Specifically, the TMC will:

- Monitor the blinded event rate for comparison with the one used for design purposes

- Monitor missing data

- Forward to the SDMC, on an ongoing basis, details of treatment-emergent deaths and life-threatening SAEs possibly related to treatment

- Ensure that treatment withdrawals are continuously monitored

- Communicate any notable imbalance between treatment groups to the SDMC, including details on the reason for withdrawal

The TMC oversees the trial-related activities of the coordinating office. The TMC reports to the AKTN Scientific Committee.

The Safety and Data Monitoring Committee (SDMC) will be comprise of a statistician, an experienced clinical trialist and two Nephrologists who have no involvement with the day to day running of the study. The SDMC will be responsible for reviewing the interim analyses of safety (to be conducted at $1 / 3$ and $2 / 3$ of total patient numbers accrual), and will advise the Trial Management Committee when the stopping rules have reached or any other matters of safety that arise during the course of the study.

\subsection{Publication Policy}

The Australasian Kidney Trial Network subscribes to the criteria for authorship formulated by the International Committee of Medical Journal Editors (ref):

"Each author should have participated sufficiently in the work to take public responsibility for the content. Authorship credit should be based only on substantial contributions to

(a) conception and design, or analysis and interpretation of data; and to

(b) drafting the article or revising it critically for important intellectual content; and on

(c) final approval of the version to be published.

Conditions (a), (b), and (c) must all be met."

The main authorship will appear as follows:

The writing committee (listed: Chief PI listed first, then remaining members listed alphabetically) for the FAVOURED Study Collaborative Group.

On the front page, the following will be listed:

Members of the FAVOURED Study Collaborative Group are listed in the Supplementary Appendix, available with the full text of this article.

Authorship in the appendix will appear in the following order:

Writing Committee

Trial Management Committee

Steering Committee (the PI at each site)

Project Management Team (AKTN)

Safety and Data Monitoring Committee

Data Management/IT

All investigators and Study Coordinators at each site

The TMC will approve all publications arising from the trial before submission to journals, as will the AKTN Scientific Committee or its delegate.

Please see the AKTN Publication policy for more detail on authorship. 


\section{References}

[1] Allon M, Robbin ML. Increasing arteriovenous fistulas in hemodialysis patients: Problems and solutions. Kidney International. 2002 ; 62(4):1109-24.

[2] Polkinghorne KR, McDonald SP, Atkins RC, Kerr PG. Vascular Access and All-Cause Mortality: A Propensity Score Analysis. J Am Soc Nephrol. 2004; 15(2):477-86.

[3] Pastan S, Soucie JM, McClellan WM. Vascular access and increased risk of death among hemodialysis patients. Kidney International. 2002; 62(2):620-6.

[4] Excell L, Marshall M, McDonald SP. Haemodialysis, p35-52. In: ANZDATA Registry Report 2004. Adelaide, South Australia: Australia and New Zealand Dialysis and Transplant Registry; 2004.

[5] Manns B, Tonelli M, Yilmaz S, Lee H, Laupland K, Klarenbach S, et al. Establishment and Maintenance of Vascular Access in Incident Hemodialysis Patients: A Prospective Cost Analysis. J Am Soc Nephrol. 2005; 16(1):201-9.

[6] Port FK, Pisoni RL, Bommer J, Locatelli F, Jadoul M, Eknoyan G, et al. Improving Outcomes for Dialysis Patients in the International Dialysis Outcomes and Practice Patterns Study. Clin J Am Soc Nephrol. 2006; 1(2):246-55.

[7] Ishani A, Collins AJ, Herzog CA, Foley RN. Septicemia, access and cardiovascular disease in dialysis patients: The USRDS Wave 2 Study1. 2005; 68(1):311-8.

[8] Asif A, Roy-Chaudhury P, Beathard GA. Early Arteriovenous Fistula Failure: A Logical Proposal for When and How to Intervene. Clin J Am Soc Nephrol. 2006; 1(2):332-9.

[9] Rooijens PPGM, Tordoir JHM, Stijnen T, Burgmans JPJ, Smet de AAEA, Yo TI. Radiocephalic Wrist Arteriovenous Fistula for Hemodialysis: Meta-analysis Indicates a High Primary Failure Rate. European Journal of Vascular and Endovascular Surgery. 2004; 28(6):583-9.

[10] Marshall M, McDonald SP, Excell L, Livingston B, Shtangey V. Haemodialysis, p61-81. In: ANZDATA Registry Report 2005. Adelaide, South Australia: Australia and New Zealand Dialysis and Transplant Registry; 2005.

[11] Sidawy AN, Gray R, Besarab A, Henry M, Ascher E, Silva J, Michael, et al. Recommended standards for reports dealing with arteriovenous hemodialysis accesses. Journal of Vascular Surgery. 2002; 35(3):603-10.

[12] Andrassy K, Malluche $\mathrm{H}$, Bornefeld $\mathrm{H}$, Comberg M, Ritz E, Jesdinsky $\mathrm{H}$, et al. Prevention of p.o. clotting of av. cimino fistulae with acetylsalicyl acid. Results of a prospective double blind study. Klin Wochenschr. 1974; 52:348-9.

[13] Kooistra MP, van Es A, Marx JJM, Hertsig MLA, Struyvenberg A. Low-dose aspirin does not prevent thrombovascular accidents in low-risk haemodialysis patients during treatment with recombinant human erythropoietin. Nephrol Dial Transplant. 1994; 9(8):1115-20.

[14] Gröntoft K-C, Larsson R, Mulec H, Weiss LG, Dickinson JP. Effects of Ticlopidine in AVfistula Surgery in Uremia. Scandinavian Journal of Urology and Nephrology. 1998; 32(4):276-83.

[15] Kaufman JS. Antithrombotic Agents and the Prevention of Access Thrombosis. Seminars In Dialysis. 2000; 13(1):40-6.

[16] Engstrom K, Wallin R, Saldeen T. Effect of low-dose aspirin in combination with stable fish oil on whole blood production of eicosanoids. Prostaglandins, Leukotrienes and Essential Fatty Acids. 2001; 64(6):291-7.

AKTN Protocol - Favoured Study

Version 8.1 07/01/2010

Page 27 of 43 
[17] Schmitz PG, McCloud LK, Reikes ST, Leonard CL, Gellens ME. Prophylaxis of Hemodialysis Graft Thrombosis with Fish Oil: Double-Blind, Randomized, Prospective Trial. J Am Soc Nephrol. 2002; 13(1):184-90.

[18] Khajehdehi P. Lipid-lowering effect of polyunsaturated fatty acids in hemodialysis patients. Journal of Renal Nutrition. 2000; 10(4):191-5.

[19] Ando M, Sanaka T, Nihei H. Eicosapentanoic Acid Reduces Plasma Levels of Remnant Lipoproteins and Prevents in Vivo Peroxidation of LDL in Dialysis Patients. J Am Soc Nephrol. 1999 October 1, 1999; 10(10):2177-84.

[20] Beilin L, Mori T. Dietary w3 fatty acids. In: Whelton P, He J, Louis G, eds. Lifestyle Modification for the Prevention and Treatment of Hypertension. New York, USA: Marcel Dekker, Inc 2003:275-300.

[21] Mori T, Beilin L. w3 Fatty acids and inflammation. Curr Atheroscler Rep. 2004;6(6):461-7.

[22] Mori T. The effect of fish and fish oil - derived omega-3 fatty avids on lipid oxidation. Redox report. 2004; 9(4):193-7.

[23] Friedman A, Moe S. Review of the Effects of Omega-3 Supplementation in Dialysis Patients. Clin J Am Soc Nephrol. 2006; 1:182-92.

[24] Dember LM, Beck GJ, Allon M, Delmez JA, Dixon BS, Greenberg A, et al. Effect of clopidogrel on early failure of arteriovenous fistulas for hemodialysis: a randomized controlled trial. JAMA. 2008; 299(18):2164-71.

[25] Mori T, Bao D, Burke V, Puddey I, Watts G, O'Neal D, et al. Purified eicosapentaenoic acid and docosahexaenoic acid have differenceital effects on serum lipids and lipoproteins, LDL - particle size, glucose and insulin, in mildly hyperlipidaemic men. Am J Clin Nutr. 2000; 71:1085-94.

[26] Mori TA, Watts GF, Burke V, Hilme E, Puddey IB, Beilin LJ. Differential Effects of Eicosapentaenoic Acid and Docosahexaenoic Acid on Vascular Reactivity of the Forearm Microcirculation in Hyperlipidemic, Overweight Men. Circulation. 2000; 102(11):1264-9.

[27] Chan DC, Watts GF, Mori TA, Barrett PHR, Beilin LJ, Redgrave TG. Factorial study of the effects of atorvastatin and fish oil on dyslipidaemia in visceral obesity. European Journal of Clinical Investigation. 2002; 32(6):429-36.

[28] Woodman RJ, Mori TA, Burke V, Puddey IB, Watts GF, Beilin LJ. Effects of purified eicosapentaenoic and docosahexaenoic acids on glycemic control, blood pressure, and serum lipids in type 2 diabetic patients with treated hypertension. Am J Clin Nutr. 2002; 76(5):1007-15.

[29] Woodman RJ, Mori TA, Burke V, Puddey IB, Barden A, Watts GF, et al. Effects of purified eicosapentaenoic acid and docosahexaenoic acid on platelet, fibrinolytic and vascular function in hypertensive type 2 diabetic patients. Atherosclerosis. 2003; 166(1):85-93.

[30] de Abajo FJ, Garcia Rodriguez LA. Risk of upper gastrointestinal bleeding and perforation associated with low-dose aspirin as plain and enteric-coated formulations. BMC clinical pharmacology. 2001; 1:1-1.

[31] Livio M, Vigano G, Benigni A, Mecca G, Remuzzi G. Moderate doses of aspirin and risk of bleeding in renal failure. The Lancet. 1986; 327(8478):414-6.

[32] Gaspari F, Viganò G, Orisio S, Bonati M, Livio M, Remuzzi G. Aspirin prolongs bleeding time in uremia by a mechanism distinct from platelet cyclooxygenase inhibition. J Clin Invest. $1987 ; 79: 1788-97$.

AKTN Protocol - Favoured Study

Version 8.1 07/01/2010

Page 28 of 43 
[33] Baigent C, Landray M, Leaper C, Altmann P, Armitage J, Baxter A, et al. First United Kingdom Heart and Renal Protection (UK-HARP-I) study: biochemical efficacy and safety of simvastatin and safety of low-dose aspirin in chronic kidney disease. American Journal of Kidney Diseases. 2005; 45(3):473-84.

[34] Svaneborg N, Kristensen SD, Hansen LM, Bullow I, Husted SE, Schmidt EB. The acute and short-time effect of supplementation with the combination of n-3 fatty acids and acetylsalicylic acid on platelet function and plasma lipids. Thrombosis Research. 2002; 105(4):311-6.

[35] Diskin CJ, Thomas CE, Zellner CP, Lock S, Tanja J. Fish oil to prevent intimal hyperplasia and access thrombosis. Nephron. 1990; 55(4):445-7.

[36] Al-Harbi MM, Islam MW, Al-Shabanah OA, Al-Gharably NM. Effect of acute administration of fish oil (omega-3 marine triglyceride) on gastric ulceration and secretion induced by various ulcerogenic and necrotizing agents in rats. Food and Chemical Toxicology. 1995; 33(7):553-8.

[37] Szabo S, Rogers C. Diet, ulcer disease, and fish oil. Lancet. 1988; 331(8577):119.

[38] McDonald SP, Excell L, Shtangey V. Method and Location of Dialysis. ANZDATA Registry Report 2005. Adelaide: Australia and New Zealand Dialysis and Transplant Registry 2005:54-60.

[39] Robertson AJ, Nargund V, Gray DWR, Morris PJ. Low dose aspirin as prophylaxis against renal-vein thrombosis in renal-transplant recipients. Nephrol Dial Transplant. 2000; 15(11):1865-8.

[40] Murphy GJ, Taha R, Windmill DC, Metcalfe M, Nicholson ML. Influence of aspirin on early allograft thrombosis and chronic allograft nephropathy following renal transplantation. British Journal of Surgery. 2001; 88(2):261-6. 


\title{
13. Appendices
}

13.1 Sample Patient Information Sheet

(On institution letterhead)

(Name of Institution)

PATIENT INFORMATION SHEET

\section{A randomised, double-blind, placebo-controlled, factorial-design trial to assess the effect of aspirin and fish oil (omega-3 fatty acids) in the prevention of early thrombosis in arterio-venous fistulae in patients with Stage IV or V chronic kidney disease requiring haemodialysis}

\author{
FAVOURED Study (Fish oil and Aspirin in Vascular access OUtcomes In REnal Disease
}

\section{Research study:}

This document may help you to understand the information already discussed by your doctor regarding your participation in a clinical research study. It may assist you in deciding whether or not to take part- after knowing the possible risks and benefits of such a study. The information obtained from this study may possibly be helpful to others.

\section{Purpose of the study:}

The purpose of the study is to determine if aspirin and/or fish oil (omega-3 fatty acids) effectively reduces the early risk of blood clots in arterio-venous fistulae. (The arterio-venous fistula or AVF is the joined blood vessels or artery and vein which will be formed in your arm for haemodialysis.)

\section{Why is the study being performed?}

One problem which can occur with your AVF is thrombosis, that is, formation of a blood clot which blocks the blood vessel. If this happens, it will usually be within weeks of the procedure that created the AVF. One way to try and prevent this happening is to use medicines intended to prevent blocking of the blood vessel.

Platelets are cells in your blood which are involved in forming clots and aspirin has wellestablished anti-platelet effects. Omega-3 fatty acids have a number of biological effects which make it an attractive agent for the prevention of vascular access thrombosis. A combination of aspirin and omega-3 fatty acids should result in a more favourable effect on platelet aggregation than either agent used alone.

Who will be asked to enter the study?

You will be invited to participate in the trial if you will shortly having an AVF created in your arm and are over 19 years old.

What will happen on this study?

Visit 1 - Baseline Visit (up to 4 weeks prior to surgery)

At this visit, you will be asked to provide written informed consent to participate in the study. Details such as height, weight, blood pressure, relevant medical history (including past use of dialysis and other medical conditions) will be recorded. Details of any medications you currently take will be recorded. A fasting blood sample will be taken. You will be asked to complete a questionnaire about your lifestyle and dietary habits. 
Visit 2 - Randomisation Visit (up to 7 days prior to surgery)

At this visit, you will be given the study medication. You will be asked to take study medication every day from the day before your AVF procedure for 3 months. The medication will be low dose $(100 \mathrm{mg})$ aspirin tablet or its placebo and 4 capsules (4 grams) of omega- 3 fatty acids or its placebo. This allows 4 possible combinations of active medication or placebo (the placebos will look like the study medication but do not contain any active ingredients.) These amounts have proven to be effective in other studies. You will be asked to take 2 capsules and one tablet just before your breakfast and 2 capsules just before your evening meal. Which medication you receive will be determined by chance: you will have a 1 in 4 chance of receiving aspirin and omega-3 fatty acids together, aspirin alone, omega-3 fatty acids alone or placebo. Neither you, any of the study staff nor any member of your usual health care team will know which medication you are given.

While you are taking the study medication, please do not take any other medication which contains aspirin or fish oil (omega-3 fatty acids). Medications which contain aspirin include: Alka Seltzer, Asasantin, Aspalgin, Aspro, Astrix, Bayer, Aspirin, Cardiprin, Cartia, Codiphen, Codis, Codox, Codral, Disprin, Ecotrin, Solprin and Veganin. If you have a question about a medication you think may contain aspirin or omega-3 fatty acids, please contact your trial coordinator (insert name and phone number.)

Please also be aware that some herbal supplements have been reported to have similar effects to aspirin. Please be cautious when taking herbal supplements while you are also taking the study medication. Limited alcohol intake is also recommended while you are taking the study medication.

Visit 3 - AVF Surgery (1 day after starting medication)

On the day of your AVF procedure, a description of the procedure will be collected including the results of any vein mapping studies undertaken prior to surgery. Details such as height, weight, blood pressure, any changes to concomitant medicine or health will be reported.

Visits 4, 5 \& 6 - Treatment Visits (Weeks 1, 6 \& 12)

At these visits, details such as weight, blood pressure, information about your use of the study medication, your dialysis and AVF status, and any changes to your health or concomitant medications will be recorded. At visit 5 and 6 (weeks 6 and 12) a blood sample will be collected (you will need to fast for the week 12 sample).

Visit 7 onwards - Follow-up Visits (Month 6 onwards)

In this part of the study, you will have 6 monthly visits for up to 3 years. At these visits, details such as weight, blood pressure, your dialysis and AVF status and at month 6 any changes to your health or concomitant medications will be recorded.

Throughout the study, staff will regularly check your AVF for "bruit", the sound the blood normally makes if the AVF is working properly. No other aspect of your medical care will be altered in any way. You will still be looked after by your usual health care team. A summary of all data that will be recorded is in the table below.

AKTN Protocol - Favoured Study

Version 8.1 07/01/2010

Page 31 of 43 


\begin{tabular}{|l|c|c|c|c|c|c|c|c|c|c|c|c|}
\hline \multicolumn{1}{|c|}{ Study Phase } & Baseline & $\begin{array}{c}\text { Randomis } \\
\text { ation }\end{array}$ & Surgery & \multicolumn{6}{c|}{$\begin{array}{c}\text { Treatment } \\
\text { Period (wks) }\end{array}$} & \multicolumn{7}{c|}{ Follow-up (mths) } \\
\hline Assessment & & & & $\mathbf{1}$ & $\mathbf{6}$ & $\mathbf{1 2}$ & $\mathbf{6}$ & $\mathbf{1 2}$ & $\mathbf{1 8}$ & $\mathbf{2 4}$ & $\mathbf{3 0}$ & $\mathbf{3 6}$ \\
\hline $\begin{array}{l}\text { Study medication } \\
\text { dispensed }\end{array}$ & & $\mathrm{X}$ & & & & & & & & & & \\
\hline Informed consent & $\mathrm{X}$ & & & & & & & & & & & \\
\hline Medical history & $\mathrm{X}$ & & & & & & & & & & & \\
\hline Weight & $\mathrm{X}$ & & $\mathrm{X}$ & $\mathrm{X}$ & $\mathrm{X}$ & $\mathrm{X}$ & $\mathrm{X}$ & $\mathrm{X}$ & $\mathrm{X}$ & $\mathrm{X}$ & $\mathrm{X}$ & $\mathrm{X}$ \\
\hline Blood pressure & $\mathrm{X}$ & & $\mathrm{X}$ & $\mathrm{X}$ & $\mathrm{X}$ & $\mathrm{X}$ & $\mathrm{X}$ & $\mathrm{X}$ & $\mathrm{X}$ & $\mathrm{X}$ & $\mathrm{X}$ & $\mathrm{X}$ \\
\hline Demographics & $\mathrm{X}$ & & & & & & & & & & & \\
\hline Physical exam & $\mathrm{X}$ & & & & & & & & & & & \\
\hline Dialysis details & $\mathrm{X}$ & & & $\mathrm{X}$ & $\mathrm{X}$ & $\mathrm{X}$ & $\mathrm{X}$ & $\mathrm{X}$ & $\mathrm{X}$ & $\mathrm{X}$ & $\mathrm{X}$ & $\mathrm{X}$ \\
\hline $\begin{array}{l}\text { Description and status } \\
\text { of vascular access }\end{array}$ & $\mathrm{X}$ & & $\mathrm{X}$ & $\mathrm{X}$ & $\mathrm{X}$ & $\mathrm{X}$ & $\mathrm{X}$ & $\mathrm{X}$ & $\mathrm{X}$ & $\mathrm{X}$ & $\mathrm{X}$ & $\mathrm{X}$ \\
\hline Concomitant meds & $\mathrm{X}$ & & $\mathrm{X}$ & $\mathrm{X}$ & $\mathrm{X}$ & $\mathrm{X}$ & $\mathrm{X}$ & & & & & \\
\hline Adverse events & $\mathrm{X}$ & & $\mathrm{X}$ & $\mathrm{X}$ & $\mathrm{X}$ & $\mathrm{X}$ & $\mathrm{X}$ & & & & & \\
\hline Blood test (fasting) & $\mathrm{X}$ & & & & $\mathrm{X}$ & $\mathrm{X}$ & & & & & & \\
\hline
\end{tabular}

Are there any risks?

There is a large amount of clinical experience with the use of aspirin for prevention of blood clots. In general, aspirin is highly effective in preventing blood clots and there is only a very small increased risk of bleeding associated with taking aspirin. This study uses low dose aspirin (100mg once a day). The risk of side effects is very small at this dose, although you will be monitored carefully throughout this study and should report any unusual bleeding immediately to your renal unit nurses or nephrologist. Symptoms that could have serious consequences include black stools, feeling faint, signs of allergic reactions and abdominal pain.

Omega-3 fatty acids appear to be well tolerated, with gastrointestinal complaints particularly nausea, vomiting, diarrhoea and non-specific discomfort being the most often reported. These can be reduced by taking the medications with a glass of cold water. The combination of aspirin and omega- 3 fatty acids may be expected to be additive in terms of efficacy in vascular access thrombosis, but to have a lower risk of bleeding complications than the use of aspirin alone.

If you are a woman who is able to become pregnant, it is expected that you will use an effective method of birth control for the three months that you are taking the study medication. If you become pregnant during the study medication period, you should discontinue study medications and inform your renal unit nurses or nephrologist immediately. There is no evidence that the study medications affect men fathering children.

\section{Potential Benefits}

If you decide to participate in the trial, your condition will be very closely monitored. Any changes in your condition will be very quickly identified. The results of the study may also provide information, which could lead to improved use of AVFs for haemodialysis patients in the future. 


\section{Confidentiality}

All information gained from this study will be treated with utmost confidentiality without reference to your name. You will be asked to give permission to any regulatory authorities related to the study to access your medical records. The information will only be used for purposes of this study. Information gathered by the ANZDATA Registry (the Australia and New Zealand Dialysis and Transplant Registry) may also be used as part of this study. Medical records will be stored for accessibility for at least 15 years.

$\underline{\text { Costs }}$

Your participation in this study will not cost you any money. You will not receive any payment for participation in this study. Participation in this study will not change usual hospital liability arrangements and processes.

\section{Do you have a choice?}

Entry into this study is entirely voluntary. Choosing not to take part in this study will not affect your treatment or care in any way. The doctors will continue to treat you with the best means available.

If you agree to participate in this study you will be asked to sign a consent form. However you may withdraw from the study at any time without giving a reason. Your doctor may also decide that you should stop if they believe it is in your best interest. You will be told if any new information arises which might affect your decision to be in the study.

For further information about this study, please contact (medical contact, research nurse contact, phone numbers).

\section{Ethics Approval}

This study has been reviewed and approved by the (local) Ethics Committee. Should you wish to discuss the matter with someone not directly involved, you can contact (Ethics Committee contact, number).

This study has been cleared by one of the human ethics committees of the University of Queensland in accordance with the National Health and Medical Research Council's guidelines. You are of course, free to discuss your participation in this study with project staff (contactable on ......................). If you would like to speak to an officer of the University not involved in the study, you may contact the Ethics Officer on 0733653924. 
13.2 Sample Patient Consent Form

(Name of institution)

Consent Form

A randomised, double-blind, placebo-controlled, factorial-design trial to assess the effect of aspirin and fish oil (omega- 3 fatty acids) in the prevention of early thrombosis in arterio-venous fistulae in patients with Stage IV or V chronic kidney disease requiring haemodialysis

FAVOURED Study (Fish oil and Aspirin in Vascular access OUtcomes In REnal Disease

- I agree to participate in the above named clinical trial.

- I have read and understood and kept a copy of the Patient Information Sheet.

- I acknowledge and understand the methods and demands relating to the study and the possible risks and inconveniences which may occur.

- I have had my questions answered to my satisfaction.

- I understand that I may withdraw from the study at any time without giving any reason and that this will not affect my medical treatment.

- I give my permission for access to my medical records including information gathered by the ANZDATA Registry (the Australia and New Zealand Dialysis and Transplant Registry) for the purposes of this research.

- I give my permission for access to my medical records for the purposes of this research, including the pre-surgical Doppler vein mapping.

\begin{tabular}{lll}
\hline Patient's signature & Printed name
\end{tabular}

(to be personally dated)

\begin{tabular}{lcc}
\hline Witness signature & Printed name & Date \\
(to be personally dated)
\end{tabular}

I have explained the nature and purpose of this study to the above participant and have answered their questions.

Investigator's signature

Printed name

Date

AKTN Protocol - Favoured Study

Version 8.1 07/01/2010

Page 34 of 43 


\subsection{Classification of Chronic Kidney Disease}

The Kidney Disease Outcomes Quality Initiative (K/DOQI) of the National Kidney Foundation (NKF) defines chronic kidney disease (CKD) as either kidney damage or a decreased kidney glomerular filtration rate (GFR) of $<60 \mathrm{~mL} / \mathrm{min} / 1.73 \mathrm{~m}^{2}$ for 3 or more months. February 2002 $\mathrm{K} / \mathrm{DOQI}$ classification of the stages of CKD:

- Stage 1: Kidney damage with normal or increased GFR (>90 mL/min/1.73 $\mathrm{m}^{2}$ )

- Stage 2: Mild reduction in GFR $\left(60-89 \mathrm{~mL} / \mathrm{min} / 1.73 \mathrm{~m}^{2}\right)$

- Stage 3: Moderate reduction in GFR $\left(30-59 \mathrm{~mL} / \mathrm{min} / 1.73 \mathrm{~m}^{2}\right)$

- Stage 4: Severe reduction in GFR $\left(15-29 \mathrm{~mL} / \mathrm{min} / 1.73 \mathrm{~m}^{2}\right)$

- Stage 5: Kidney failure (GFR $<15 \mathrm{~mL} / \mathrm{min} / 1.73 \mathrm{~m}^{2}$ or dialysis) 


\subsection{Lifestyle questionnaire}

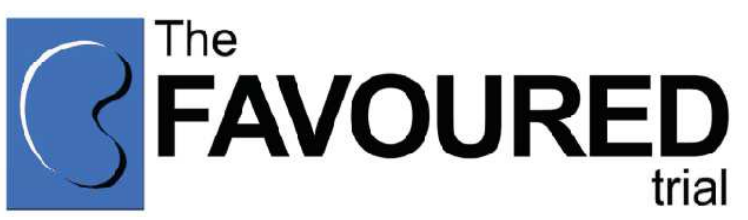

\section{LIFESTYLE QUESTIONNAIRE}

\section{Fish Oil and Aspirin in the Prevention of Thrombosis in Chronic Kidney Disease}

Thank you for your interest in participating in this research trial. We would very much appreciate your help in filling in this questionnaire, which will give us information about your lifestyle, including diet and exercise. Once you have completed the questionnaire please return it to the supervising research staff member.

The questionnaire should take approximately 15 minutes to complete.

Please take your time and answer all questions as accurately as possible.

\section{N.B. ALL INFORMATION WILL REMAIN STRICTLY CONFIDENTIAL}

Today's Date $(\mathrm{dd} / \mathrm{mm} / \mathrm{yyyy})$ 1

Patient's Initials

Date of Birth (dd/mm/yyyy) ' 


\section{YOUR DRINKING DETAILS}

1. Do you drink alcohol? (Circle one)

I have never drunk alcohol (go to Question 4) 1

I used to drink alcohol (go to Question 2) . . . . 2

I occasionally drink alcohol (go to Question 3) . . 3

2. If you gave up drinking alcohol, how long ago did you stop? (Circle one)

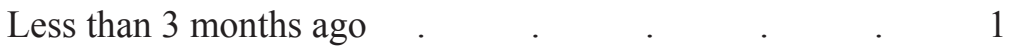

3 - 6 months ago . . . . . . . . . 2

More than 6 months but less than 1 year ago . . 3

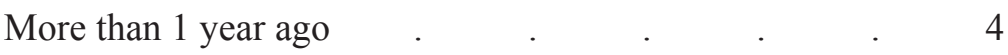

(go to Question 4)

3. How many standard drinks (see below) of alcohol do you usually drink? (Circle one)

\begin{tabular}{|l|c|c|c|c|c|c|}
\hline & $\begin{array}{c}\text { Less than 1 } \\
\text { per week }\end{array}$ & $\begin{array}{c}1-6 \text { per } \\
\text { week }\end{array}$ & 1 per day & $2-3$ per day & 4 per day & $\begin{array}{c}\text { More than 4 } \\
\text { per day }\end{array}$ \\
\hline A. Beer & 1 & 2 & 3 & 4 & 5 & 6 \\
\hline B. Wine & 1 & 2 & 3 & 4 & 5 & 6 \\
\hline C. Spirits & 1 & 2 & 3 & 4 & 5 & 6 \\
\hline
\end{tabular}

\section{DO YOU KNOW A STANDARD DRINK WHEN YOU SEE ONE?}

To help you keep count of your drinks, remember any drink containing about 10 grams of alcohol is called a standard drink. For example:

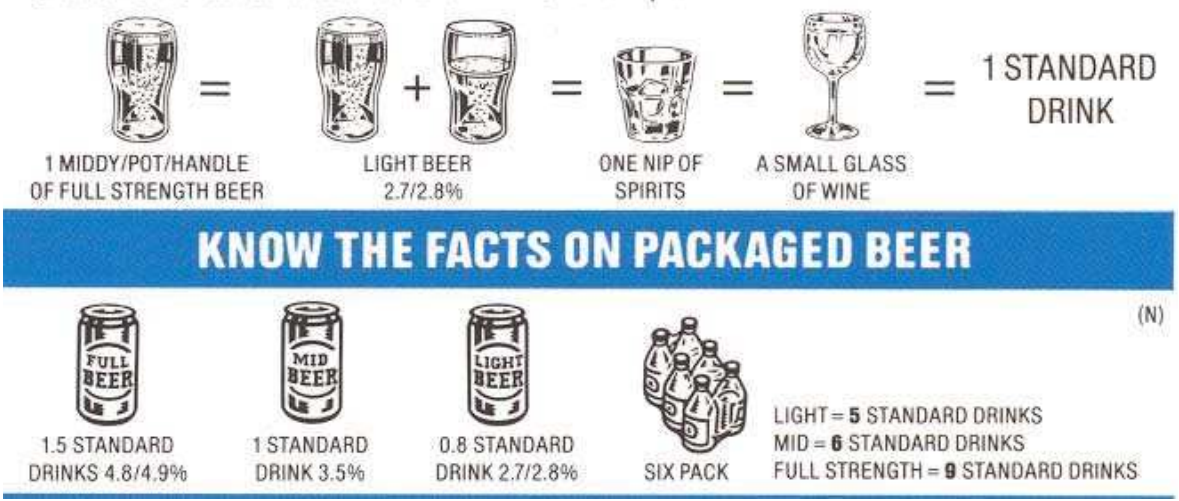




\section{YOUR SMOKING HISTORY}

4. Do you smoke cigarettes, cigars, pipes or other tobacco products? (Circle one)

I have never smoked (go to Question 7) $\quad$. $\quad . \quad$. $\quad 1$

I used to smoke (go to Question 5) . $\quad$. $\quad . \quad$. $\quad$. 2

I am currently a smoker (go to Question 6) . . . $\quad 3$

5. If you gave up smoking, about how long ago did you stop? (Circle one)

Less than 3 months ago $\quad . \quad$. $\quad$. $\quad$. $\quad$. 1

$3-6$ months ago . . . . . . . . . $\quad$. 2

More than 6 months but less than 1 year ago . . 3

More than 1 year ago . . . . . . . . 4

(go to Question 7)

6. If you smoke, how many cigarettes, cigars, etc do you smoke per day on average?

per day

\section{YOUR LEVEL OF PHYSICAL ACTIVITY}

7. How often do you exercise (includes taking walks, gardening or playing sport)? (Circle one)

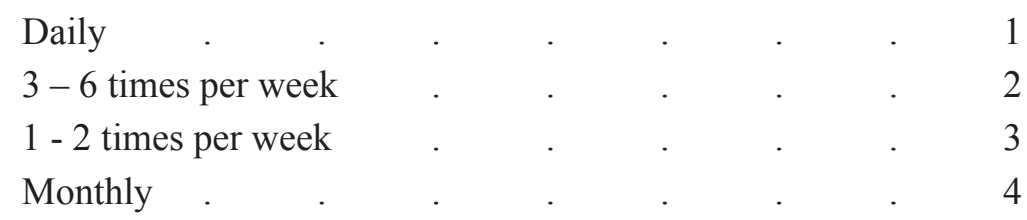

8. How long, on average, are you active for on these days?

min per day

\section{$\underline{\text { YOUR DIET }}$}

9. How many serves of vegetables or salad do you eat per day on

per day

average? (A serve is half-a cup)

10. How many serves of fruit do you eat per day on average? (A serve is half-a cup or the equivalent of one medium apple)

per day

11. How many times per week do you eat fried food?

per week

12. How many times per week do you eat pies, cakes, biscuits or per week chocolate? 
13. How often do you eat fish on average? (Circle one)

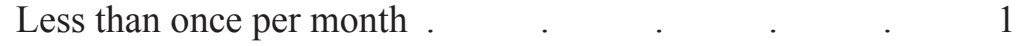

1 - 3 times per month . . . . . . . . 2

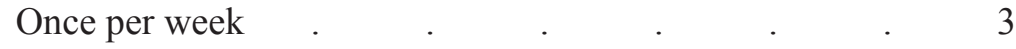

2 - 4 times per week $\quad . \quad$. $\quad . \quad$. $\quad . \quad . \quad 4$

More than 5 times per week $\quad$. $\quad . \quad$. $\quad$. 5

14. What type of fish do you usually eat? (Circle one)

Fish, grilled, steamed, or baked . . . . . . . 1

Fish, fried . $\quad . \quad$. $\quad$. $\quad$. $\quad$. $\quad$. 2

Fish, canned (include tuna, salmon, sardines, mackerel) . $\quad 3$

Shellfish (include prawns, scallops, crayfish) 4

15. If you have recently taken fish oil capsules, on average, how many did you take?

per day

16. How often do you eat red meat on average? (Circle one)

Less than once per month . $\quad$. $\quad$. $\quad$. $\quad$. 1

1 - 3 times per month $\quad . \quad$. $\quad . \quad$. $\quad . \quad 2$

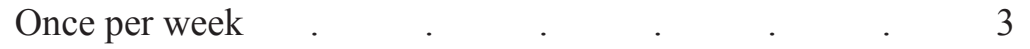

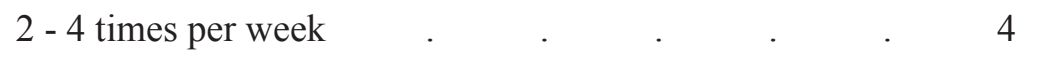

More than 5 times per week $\quad$. $\quad$. $\quad$. $\quad$. 5

17. When you red eat meat do you remove the fat? (Circle one)

Usually . . . . . . . . . . 1

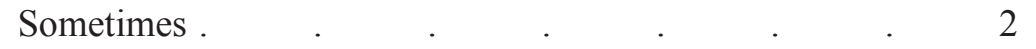

Never / rarely $\quad . \quad . \quad . \quad . \quad . \quad . \quad . \quad 3$

18 Do you add salt to food at the table? (Circle one)

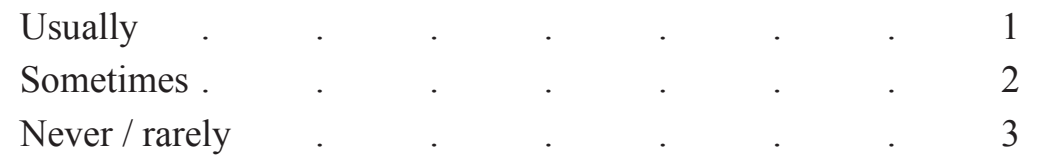

19. How often do you eat chicken on average? (Circle one)

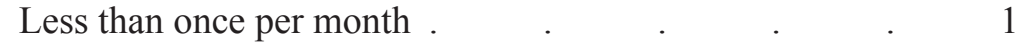

1 - 3 times per month . . . . . . . 2

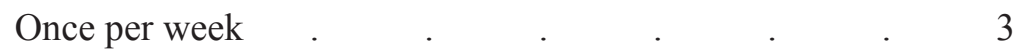

2 - 4 times per week $\quad . \quad$. $\quad . \quad$. $\quad . \quad 4$

More than 5 times per week $\quad$. $\quad$. $\quad . \quad$. 5 
20. When you eat chicken do you remove the skin? (Circle one)

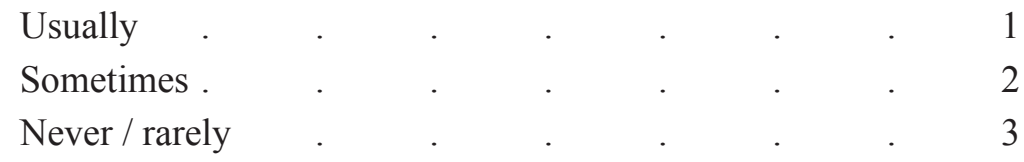

21. What kind of milk do you usually use? (Circle one)

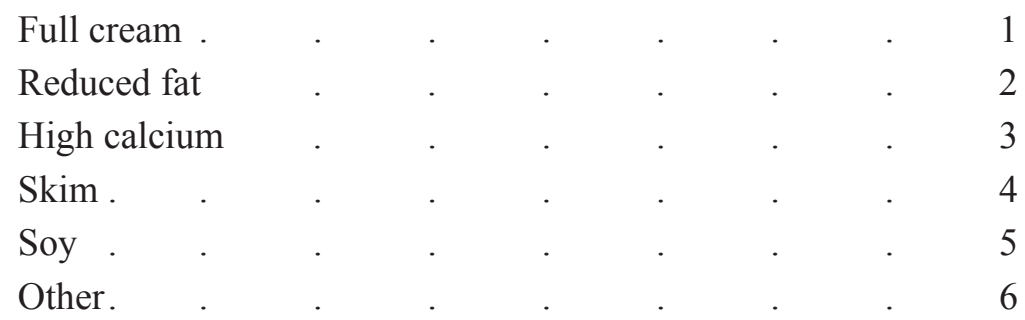

If other, please specify

22. How much milk do you consume per day?

1 cup of coffee or tea has the equivalent of 1 tablespoon of milk

tablespoons per day

1 bowl of cereal has the equivalent of $1 / 2$ cup of milk

cups per day

\section{YOUR FAMILY HISTORY}

23. Did your father have cardiovascular disease (stroke or other disease affecting the heart or blood vessels) before he was 45 years old? (Circle one)

$\begin{array}{llllll}\text { Yes . } & \cdot & \cdot & . & . & 1 \\ \text { No } . & . & . & . & . & 2 \\ \text { Don't know. } & . & . & . & . & 3\end{array}$

24. Did your mother have cardiovascular disease before she was 55 years old? (Circle one)

$\begin{array}{llllll}\text { Yes . } & \cdot & \cdot & \cdot & \cdot & 1 \\ \text { No } \cdot & \cdot & \cdot & \cdot & \cdot & 2 \\ \text { Don't know. } & \cdot & . & . & . & 3\end{array}$

\section{THANK YOU FOR YOUR CO-OPERATION}




\subsection{Data related to patency rates}

Table: Studies comparing long-term patency AVF versus AVG.

\begin{tabular}{|c|c|c|c|c|c|c|c|c|c|c|}
\hline Study & $\begin{array}{c}\text { Study } \\
\text { Type }\end{array}$ & Country & $\begin{array}{c}\text { Access } \\
\text { Type }\end{array}$ & Number & $\begin{array}{c}1^{\circ} \text { Failure } \\
(\%)\end{array}$ & $\begin{array}{c}1^{\circ} \text { Patency } \\
1 \text { yr (\%) }\end{array}$ & $\begin{array}{c}1^{\circ} \text { Patency } \\
2 y r(\%)\end{array}$ & $\begin{array}{c}1^{\circ} \text { Patency } \\
\text { 3yr (\%) }\end{array}$ & $\begin{array}{l}1^{\circ} \text { failure } \\
\text { included }\end{array}$ & $\begin{array}{c}\text { Intervention } \\
\text { Rate } \\
\text { (/acc-yr) }\end{array}$ \\
\hline \multirow{2}{*}{ Palder 1985} & \multirow{2}{*}{$\mathrm{R}, \mathrm{S}$} & \multirow{2}{*}{ USA } & $\mathrm{RCF}$ & 154 & 24 & $60^{*}$ & $50^{*}$ & $42^{*}$ & $\mathrm{Y}$ & ** \\
\hline & & & AVG & 163 & NS & $78^{*}$ & $70^{*}$ & $56^{*}$ & Y & ** \\
\hline \multirow{2}{*}{ Winsett 1985} & \multirow{2}{*}{$\mathrm{R}, \mathrm{S}$} & \multirow{2}{*}{ USA } & RCF & 273 & 27 & & & $72\left(84^{*}\right)$ & $\mathrm{N}$ & 0.06 \\
\hline & & & AVG & 202 & NS & & & $39\left(70^{*}\right)$ & $\mathrm{N}$ & 0.44 \\
\hline \multirow{2}{*}{ Kherlakian 1986} & \multirow{2}{*}{$\mathrm{R}, \mathrm{S}$} & \multirow{2}{*}{ USA } & RCF & 100 & 12 & $71^{*}$ & $66^{*}$ & $64^{*}$ & $\mathrm{Y}$ & $* * *$ \\
\hline & & & AVG & 100 & 4 & $75^{*}$ & $61^{*}$ & $50^{*}$ & $Y$ & $* * *$ \\
\hline \multirow{2}{*}{ Zibari 1988} & \multirow{2}{*}{$\mathrm{R}, \mathrm{S}$} & \multirow{2}{*}{ USA } & AVF & 160 & & & & & & $\star * * *$ \\
\hline & & & AVG & 166 & & & & & & $* * *$ \\
\hline \multirow{2}{*}{ Coburn 1994} & \multirow{2}{*}{$\mathrm{R}, \mathrm{S}$} & \multirow{2}{*}{ USA } & Basilic & 59 & NS & $90\left(90^{\star}\right)$ & $86\left(86^{*}\right)$ & & NS & $\star \star \star *$ \\
\hline & & & AVG & 47 & NS & $70\left(87^{*}\right)$ & $49\left(64^{*}\right)$ & & NS & $* * *$ \\
\hline \multirow{2}{*}{ Burger 1995} & \multirow{2}{*}{$\mathrm{P}, \mathrm{S}^{\wedge}$} & \multirow{2}{*}{ Netherlands } & RCF & 208 & NS & $53\left(79^{\star}\right)$ & $36\left(68^{\star}\right)$ & $24\left(59^{\star}\right)$ & NS & NS \\
\hline & & & AVG & 46 & NS & $37\left(61^{*}\right)$ & $21\left(60^{*}\right)$ & $17\left(54^{*}\right)$ & NS & NS \\
\hline \multirow{2}{*}{ Enzler 1996} & \multirow{2}{*}{$\mathrm{R}, \mathrm{S}$} & \multirow{2}{*}{ Switzerland } & RCF & 429 & NS & $70\left(74^{\star}\right)$ & $61\left(67^{\star}\right)$ & $59\left(64^{\star}\right)$ & NS & ** \\
\hline & & & AVG & 69 & NS & $41\left(58^{\star}\right)$ & $24\left(47^{\star}\right)$ & $24\left(40^{*}\right)$ & NS & ** \\
\hline \multirow{2}{*}{ Rocco 1996} & \multirow{2}{*}{$\mathrm{R}, \mathrm{S}$} & \multirow{2}{*}{ USA } & RCF & 48 & 31 & $55\left(78^{\star}\right)$ & & & NS & 0.34 \\
\hline & & & AVG & 40 & 12 & $60\left(88^{*}\right)$ & & & NS & 0.8 \\
\hline \multirow{2}{*}{ Miller 1997} & \multirow{2}{*}{$\mathrm{R}, \mathrm{S}$} & \multirow{2}{*}{ USA } & AVF & 75 & NS & $84\left(83^{\star}\right)$ & $74\left(70^{*}\right)$ & & NS & 0.17 \\
\hline & & & AVG & 23 & NS & $47\left(73^{*}\right)$ & $38\left(58^{*}\right)$ & & NS & 1.17 \\
\hline \multirow{2}{*}{ Hodges 1997} & \multirow{2}{*}{$\mathrm{R}, \mathrm{S}$} & & AVF & 87 & 31 & $43\left(46^{\star}\right)$ & & & $\mathrm{Y}$ & 0.07 \\
\hline & & USA & AVG & 236 & NS & $41\left(59^{*}\right)$ & & & $Y$ & 0.5 \\
\hline & & & AVF & 108 & 8 & 83 & & & $Y$ & NS \\
\hline Silva 1998 & $\mathrm{P}, \mathrm{S}$ & USA & AVG & 52 & 8 & 74 & & & $Y$ & NS \\
\hline & BS & ISA & Basilic & 30 & NS & & $70\left(70^{*}\right)$ & & NS & ** \\
\hline Mlatsuura 1998 & $\mathrm{R}, \mathrm{S}$ & USA & AVG & 68 & NS & & $46\left(51^{*}\right)$ & & NS & ** \\
\hline & & & AVF & 235 & 12 & & $54\left(70^{*}\right)$ & & $Y$ & ** \\
\hline Kalman 1999 & P,S & canada & AVG & 231 & NS & & $18\left(60^{*}\right)$ & & $Y$ & ** \\
\hline Accher 2000 & $B S$ & HSA & AVF & 99 & 18 & 84 & & & $\mathrm{~N}$ & 0.07 \\
\hline Ascrier zuOU & గ, ১ & USA & AVG & 122 & NS & 54 & & & $\mathrm{~N}$ & 0.74 \\
\hline
\end{tabular}

AKTN Protocol - Favoured Study

Version 8.1 07/01/2010
Page 41 of 43 


\begin{tabular}{|c|c|c|c|c|c|c|c|c|c|c|}
\hline Study & $\begin{array}{c}\text { Study } \\
\text { Type }\end{array}$ & Country & $\begin{array}{l}\text { Access } \\
\text { Type }\end{array}$ & Number & $\begin{array}{c}1^{\circ} \text { Failure } \\
(\%)\end{array}$ & $\begin{array}{c}1^{\circ} \text { Patency } \\
1 \mathrm{yr}(\%)\end{array}$ & $\begin{array}{c}1^{\circ} \text { Patency } \\
2 y r(\%)\end{array}$ & $\begin{array}{c}1^{\circ} \text { Patency } \\
\text { 3yr (\%) }\end{array}$ & $\begin{array}{l}1^{\circ} \text { failure } \\
\text { included }\end{array}$ & $\begin{array}{c}\text { Intervention } \\
\text { Rate } \\
\text { (acc/yr) }\end{array}$ \\
\hline \multirow{2}{*}{ Staramos $2000^{+}$} & \multirow{2}{*}{$\mathrm{R}, \mathrm{S}$} & \multirow{2}{*}{ Greece } & AVF & 68 & NS & $67\left(67^{\star}\right)$ & $52\left(52^{\star}\right)$ & $44\left(44^{\star}\right)$ & $Y$ & NS \\
\hline & & & AVG & 67 & NS & $66\left(81^{*}\right)$ & $53\left(65^{\star}\right)$ & $38\left(58^{*}\right)$ & $Y$ & NS \\
\hline \multirow{3}{*}{ Gibson 2001b } & \multirow{3}{*}{$\mathrm{P}, \mathrm{M}$} & \multirow{3}{*}{ USA } & AVF & 492 & NS & $56\left(73^{\star}\right)$ & $40\left(64^{*}\right)$ & & NS & $1.0^{\mathrm{C}}$ \\
\hline & & & VTAVF & 181 & NS & $44\left(68^{\star}\right)$ & $28\left(60^{\star}\right)$ & & NS & $1.32^{c}$ \\
\hline & & & AVG & 1574 & NS & $38\left(72^{*}\right)$ & $25\left(60^{*}\right)$ & & NS & $1.91^{c}$ \\
\hline \multirow{2}{*}{ Allon 2001} & \multirow{2}{*}{$\mathrm{R}, \mathrm{S}$} & \multirow{2}{*}{ USA } & AVF & 139 & 46 & $42\left(44^{*}\right)$ & & & $\mathrm{Y}$ & 0.57 \\
\hline & & & AVG & 78 & 21 & $43\left(48^{\star}\right)$ & & & $\mathrm{Y}$ & 1.67 \\
\hline \multirow{2}{*}{ Gibson 2001a } & \multirow{2}{*}{$\mathrm{R}, \mathrm{S}$} & \multirow{2}{*}{ USA } & AVF & 130 & 23 & $56\left(72^{*}\right)$ & $41\left(61^{*}\right)$ & & $\mathrm{Y}$ & $* *$ \\
\hline & & & AVG & 92 & NS & $36\left(58^{*}\right)$ & $11\left(41^{*}\right)$ & & Y & $* *$ \\
\hline \multirow{3}{*}{ Oliver 2001} & \multirow{3}{*}{$\mathrm{R}, \mathrm{S}$} & \multirow{3}{*}{ USA } & $\mathrm{BCF}$ & 56 & 32 & 64 & & & $\mathrm{~N}$ & 0.4 \\
\hline & & & Basilic & 59 & 21 & 64 & & & $\mathrm{~N}$ & 0.7 \\
\hline & & & AVG & 80 & 15 & 62 & & & $\mathrm{~N}$ & 2.4 \\
\hline \multirow{3}{*}{ Dixon 2002} & \multirow{3}{*}{$\mathrm{R}, \mathrm{S}$} & \multirow{3}{*}{ USA } & $\mathrm{RCF}$ & 88 & 32 & $44\left(52^{*}\right)$ & $40\left(48^{\star}\right)$ & $31\left(43^{\star}\right)$ & $\mathrm{Y}$ & 1.4 \\
\hline & & & $\mathrm{BCF}$ & 117 & 28 & $62\left(69^{*}\right)$ & $48\left(59^{*}\right)$ & $37\left(53^{*}\right)$ & $Y$ & 2.71 \\
\hline & & & AVG & 117 & 22.6 & $27\left(54^{*}\right)$ & $13\left(42^{*}\right)$ & $7\left(31^{*}\right)$ & $\mathrm{Y}$ & 3.84 \\
\hline \multirow{2}{*}{ Pisoni 2002} & \multirow{2}{*}{$\mathrm{P}, \mathrm{M}$} & \multirow[b]{2}{*}{ USA } & AVF & 177 & NS & 68 & & & $\mathrm{~N}$ & NS \\
\hline & & & AVG & 251 & NS & 49 & & & $\mathrm{~N}$ & NS \\
\hline \multirow{2}{*}{ Lawrence 2002} & \multirow{2}{*}{$\mathrm{R}, \mathrm{S}$} & \multirow{2}{*}{ Australia } & AVF & 64 & NS & 87 & 87 & & NS & NS \\
\hline & & & AVG & 26 & NS & 63 & 50 & & NS & NS \\
\hline \multirow{4}{*}{ Shenoy 2003} & \multirow{4}{*}{$\mathrm{R}, \mathrm{M}$} & \multirow{4}{*}{ USA } & $\mathrm{AVF}^{\#}$ & 242 & 17 & & $54\left(67^{\star}\right)$ & & $\mathrm{Y}$ & 0.22 \\
\hline & & & $\mathrm{AVG}^{\#}$ & 440 & 17 & & $36\left(39^{*}\right)$ & & $Y$ & 0.86 \\
\hline & & & AVF \# & 276 & 28 & & $34\left(48^{*}\right)$ & & $\mathrm{Y}$ & 0.37 \\
\hline & & & AVG \#\# & 384 & 28 & & $17\left(19^{\star}\right)$ & & Y & 1.73 \\
\hline \multirow{2}{*}{ Fischer 2003} & \multirow{2}{*}{$\mathrm{R}, \mathrm{S}$} & & RCF & 147 & NS & $69^{*}$ & $63^{*}$ & $55^{*}$ & NS & ** \\
\hline & & Australia & AVG & 50 & NS & $89^{*}$ & $75^{*}$ & $68^{*}$ & NS & ** \\
\hline & & & $\mathrm{BCF}$ & 30 & 27 & $53\left(67^{\star}\right)$ & $34\left(56^{*}\right)$ & & $\mathrm{Y}$ & ** \\
\hline Choi 2003 & $\mathrm{R}, \mathrm{S}$ & USA & Basilic & 42 & 5 & $76\left(83^{*}\right)$ & $68\left(75^{\star}\right)$ & & $\mathrm{Y}$ & $* *$ \\
\hline & & & AVG & 53 & NS & $47\left(59^{*}\right)$ & $26\left(40^{\star}\right)$ & & $Y$ & $* *$ \\
\hline
\end{tabular}

AKTN Protocol - Favoured Study

Version 8.1 07/01/2010

Page 42 of 43 


\begin{tabular}{|c|c|c|c|c|c|c|c|c|c|c|}
\hline Study & $\begin{array}{l}\text { Study } \\
\text { Type }\end{array}$ & Country & $\begin{array}{c}\text { Access } \\
\text { Type }\end{array}$ & Number & $\begin{array}{c}10 \text { Failure } \\
(\%)\end{array}$ & $\begin{array}{c}\text { 10 Patency } \\
\text { 1yr (\%) }\end{array}$ & $\begin{array}{c}\text { 10 Patency } \\
\text { 2yr (\%) }\end{array}$ & $\begin{array}{c}\text { 10 Patency } \\
\text { 3yr (\%) }\end{array}$ & $\begin{array}{l}10 \text { failure } \\
\text { included }\end{array}$ & $\begin{array}{c}\text { Intervention } \\
\text { Rate } \\
\text { (acc/yr) }\end{array}$ \\
\hline \multirow{2}{*}{ Huber 2003} & \multirow{2}{*}{ Meta } & \multirow{2}{*}{ NA } & AVF \#\# & 1849 & NS & $72^{\mathrm{a}}\left(86^{\star}\right)$ & $51^{\mathrm{b}}\left(77^{\star}\right)$ & & NS & NS \\
\hline & & & AVG & 1245 & NS & $58^{\mathrm{a}}\left(76^{*}\right)$ & $33^{b}\left(55^{\star}\right)$ & & NS & NS \\
\hline \multirow{2}{*}{ Perara 2004} & \multirow{2}{*}{$\mathrm{R}, \mathrm{S}$} & \multirow{2}{*}{ USA } & AVF & 100 & $11 \%$ & $56\left(64^{*}\right)$ & $39\left(53^{*}\right)$ & & $\mathrm{Y}$ & 0.53 \\
\hline & & & AVG & 131 & NS & $36\left(65^{\star}\right)$ & $9\left(46^{\star}\right)$ & & $\mathrm{Y}$ & 0.92 \\
\hline \multirow{4}{*}{ Kawecka 2005} & \multirow{4}{*}{$\mathrm{R}, \mathrm{S}$} & \multirow{4}{*}{ Poland } & RCF & 540 & NS & $52\left(67^{\star}\right)$ & $41\left(56^{*}\right)$ & & NS & NS \\
\hline & & & $\mathrm{BCF}$ & 143 & NS & $45\left(61^{*}\right)$ & $31\left(44^{*}\right)$ & & NS & NS \\
\hline & & & Basilic & 85 & NS & $42\left(54^{\star}\right)$ & $28\left(38^{*}\right)$ & & NS & NS \\
\hline & & & AVG & 86 & NS & $48\left(56^{*}\right)$ & $21\left(33^{*}\right)$ & & NS & NS \\
\hline \multirow{2}{*}{ Fitzgerald 2005} & \multirow{2}{*}{$\mathrm{R}, \mathrm{S}$} & \multirow{2}{*}{ USA } & UAAVF & 86 & NS & 50 & 46 & & NS & ** \\
\hline & & & AVG & 60 & NS & 50 & 37 & & NS & ** \\
\hline
\end{tabular}

Table from Polkinghorne, unpublished

\section{Footnotes:}

$1^{\circ}=\quad$ Primary

AVF $=$ native arteriovenous fistula

$\mathrm{RCF}=$ radiocephalic $\mathrm{AVF}$

$\mathrm{BCF}=$ Brachipephalic AVF

Basilic = Brachiobasilic AVF

UAAVF = Upper Arm AVF (either BCF or Brachiobasilic AVF)

$\mathrm{AVG}=$ arteriovenous (PTFE) graft

AVG $=$ arteriovenous (PTFE) graft
VTAVF $=$ Venous transposition AVF

$\mathrm{R}=\quad$ Retrospective

$\begin{array}{ll}\mathrm{R}= & \text { Retrospective } \\ \mathrm{P}= & \text { Prospective }\end{array}$

$\begin{array}{ll}\mathrm{P}= & \text { Prospective } \\ \mathrm{M}= & \text { Single centre }\end{array}$

$\mathrm{M}=\quad$ multicentre

Meta $=$ meta-analysis

$\mathrm{Y}=\quad$ Yes
$\mathrm{N}=\quad$ No

$\mathrm{NS}=\quad$ Not stated

$\mathrm{NA}=\quad$ Not applicable

* Indicates secondary patency,

** Overall rates not given however significantly more thrombectomies and revisions in the AVG group

*** Overall rates not given however significantly more complications seen in the AVG group

$\wedge$ Retrospective 1971-1980, prospective 1980-1991

\# Clips used

\#\# Clips used

\#\#\# Upper arm AVF only + only patients 70 years and over

${ }^{a} 6$ month patency

tency

${ }^{c}$ Incident Rate Ratio, AVF comparison group VTAVF vs AVG $p=0.04$, AVG vs AVF $p<0.01$
AKTN Protocol - Favoured Study

Version 8.1 07/01/2010
Page 43 of 43 
A randomised, double-blind, placebo-controlled, factorial-design trial to assess the effect of aspirin and fish oil (omega-3 fatty acids) in the prevention of early thrombosis in arterio-venous fistulae in patients with Stage IV or V chronic kidney disease requiring haemodialysis

FAVOURED (Fish oil and Aspirin in Vascular access OUtcomes in REnal Disease)

\begin{tabular}{ll} 
& \multicolumn{1}{c}{ Trial Protocol } \\
$\begin{array}{l}\text { Principal Investigator } \\
\text { Dr Ashley Irish }\end{array}$ & $\begin{array}{l}\text { Version 9, 30 November } 2010 . \\
\end{array}$ \\
& $\begin{array}{l}\text { Renal Unit, Royal Perth Hospital, Perth } \\
\text { phone: 61 8 9224 2546 } \\
\text { Ashley.Irish@health.wa.gov.au }\end{array}$
\end{tabular}

Trial Management Committee

Dr Alan Cass

The George Institute for International Health, Sydney, Australia

Dr Vlado Perkovic

Dr Sharan Dogra

The George Institute for International Health, Sydney, Australia

Elaine Beller

Sir Charles Gardiner Hospital, Perth, Australia

Ass Prof Carmel Hawley

Professor Peter Kerr

Dr Trevor Mori

Dr Kevan Polkinghorne

Dr Amanda Robertson

Dr David Voss

Elvie Haluszkiewicz

Dr David Gracey

Dr Amanda Mather

Dr Stephen McDonald

Dr Hooi Lai Seong

Dr Colin Hutchison

Dr Chris Mclntyre University of Queensland, Brisbane, Australia

Princess Alexandra Hospital, Brisbane, Australia

Monash Medical Centre, Melbourne, Australia

University of Western Australia, Perth, Australia

Monash Medical Centre, Melbourne, Australia

Royal Melbourne Hospital, Melbourne, Australia

Middlemore Hospital, Auckland, New Zealand

Royal Perth Hospital, Perth, Australia

Royal Prince Alfred Hospital, Sydney, Australia

Royal North Shore Hospital, Sydney, Australia

Royal Adelaide Hospital, Adelaide, Australia

Hospital Sultanah Aminah, Johor Bahru, Malaysia

Queen Elizabeth Hospital, Birmingham, United Kingdom

Derby City Hospital, Derby, United Kingdom

Peta-Anne Paul-Brent

University of Queensland, Brisbane, Australia

Trial Statisticians

Charles Thompson

University of Queensland, Brisbane, Australia

Stephane Heritier

The George Institute for International Health, Sydney, Australia

AKTN Project Officer:

Peta-Anne Paul-Brent

phone 61731765817

fax 61731765663

University of Queensland, School of Medicine

Ground Level, Building 33, Princess Alexandra Hospital

Ipswich Rd, Woolloongabba Queensland 4102

AKTN Protocol - FAVOURED Study

Version 9 30/11/2010 
Table of contents

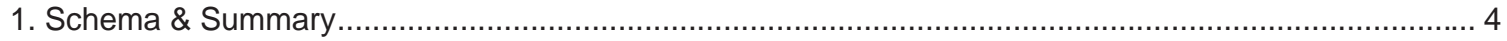

1.1 Schema

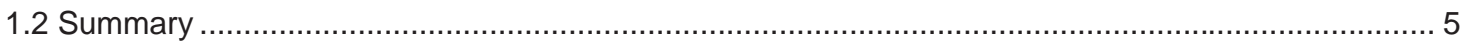

2. Objectives

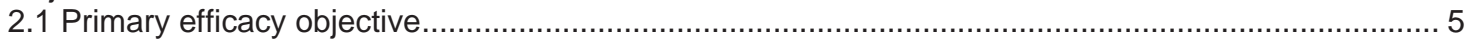

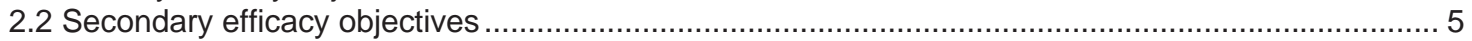

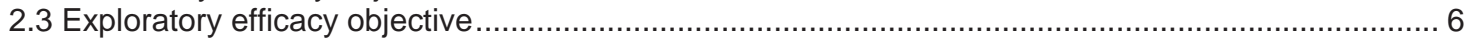

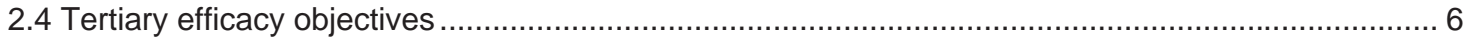

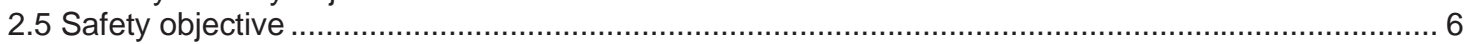

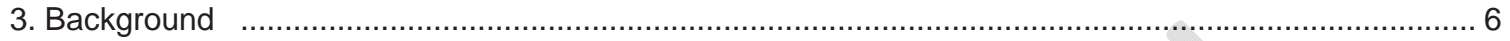

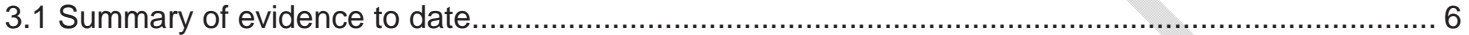

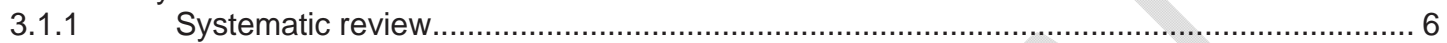

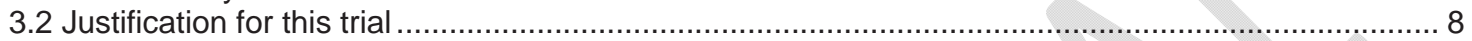

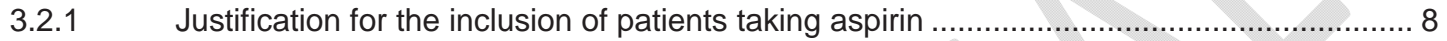

3.2.2 Justification for the amendment of the study outcomes .......................................... 9

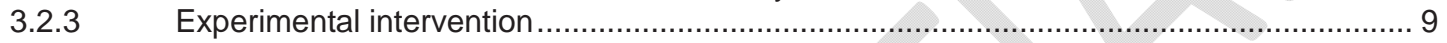

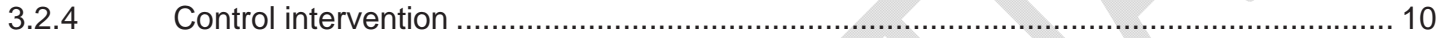

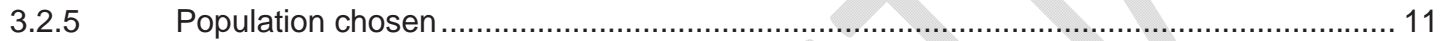

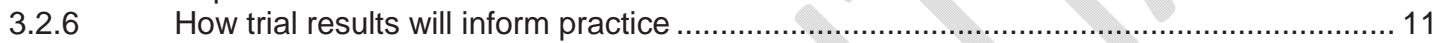

4. Trial Design

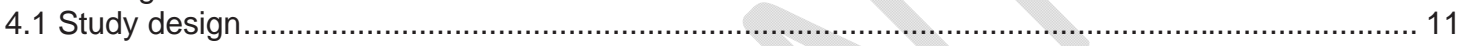

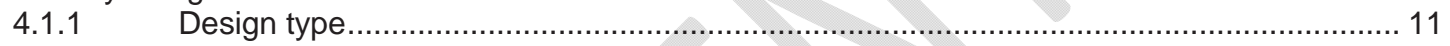

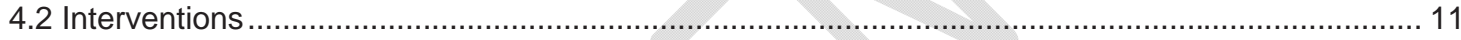

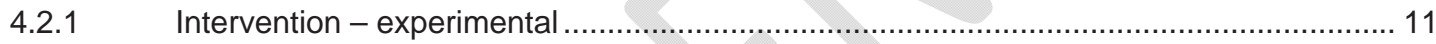

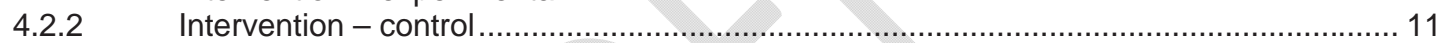

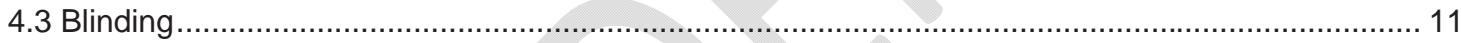

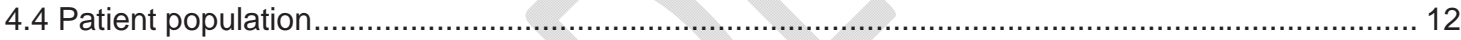

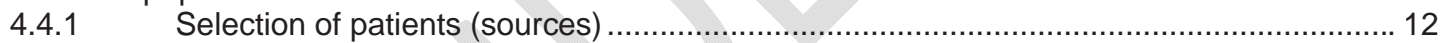

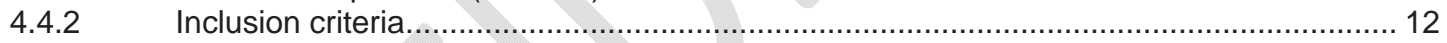

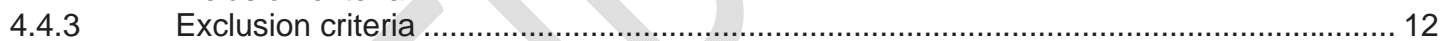

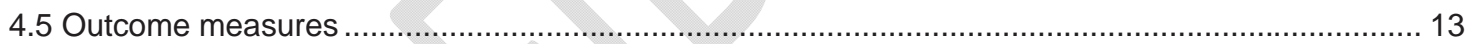

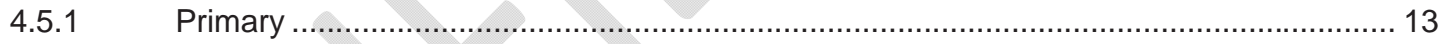

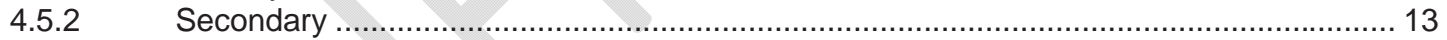

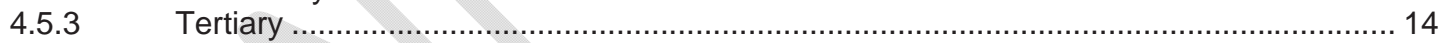

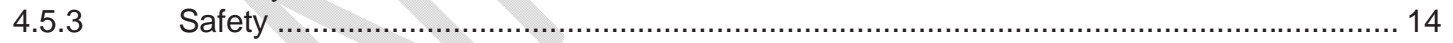

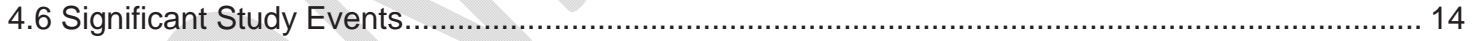

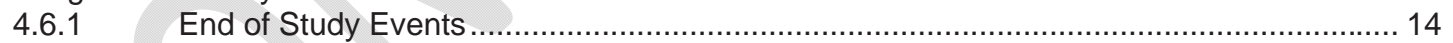

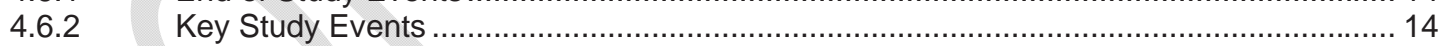

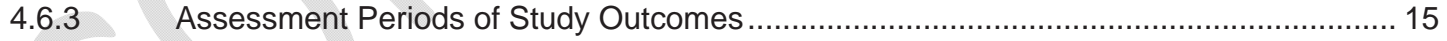

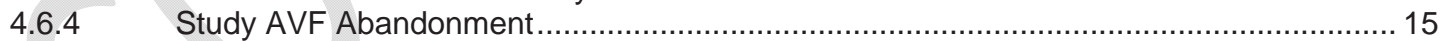

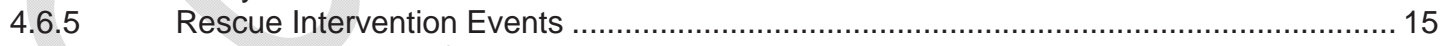

4.6.6 Patient withdrawal from study medication ......................................................... 16

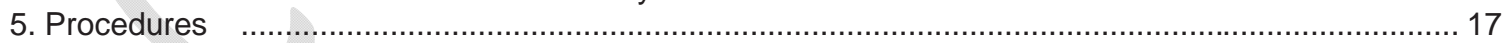

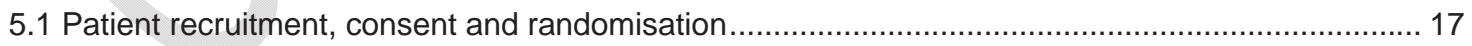

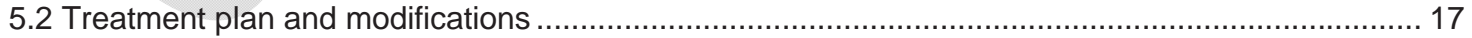

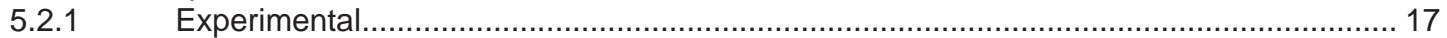

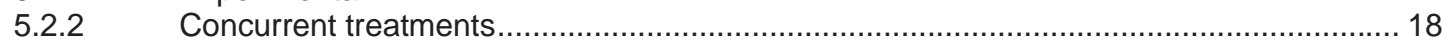

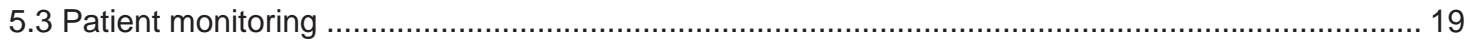

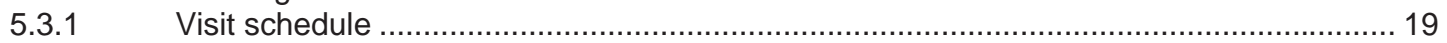

Clinical assessment of outcome

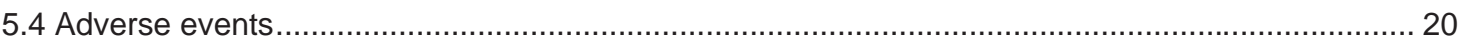

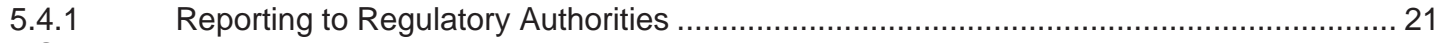

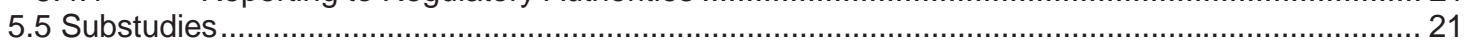

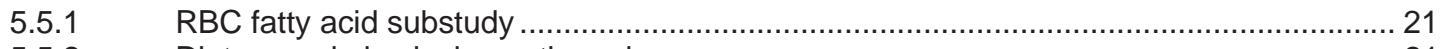

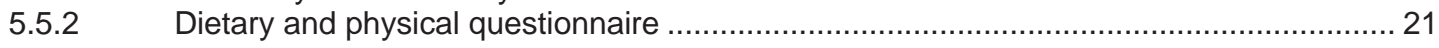

AKTN Protocol - FAVOURED Study

Version 9 30/11/2010

Page 2 of 50 


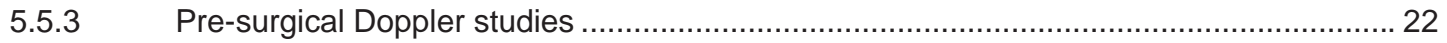

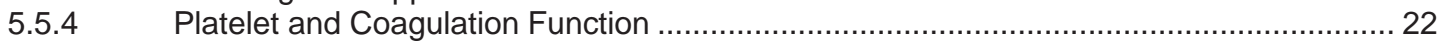

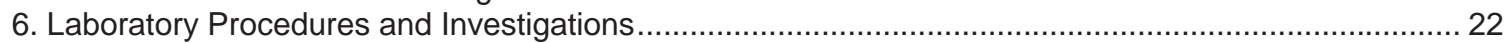

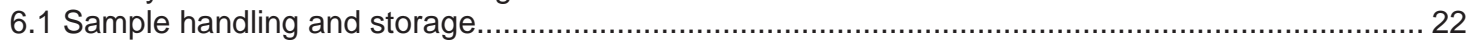

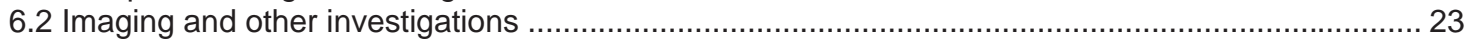

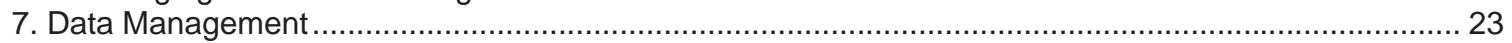

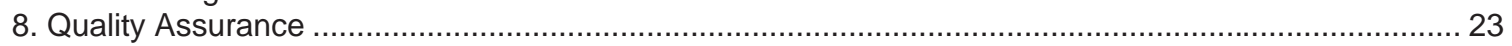

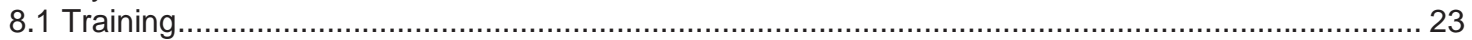

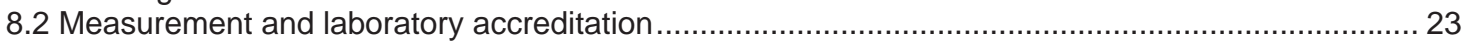

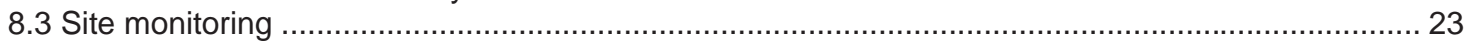

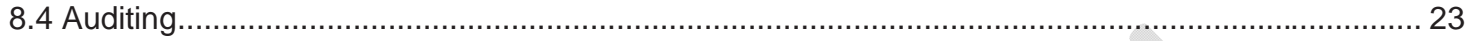

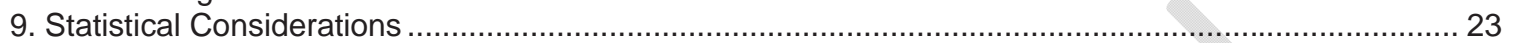

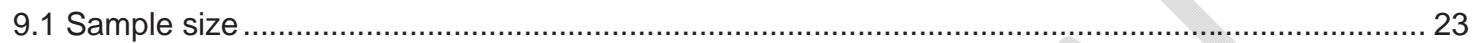

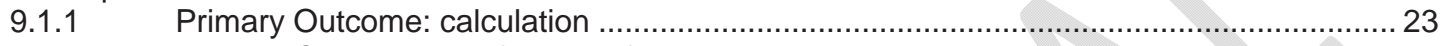

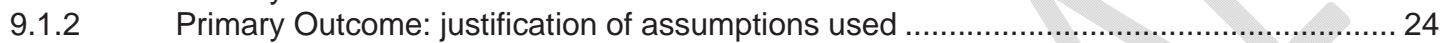

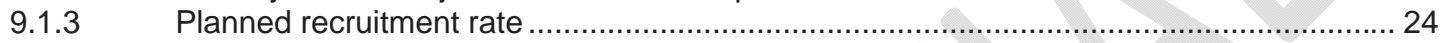

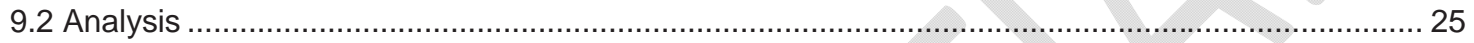

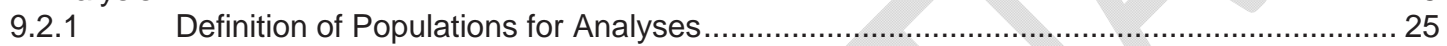

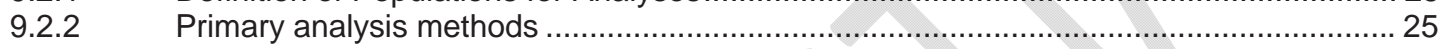

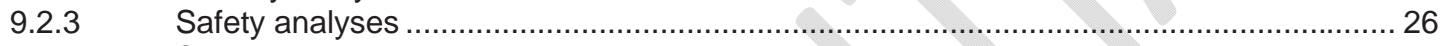

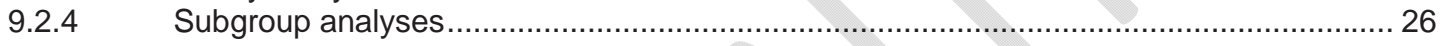

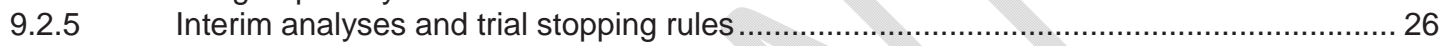

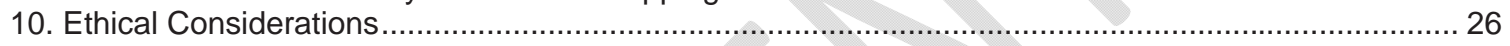

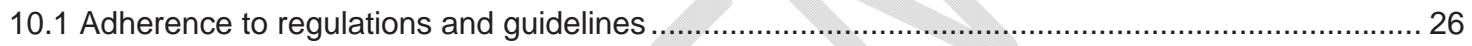

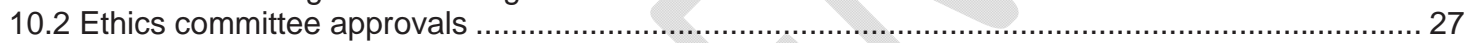

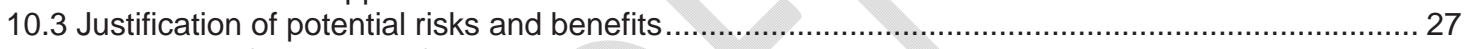

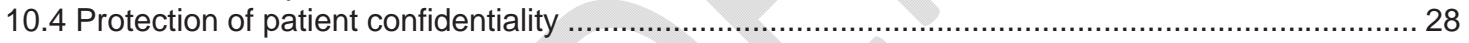

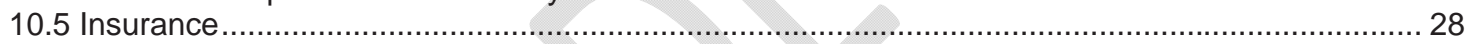

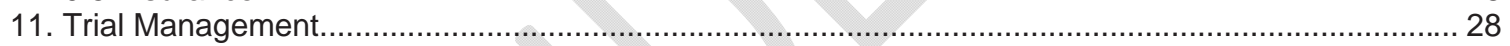

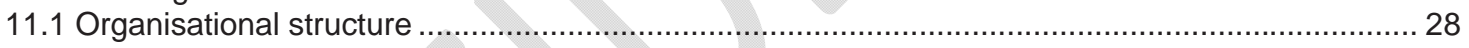

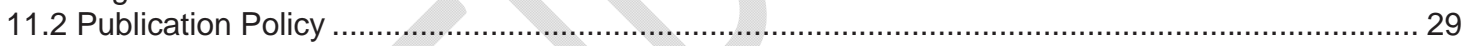

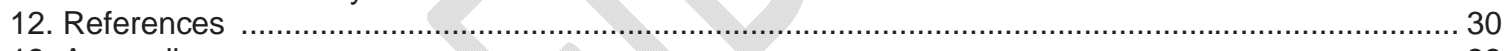

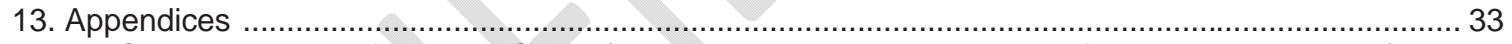

13.1 Sample Patient Information Sheet (Patients randomised to omega-3 fatty acids and aspirin)...... 33

13.2 Sample Patient Information Sheet (Patients randomised to omega-3 fatty acids only) ................. 37

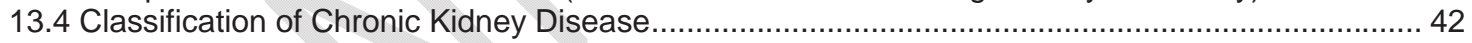

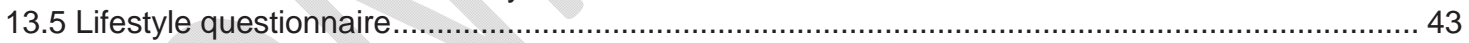

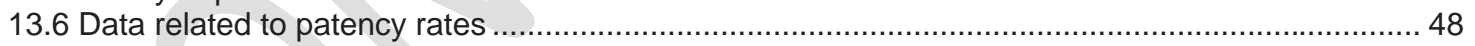




\section{Schema \& Summary}

\subsection{Schema}

Eligible Patients

Key Inclusion Criterion: Stage 4/5 CKD patients undergoing or planning HD with planned de novo AVF

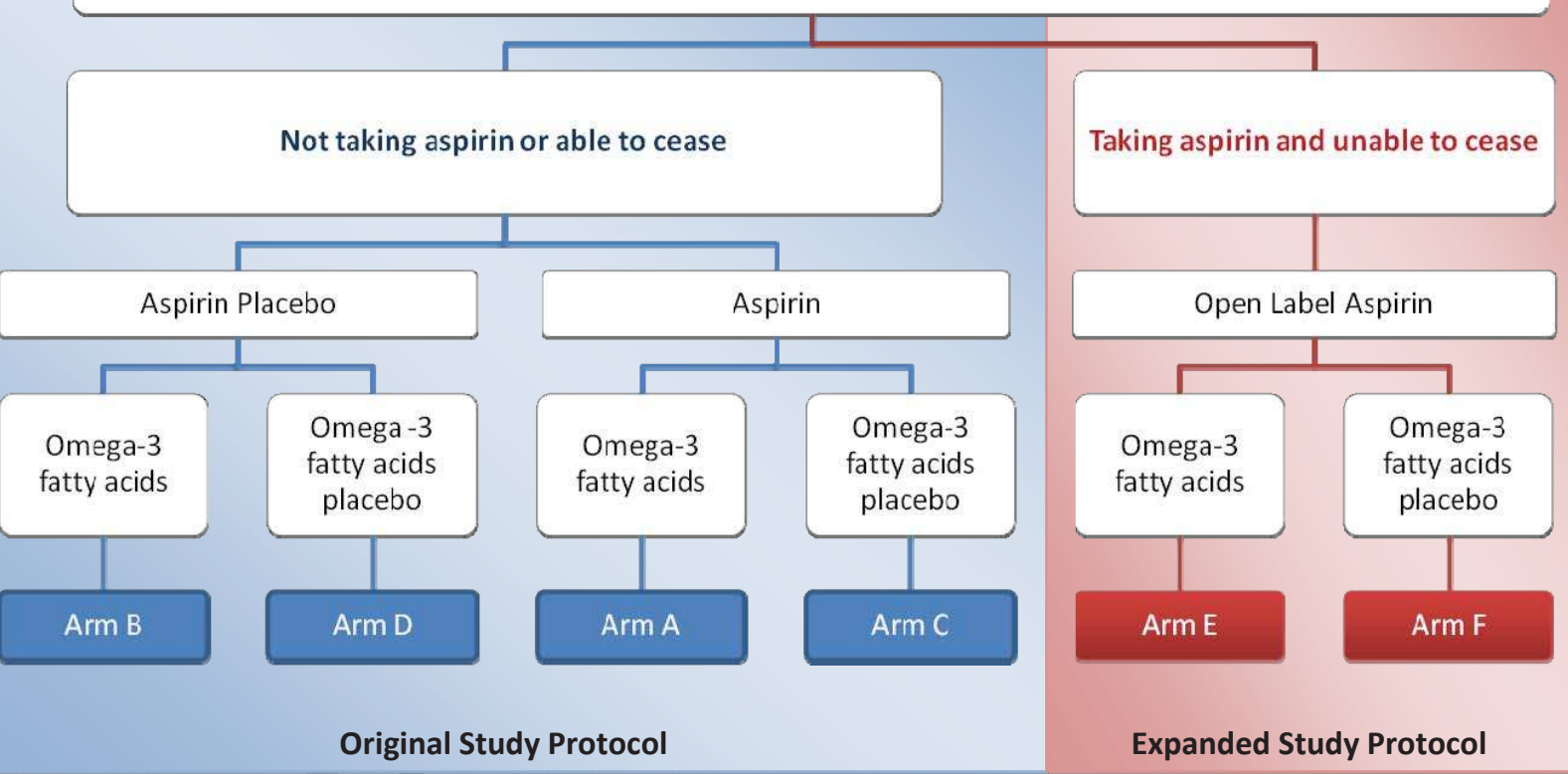

AKTN Protocol - FAVOURED Study 


\subsection{Summary}

FAVOURED is a multicentre, randomised controlled trial with two-by-two factorial design. The objectives of this trial are to determine whether the use of the omega- 3 fatty acids and to a lesser extent, aspirin, will effectively improve postsurgical outcomes for patients with de novo arterio-venous fistulae (AVF).

The study population are patients with stage IV or V chronic kidney disease (CKD) who require or will require haemodialysis (HD) and who are scheduled to undergo creation of an AVF. The primary outcome is AVF Access Failure, which is a composite of Thrombosis, AVF Abandonment, and Cannulation Failure during the Cannulation Assessment Period (CAP). Secondary outcomes include AVF access failure according to strata of aspirin use, safety and adverse events of omega-3 fatty acids and aspirin alone or in combination, catheter use, and rescue interventions.

For the purposes of this protocol "omega- 3 fatty acids" refers to the two marine-derived long-chain polyunsaturated fatty acids, eicosapentaenoic acid (EPA) and docosahexaenoic acid (DHA).

\section{Objectives}

\subsection{Primary efficacy objective}

The primary objective of this trial is to determine whether the use of omega- 3 fatty acids reduce the AVF Access Failure rate of de-novo arterio-venous fistulae (AVF), compared to placebo within 12 months post AVF surgery.

\subsection{Secondary efficacy objectives}

To study the effect of omega-3 fatty acids compared placebo on the following categories of objectives:

\section{Thrombosis}

a. the rate of AVF Thrombosis within 12 months post AVF surgery

b. the rate of primary patency at various time points after surgery (within 24 hours, at weeks 1, 6 and 12 and months 6 and 12)

c. the rate of rescue interventions within 12 months post AVF surgery

d. time to the first rescue intervention

2. Permanent AVF abandonment

a. the rate of permanent abandonment within 12 months post AVF surgery

b. time to permanent abandonment of the study AVF

3. Cannulation

a. the rate of Cannulation Failure during the Cannulation Assessment Period

b. time to the first successful cannulation 


\section{Central Venous Catheter (CVC) requirement}

a. for haemodialysis on $\geq 1$ occasion between 12 weeks and 12 months post AVF Surgery

b. for haemodialysis on $\geq 1$ occasion during Cannulation Assessment Period

c. for haemodialysis on $\geq 1$ occasion from end of Cannulation Assessment Period until 12 months post AVF Surgery

d. the number of days a CVC is present in situ between 12 weeks and 12 months post AVF Surgery

\subsection{Exploratory efficacy objective}

To examine whether the use of aspirin reduces AVF Access Failure rate, compared to placebo at 12 months post AVF surgery and possibly on the secondary endpoints listed above.

\subsection{Tertiary efficacy objectives}

1. To determine whether aspirin and/or omega-3 fatty acids, compared with placebo, reduce the number of days a CVC is present in situ within 12 months post AVF surgery.

2. To study the natural history and long term outcome (beyond 12 months) of the de-novo AV fistulae

\subsection{Safety objective}

To compare adverse event rates, particularly bleeding, for omega-3 fatty acids and aspirin alone and in combination, compared to placebo.

\section{Background}

\subsection{Summary of evidence to date}

\subsubsection{Systematic review}

Severe chronic kidney disease (CKD) is increasing in both incident and prevalent cases due to ageing of the population and co-morbid conditions such as diabetes. Haemodialysis (HD) is utilised by $70 \%$ of patients with end-stage kidney disease (ESKD) in Australia. However, effective haemodialysis is critically dependent upon obtaining and maintaining repeated access to the circulation. Delivering the required blood flow ( $>300 \mathrm{ml} /$ minute) necessary has long been referred to as the "Achilles' heel" of haemodialysis. Vascular access options include native arterio-venous fistula (AVF), synthetic arterio-venous graft (AVG) or central venous catheter (CVC). AVF is universally acknowledged as the optimal access device with the best long-term patency, lowest cost and lowest infection rates [1-3] and is the most prevalent access used in Australasia (75\%) [4]. Because vascular access related surgical procedures and complications of vascular access represent a high proportion of all admissions in the ESKD population and are a major economic burden for health care providers [5], various international initiatives have made this a focus of quality care improvements [6]. 
Complications associated with artificial vascular access devices (AVG and CVC) include sepsis (especially staphylococcal), vascular malfunction (thrombosis) and death. Several studies confirm the risk (Hazard Ratio) of all cause death is (using AVF as the reference group) around 1.5 for AVG and 3.0 for CVC with similar rates for infectious mortality [2, 3, 7].

While the clinical imperative is to establish AVF in as many patients as possible, primary failure due to thrombosis and failure of mature are major impediments to clinical success [8]. Reports in the literature from the 1970s and 1980s generally describe primary failure rates of between $10-20 \%$. More contemporary publications (1990's on) are less encouraging with reports of the primary failure rates increasing to between $20-54 \%$. The increase of primary failure in the more recent era may reflect changing patient factors, such as older patients and a higher prevalence of diabetes. A meta-analysis examining the primary failure rate of AVF [9] found a pooled estimated primary failure rate of $15.3 \%$. However, this meta-analysis included early studies starting in the $1960 \mathrm{~s}$ and omitted a number of key studies published after 2002. These more recent studies have a higher primary failure rate $(20-54 \%$ in studies published since 1999) [1]. Consistent with these findings of high early failure rate, $50 \%$ of new patients commence haemodialysis in Australia and New Zealand with a CVC, due to a failed or inadequate AVF [10].

Primary failure usually occurs as a result of one of two processes [8]:

1) Thrombosis, which usually occurs within weeks of the procedure; and

2) Inadequate size of the artery or maturation of the vein.

Strategies to reduce primary failure rates include pre-operative identification of unsuitable anatomy by the use of ultrasound, improved surgical technique and pharmacological interventions designed to prevent vessel occlusion (thrombosis).

Early thrombosis is defined as thrombosis within the first 30 days post-operatively [11]. There have been a number of small trials evaluating pharmacological agents aimed at reducing the early thrombosis rates. These trials utilised the anti-platelet agents: aspirin, sulphinpyrazone and ticlopidine and varied in size from as few as 5 patients up to 261 patients. In one study using ticlopidine, fistula thrombosis rates for the ticlopidine versus placebo groups were $12 \%$ versus $19 \%(p=0.10)$, respectively. Pooled data for ticlopidine suggests a reduction in thrombosis rate from $25 \%$ to $12 \%(p<0.001)$ [12]. Studies using sulphinpyrazone showed variable results, but were underpowered with the largest study enrolling only 36 patient [13]. Andrassey et al $(n=92)$ compared patients given $500 \mathrm{mg}$ aspirin daily for 4 weeks with placebo; the thrombosis rate in the aspirin group was $4 \%$ compared with the placebo group of $24 \%$, OR $0.15(0.03,0.73)$ [14]. Another study [15] with a smaller sample size $(n=68)$, did not demonstrate a benefit from aspirin.

Studies examining the effect of omega-3 fatty acids on vascular access are not common. There has only been one study exploring the efficacy of omega-3 fatty acids in the prevention of vascular access thrombosis. This study involved the use of omega-3 fatty acids as a single agent and explored its use in AVG, not AVF. Importantly, the study demonstrated a dramatic reduction in thrombosis at 1 year in patients with AVG [16]. There has also been a study that examined the effect of low-dose aspirin in combination with omega-3 fatty acids on whole blood eicosanoid production [17]. This demonstrated an additive effect of the combination on thromboxane $\mathrm{A}_{2}\left(\mathrm{TxA}_{2}\right)(40 \%$ with aspirin alone vs. $62 \%$ with the combination) and a smaller reduction in the concentrations of prostacyclins $\left(\mathrm{PGl}_{2}\right.$ and $\left.\mathrm{PGl}_{3}\right)$ compared with aspirin alone. There are other effects of omega-3 fatty acids which may be particularly beneficial in patients with CKD, including

AKTN Protocol - FAVOURED Study

Version 9 30/11/2010

Page 7 of 50 
improvement in lipid profile [16, 18, 19], blood pressure and heart rate reduction [20], and attenuated inflammatory responses [21] and oxidative stress [22]. Other postulated but as yet unproven benefits in the CKD population may include a reduction cardiovascular mortality and a reduction in uraemic pruritis [23].

\subsection{Justification for this trial}

The failure of vascular access is the most common cause for hospital admission in CKD patients. Although there is some data to suggest that anti-platelet agents may increase the primary patency of AVF, the limited evidence base and the uncertainty regarding the choice of agent has not supported the widespread use of anti-platelet agents in the prevention of AVF thrombosis. The number of patients requiring dialysis is growing and the maintenance of vascular access in these patients is one of the major challenges faced by health workers in this area, both in terms of clinical input required and economic burden. Furthermore, studies on the natural history of vascular access outcomes show an increase in the incidence of primary failure, which is most likely related to the changing demographics of patients with an increasing prevalence of older patients and diabetic status. Moreover, there has never been an adequately powered study examining the efficacy of anti-platelet agents in the prevention of AVF Access Failure.

\subsubsection{Justification for the inclusion of patients taking aspirin}

At almost two years into the study, patient recruitment in the FAVOURED trial has been much lower than expected. As of June 2010, 154 patients have been randomised with the recruitment rate about one-ninth of expected. Analysis of screening logs showed that there was a high rate of screening failure. Out of 1,619 patients screened for possible recruitment, 1,180 (73\%) were excluded (remaining 323 patients were either suitable but not yet enrolled or their suitability is unknown). The commonest cause of exclusion from the trial was current use of aspirin in $37 \%$ patients (433 out of 1,180 patients). Data on the indications for aspirin use is not collected. Our repeated attempts over a 12 month period of educating the participating centres on the lack of evidence about benefits of aspirin for secondary prevention failed to improve the recruitment rate. In a DOPPS report published by Ethier et al, aspirin usage in patients on haemodialysis in Australia and New Zealand was $41 \%$ [24]. These data strongly support our findings that aspirin usage is a major barrier to recruitment. It is expected that allowing the inclusion of patients on aspirin will significantly boost the recruitment rate.

Analysis of baseline characteristics of the recruited patients showed that the current study population was young and healthy (with a mean age 55 years and an ischaemic heart disease rate of $4 \%$ ). Since the current study population does not represent contemporary CKD/ESRD population, there is a possibility of weakening the external validity of the study. Previous observational studies have shown that dialysis patients on aspirin were likely to be older with significant cardiovascular disease [24-26]. Thus, with the expanded randomisation process, the study population is more likely to represent the contemporary dialysis population.

A blinded analysis of primary outcome showed much lower primary patency failure rate at 12 weeks than expected (5\% vs. $25 \%$ ). A possible explanation for such a low failure rate could be the relatively young and healthy population as outlined above. Since only 88 patients were included in this analysis, it would be premature to conclude that the study may be under-powered due to very low event rates. Still, if this trend persists, a possibility of an under-powered study cannot be ruled out. The observational studies 
have showed patients taking aspirin were more likely to experience a vascular event possibly due to increased age and cardiovascular burden [24, 26]. It is expected that with the amended study design, the event rates (and hence the study power) will increase with the possibility of recruiting older patients with greater cardiovascular burden.

\subsubsection{Justification for the amendment of the study outcomes}

The FAVOURED trial was designed before the pivotal study examining the effect of Clopidogrel (an anti-platelet agent) in AVF was published [27]. In this study, a total of 877 patients were randomly assigned to clopidogrel or placebo for 6 weeks. Clopidogrel reduced the frequency of early thrombosis of new AV fistula $(12.2 \%$ vs. $19.5 \%$, relative risk $0.63 ; 95 \%$ confidence interval 0.46 to 0.97 ). Despite this, failure to attain suitability for dialysis did not differ between the clopidogrel and placebo groups (61.8\% vs. $59.5 \%$, respectively; relative risk, $1.05 ; 95 \%$ confidence interval, 0.94 to 1.17); Thus demonstrating that 'thrombosis' is not a valid surrogate for usability or suitability of AV fistula. The FAVOURED Trial Management Committee decided that the primary outcome should be amended to a more clinically relevant outcome - AVF Access Failure, which is a composite of Thrombosis, AVF Abandonment and Cannulation Failure (the inability to cannulate the AVF during a specified period after AVF surgery).

\subsubsection{Experimental intervention}

Aspirin has been chosen as one of the interventions in this study because of its wellestablished anti-platelet effects, the trend to efficacy in the underpowered studies to date and because it is both inexpensive and an agent familiar to clinicians. Its use as an antiplatelet agent is well established in clinical practice for other purposes relating to thrombosis prevention particularly in patients with established cardiovascular disease. The anti-platelet effect of aspirin is mediated by its ability to inhibit the platelet cyclooxygenase enzyme resulting in blockade of the synthesis of the pro-aggregatory vasoconstrictor, thromboxane $\mathrm{A}_{2}\left(\mathrm{TxA}_{2}\right)$.

Omega-3 fatty acids have a number of biological effects which make it an attractive agent for the prevention of vascular access thrombosis, including the inhibition of platelet aggregation, anti-inflammatory effects and anti-proliferative actions. Omega-3 fatty acids and aspirin both affect the balance between the pro-aggregatory and vasoconstrictor effects of $\mathrm{TxA}_{2}$ and the anti-aggregatory and vasodilator effects of prostacyclin $\left(\mathrm{PGl}_{2}\right)$ but the mechanism of their effect is different. Omega-3 fatty acids have a weaker inhibitory effect on $\mathrm{TxA}_{2}$ level, its effect mediated by reducing the availability of arachidonic acid (AA), a precursor of $\mathrm{TxA}_{2}$. In addition, omega-3 fatty acids lead to an increase in $\mathrm{PGI}_{3}$ formation (anti-aggregatory and vasodilatation effects equipotent to $\mathrm{PGI}_{2}$ ) [17]. The antiinflammatory effect of omega-3 fatty acids is mediated via a reduction in leukotriene and cytokine production. Theoretically then, a combination of aspirin and omega-3 fatty acids should result in a more favourable effect on platelet aggregation than either agent used alone.

The choice of $100 \mathrm{mg}$ aspirin for patients randomised to aspirin is based on this dose having adequate anti-platelet aggregation properties and is the dose most commonly used in patients with cardiovascular disease. The dose of omega- 3 fatty acids ( $4 \mathrm{~g}$ daily) has been shown to be well tolerated and has benefited multiple cardiovascular risk factors in patients at increased risk of cardiovascular disease [28-32]. The omega-3 fatty acid capsules chosen (Omacor(C), are commercially available and provide the highest concentration of omega-3 fatty acids per gram of oil. We have chosen an oral route of administration for both aspirin and omega-3 fatty acids in this study. The oral absorption of both agents is excellent and thus alternative routes of administration are not required.

AKTN Protocol - FAVOURED Study

Version 9 30/11/2010

Page 9 of 50 
Bleeding episodes are a concern for clinicians in relation to the use of aspirin in this cohort. Even in the general population on chronic low dose aspirin, the risk of serious gastrointestinal bleeding is doubled [33] and the theoretical risk is higher in patients with CKD because of the presence of impaired haemostasis from multiple mechanisms. There have been published studies [34, 35], showing a significant elevation in bleeding times in patients on haemodialysis treatments administered a single dose of aspirin, but surprisingly little evidence-based clinical trial data in this population. This has been explored in the UK-HARP-I study. In this $2 \times 2$ factorial design study involving simvastatin and aspirin as active therapies, allocation to the aspirin group was not associated with an excess of major bleeds (2\% in patients on aspirin vs. $3 \%$ in patients not on aspirin), but there was a three-fold increase in minor bleeding [36]. Importantly though, in this study, patients received aspirin for 12 months, whereas our study has a much shorter period on therapy of 3 months. Exploring the potential risk of even short-term aspirin is a major secondary outcome of this study.

Omega-3 fatty acids appear to be well tolerated, even in high doses, with gastrointestinal complaints particularly nausea, vomiting, diarrhoea and non-specific discomfort being the most often reported. In studies looking at the effect of omega-3 fatty acids on bleeding time it has not been found to be significantly prolonged [37]. In studies that have been done in a CKD cohort, there have been no clear effects demonstrated in relation to platelet aggregation and bleeding times. There has only been one report of a serious bleeding event in a single patient in an uncontrolled study [38]. Furthermore, it has been reported that administration of omega-3 fatty acids can protect the gastric mucosa against aspirin-induced injury, the postulated mechanism being that omega-3 fatty acids counteract the effect of aspirin on the decrease in prostacyclin (combined effect of $\mathrm{PGI}_{2}$ and $\left.\mathrm{PGI}_{3}\right)[39,40]$. Thus the combination of aspirin and omega-3 fatty acids may be expected to be additive in terms of efficacy in vascular access thrombosis, but to have a lower risk of bleeding complications than the use of aspirin alone.

The design of a $2 \times 2$ factorial study is based on the hypothesis that aspirin and omega- 3 fatty acids will both be effective therapies and that the combination of aspirin and omega3 fatty acids will be additive, but not synergistic. In relation to safety concerns, although both aspirin and omega- 3 fatty acids prolong bleeding time, the combination may be expected to be safer than aspirin used alone.

The modified protocol will allow inclusion of patients who are already on aspirin at the time of enrolment and not able to cease treatment. This protocol amendment will result in the majority of patients being randomised to arms $E$ and $F$ (approximately $75 \%$ of the remaining recruitment target will be randomised to omega-3 fatty acids or placebo) and fewer patients (approximately $25 \%$ of the remaining recruitment target) will be randomised to the arms $\mathrm{A}, \mathrm{B}, \mathrm{C}$ and $\mathrm{D}$ (omega-3 fatty acids and aspirin or placebo arms - Please refer to section 1.1 for study schema). The resultant sample size will have adequate statistical power to test the omega-3 fatty acids hypothesis, but not the aspirin hypothesis. Hence, the primary objective is restricted to the omega-3 fatty acids hypothesis and the aspirin hypothesis, is downgraded to a secondary objective. Despite of inadequate statistical power, recruitment of eligible patients to the arms $A, B, C$ and $D$ will be continued to obtain the following crucial data: (1) adverse effects of omega- 3 fatty acids alone or in combination with aspirin, (2) adverse effects of aspirin.

\subsubsection{Control intervention}

Currently patients are not given any prophylactic agents in the majority of cases. This is because the studies to date using anti-platelet agents for secondary prevention have not 
been definitive in demonstrating an effect, and concern exists about the safety of the agents in respect to bleeding events. Therefore use of placebo is appropriate.

\subsubsection{Population chosen}

The study will be multi-centre, and open to all public and private hospital renal units that perform vascular access procedures and will be conducted in Australia and selected overseas countries, namely New Zealand, the United Kingdom, and Malaysia.

\subsubsection{How trial results will inform practice}

If the trial demonstrates a positive effect of either or both agents, this will lead to a reduction in thromboses, quicker time to dialysis access, and a need for less revisional surgery.

If the trial demonstrates no effect of the agents, this will suggest that platelet aggregation is not the major mechanism for thrombosis in this population and that it may be the poor status of vessels used to create the anastomosis that is a more important factor.

\section{Trial Design}

\subsection{Study design}

\subsubsection{Design type}

The original protocol is a $2 \times 2$ factorial-design trial, where patients are randomised to aspirin or matching placebo, and also to omega-3 fatty acids or matching placebo, resulting in four treatment groups. Randomisation will be achieved using a minimization method, balancing over the two stratification factors of 1) study site and 2) upper versus lower arm AVF.

Patients who are currently not taking aspirin or those taking aspirin but are able to cease treatment for the purpose of entering into the trial will be randomised as above. Suitable patients who are on aspirin at the time of screening and are unable to cease, will proceed with the expanded protocol, in which they will be allowed to continue with open-label aspirin and randomised to omega-3 fatty acids or matched placebo only.

\subsection{Interventions}

\subsubsection{Intervention - experimental}

The principal action of both aspirin and omega- 3 fatty acids is by their ability to reduce platelet aggregation. Omega-3 fatty acids have other potentially beneficial effects on rheology, inflammation and blood flow.

\subsubsection{Intervention - control}

Matching placebo for each agent.

\subsection{Blinding}

Each of the two interventions will be studied using a matching placebo, in order to make this a double-blind trial. At the completion of treatment, patients and investigators will be asked which treatment they believe the patient has been receiving, in order to have a measure of the degree of effectiveness of blinding.

In addition, the primary outcome of AVF Access Failure will be made by an independent observer, unaware of the patient's treatment assignment. 


\subsection{Patient population}

\subsubsection{Selection of patients (sources)}

Patients will be recruited through renal units. The study will be multi-centre, and conducted in Australia and selected overseas countries, namely New Zealand, the United Kingdom, and Malaysia. Public hospital renal unit haemodialysis patients are likely to represent the full population of haemodialysis patients well, as most such patients are treated in these units.

\subsubsection{Inclusion criteria}

Patients must meet the following inclusion criteria:

1. Stage 4 or 5 Chronic Kidney Disease

2. Currently on haemodialysis or haemodialysis is planned to start within 12 months (including patients currently on peritoneal dialysis).

3. Planned AVF will be the primary haemodialysis access mechanism.

4. Surgery to create an arterio-venous fistula in the upper or lower arm is planned.

5. Aged over 19 years

6. Treating team agreeable to patient's involvement in the trial

7. Informed consent

\subsubsection{Exclusion criteria}

Patients must not meet any of the following exclusion criteria:

1. Revision of existing AVF rather than de novo AVF

2. Medical indication for anti-platelet or thrombolytic agents*

3. Known intolerance of agents including hypersensitivity to aspirin, allergy to any other NSAIDs or fish

4. Current use of aspirin within two weeks of commencing trial, or of omega-3 fatty acids within 4 weeks of commencing trial*

5. Pregnancy, lactation or intention to fall pregnant during the time course of the study

6. Known bleeding disorder or established diagnosis of active or suspected bleeding

7. History of Gl ulcers or bleeding within the last 3 months

8. Platelet count less than $100 \times 10^{9} / \mathrm{L}$

9. Known active peptic ulcer disease

10. Severe hepatic insufficiency

11. Already receiving anti-coagulation therapy such as warfarin

12. Receiving regular non-steroidal anti-inflammatory (NSAIDS) agents for another indication such as arthritis

13. Syndrome of asthma, rhinitis and nasal polyps if uncontrolled on usual therapy

14. Plan to have other (non-access) surgery within 2 weeks of trial medication period where, in the opinion of the investigator, aspirin or omega-3 fatty acids would be contraindicated for the planned procedure.

15. Potential non-compliance with treatment regimen in the view of the treating clinicians

16. Involved in another clinical trial where the intervention being trialled is likely to confound the outcome of this trial

17. Previously randomised to this trial.

*Note: Patients who fail to meet the exclusion criteria 2 (Medical indication for antiplatelet or thrombolytic agents) and exclusion criteria 4 (Current use of aspirin within two weeks of commencing trial), and who are otherwise suitable will be enrolled into the expanded protocol. 


\subsection{Outcome measures}

\subsubsection{Primary}

AVF Access Failure is a composite of

- Thrombosis: This is defined as the absence of a thrill or bruit clinically and/or the requirement of Rescue Intervention to restore patency for thrombosis or occlusion for the study AVF between AVF Surgery and Month 12 Visit. Please refer to section 4.6.5 for further information about Rescue Intervention events.

- AVF Abandonment: This is defined as the permanent abandonment of study AVF between AVF Surgery and Month 12 Visit. Please refer to section 4.6.4 for further information about AVF abandonment.

- Cannulation Failure: This is defined as the failure to successfully cannulate the study AVF with 2 needles (or with 1 needle if using single needle dialysis method) during 8 or more $12 \mathrm{HD}$ sessions. Please refer to Section 4.6.3 for further information about Assessment Periods.

\subsubsection{Secondary}

\section{Thrombosis}

- Thrombosis: This is defined as the absence of a thrill or bruit clinically and/or the requirement of Rescue Intervention to restore patency for thrombosis or occlusion for the study AVF between AVF Surgery and Month 12 Visit. Please refer to section 4.6.5 for further information about Rescue Intervention events.

- Primary patency at various time points: This is defined as the presence of an audible bruit over the site of the arterio-venous anastomosis at time points of within 24 hours post surgery (Recovery visit), and Visits Weeks 1, 6 and 12 and Months 6 and 12.

- Number and type of Interventions: This is defined as the number and type of interventions (both rescue and non-rescue) the study AVF requires between AVF Surgery and Month 12 Visit. Please refer to section 4.6.5 for further information about Rescue Intervention events.

- Time to first Rescue Intervention: This is defined as the time from AVF creation to first occasion of rescue intervention up to Month 12 Visit. Please refer to section 4.6.5 for details of the Rescue Intervention events.

Permanent AVF abandonment

- $\quad$ AVF Abandonment: This is defined as the permanent abandonment of study AVF between AVF Surgery and Month 12 Visit. Please refer to section 4.6.4 for further information about AVF abandonment.

- Time to AVF Abandonment: This is defined as the time from AVF Surgery to permanent abandonment of study AVF up to Month 12 Visit. Please refer to section 4.6.4 for further information about AVF abandonment

\section{Cannulation}

- Cannulation Failure: This is defined as the failure to successfully cannulate the study AVF with 2 needles (or with 1 needle if using single needle dialysis method) during 8 or more 12 HD sessions. Please refer Section 4.6.3 for the Cannulation Assessment Periods.

- Time to the first Successful Cannulation: This is defined as the time taken from the AVF Surgery until the first successful attempt at access cannulation up until Month 12 Visit. 


\section{Central Venous Catheter (CVC) requirement}

- $\quad$ CVC Requirement between Visits Week 12 and Month 12: This is defined as the use of a CVC on any occasion to provide vascular access for HD between Week 12 and Month 12 Visits.

- $\quad$ CVC Requirement during CAP: This is defined as the use of a CVC on any occasion to provide vascular access for HD during Cannulation Assessment Period. Please refer to section 4.6.3 for further information about assessment periods.

- CVC Requirement after CAP: This is defined as the use of a CVC on any occasion to provide vascular access for HD after Cannulation Assessment Period to Month 12 Visit. Please refer to section 4.6.3 for further information about Assessment Periods.

- Days of CVC: This is defined as the number of days a CVC is present in situ between Week 12 and Month 12 Visits.

\subsubsection{Tertiary}

- CVC Requirement at any occasion: This is defined as the use of a CVC on any occasion to provide vascular access for HD between AVF Surgery and Month 12 visit.

- Long term outcome of AVF: This is defined as the rate of permanent abandonment at Month 24 and 36 Visits and the time to permanent abandonment up to Month 36 Visit.

\subsubsection{Safety}

- Adverse events: All serious adverse events and adverse drug reactions (see section 5.4) will be collected up to Month 6 Visit. The analysis of this secondary outcome will focus specifically on bleeding events

\subsection{Significant Study Events}

\subsubsection{End of Study Events}

End of Study Events are events that mean the end of study with no further follow up with participants. End of study events include

- death;

- withdrawal of patient's consent;

- events that indicate that the patient is no longer eligible for the study i.e. patient has AVG inserted at Surgery instead of an AVF;

- patient lost to follow up;

- the end of study (12 months from the last patient's surgery).

The ANZDATA registry patient information may be accessed to confirm end of study events, particularly for patients lost to follow up.

\subsubsection{Key Study Events}

Key Study Events are events that do not mean the end of study for patients but are important to record with relation to study outcome measures and the patients' progress in the study. These events include:

- Recovery of renal function (such that dialysis is no longer needed);

- Transfer to peritoneal dialysis (such that haemodialysis is no longer needed);

- Renal transplant;

- Thrombosis of the study AVF; 
- Use of CVC as HD access;

- Imaging of the study AVF.

\subsubsection{Assessment Periods of Study Outcomes}

Most study outcomes are assessed in the period between AVF surgery and Month 12 Visit, which is the minimum follow up period for study participants.

Variations to this period include:

- Cannulation Failure - The Cannulation Assessment Period (CAP) is based on when the patient commences maintenance haemodialysis. Please refer to Table 1 for the definition of Cannulation Assessment Periods.

Table 1 - Cannulation Assessment Periods (CAPs)

\begin{tabular}{ccc}
\hline Start of Maintenance HD & Beginning of CAP & Duration of CAP \\
\hline Prior to Week 12 Visit & $\begin{array}{c}\text { First HD session } \\
\text { after Week 12 Visit }\end{array}$ & $\begin{array}{c}\text { First } 12 \text { consecutive } \\
\text { HD sessions }\end{array}$ \\
\hline $\begin{array}{c}\text { Between Week 12 and } \\
\text { Month 12 Visits }\end{array}$ & $1^{\text {st }}$ HD session & $\begin{array}{c}\text { First } 12 \text { consecutive } \\
\text { HD sessions }\end{array}$ \\
\hline After Month 12 Visit & No CAP & No CAP
\end{tabular}

- Serious Adverse Events and Adverse Drug Reactions - SAEs and ADRs will be assessed for the 3 months of treatment and for 3 months afterwards (Up until Month 6 Visit).

\subsubsection{Study AVF Abandonment}

Abandonment of the study AVF is defined as the point that which the AVF is unable to be cannulated and no further attempts will be made to rescue or revise the access.

The date that the AVF is abandoned is determined at the discretion of site PI. However, events that may indicate AVF abandonment include:

- thrombosis of study AVF;

- imaging of study AVF that shows that the AVF is unusable and not amenable to any intervention for its improvement;

- insertion of another dialysis access including new AVF, AVG, venous catheter or peritoneal dialysis access;

- ligation of study AVF. If the AVF is ligated due to a reason other than thrombosis i.e. steal syndrome or heart disease, this abandonment will not be included in the primary outcome.

\subsubsection{Rescue Intervention Events}

There are two types of intervention events, Rescue interventions and Non-rescue interventions.

Rescue Interventions in the study are those designed to restore patency (absence of flow demonstrated clinically by absence of bruit/thrill or radiologically by absence of flow) of the AVF and include:

- Medical Thombolysis

- Surgical thrombectomy. 
Non-rescue Interventions are those designed to improve functionality in an otherwise patent AVF and include:

- Surgical or radiological revision or dilatation of the AVF from or proximal to the anastomosis to the ipsilateral central vein.

- Dilation of central venous stenosis.

- Ligation of tributaries.

- Superficialisation of AVF.

- Ligation of fistula or salvage by DRIL (Distal reconstruction and interval Ligation) due to distal ischaemia (steal).

\subsubsection{Patient withdrawal from study medication}

Withdrawal of study medication may be made at the discretion of the site PI following a SAE or key study event. However, subjects should still be followed for the duration of the study where possible, even if they have ceased treatment. This will include attendance at scheduled trial visits, and data collection, particularly of outcomes. The investigator may withdraw a subject from study medication at any time if it is felt to be in the best interest of the subject.

Study treatment may be discontinued if one of the following occurs:

- A major bleeding event (e.g. haemorrhagic stroke, gastro-intestinal bleed);

- The access is abandoned due to thrombosis at any time after the one week assessment;

- Anti platelet therapy or anticoagulation is indicated because of other co morbid events i.e. major cardiovascular event, particularly for patients randomised to original protocol;

- A female patient becomes pregnant while on study medication. The event should be reported to the trial coordinating centre immediately as a medically important $\mathrm{SAE}$. The event will be reported within 24 hours and follow up performed through to delivery;

- Patient receives renal transplant.

Study treatment should be continued if:

- Further access is created (including a new AVF or AVG, peritoneal and venous catheter) because of failure of maturation of the study AVF, i.e. access is still patent;

- Renal function is recovered; the study AVF can still be measured patency.

Table 2: Study medication continuation based on key study event or SAE occurrence

\begin{tabular}{|c|c|}
\hline Study Outcomes & Continue Medication? \\
\hline Renal transplant & No \\
\hline Renal function recovered & Yes $^{*}$ \\
\hline Thrombosis of study AVF & $\begin{array}{c}\text { No } \\
\text { if occurs after } 1 \text { week }\end{array}$ \\
\hline Pregnancy & No \\
\hline New AVF or AVG & Yes* \\
\hline $\begin{array}{c}\text { Peritoneal dialysis } \\
\text { commenced }\end{array}$ & Yes* \\
\hline Venous catheter & Yes* \\
\hline Major bleed & No \\
\hline Anti-platelet therapy & No \\
\hline
\end{tabular}

AKTN Protocol - FAVOURED Study

Version 9 30/11/2010

Page 16 of 50 
Patients withdrawn from treatment will still be regarded as being "on study", and follow up data will be collected, unless the patient withdraws consent.

If a patient withdraws consent for the study they shall be withdrawn from the study. Any patient is free to withdraw their consent at any time without the need to justify their decision.

\section{Procedures}

\subsection{Patient recruitment, consent and randomisation}

Participants will be recruited from renal units providing haemodialysis and vascular access services in Australia and selected overseas countries, namely New Zealand, the United Kingdom, and Malaysia. Patients will meet the inclusion and exclusion criteria and be scheduled to undergo surgery for creation of a de novo AV fistula.

Patient consent forms will be approved by the Human Research Ethics Committee at each participating site prior to the beginning of the trial. A sample consent form and patient information sheet is provided to participating sites. Participating sites will file a copy of the approved consent form and information sheet for their site with the coordinating office (AKTN). After discussing the trial, ample time will be given to the participant, accompanying person or legal representative to inquire about the trial and decide whether to participate. No person involved with the trial will coerce or unduly influence the decision of a patient to participate in a trial. A copy of the signed consent form and the patient information sheet will be supplied to the participant. Patient consent must be obtained prior to the registration or initiation of trial procedures. Patients will not be randomised until a signed consent form is filed at site.

The Trial Management Committee (TMC) will monitor the medical literature, and any other relevant information impacting on the continuation of the trial. Consent forms and patient information sheets will be revised should any relevant and important new information become available.

Patients are to be randomised as close as possible to the time of the scheduled procedure and not more than 7 days before the planned procedure. Patients are to commence taking study medications on the day prior to the scheduled surgery. If the procedure is rescheduled, the patient is to cease taking medications if already started and to recommence the day before the rescheduled surgery. Refer to the operations manual for further information. Stratification will occur for study site and upper vs. lower arm site. Patients will be randomised to one of four treatment groups in equal proportion.

\subsection{Treatment plan and modifications}

\subsubsection{Experimental}

For all patients; Omega-3 fatty acids $4 \mathrm{gm}$ daily in the form of 4 Omacor capsules (46\% eicosapentaenoic acid (EPA) and 38\% docosapentaenoic acid (DHA), as the ethyl esters), or 4 matching placebo capsules (olive oil), supplied by Abbott Products, commencing on the day prior to surgery and continuing for 12 weeks.

For patients not taking open-label aspirin; Aspirin $100 \mathrm{mg}$ per day p.o. or matching placebo, supplied by Bayer Healthcare, commencing on the day prior to scheduled surgery and continuing for 12 weeks. Patients taking aspirin and unable to cease will continue with their current open-label aspirin. Aspirin use and dosage should preferably 
remain unchanged for these patients during the treatment period (12 weeks), and any deviations or changes recorded on the case report forms (CRF).

To improve compliance and minimise gastro-intestinal discomfort, the omega-3 fatty acids or matching placebo medications are to be taken morning and evening with 2 capsules taken with a cold drink just before breakfast and 2 capsules again taken with a cold drink just before dinner.

If patient's access surgery is postponed, patient should stop medication until $24 \mathrm{hrs}$ before rescheduled surgery. If the surgery is postponed for more than 4 weeks, the patient must have another blood test and their eligibility (conformity with the inclusion/exclusion criteria) must be reassessed. If they remain eligible, they should commence the medication which they have already been allocated.

Compliance will be monitored by capsule/tablet count at scheduled study visits at 12 weeks. Australian and New Zealand centres will also collect erythrocyte fatty acid samples for testing for compliance with omega-3 fatty acid intake. All centres will apply routine biochemistry (lipids, CRP) and haematology analyses as part of patient monitoring (refer to section 6.1)

\subsubsection{Concurrent treatments}

No other fish oil/omega-3 fatty acid supplement, or anti-coagulation agents are permitted during the period the patient is taking study medication. Patient randomised into the original protocol will not be permitted to take any other anti-platelet agents and patients randomised to the expanded protocol with only be allowed to take their current openlabel aspirin which should be maintained at a constant dosage level throughout the treatment period. Heparin or heparin-like agents that are used during vascular surgery or during haemodialysis to flush lines are permitted during the study treatment period. Once the treatment period is completed, patient may commence or recommence omega- 3 fatty acids or aspirin product.

In addition, interaction of the following agents with aspirin should be considered:

- Thrombolytic agents

- Methotrexate, particularly at doses of $15 \mathrm{mg} /$ week or greater

- Uricosuric agents

- Digoxin

- Sufonylureas or insulin

- Systemic glucocorticoids, except replacement therapy for Addison's disease

- ACE inhibitors, beta blockers

- Valproic acid, phenytoin

- Acetazolamide

- Diuretics (if daily asa dose is $>3 g$ ) 


\subsection{Patient monitoring}

\subsubsection{Visit schedule}

Table 3: FAVOURED Study Visit Schedule

\begin{tabular}{|c|c|c|c|c|c|c|c|c|c|c|}
\hline Study Phase & Baseline & Randomisation & Surgery & & $\begin{array}{l}\text { atm } \\
\text { od ( }\end{array}$ & & & $\begin{array}{l}\text { ow } \\
\text { (n }\end{array}$ & $\begin{array}{l}\text { p Pe } \\
\text { hs) }\end{array}$ & \\
\hline Assessment & $\begin{array}{c}\leq 4 \text { wks prior } \\
\text { to surgery }\end{array}$ & $\begin{array}{c}\leq 7 \text { Days prior to } \\
\text { surgery }\end{array}$ & & 1 & 6 & 12 & 6 & 12 & 24 & 36 \\
\hline Informed Consent & $\mathrm{X}$ & & & & & & & & & \\
\hline Subject number & & $\mathrm{X}$ & & & & & 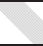 & & & \\
\hline Medical History & $\mathrm{X}$ & & & & & 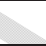 & & 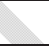 & & \\
\hline Weight and Blood Pressure & $\mathrm{X}$ & & $\mathrm{X}$ & $\mathrm{X}$ & $\mathrm{X}$ & $\mathrm{X}$ & $\mathrm{X}$ & $\mathrm{X}$ & $\mathrm{X}$ & $\mathrm{X}$ \\
\hline Demographics & $\mathrm{X}$ & & & . & & 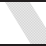 & 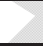 & 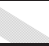 & 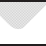 & \\
\hline Physical Exam & $\mathrm{X}$ & & & & t & & 8 & 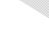 & & \\
\hline Dialysis status & $\mathrm{X}$ & & & $\mathrm{X}$ & $\mathrm{X}$ & $\mathrm{X}$ & $\mathrm{X}$ & $\mathrm{X}$ & $\mathrm{X}$ & $\mathrm{X}$ \\
\hline Description and status of AVF & & & $\mathrm{X}$ & $\mathrm{X}$ & $\mathrm{X}$ & $\mathrm{X}$ & $\mathrm{X}$ & $\mathrm{X}$ & $\mathrm{X}$ & $\mathrm{X}$ \\
\hline AVF Access Failure components & & & $\mathrm{X}$ & $\mathrm{X}$ & $\mathrm{X}$ & $\mathrm{X}$ & $\mathrm{X}$ & $\mathrm{X}$ & & \\
\hline Concomitant meds & $\mathrm{X}$ & & $\mathrm{X}$ & $\mathrm{X}$ & $\mathrm{X}$ & $\mathrm{X}$ & $\mathrm{X}$ & & & \\
\hline Adverse events & & & $\mathrm{X}$ & $\mathrm{X}$ & $\mathrm{X}$ & $\mathrm{X}$ & $\mathrm{X}$ & & & \\
\hline Fasting Pathology Tests & & & & & & & & & & \\
\hline Haematology and coagulation & $\mathrm{X}$ & & & & & $\mathrm{X}$ & & & & \\
\hline Biochemistry and lipid profile & $\mathrm{X}$ & & & & & $\mathrm{X}$ & & & & \\
\hline Urinary protein:creatinine ratio & $\mathrm{X}$ & & 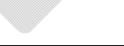 & & & $\mathrm{X}$ & & & & \\
\hline Optional: Homocysteine and Lp(a) & $\mathrm{X}$ & & & & & $\mathrm{X}$ & & & & \\
\hline Stored Samples for ANZ sites (sen & central lab & atory) & & & & & & & & \\
\hline RBC fatty acid sample, hsCRP & $\mathrm{X}$ & & & & $\mathrm{X}$ & $\mathrm{X}$ & & & & \\
\hline $\begin{array}{l}\text { Urinary thromboxane B2 and } \\
\text { prostacyclin metabolites }\end{array}$ & & & & & & $\mathrm{X}$ & & & & \\
\hline
\end{tabular}

Notes:

- Baseline visit is to be completed within 4 weeks prior to surgery. It is recommended that all baseline procedures occur at the same visit but consent and physical exam can occur on a separate day. Patients are to be randomised as close as possible to the time of the scheduled procedure and not more than 7 days before the planned procedure. Patients are to commence taking study medications on the day prior to the scheduled surgery. If the procedure is rescheduled, the patient is to cease taking medications if already started and to recommence the day before the rescheduled surgery.

\subsubsection{Clinical assessment of outcome}

Two separate observers will independently record the presence or absence of bruit at site of anastomosis. A standard stethoscope will be used (not a Doppler stethoscope). The bruit must be present over the site of anastomosis. In the event that the two observers disagree on the presence of the bruit, the bruit would be recorded as absent.

Bruit will be assessed by medical or nursing staff, specifically

- Consultant nephrologists 
- Advanced trainees

- Access surgeons

- Dialysis nurses

- Vascular access nurses

In addition, bruit assessment at discharge post surgery may be performed by recovery nurses.

\subsection{Adverse events}

Adverse events, including any cases of bleeding, will be managed as per usual local clinical care practice.

Adverse Events and Serious Adverse Events will be reported during study medication period and 3 months afterwards only (up until the Month 6 Visit). Only AEs that the site investigator rates as possibly or probably related with the study medication will be recorded. These will primarily include bleeding events and gastrointestinal symptoms. All SAEs will be recorded, whether related to the study medication or not. AEs and SAEs will be recorded in the regular data collection activities of the trial.

For the purposes of this trial the following definitions will apply;

Adverse Event:

An adverse event is defined as "any untoward medical occurrence in a patient or clinical investigation subject administered a pharmaceutical product which does not necessarily have a causal relationship with this treatment"

\section{Serious Adverse Event:}

A serious adverse event (experience) or reaction is any untoward medical occurrence that at any dose:

- results in death

- is life threatening (refers to an event in which the patient was at risk of death at the time of the event)

- requires inpatient hospitalisation or prolongation of existing hospitalisation

- results in persistent or significant disability/ incapacity

- is a congenital anomaly/ birth defect or

- is a medically important event or reaction.

Medical and scientific judgment should be exercised in deciding whether other situations should be considered serious such as important medical events that might not be immediately life-threatening or result in death or hospitalisation but might jeopardise the patient or might require intervention to prevent one of the other outcomes list in the definitions above.

Suspected Unexpected Serious Adverse Reaction (SUSAR)

Any adverse reaction that is both serious and suspected to be caused by the study medication that is not consistent with the information in the applicable product information (unexpected) is subject to expedited reporting to the relevant Regulatory Body.

Adverse events will be recorded in the regular data collection activities of the trial.

If the $A E$ is assessed as serious, the site PI must report the event to the Coordinating Office immediately or within 24 hours of being made aware of the event. The initial report should be made using the SAE Fax Notification sheet and an accompanying report that includes at a minimum: the name \& contact details of the reporter, the event \& 
seriousness criteria met, the patient's ID number \& treatment pack number and date the reporter became aware of the event.

If required, a more detailed follow-up report is to be submitted, within 7 days of the initial report submission, to the Coordinating Office and to the approving Human Research Ethics Committee (either local HREC or Lead HREC for state/country). Please refer to local site HREC guidelines as they may have a specific form they require for SAE reports.

The follow up written reports include: the patient's identification number, a description, seriousness and likely cause of the SAE, concomitant medications, duration of the SAE with start and end dates, and details of reporter.

A 24 hour contact number will operate to allow site staff to contact an appropriate representative of the Trial Management Committee if they believe breaking the blind is warranted. The representative will discuss and if necessary negotiate the necessity of breaking the blind. If this action is deemed necessary, the time, date and reason will be recorded.

\subsubsection{Reporting to Regulatory Authorities}

The responsibility for reporting serious, unexpected and associated adverse drug events to the relevant Regulatory Authority such as the Therapeutic Goods Administration in Australia lies with the study coordinating office (AKTN). The study coordinating office is also responsible for reporting all serious adverse events to Abbott Products and Bayer Drug Safety as per their contractual agreements.

\subsection{Substudies}

\subsubsection{RBC fatty acid substudy}

Omega-3 fatty acids incorporated into the red blood cell membrane will be used as a compliance measure. In addition, there will be a cross sectional assessment looking at $\mathrm{RBC}$ omega-3s and patient characteristics.

RBC fatty acids will be measured for all patients at participating sites before the commencement of study medications (at baseline), once the patient has been taking the study medications for 6 weeks and as close as possible to the last dose of study medications. 2-3 $\mathrm{ml}$ volume samples will be collected into EDTA, labelled with study number, patient initials and date of collection and stored frozen at -20 degrees or colder. Samples will be sent to the central testing laboratory in batches as advised. Complete details of collection, storage and shipping requirements are provided in the study operations manual.

This substudy will be undertaken by only the Australian and New Zealand sites.

\subsubsection{Dietary and physical questionnaire}

Many CKD patients have poor diets and malnutrition. This sub-study explores the status and the significance of dietary intake (including fish) and lifestyle factors in relation to patient outcomes. In particular, the questionnaire results will enable the interaction between these lifestyle and dietary factors and response to omega-3 fatty acids supplementation to be explored.

Trial participants will be asked to complete the dietary and physical questionnaire (appendix 13.4) once only, at baseline. Questionnaires will ideally be completed whilst the patient is in clinic and reviewed by the research nurse/clinical trial coordinator while 
the patient is still on site and any missing information completed at that time. Original responses should be kept in the patient's trial folder.

This substudy will be undertaken by all participating sites.

\subsubsection{Pre-surgical Doppler studies}

This sub-study explores whether pre-operative vascular characteristics determined by duplex ultrasound will predict vascular access outcomes either independently or in particular, the response to aspirin, omega-3 fatty acids and the combination.

Interested centres with vascular laboratories will perform standardised evaluation and reporting of arm vasculature by duplex ultrasound prior to surgery. The outline of the standardised methods for evaluation will be provided in the study operations manual.

This substudy will be undertaken by interested sites in any of participating countries with the requisite vein mapping faculties.

\subsubsection{Platelet and Coagulation Function}

This substudy aims to study the effects of aspirin and omega- 3 fatty acids alone and the combination on platelet function, coagulation and platelet activation as well as quantification of compliance with aspirin (prostaglandin metabolites).

At baseline and week 12 spot urine samples will be collected from participants with urinary output for the measurement of protein:creatinine ratio (to be tested by the local laboratory) and urinary thromboxane B2 and prostacyclin metabolites (a $5 \mathrm{~mL}$ sample stored at a temperature of $-20^{\circ} \mathrm{C}$ or lower, to be sent with the RBC fatty acid blood samples for testing by the central laboratory).

This substudy will be undertaken by only the Australian and New Zealand sites.

\section{Laboratory Procedures and Investigations}

\subsection{Sample handling and storage}

Apart from samples for the above substudies, all other routine blood and urine samples will be analysed by local laboratories, and samples will be collected in accordance with the requirements of these laboratories. The required sampling points for the routine blood tests are shown in table 5.3.1. For the blood samples taken at baseline and Week 12, patients must fast as per individual sites requirements for fasted lipid and glucose tests. Samples for the RBC fatty acid and platelet function substudies will be collected and stored as per section 5.6.1. Baseline blood samples must be tested within 4 weeks of AVF surgery. The routine blood tests required are:

1) Haematology and Coagulation: Full blood count (including $\mathrm{Hb}$, WCC and platelets) and coagulation (INR, APTT and fibrinogen).

2) Biochemistry: Creatinine, urea, electrolytes, calcium, phosphate, albumin, glucose, parathormone and $\mathrm{HbA} 1 \mathrm{c}$. The protein:creatinine ratio of patients with urine output will be tested.

3) Fasting Lipids: Total, LDL, HDL and triglycerides.

4) Optional tests (where these are routinely available) are fasting Homocysteine and Lipoprotein(a). 


\subsection{Imaging and other investigations}

Imaging and other investigations will be performed as per normal local practice.

\section{Data Management}

The timing of forms to be completed follows the schedule in Section 5.3.1 and is outlined in more detail in the operations manual.

Original consent forms are to be stored locally.

All source documents used in this trial, including medical records, should be stored for a period of 15 years following the close of the study.

\section{Quality Assurance}

\subsection{Training}

Assessment of bruit is to be performed according to guidelines provided.

\subsection{Measurement and laboratory accreditation}

A copy of the NATA or other accreditation certificate and local normal ranges will be sent to the coordinating centre prior to the use of any local laboratories.

\subsection{Site monitoring}

The coordinating centre reserves the right to visit sites for study monitoring purposes. At these visits, the authorised study monitor will require access to the medical records of study patients for the purpose of source data verification. The study monitor will also have access to all other study documentation, including ethics correspondence for the purpose of verifying appropriate study management.

\subsection{Auditing}

The coordinating centre reserves the right to audit sites. At these visits, the authorised study auditor will require access to the medical records of study patients.

\section{Statistical Considerations}

\subsection{Sample size}

\subsubsection{Primary Outcome: calculation}

The event rate for the primary outcome (AVF Access Failure) is estimated to be $30 \%$ at twelve months in the control group. To achieve a $30 \%$ relative risk reduction is achievable with omega- 3 fatty acids, then with $80 \%$ power and a significance level of $5 \%$, 954 study subjects will be required. This allows for $5 \%$ drop-in from placebo to active treatment, $5 \%$ drop-out from study treatment (to no treatment - assumed equivalent to

placebo), and $5 \%$ loss to follow-up. This is equivalent to an observed relative risk reduction of $24 \%$. Without adjustment for compliance, the study size needed would have been 734 subjects. 
The original $2 \times 2$ factorial design of the study would have resulted in equal number of patients distributed in both the parts of the study i.e. (1) aspirin hypothesis, and (2) omega-3 fatty acids hypothesis. The protocol amendment will allow inclusion of patients who are already taking and unable to cease aspirin into testing of the omega- 3 fatty acids hypothesis of the study. This will lead to disproportionate allocation of patients to two parts of the study.

We anticipate that at the end of study, 350 to 400 patients will be randomised to either aspirin or its placebo compared with 954 subjects allocated to omega-3 fatty acids or placebo. Thus, the resultant sample size will have adequate power to address the omega-3 fatty acids hypothesis but will not have adequate power to answer the aspirin hypothesis.

However, the assumption of event rate (AVF Access Failure) of $30 \%$ is very conservative. The actual event rate is expected to be between $30 \%$ and $50 \%$. With increasing event rate, required sample size will reduce as shown in table 4.

Table 4: The enrolment target with increasing event rates

\begin{tabular}{ccc}
\hline Event rate & \multicolumn{2}{c}{ Sample size } \\
Not & adjusted & adjusted \\
\hline $30 \%$ & 734 & 954 \\
\hline $40 \%$ & 488 & 634 \\
\hline $50 \%$ & 340 & 442 \\
\hline
\end{tabular}

The study is not adequately powered to detect a clinically important difference between the combination of aspirin and omega-3 fatty acids and either treatment alone, but preliminary data on the combination especially on adverse events will be obtained.

\subsubsection{Primary Outcome: justification of assumptions used}

The event rate of the primary outcome, AVF Access Failure, is based on review of all papers published in this area. The effect size, $30 \%$ reduction, is based on the smallest size of the effect the proposing clinicians believe would lead to a change in practice (incorporation of intervention) should the trial prove the intervention is efficacious. AVF Access Failure is preferred as a primary outcome measure over failure of patency because it is more clinically relevant.

\subsubsection{Planned recruitment rate}

During the initial planning for the study, a review of the data from the ANZDATA renal registry [41] showed that 7,202 patients were receiving dialysis in Australia and New Zealand in December 2004 with 2042 patients commencing dialysis in Australia and New Zealand in that year. Data obtained from the New Zealand renal population and Princess Alexandra Hospital, Queensland, revealed that approximately 1 procedure was performed for every 2.3 - 2.8 patients receiving haemodialysis (total numbers of patients - new patients and prevalent patients). The New Zealand data was obtained by Dr. Mark Marshall (survey between October 2000 and September 2001) and by Dr. Carmel Hawley (Queensland) based on prospectively collected data in PAH renal unit from January 2004 until December 2004. In addition information from the 2005 registry report supports this data.

It was expected that all incident patients require new vascular access, in addition to existing patients on dialysis who needed further access because of failure of previous 
vascular access procedures. Twenty seven percent of haemodialysis patients in Australia and $33 \%$ of patients in New Zealand, respectively, were incident patients in 2004. With extrapolation to the whole Australian and New Zealand renal population, and estimating 1 procedure per 3 patients allowed us to derive that a total of 2,400 access procedures are performed each year (AVF and AVG). With approximately $75 \%$ of access procedures AVF, it was estimated that there was 1,800 de-novo AVF creations per year. Assuming we were able to recruit $22 \%$ of these, recruitment for the study was estimated to take approximately three years.

After almost two years of recruitment for the study, patient enrolment has been much lower than expected, with 154 patients enrolled which is $13 \%$ of the total recruitment target. The first avenue to increase recruitment was the inclusion of additional sites outside Australia and New Zealand. Sites from Malaysia and the United Kingdom have been included as they have a good track record for recruitment into other renal studies, had a similar renal cohort and treatment regime and would add significant numbers of patients to the study.

Additionally, an analysis of screening logs showed that there was a high rate of screening failure with $73 \%$ of patients screened in Australia and New Zealand being excluded. The commonest cause of exclusion was current use of aspirin in $37 \%$ of patients excluded. Estimates from Malaysian and United Kingdom sites indicate that aspirin usage is also high in their renal populations $(30-40 \%)$. With a aim of removing this barrier to recruitment, two additional arms have been added to the study which will allow patients who are taking aspirin and are unable to cease (open label aspirin) to be randomised to omega-3 fatty acids or placebo only. It is estimated that this change will improve the recruitment rate by at least double. This improvement combined with the addition of the new overseas sites should mean that the recruitment period will only be extended by 18 months from the original recruitment period of 3 years.

\subsection{Analysis}

\subsubsection{Definition of Populations for Analyses}

Analysis of the efficacy outcomes will be on an intent-to-treat basis. All randomised patients will be analysed in the group they were allocated to, even if they do not receive treatment as allocated, or do not commence treatment. All randomised patients will be included in the analysis.

A marginal analysis will be conducted for efficacy outcomes. Tests of hypotheses will be at the $5 \%$ significance level, and 2-sided p-values will be used. A statistical analysis plan (SAP) will be written by a blinded statistician and reviewed by the principal investigators (also blinded). It will be this document that the statistical analysis methods outlined in the next paragraphs will be fully developed.

\subsubsection{Primary analysis methods}

The efficacy analysis will involve several marginal comparisons:

a) Omega-3 fatty acids vs. placebo (with and without aspirin). This will be a comparison of Arms $(A+B+E)$ vs. $(C+D+F)$. Please refer to section 1.1 for further details

b) Aspirin vs. placebo excluding patients who are unable to cease aspirin. This will be a comparison of Arms $(A+C)$ vs. $(B+D)$.

c) Omega-3 fatty acids vs. placebo omega-3 fatty acids in open-label aspirin patients (Arms E vs. F). 
The primary outcome is measured as the proportion with AVF Access Failure. The primary hypothesis will be tested using a chi-square test of proportions in a marginal analysis. (comparison A above). A similar strategy will be performed for all binary secondary efficacy objectives. For survival endpoints the log-rank test will be used in the same marginal analysis.

The exploratory efficacy objective is more investigative and involves a marginal comparison of type B for the primary and all secondary efficacy endpoints. A same strategy as before will be used.

An adjusted analysis based on logistic regression or the Cox model will be performed, including the stratification variables in the model for respectively binary and survival endpoints.

\subsubsection{Safety analyses}

Serious Adverse Events will be tabulated by treatment arm (n, \%) and various marginal totals of patients experiencing a particular category of SAE will be presented. The same marginal analyses (type A, B or C) will be considered. Where applicable, Chi-square or Fisher tests will be used to test potential differences in the different marginal comparisons.

\subsubsection{Subgroup analyses}

Subgroup analysis (omega-3 fatty acids vs. placebo omega- 3 fatty acids) stratified by aspirin use (patients unable to cease aspirin versus the others) will also be conducted using the same techniques as above.

\subsubsection{Interim analyses and trial stopping rules}

Safety reports will be produced regularly and sent to the Data and Safety Monitoring Board (DSMB). Two formal interim analyses will be conducted after $1 / 3$ and $2 / 3$ of total patients have been accrued and followed for sufficient time. The Haybittle-Peto rule will be used as a stopping guideline for efficacy as it offers the flexibility to update the number of looks if need be. If two interim analyses equally-spaced are indeed performed, the level of the final analysis will be 0.048 . Similar analyses to the primary one will be undertaken on the secondary outcomes, as well as exploratory analyses looking at the validity of the chosen primary outcomes and its correlation with other outcomes, and the prediction of thrombosis from baseline variables such as markers of inflammation. SAEs or AEs will also be formally examined by the DSMB. Arrangements will be made to provide the Chair of the DSMB and possibly other members with regular information on fatalities and life-threatening SAEs possibly related to trial treatment (e.g. intracranial haemorrhage).

\section{Ethical Considerations}

\subsection{Adherence to regulations and guidelines}

This trial will adhere to the regulations, guidelines and standards espoused in the World Medical Association Declaration of Helsinki, the NHMRC National Statement of Ethical Conduct in Research Involving Humans, the joint NHMRC/AVCC Statement and Guidelines on Research Practice, and the Therapeutic Goods Administration Note for Guidance on Good Clinical Practice. 


\subsection{Ethics committee approvals}

Patients will not be enrolled until the final approved protocol, as well as any trial information for patients is reviewed and approved by a Human Research Ethics Committee.

Correspondence with HRECs should be copied and supplied to the coordinating centre. It is the responsibility of the investigators to ensure that the appropriate person from each site is aware of the reporting responsibilities of each site to the relevant HRECs.

\subsection{Justification of potential risks and benefits}

The potential benefits to the patients are those relating to a reduction in AVF thrombosis rate and the associated benefits of lower hospitalisation rate, lower risk of needing alternative vascular access procedures and further attempts at establishing permanent vascular access. The only significant concern in this study is that of bleeding complications. This risk relates primarily to the group assigned to aspirin alone and to the group assigned combined aspirin and omega-3 fatty acid therapy.

The relationship between aspirin use and an increased risk of bleeding is a potential concern in patients with CKD. In the general population, the use of a chronic low dose of aspirin doubles the risk of serious gastrointestinal bleeding [33] and the theoretical risk may be higher in patients with CKD because of the presence of uraemic induced impairment of haemostasis. There have been published studies [34, 35] showing a significant elevation in bleeding times in patients on haemodialysis treatments administered a single dose of aspirin, but surprisingly little evidence-based clinical trial data in this population. This has been explored in the UK-HARP-I study. In this 2x2 factorial design study involving simvastatin and aspirin as active therapies, allocation to the aspirin group was not associated with an excess of major bleeds $(2 \%$ in patients on aspirin vs. $3 \%$ in patients not on aspirin), but there was a three-fold increase in minor bleeding [36]. Importantly though, in this study, patients received aspirin for 12 months, whereas our study has a much shorter period on therapy of 3 months. In addition, patients with ESRD on dialysis undergoing renal transplantation have successfully received pre-operative aspirin for the prevention of graft thrombosis without an increased risk of major bleeding $[42,43]$.

Omega-3 fatty acids appear to be well tolerated, even in high doses, with gastrointestinal complaints particularly nausea, vomiting, diarrhoea and non-specific discomfort being the most often reported. In studies looking at the effect of omega-3 fatty acids on bleeding time it has not been found to be significantly prolonged [37]. In studies that have been done thus far in the CKD cohort, there have been no clear effects demonstrated in relation to platelet aggregation and bleeding times. There has only been one report of a serious bleeding event in a single patient in an uncontrolled study [38]. Furthermore, it has been reported that administration of omega-3 fatty acids can protect the gastric mucosa against aspirin-induced injury, the postulated mechanism being that omega- 3 fatty acids counteract the effect of aspirin on the decrease in prostacyclin (combined effect of $\mathrm{PGI}_{2}$ and $\mathrm{PGI}_{3}$ ) [40]. This is supported by clinical data suggesting that omega-3 fatty acids decrease gastric erosions and ulcers caused by aspirin or alcohol [39]. Thus the combination of aspirin and omega- 3 fatty acids may be expected to be additive in terms of efficacy in vascular access thrombosis, but to have a lower risk of bleeding complications than the use of aspirin alone. 
It is important to perform this study to carefully explore the risks and benefits of aspirin and omega-3 fatty acids as single agents or as combination therapy.

\subsection{Protection of patient confidentiality}

Patients' records and the data generated by the study will be confidential in line with the recommendations of the NHMRC and the 2001 privacy legislation. Any information that may identify a patient will be excluded from data presented in the public arena. Data must be stored in a secure, lockable location. Electronic data storage must have adequate password protection. Standardised case report forms will be provided for each subject on this study. The participants in this study will be identified only by initials and subject number on these forms. De-identified information may only be released to the AKTN or designee.

\subsection{Insurance}

Insurance will be provided through the University of Queensland.

\section{Trial Management}

\subsection{Organisational structure}

The Trial Management Committee (TMC) is comprised of persons appointed through the AKTN Standard Operating Procedure. Representatives include the trial proposer (chair), one member of the AKTN Scientific Committee, study biostatistician, a member of the AKTN Operations Secretariat, and others as required. It is the responsibility of this group to provide leadership to the overall conduct of the trial. In particular, this group will review the progress of the study in achieving the objectives, take appropriate decisions to meet these objectives, and make decisions regarding the continuation or modification of a trial given reports from the Safety and Data monitoring committee.

Specifically, the TMC will:

- Monitor the blinded event rate for comparison with the one used for design purposes

- Monitor missing data

- Forward to the DSMB, on an ongoing basis, details of treatment-emergent deaths and life-threatening SAEs possibly related to treatment

- Ensure that treatment withdrawals are continuously monitored

- Communicate any notable imbalance between treatment groups to the DSMB, including details on the reason for withdrawal

The TMC oversees the trial-related activities of the coordinating office. The TMC reports to the AKTN Scientific Committee.

The Data and Safety Monitoring Board (DSMB) will be comprise of a statistician, an experienced clinical trialist and two Nephrologists who have no involvement with the day to day running of the study. The DSMB will be responsible for reviewing the interim analyses of safety (to be conducted at $1 / 3$ and $2 / 3$ of total patient numbers accrual), and will advise the Trial Management Committee when the stopping rules have reached or any other matters of safety that arise during the course of the study. 


\subsection{Publication Policy}

The Australasian Kidney Trial Network subscribes to the criteria for authorship formulated by the International Committee of Medical Journal Editors [44]:

"Each author should have participated sufficiently in the work to take public responsibility for the content. Authorship credit should be based only on substantial contributions to

(a) conception and design, or analysis and interpretation of data; and to

(b) drafting the article or revising it critically for important intellectual content; and on

(c) final approval of the version to be published.

Conditions (a), (b), and (c) must all be met."

The main authorship will appear as follows:

The writing committee (listed: Chief PI listed first, then remaining members listed alphabetically) for the FAVOURED Study Collaborative Group.

On the front page, the following will be listed:

Members of the FAVOURED Study Collaborative Group are listed in the Supplementary Appendix, available with the full text of this article.

Authorship in the appendix will appear in the following order:

Writing Committee

Trial Management Committee

Steering Committee (the PI at each site)

Project Management Team (AKTN)

Data and Safety Monitoring Board

Data Management / Information Technology

All investigators and Study Coordinators at each site

The TMC will approve all publications arising from the trial before submission to journals, as will the AKTN Scientific Committee or its delegate.

Please see the AKTN Publication policy for more detail on authorship. 


\section{References}

1. Allon, M. and M.L. Robbin, Increasing arteriovenous fistulas in hemodialysis patients: Problems and solutions. Kidney International, 2002. 62(4): p. 1109-1124.

2. Polkinghorne, K.R., et al., Vascular access and all-cause mortality: a propensity score analysis. Journal of the American Society of Nephrology, 2004. 15(2): p. 477-86.

3. Pastan, S., J.M. Soucie, and W.M. McClellan, Vascular access and increased risk of death among hemodialysis patients. Kidney International, 2002. 62(2): p. 620-626.

4. Excell, L., M. Marshall, and S.P. McDonald, Haemodialysis, p35-52. In: ANZDATA Registry Report 2004, L. Excell and S.P. McDonald, Editors. 2004, Australia and New Zealand Dialysis and Transplant Registry: Adelaide, South Australia.

5. Manns, B., et al., Establishment and maintenance of vascular access in incident hemodialysis patients: a prospective cost analysis. Journal of the American Society of Nephrology, 2005. 16(1): p. 201-9.

6. Port, F.K., et al., Improving outcomes for dialysis patients in the international Dialysis Outcomes and Practice Patterns Study. Clinical Journal of the American Society of Nephrology, 2006. 1(2): p. 246-55.

7. Ishani, A., et al., Septicemia, access and cardiovascular disease in dialysis patients: the USRDS Wave 2 study. Kidney International, 2005. 68(1): p. 311-8.

8. Asif, A., P. Roy-Chaudhury, and G.A. Beathard, Early arteriovenous fistula failure: a logical proposal for when and how to intervene. Clinical Journal of the American Society of Nephrology, 2006. 1(2): p. 332-9.

9. Rooijens, P.P.G.M., et al., Radiocephalic Wrist Arteriovenous Fistula for Hemodialysis: Meta-analysis Indicates a High Primary Failure Rate. European Journal of Vascular and Endovascular Surgery, 2004. 28(6): p. 583-589.

10. Marshall, M., et al., Haemodialysis, in ANZDATA Registry Report 2005. 2005, Australia and New Zealand Dialysis and Transplant Registry: Adelaide. p. 61-81.

11. Sidawy, A.N., et al., Recommended standards for reports dealing with arteriovenous hemodialysis accesses. Journal of Vascular Surgery, 2002. 35(3): p. 603-610.

12. Gröntoft, K.-C., et al., Effects of Ticlopidine in AV-fistula Surgery in Uremia. Scandinavian Journal of Urology and Nephrology, 1998. 32(4): p. 276-283.

13. Kaufman, J.S., Antithrombotic agents and the prevention of access thrombosis. Seminars in dialysis, 2000. 13(1): p. 40-46.

14. Andrassy, K., et al., Prevention of p.o. clotting of av. cimino fistulae with acetylsalicyl acid. Results of a prospective double blind study. Klin Wochenschr, 1974. 52: p. 348349.

15. Kooistra, M.P., et al., Low-dose aspirin does not prevent thrombovascular accidents in low-risk haemodialysis patients during treatment with recombinant human erythropoietin. Nephrology, Dialysis, Transplantation, 1994. 9(8): p. 1115-1120.

16. Schmitz, P.G., et al., Prophylaxis of hemodialysis graft thrombosis with fish oil: Doubleblind, randomized, prospective trial. Journal of the American Society of Nephrology, 2002. 13(1): p. 184-190.

AKTN Protocol - FAVOURED Study

Version 9 30/11/2010 
17. Engstrom, K., R. Wallin, and T. Saldeen, Effect of low-dose aspirin in combination with stable fish oil on whole blood production of eicosanoids. Prostaglandins Leukotrienes and Essential Fatty Acids, 2001. 64(6): p. 291-297.

18. Khajehdehi, P., Lipid-lowering effect of polyunsaturated fatty acids in hemodialysis patients. Journal of renal nutrition, 2000. 10(4): p. 191-195.

19. Ando, M., T. Sanaka, and H. Nihei, Eicosapentanoic acid reduces plasma levels of remnant lipoproteins and prevents in vivo peroxidation of $L D L$ in dialysis patients. Journal of the American Society of Nephrology, 1999. 10(10): p. 2177-2184.

20. Beilin, L.J. and T.A. Mori, Dietary omega-3 fatty acids, in Lifestyle Modification for the Prevention and Treatment of Hypertension, P. Whelton, J. He, and G. Louis, Editors. 2003, Marcel Dekker Inc: New York. p. 273-300.

21. Mori, T.A. and L.J. Beilin, Omega-3 fatty acids and inflammation. Current Atherosclerosis Reports, 2004. 6(6): p. 461-7.

22. Mori, T.A., Effect of fish and fish oil-derived omega-3 fatty acids on lipid oxidation. Redox Report, 2004. 9(4): p. 193-7.

23. Friedman, A. and S. Moe, Review of the effects of omega-3 supplementation in dialysis patients. Clinical Journal of the American Society of Nephrology, 2006. 1(2): p. 182-92.

24. Ethier, J., et al., Aspirin prescription and outcomes in hemodialysis patients: the Dialysis Outcomes and Practice Patterns Study (DOPPS). American journal of kidney diseases : the official journal of the National Kidney Foundation, 2007. 50(4): p. 602-11.

25. Hasegawa, T., et al., Consistent aspirin use associated with improved arteriovenous fistula survival among incident hemodialysis patients in the dialysis outcomes and practice patterns study. Clinical journal of the American Society of Nephrology : CJASN, 2008. 3(5): p. 1373-8.

26. Chan, K.E., et al., Anticoagulant and antiplatelet usage associates with mortality among hemodialysis patients. Journal of the American Society of Nephrology : JASN, 2009. 20(4): p. 872-81.

27. Dember, L.M., et al., Effect of clopidogrel on early failure of arteriovenous fistulas for hemodialysis: a randomized controlled trial. JAMA, 2008. 299(18): p. 2164-71.

28. Woodman, R.J., et al., Effects of purified eicosapentaenoic acid and docosahexaenoic acid on platelet, fibrinolytic and vascular function in hypertensive type 2 diabetic patients. Atherosclerosis, 2003. 166(1): p. 85-93.

29. Woodman, R.J., et al., Effects of purified eicosapentaenoic and docosahexaenoic acids on glycemic control, blood pressure, and serum lipids in type 2 diabetic patients with treated hypertension. American Journal of Clinical Nutrition, 2002. 76(5): p. 1007-1015.

30. Chan, D.C., et al., Factorial study of the effects of atorvastatin and fish oil on dyslipidaemia in visceral obesity. European Journal of Clinical Investigation, 2002. 32(6): p. 429-436.

31. Mori, T.A., et al., Differential effects of eicosapentaenoic acid and docosahexaenoic acid on vascular reactivity of the forearm microcirculation in hyperlipidemic, overweight men. Circulation, 2000. 102(11): p. 1264-1269.

AKTN Protocol - FAVOURED Study

Version 9 30/11/2010

Page 31 of 50 
32. Mori, T.A., et al., Purified eicosapentaenoic and docosahexaenoic acids have differential effects on serum lipids and lipoproteins, LDL particle size, glucose, and insulin in mildly hypedipidemic men. American Journal of Clinical Nutrition, 2000. 71(5): p. 1085-1094.

33. de Abajo, F.J. and L.A. Garcia Rodriguez, Risk of upper gastrointestinal bleeding and perforation associated with low-dose aspirin as plain and enteric-coated formulations. BMC clinical pharmacology, 2001. 1: p. 1-1.

34. Gaspari, F., et al., Aspirin prolongs bleeding time in uremia by a mechanism distinct from platelet cyclooxygenase inhibition. J. Clin. Invest, 1987. 79: p. 1788-1797.

35. Livio, M., G. Vigano, and A. Benigni, Moderate doses of aspirin and risk of bleeding in renal failure. Lancet, 1986. 1(8478): p. 414-416.

36. Baigent, C., et al., First United Kingdom Heart and Renal Protection (UK-HARP-I) study: biochemical efficacy and safety of simvastatin and safety of low-dose aspirin in chronic kidney disease. American Journal of Kidney Diseases, 2005. 45(3): p. 473-84.

37. Svaneborg, N., et al., The acute and short-time effect of supplementation with the combination of $n-3$ fatty acids and acetylsalicylic acid on platelet function and plasma lipids. Thrombosis Research, 2002. 105(4): p. 311-316.

38. Diskin, C.J., et al., Fish oil to prevent intimal hyperplasia and access thrombosis. Nephron, 1990. 55(4): p. 445-447.

39. Szabo, S. and C. Rogers, Diet, ulcer disease, and fish oil. Lancet, 1988. 331(8577): p. 119.

40. Al-Harbi, M.M., et al., Effect of acute administration of fish oil (omega-3 marine triglyceride) on gastric ulceration and secretion induced by various ulcerogenic and necrotizing agents in rats. Food and Chemical Toxicology, 1995. 33(7): p. 553-558.

41. McDonald, S.P., L. Excell, and V. Shtangey, Method and Location of Dialysis, in ANZDATA Registry Report 2005. 2005, Australia and New Zealand Dialysis and Transplant Registry: Adelaide. p. 54-60.

42. Robertson, A.J., et al., Low dose aspirin as prophylaxis against renal-vein thrombosis in renal-transplant recipients. Nephrol. Dial. Transplant., 2000. 15(11): p. 1865-1868.

43. Murphy, G.J., et al., Influence of aspirin on early allograft thrombosis and chronic allograft nephropathy following renal transplantation. British Journal of Surgery, 2001. 88(2): p. 261-266.

44. Guidelines on authorship. International Committee of Medical Journal Editors. British medical journal (Clinical research ed ), 1985. 291(6497): p. 722. 


\section{Appendices}

13.1 Sample Patient Information Sheet (Patients randomised to omega-3 fatty acids and aspirin)

(On institution letterhead)

(Name of Institution)

PATIENT INFORMATION SHEET

Patients taking Omega-3 Fatty Acid \& Aspirin or Placebo

\section{A randomised, double-blind, placebo-controlled, factorial-design trial to assess the effect of aspirin and fish oil (omega- 3 fatty acids) in the prevention of early thrombosis in arterio-venous fistulae in patients with Stage IV or V chronic kidney disease requiring haemodialysis}

\section{FAVOURED Study (Fish oil and Aspirin in Vascular access OUtcomes In REnal Disease}

\section{Purpose of this form}

This form is meant to help you to understand the information about the study. Your doctor should have already discussed the study with you. This form is intended to help you to decide whether or not to take part in the study by telling you about what is involved and what the possible risks and benefits of this study.

\section{Purpose of the study:}

The aim of the study is to find out whether aspirin or fish oil helps reduce blood clots in arteriovenous fistulae. Arterio-venous fistula or "AVF" is where an artery and vein are joined in your arm so that you are able to start haemodialysis.

\section{Why is the study being done?}

One problem which can occur with your AVF is if a blood clot forms and blocks blood flow. If this happens, it will usually happen within a few weeks of the surgery. One way to try and prevent this happening is to use medicines to prevent clots forming.

Platelets are cells in your blood which are involved in forming clots. Aspirin is well-known to have anti-platelet effects. Fish oil also has a number of effects may help stop clots forming. A combination of aspirin and fish oil may help stop clots forming better than either medication by itself.

Who will be asked to enter the study?

You will be invited to participate in the trial if you will shortly having an AVF created in your arm and are over 19 years old. 


\section{What will happen on this study?}

\section{Visit 1 - Baseline Visit (up to 4 weeks prior to surgery)}

At this visit, you will be asked to provide written informed consent to participate in the study. Information will be collected such as:

- Your height

- Your weight

- Your blood pressure

- Any relevant medical history (including past use of dialysis and other medical conditions)

- Any medications you currently take

A fasting blood sample will be taken. You will be asked to complete a questionnaire about your lifestyle and dietary habits.

\section{Visit 2 - Randomisation Visit (up to 7 days prior to surgery)}

At this visit, you will be given the study medication. You will be asked to take study medication every day from the day before your AVF procedure for 3 months.

The daily medication will be

- One tablet of aspirin (100 mg) or its placebo, and

- Four capsules of fish oil (4 grams) or its placebo.

The placebo or dummy medication look just like the actual medication but contain no active ingredients. These amounts have proven to be effective in other studies.

You will be asked to take 2 capsules of fish oil and one tablet aspirin just before your breakfast and 2 capsules of fish oil just before your evening meal daily. Which type of medication you take will be decided at random (like a throw of the dice). You will have a 1 in 4 chance of receiving aspirin and fish oil together, aspirin alone, fish oil alone or just placebo. None of the study staff or any member of your usual health care team will know which medication you are taking.

While you are taking the study medication, please do not take any other medication which contains aspirin or fish oil. Medications which contain aspirin can include:

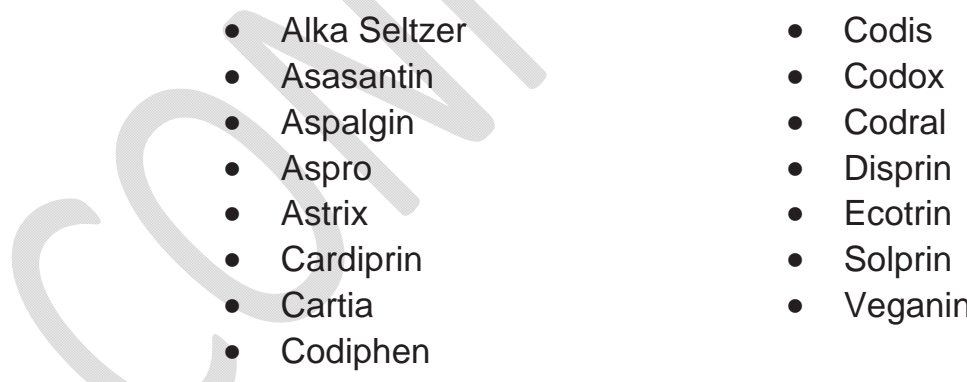

If you have a question about a medication you think may contain aspirin or fish oil, please contact your trial coordinator (insert name and phone number.)

Some herbal medications should not be taken with aspirin. So please be cautious when taking these medications while taking the study medication. It is also recommended that you limit the amount of alcohol you drink while you are taking the study medication.

Visit 3 - AVF Surgery (1 day after starting medication)

On the day of your AVF procedure, a description of the procedure will be collected including the results of any vein mapping studies undertaken prior to surgery. Details such as height, weight, blood pressure, any changes to concomitant medicine or health will be reported. 
Visits 4, 5 \& 6 - Treatment Visits (Weeks 1, 6 \& 12)

At these visits, details about you will be recorded. This information includes:

- Your weight

- Your blood pressure

- Your use of the study medication

- Whether you are dialysing or not

- The status of your AVF

- Any changes to your health or medications you are taking

At visit 5 and 6 (weeks 6 and 12) a blood sample will be collected (you will need to fast for the week 12 sample).

Visit 7 onwards - Follow-up Visits (Month 6, Years 1, 2 and 3)

In this part of the study, you will have visits at 6 month, 1 year, 2 year and 3 years. At these visits, details such as weight, blood pressure, your dialysis and AVF status and at month 6 any changes to your health or concomitant medications will be recorded.

Throughout the study, staff will regularly check your AVF for "bruit", the sound the blood normally makes if the AVF is working properly. No other aspect of your medical care will be altered in any way. You will still be looked after by your usual health care team. A summary of all data that will be recorded is in the table below.

\begin{tabular}{|c|c|c|c|c|c|c|c|c|c|c|}
\hline Study Phase & Baseline & Randomisation & Surgery & & atm & $\begin{array}{l}\text { nt } \\
\text { ks) }\end{array}$ & & ow & $p(m$ & hs) \\
\hline Assessment & & & & 1 & 6 & 12 & 6 & 12 & 24 & 36 \\
\hline Study medication dispensed & & $\mathbf{x}$ & & & & & & & & \\
\hline Informed consent & $\mathrm{X}$ & & 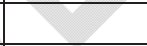 & & & & & & & \\
\hline Medical history & $\mathrm{X}$ & & & & & & & & & \\
\hline Weight & $\mathrm{x}$ & & $x$ & $\mathbf{x}$ & $\mathrm{X}$ & $\mathbf{x}$ & $x$ & $\mathrm{X}$ & $\mathbf{x}$ & $\mathbf{x}$ \\
\hline Blood pressure & $x$ & 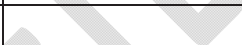 & $x$ & $\mathbf{x}$ & $\mathbf{x}$ & $\mathbf{x}$ & $x$ & $\mathbf{x}$ & $\mathbf{x}$ & $x$ \\
\hline Demographics & $x$ & 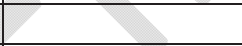 & & & & & & & & \\
\hline Physical exam & $\mathrm{X}$ & & & & & & & & & \\
\hline Dialysis details & $\mathrm{x}$ & 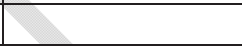 & & $\mathbf{x}$ & $\mathrm{X}$ & $\mathbf{x}$ & $\mathrm{X}$ & $\mathrm{X}$ & $x$ & $\mathbf{x}$ \\
\hline $\begin{array}{l}\text { Description and status of } \\
\text { vascular access }\end{array}$ & $x$ & 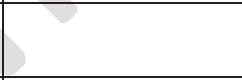 & $x$ & $x$ & $x$ & $x$ & $\mathbf{X}$ & $\mathbf{x}$ & $\mathbf{x}$ & $\mathbf{x}$ \\
\hline Concomitant meds & $\mathbf{x}$ & & $\mathbf{x}$ & $\mathbf{x}$ & $\mathbf{X}$ & $\mathbf{x}$ & $x$ & & & \\
\hline Adverse events & $x$ & & $x$ & $\mathbf{x}$ & $\mathbf{x}$ & $x$ & $x$ & & & \\
\hline Fasting blood test & $x$ & & & & $x$ & $x$ & & & & \\
\hline
\end{tabular}

\section{Are there any risks?}

There is a large amount of research about the use of aspirin for prevention of blood clots. In general, aspirin is very good in stopping blood clots and there is only a small possibility of bleeding while taking aspirin. This study uses low dose aspirin (100mg once a day). The risk of side effects is very small at this dose. However, your doctor will keep a close eye on you and you should report any unusual bleeding immediately to your renal unit nurses or nephrologist. Symptoms that could have serious consequences include black stools, feeling faint, signs of allergic reactions and abdominal pain.

Fish oil is generally doesn't have many side effects. Gastrointestinal side effects such as nausea, vomiting, diarrhoea and non-specific discomfort are the most commonly reported. 
These problems can be reduced by taking the fish oil capsules with a glass of cold water. The combination of aspirin and fish oil should have a lower risk of bleeding than just taking aspirin.

If you are a woman who is able to become pregnant, you should use an effective method of birth control for the three months that you are taking the study medication. If you do become pregnant, you should stop all study medications and inform your renal unit nurses or nephrologist immediately. The study medications are not known to affect men fathering children.

\section{Potential Benefits}

If you decide to take part in this trial, your medical condition will be very closely monitored. Any changes in your health will be very quickly identified. The results from this study may help other haemodialysis patients having AVFs in the future.

\section{Confidentiality}

All information collected for this study will be treated with complete confidentiality. You will be asked to give permission to any regulatory authorities related to the study to have access your medical records. This information will only be used for purposes of this study. Information gathered by the ANZDATA Registry (the Australia and New Zealand Dialysis and Transplant Registry) may also be used as part of this study. Medical records will be stored for accessibility for at least 15 years.

\section{Costs}

Your involvement in this study will not cost you anything. You will not be paid to take part in this study. Taking part in this study will not change usual hospital liability arrangements and processes.

\section{Do you have a choice?}

Entry into this study is entirely voluntary. Choosing not to take part in this study will not affect your treatment or care in any way. The doctors will continue to treat you with the best means available.

If you agree to participate in this study you will be asked to sign a consent form. However you may withdraw from the study at any time without giving a reason. Your doctor may also decide that you should stop if they believe it is in your best interest. You will be told if any new information arises which might affect your decision to be in the study.

For further information about this study, please contact (medical contact, research nurse contact, phone numbers).

\section{Ethics Approval}

This study has been reviewed and approved by the (local) Ethics Committee. Should you wish to discuss the matter with someone not directly involved, you can contact (Ethics Committee contact, number).

This study has been cleared by one of the human ethics committees of the University of Queensland in accordance with the National Health and Medical Research Council's guidelines. You are of course, free to discuss your participation in this study with project staff (contactable on ......................). If you would like to speak to an officer of the University not involved in the study, you may contact the Ethics Officer on 0733653924. 
13.2 Sample Patient Information Sheet (Patients randomised to omega-3 fatty acids only)

(On institution letterhead)

(Name of Institution)

PATIENT INFORMATION SHEET

Patients taking Omega-3 Fatty Acids/Placebo and Open Label Aspirin

\begin{abstract}
A randomised, double-blind, placebo-controlled, factorial-design trial to assess the effect of aspirin and fish oil (omega-3 fatty acids) in the prevention of early thrombosis in arterio-venous fistulae in patients with Stage IV or V chronic kidney disease requiring haemodialysis
\end{abstract}

FAVOURED Study (Fish oil and Aspirin in Vascular access OUtcomes In REnal Disease

\title{
Purpose of this form
}

This form is meant to help you to understand the information about the study. Your doctor should have already discussed the study with you. This form is intended to help you to decide whether or not to take part in the study by telling you about what is involved and what the possible risks and benefits of this study.

\section{Purpose of the study:}

The aim of the study is to find out whether aspirin and fish oil helps reduce blood clots in arteriovenous fistulae. Arterio-venous fistula or "AVF" is where an artery and vein are joined in your arm so that you are able to start haemodialysis.

\section{Why is the study being done?}

One problem which can occur with your AVF is if a blood clot forms and blocks blood flow. If this happens, it will usually happen within a few weeks of the surgery. One way to try and prevent this happening is to use medicines that may prevent the clot forming.

Platelets are cells in your blood which are involved in forming clots. Aspirin is well-known to have anti-platelet effects. Fish oil also has a number of effects may help stop clots forming. A combination of aspirin and fish oil may help stop clots forming better than either medication by itself.

Who will be asked to enter the study?

You will be invited to participate in the trial if you will shortly having an AVF created in your arm and are over 19 years old. 


\section{What will happen on this study?}

\section{Visit 1 - Baseline Visit (up to 4 weeks prior to surgery)}

At this visit, you will be asked to provide written informed consent to participate in the study. Information will be collected such as:

- Your height

- Your weight

- Your blood pressure

- Any relevant medical history (including past use of dialysis and other medical conditions)

- Any medications you currently take

A fasting blood sample will be taken. You will be asked to complete a questionnaire about your lifestyle and dietary habits.

\section{Visit 2 - Randomisation Visit (up to 7 days prior to surgery)}

At this visit, you will be given the study medication. You will be asked to take study medication every day from the day before your AVF procedure for 3 months. The daily medication will be four capsules of fish oil (4 grams) or its placebo. The placebo or dummy medication look just like the fish oil capsules but contain no active ingredients. This amount has been proven to be effective in other studies.

You will be asked to take 2 capsules of fish oil just before your breakfast and 2 capsules of fish oil just before your evening meal daily. Which type of medication you take will be decided at random (like a throw of the dice). You will have a 1 in 2 chance of receiving fish oil alone or placebo. None of the study staff or any member of your usual health care team will know which medication you are taking.

While you are taking the study medication, please do not take any other medication which contains fish oil. Please inform the study staff if you take any medications containing aspirin other than your regular low dose aspirin including:

- Alka Seltzer

- Asasantin

- Aspalgin

- Aspro

- Astrix

- Cardiprin

- Cartia

- Codiphen
- Codis

- Codox

- Codral

- Disprin

- Ecotrin

- Solprin

- Veganin.

If you have a question about a medication you think may contain aspirin or fish oil, please contact your trial coordinator (insert name and phone number.)

Some herbal medications should not be taken with aspirin. So please be cautious when taking these medications while taking the study medication. It is also recommended that you limit the amount of alcohol you drink while you are taking the study medication.

Visit 3 - AVF Surgery (1 day after starting medication)

On the day of your AVF procedure, a description of the procedure will be collected including the results of any vein mapping studies undertaken prior to surgery. Details such as height, weight, blood pressure, any changes to concomitant medicine or health will be reported. 
Visits 4, 5 \& 6 - Treatment Visits (Weeks 1, 6 \& 12)

At these visits, details about you will be recorded. This information includes:

- Your weight

- Your blood pressure

- Your use of the study medication

- Whether you are dialysing or not

- The status of your AVF

- Any changes to your health or medications you are taking

At visit 5 and 6 (weeks 6 and 12) a blood sample will be collected (you will need to fast for the week 12 sample).

Visit 7 onwards - Follow-up Visits (Month 6, Years 1, 2 and 3)

In this part of the study, you will have visits at 6 month, 1 year, 2 year and 3 years. At these visits, details such as weight, blood pressure, your dialysis and AVF status and at month 6 any changes to your health or concomitant medications will be recorded.

Throughout the study, staff will regularly check your AVF for "bruit", the sound the blood normally makes if the AVF is working properly. No other aspect of your medical care will be altered in any way. You will still be looked after by your usual health care team. A summary of all data that will be recorded is in the table below.

\begin{tabular}{|c|c|c|c|c|c|c|c|c|c|c|}
\hline Study Phase & Baseline & Randomisation & Surgery & & $\begin{array}{l}\text { atm } \\
\text { od ( }\end{array}$ & & & ow & $p(m$ & hs) \\
\hline Assessment & & & & 1 & 6 & 12 & 6 & 12 & 24 & 36 \\
\hline Study medication dispensed & & $\mathbf{x}$ & 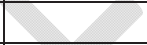 & & & & & & & \\
\hline Informed consent & $\mathbf{X}$ & & 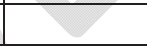 & & & & & & & \\
\hline Medical history & $\mathbf{x}$ & & & & & & & & & \\
\hline Weight & $\mathrm{X}$ & & $x$ & $\mathbf{x}$ & $\mathrm{x}$ & $x$ & $\mathrm{x}$ & $\mathrm{X}$ & $x$ & $\mathrm{x}$ \\
\hline Blood pressure & $\mathrm{x}$ & 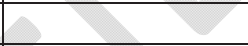 & $\mathbf{x}$ & $\mathbf{x}$ & $\mathbf{x}$ & $\mathbf{x}$ & $\mathbf{X}$ & $\mathbf{X}$ & $\mathbf{x}$ & $\mathbf{x}$ \\
\hline Demographics & $\mathrm{x}$ & - & & & & & & & & \\
\hline Physical exam & $\mathrm{X}$ & & & & & & & & & \\
\hline Dialysis details & $x$ & 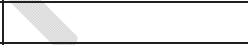 & & $\mathbf{x}$ & $\mathrm{x}$ & $\mathbf{x}$ & $x$ & $\mathbf{x}$ & $\mathbf{x}$ & $x$ \\
\hline $\begin{array}{l}\text { Description and status of } \\
\text { vascular access }\end{array}$ & $x$ & & $\mathbf{x}$ & $x$ & $\mathbf{x}$ & $\mathbf{x}$ & $\mathbf{x}$ & $\mathbf{X}$ & $\mathbf{x}$ & $\mathbf{x}$ \\
\hline Concomitant meds & $x$ & & $\mathbf{x}$ & $\mathbf{x}$ & $\mathbf{x}$ & $x$ & $x$ & & & \\
\hline Adverse events & $\mathrm{X}$ & & $x$ & 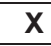 & $x$ & $x$ & $x$ & & & \\
\hline Fasting blood test & $x$ & & & & $\mathrm{x}$ & $\mathbf{x}$ & & & & \\
\hline
\end{tabular}

\section{Are there any risks?}

There is a large amount of research about the use of aspirin for prevention of blood clots. In general, aspirin is very good in stopping blood clots and there is only a small possibility of bleeding while taking aspirin. However, your doctor will keep a close eye on you and you should report any unusual bleeding immediately to your renal unit nurses or nephrologist. Symptoms that could have serious consequences include black stools, feeling faint, signs of allergic reactions and abdominal pain.

Fish oil is generally doesn't have many side effects. Gastrointestinal side effects such as nausea, vomiting, diarrhoea and non-specific discomfort are the most commonly reported. These problems can be reduced by taking the fish oil capsules with a glass of cold water. The combination of aspirin and fish oil should have a lower risk of bleeding than just taking aspirin. 
If you are a woman who is able to become pregnant, you should use an effective method of birth control for the three months that you are taking the study medication. If you do become pregnant, you should stop all study medications and inform your renal unit nurses or nephrologist immediately. The study medications are not known to affect men fathering children.

\section{Potential Benefits}

If you decide to take part in this trial, your medical condition will be very closely monitored. Any changes in your health will be very quickly identified.

The results from this study may help other haemodialysis patients having AVFs in the future.

\section{Confidentiality}

All information collected for this study will be treated with complete confidentiality. You will be asked to give permission to any regulatory authorities related to the study to have access your medical records. This information will only be used for purposes of this study. Information gathered by the ANZDATA Registry (the Australia and New Zealand Dialysis and Transplant Registry) may also be used as part of this study. Medical records will be stored for accessibility for at least 15 years.

\section{Costs}

Your involvement in this study will not cost you anything. You will not be paid to take part in this study. Taking part in this study will not change usual hospital liability arrangements and processes.

\section{Do you have a choice?}

Entry into this study is entirely voluntary. Choosing not to take part in this study will not affect your treatment or care in any way. The doctors will continue to treat you with the best means available.

If you agree to participate in this study you will be asked to sign a consent form. However you may withdraw from the study at any time without giving a reason. Your doctor may also decide that you should stop if they believe it is in your best interest. You will be told if any new information arises which might affect your decision to be in the study.

For further information about this study, please contact (medical contact, research nurse contact, phone numbers).

\section{Ethics Approval}

This study has been reviewed and approved by the (local) Ethics Committee. Should you wish to discuss the matter with someone not directly involved, you can contact (Ethics Committee contact, number).

This study has been cleared by one of the human ethics committees of the University of Queensland in accordance with the National Health and Medical Research Council's guidelines. You are of course, free to discuss your participation in this study with project staff (contactable on .......................). If you would like to speak to an officer of the University not involved in the study, you may contact the Ethics Officer on 0733653924. 


\subsection{Sample Patient Consent Form}

(Name of institution)

\section{Consent Form}

\section{A randomised, double-blind, placebo-controlled, factorial-design trial to assess the effect of aspirin and fish oil (omega- 3 fatty acids) in the prevention of early thrombosis in arterio-venous fistulae in patients with Stage IV or V chronic kidney disease requiring haemodialysis}

- FAVOURED Study (Fish oil and Aspirin in Vascular access OUtcomes In REnal Disease

- I agree to participate in the above named clinical trial.

- I have read and understood and kept a copy of the Patient Information Sheet.

- I acknowledge and understand the methods and demands relating to the study and the possible risks and inconveniences which may occur.

- I have had my questions answered to my satisfaction.

- I understand that I may withdraw from the study at any time without giving any reason and that this will not affect my medical treatment.

- I give my permission for access to my medical records including information gathered by the ANZDATA Registry (the Australia and New Zealand Dialysis and Transplant Registry) for the purposes of this research.

- I give my permission for access to my medical records for the purposes of this research, including the pre-surgical Doppler vein mapping.

\begin{tabular}{lcc}
\hline Patient's signature & Printed name & $\begin{array}{c}\text { Date } \\
\text { (to be personally dated) }\end{array}$ \\
\hline Witness signature & Printed name & Date \\
& (to be personally dated) \\
I have explained the nature and purpose of this study to the above participant and have \\
answered their questions.
\end{tabular}

Investigator's signature

Printed name

Date

AKTN Protocol - FAVOURED Study

Version 9 30/11/2010 


\subsection{Classification of Chronic Kidney Disease}

The Kidney Disease Outcomes Quality Initiative (K/DOQI) of the National Kidney Foundation (NKF) defines chronic kidney disease (CKD) as either kidney damage or a decreased kidney glomerular filtration rate (GFR) of $<60 \mathrm{~mL} / \mathrm{min} / 1.73 \mathrm{~m}^{2}$ for 3 or more months. February $2002 \mathrm{~K} / \mathrm{DOQ}$ classification of the stages of CKD:

- Stage 1: Kidney damage with normal or increased GFR (>90 mL/min/1.73 $\left.\mathrm{m}^{2}\right)$

- Stage 2: Mild reduction in GFR $\left(60-89 \mathrm{~mL} / \mathrm{min} / 1.73 \mathrm{~m}^{2}\right)$

- Stage 3: Moderate reduction in GFR $\left(30-59 \mathrm{~mL} / \mathrm{min} / 1.73 \mathrm{~m}^{2}\right)$

- Stage 4: Severe reduction in GFR $\left(15-29 \mathrm{~mL} / \mathrm{min} / 1.73 \mathrm{~m}^{2}\right)$

- Stage 5: Kidney failure (GFR $<15 \mathrm{~mL} / \mathrm{min} / 1.73 \mathrm{~m}^{2}$ or dialysis) 
13.5 Lifestyle questionnaire

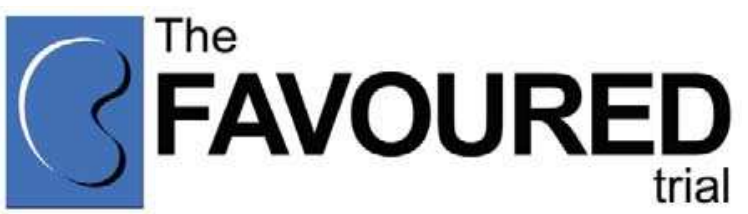

\section{LIFESTYLE QUESTIONNAIRE}

\section{Fish Oil and Aspirin in the Prevention of Thrombosis in Chronic Kidney Disease}

Thank you for your interest in participating in this research trial. We would very much appreciate your help in filling in this questionnaire, which will give us information about your lifestyle, including diet and exercise. Once you have completed the questionnaire please return it to the supervising research staff member.

The questionnaire should take approximately 15 minutes to complete.

Please take your time and answer all questions as accurately as possible.

\section{N.B. ALL INFORMATION WILL REMAIN STRICTLY CONFIDENTIAL}

\begin{tabular}{|l|l}
\hline Today's Date $(\mathrm{dd} / \mathrm{mm} / \mathrm{yyyy})$ \\
\hline Patient's Initials \\
\hline Date of Birth $(\mathrm{dd} / \mathrm{mm} / \mathrm{yyyy})$ \\
\hline
\end{tabular}




\section{YOUR DRINKING DETAILS}

1. Do you drink alcohol? (Circle one)

I have never drunk alcohol (go to Question 4) 1

I used to drink alcohol (go to Question 2) . . . . 2

I occasionally drink alcohol (go to Question 3) . . . 3

2. If you gave up drinking alcohol, how long ago did you stop? (Circle one)

Less than 3 months ago . $\quad . \quad$. $\quad$. $\quad$. 1

3 - 6 months ago . $\quad . \quad$. $\quad . \quad$. $\quad . \quad 2$

More than 6 months but less than 1 year ago . . 3

More than 1 year ago . . . . . . . . 4

(go to Question 4)

3. How many standard drinks (see below) of alcohol do you usually drink? (Circle one)

\begin{tabular}{|l|c|c|c|c|c|c|}
\hline & $\begin{array}{c}\text { Less than 1 } \\
\text { per week }\end{array}$ & $\begin{array}{c}1-6 \text { per } \\
\text { week }\end{array}$ & 1 per day & $2-3$ per day & 4 per day & $\begin{array}{c}\text { More than 4 } \\
\text { per day }\end{array}$ \\
\hline A. Beer & 1 & 2 & 3 & 4 & 5 & 6 \\
\hline B. Wine & 1 & 2 & 3 & 4 & 5 & 6 \\
\hline C. Spirits & 1 & 2 & 3 & 4 & 5 & 6 \\
\hline
\end{tabular}

\section{DO YOU KNOW A STANDARD DRINK WHEN YOU SEE ONE?}

To help you keep count of your drinks, remember any drink containing about 10 grams of alcohol is called a standard drink. For example:

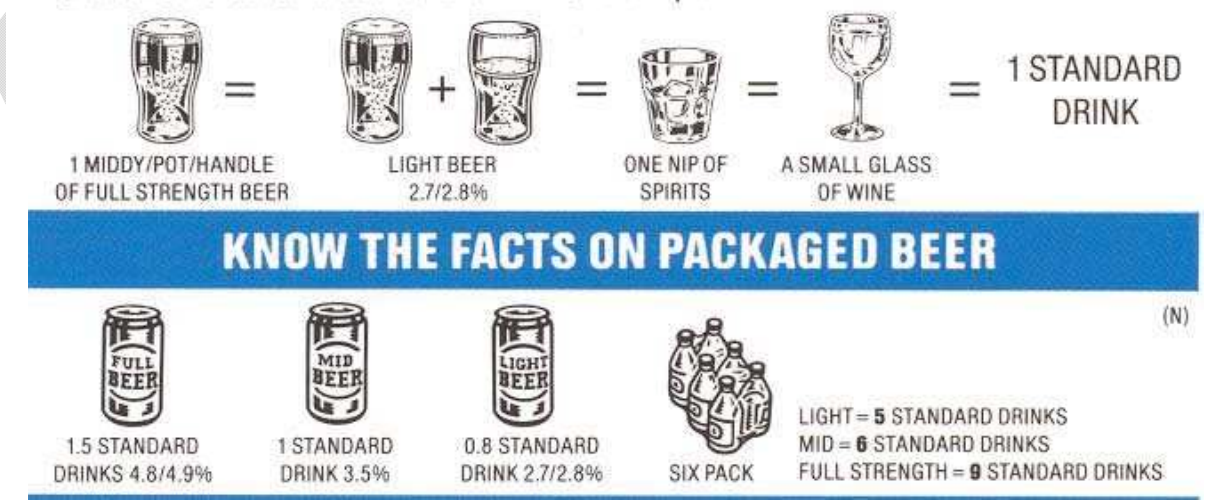




\section{YOUR SMOKING HISTORY}

4. Do you smoke cigarettes, cigars, pipes or other tobacco products? (Circle one)

I have never smoked (go to Question 7) $\quad$. $\quad . \quad$. $\quad 1$

I used to smoke (go to Question 5) . $\quad$. $\quad . \quad$. $\quad$. 2

I am currently a smoker (go to Question 6) . . . . 3

5. If you gave up smoking, about how long ago did you stop? (Circle one)

Less than 3 months ago $\quad$. $\quad$. $\quad$. $\quad$. $\quad$. $\quad$. 1

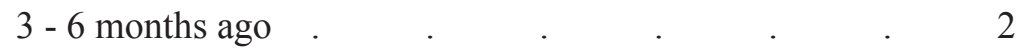

More than 6 months but less than 1 year ago . . $\quad 3$

More than 1 year ago . . . . . . . $4 \quad 4$

(go to Question 7)

6. If you smoke, how many cigarettes, cigars, etc do you smoke per day on average?

per day

\section{YOUR LEVEL OF PHYSICAL ACTIVITY}

7. How often do you exercise (includes taking walks, gardening or playing sport)? (Circle one)

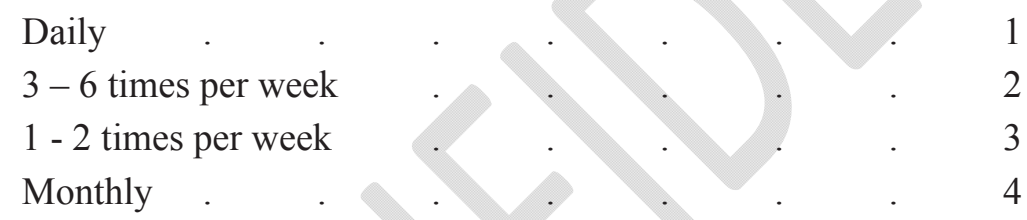

8. How long, on average, are you active for on these days? $\quad$ min per day

\section{YOUR DIET}

9. How many serves of vegetables or salad do you eat per day on average? (A serve is half-a cup)

per day

10. How many serves of fruit do you eat per day on average? (A serve is half-a cup or the equivalent of one medium apple)

per day

11. How many times per week do you eat fried food?

per week

12. How many times per week do you eat pies, cakes, biscuits or per week chocolate? 
13. How often do you eat fish on average? (Circle one)

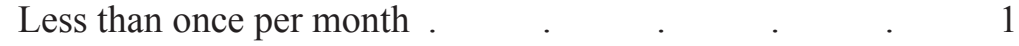

1 - 3 times per month . . . . . . . . 2

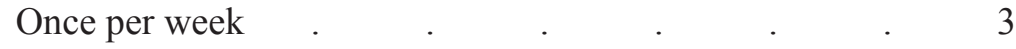

2 - 4 times per week $\quad . \quad$. $\quad . \quad$. $\quad . \quad . \quad 4 \quad 4$

More than 5 times per week $\quad$. $\quad$. $\quad$. $\quad$. 5

14. What type of fish do you usually eat? (Circle one)

Fish, grilled, steamed, or baked . . . . . . . . 1

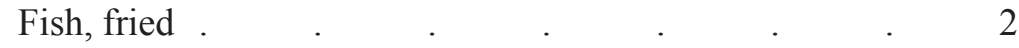

Fish, canned (include tuna, salmon, sardines, mackerel) . $\quad 3$

Shellfish (include prawns, scallops, crayfish) . . . 4

15. If you have recently taken fish oil capsules, on average, how many did you take?

per day

16. How often do you eat red meat on average? (Circle one)

Less than once per month . . . . . . . . . 1

1 - 3 times per month . . . . . . . . . . 2

Once per week . . . . . . . .

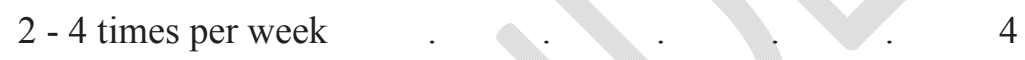

More than 5 times per week $\quad . \quad$. $\quad . \quad$. 5

17. When you red eat meat do you remove the fat? (Circle one)

Usually . . . . . . . . . . . . . 1

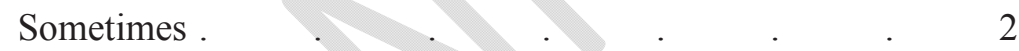

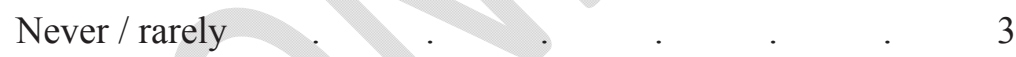

18 Do you add salt to food at the table? (Circle one)

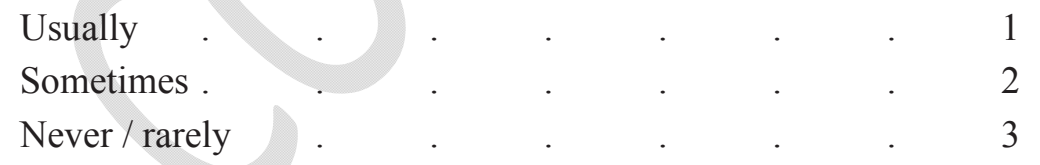

19. How often do you eat chicken on average? (Circle one)

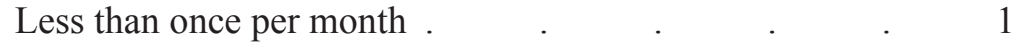

1 - 3 times per month . . . . . . . 2

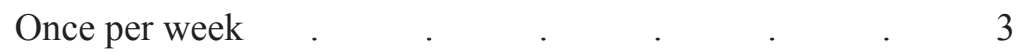

2 - 4 times per week $\quad . \quad$. $\quad . \quad$. $\quad . \quad$. 4

More than 5 times per week $\quad$. $\quad$. $\quad$. 5 
20. When you eat chicken do you remove the skin? (Circle one)

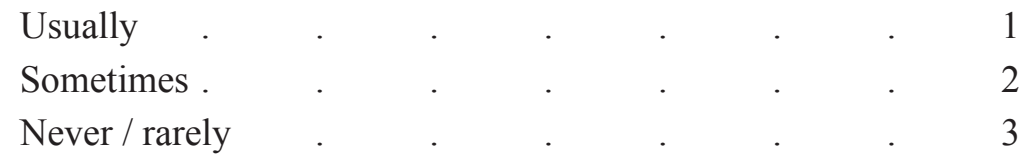

21. What kind of milk do you usually use? (Circle one)

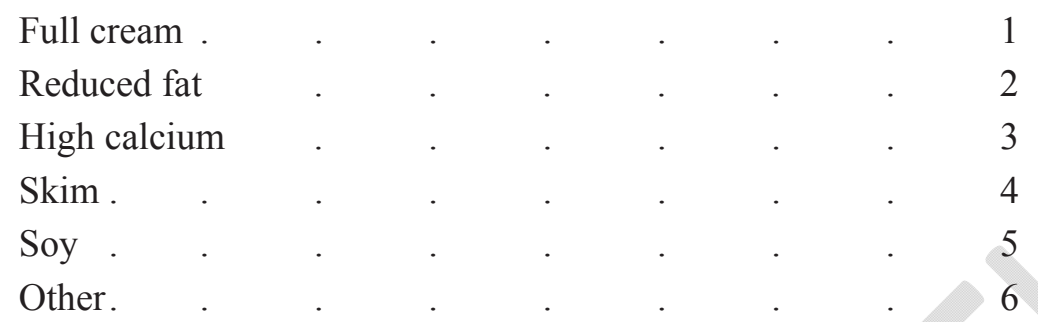

If other, please specify

22. How much milk do you consume per day?

1 cup of coffee or tea has the equivalent of 1 tablespoon of milk

1 bowl of cereal has the equivalent of $1 / 2$ cup of milk tablespoons per day

cups per day

\section{YOUR FAMILY HISTORY}

23. Did your father have cardiovascular disease (stroke or other disease affecting the heart or blood vessels) before he was 45 years old? (Circle one)

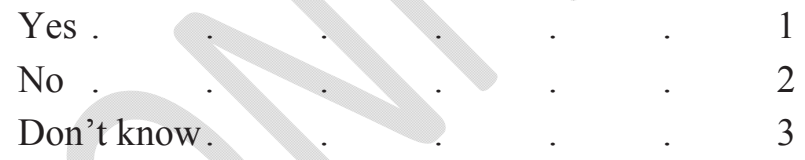

24. Did your mother have cardiovascular disease before she was 55 years old? (Circle one)

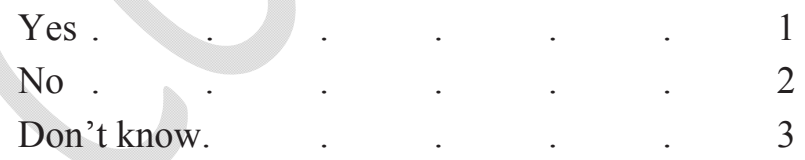

\section{THANK YOU FOR YOUR CO-OPERATION}




\subsection{Data related to patency rates}

Table: Studies comparing long-term patency AVF versus AVG.

\begin{tabular}{|c|c|c|c|c|c|c|c|c|c|c|}
\hline Study & $\begin{array}{l}\text { Study } \\
\text { Type }\end{array}$ & Country & $\begin{array}{c}\text { Access } \\
\text { Type }\end{array}$ & Number & $\begin{array}{c}1^{\circ} \text { Failure } \\
(\%)\end{array}$ & $\begin{array}{c}1^{\circ} \text { Patency } \\
1 \text { yr }(\%)\end{array}$ & $\begin{array}{c}1^{\circ} \text { Patency } \\
2 y r(\%)\end{array}$ & $\begin{array}{c}1^{\circ} \text { Patency } \\
3 y r(\%)\end{array}$ & $\begin{array}{l}1^{\circ} \text { failure } \\
\text { included }\end{array}$ & $\begin{array}{c}\text { Intervention } \\
\text { Rate } \\
\text { (/acc-yr) }\end{array}$ \\
\hline \multirow{2}{*}{ Palder 1985} & \multirow{2}{*}{$\mathrm{R}, \mathrm{S}$} & \multirow{2}{*}{ USA } & RCF & 154 & 24 & $60^{*}$ & $50^{*}$ & $42^{*}$ & $\mathrm{Y}$ & $\star \star$ \\
\hline & & & AVG & 163 & NS & $78^{*}$ & $70^{*}$ & $56^{\star}$ & $Y$ & ** \\
\hline \multirow{2}{*}{ Winsett 1985} & \multirow{2}{*}{$\mathrm{R}, \mathrm{S}$} & \multirow{2}{*}{ USA } & RCF & 273 & 27 & & & $72\left(84^{\star}\right)$ & $\mathrm{N}$ & 0.06 \\
\hline & & & AVG & 202 & NS & & & $39\left(70^{*}\right)$ & $\mathrm{N}$ & 0.44 \\
\hline \multirow{2}{*}{ Kherlakian 1986} & \multirow{2}{*}{$\mathrm{R}, \mathrm{S}$} & \multirow{2}{*}{ USA } & RCF & 100 & 12 & $71^{*}$ & $66^{*}$ & $64^{*}$ & $\mathrm{Y}$ & $\star \star \star *$ \\
\hline & & & AVG & 100 & 4 & $75^{\star}$ & $61^{*}$ & $50^{*}$ & $Y$ & $\star \star \star$ \\
\hline \multirow{2}{*}{ Zibari 1988} & \multirow{2}{*}{$\mathrm{R}, \mathrm{S}$} & \multirow{2}{*}{ USA } & AVF & 160 & & & & & & 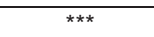 \\
\hline & & & AVG & 166 & & & & & & *** \\
\hline \multirow{2}{*}{ Coburn 1994} & \multirow{2}{*}{$\mathrm{R}, \mathrm{S}$} & \multirow{2}{*}{ USA } & Basilic & 59 & NS & $90\left(90^{\star}\right)$ & $86\left(86^{\star}\right)$ & & NS & 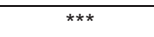 \\
\hline & & & AVG & 47 & NS & $70\left(87^{\star}\right)$ & $49\left(64^{\star}\right)$ & & NS & 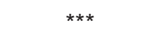 \\
\hline \multirow{2}{*}{ Burger 1995} & \multirow{2}{*}{$\mathrm{P}, \mathrm{S}^{\wedge}$} & \multirow{2}{*}{ Netherlands } & $\mathrm{RCF}$ & 208 & NS & $53\left(79^{*}\right)$ & $36\left(68^{\star}\right)$ & $24\left(59^{\star}\right)$ & NS & NS \\
\hline & & & AVG & 46 & NS & $37\left(61^{*}\right)$ & $21\left(60^{*}\right)$ & $17\left(54^{\star}\right)$ & NS & NS \\
\hline \multirow{2}{*}{ Enzler 1996} & \multirow{2}{*}{$\mathrm{R}, \mathrm{S}$} & \multirow{2}{*}{ Switzerland } & RCF & 429 & NS & $70\left(74^{*}\right)$ & $61\left(67^{\star}\right)$ & $59\left(64^{*}\right)$ & NS & $\star *$ \\
\hline & & & AVG & 69 & NS & $41\left(58^{*}\right)$ & $24\left(47^{\star}\right)$ & $24\left(40^{\star}\right)$ & NS & $\star \star \star$ \\
\hline \multirow{2}{*}{ Rocco 1996} & \multirow{2}{*}{$\mathrm{R}, \mathrm{S}$} & \multirow{2}{*}{ USA } & RCF & 48 & 31 & $55\left(78^{\star}\right)$ & & & NS & 0.34 \\
\hline & & & AVG & 40 & 12 & $60\left(88^{*}\right)$ & & & NS & 0.8 \\
\hline \multirow{2}{*}{ Miller 1997} & \multirow{2}{*}{$\mathrm{R}, \mathrm{S}$} & \multirow{2}{*}{ USA } & AVF & 75 & NS & $84\left(83^{*}\right)$ & $74\left(70^{*}\right)$ & & NS & 0.17 \\
\hline & & & AVG & 23 & NS & $47\left(73^{*}\right)$ & $38\left(58^{*}\right)$ & & NS & 1.17 \\
\hline \multirow{2}{*}{ Hodges 1997} & \multirow{2}{*}{$\mathrm{R}, \mathrm{S}$} & HCA & AVF & 87 & 31 & $43\left(46^{\star}\right)$ & & & $\bar{Y}$ & 0.07 \\
\hline & & USA & AVG & 236 & NS & $41\left(59^{*}\right)$ & & & $Y$ & 0.5 \\
\hline & & & AVF & 108 & 8 & 83 & & & $Y$ & NS \\
\hline Silva 1998 & $\mathrm{P}, \mathrm{S}$ & USA & AVG & 52 & 8 & 74 & & & $Y$ & NS \\
\hline Mloum 1000 & & $H C A$ & Basilic & 30 & NS & & $70\left(70^{\star}\right)$ & & NS & $\star \star *$ \\
\hline Matsuura 1998 & $\mathrm{R}, \mathrm{S}$ & USA & AVG & 68 & NS & & $46\left(51^{*}\right)$ & & NS & $\star \star *$ \\
\hline & & & AVF & 235 & 12 & & $54\left(70^{\star}\right)$ & & $\bar{Y}$ & $\star \star *$ \\
\hline Kalman 1999 & P,S & Canada & AVG & 231 & NS & & $18\left(60^{\star}\right)$ & & $Y$ & ** \\
\hline Anhornon & $R_{0}$ & & AVF & 99 & 18 & 84 & & & $\mathrm{~N}$ & 0.07 \\
\hline Ascher 2000 & $\mathrm{R}, \mathrm{S}$ & USA & AVG & 122 & NS & 54 & & & $\mathrm{~N}$ & 0.74 \\
\hline
\end{tabular}

AKTN Protocol - FAVOURED Study

Version 9 30/11/2010

Page 48 of 50 


\begin{tabular}{|c|c|c|c|c|c|c|c|c|c|c|}
\hline Study & $\begin{array}{l}\text { Study } \\
\text { Type }\end{array}$ & Country & $\begin{array}{l}\text { Access } \\
\text { Type }\end{array}$ & Number & $\begin{array}{c}1^{\circ} \text { Failure } \\
(\%)\end{array}$ & $\begin{array}{c}1^{\circ} \text { Patency } \\
1 \mathrm{yr}(\%)\end{array}$ & $\begin{array}{c}1^{\circ} \text { Patency } \\
2 \mathrm{yr}(\%)\end{array}$ & $\begin{array}{c}1^{\circ} \text { Patency } \\
\text { 3yr (\%) }\end{array}$ & $\begin{array}{l}1^{\circ} \text { failure } \\
\text { included }\end{array}$ & $\begin{array}{c}\text { Intervention } \\
\text { Rate } \\
\text { (acc/yr) }\end{array}$ \\
\hline \multirow{2}{*}{ Staramos $2000^{+}$} & \multirow{2}{*}{$\mathrm{R}, \mathrm{S}$} & \multirow{2}{*}{ Greece } & AVF & 68 & NS & $67\left(67^{\star}\right)$ & $52\left(52^{\star}\right)$ & $44\left(44^{\star}\right)$ & $Y$ & NS \\
\hline & & & AVG & 67 & NS & $66\left(81^{\star}\right)$ & $53\left(65^{\star}\right)$ & $38\left(58^{\star}\right)$ & $Y$ & NS \\
\hline \multirow{3}{*}{ Gibson 2001b } & \multirow{3}{*}{$\mathrm{P}, \mathrm{M}$} & \multirow{3}{*}{ USA } & AVF & 492 & NS & $56\left(73^{\star}\right)$ & $40\left(64^{\star}\right)$ & & NS & $1.0^{c}$ \\
\hline & & & VTAVF & 181 & NS & $44\left(68^{\star}\right)$ & $28\left(60^{\star}\right)$ & & NS & $1.32^{c}$ \\
\hline & & & AVG & 1574 & NS & $38\left(72^{\star}\right)$ & $25\left(60^{\star}\right)$ & & NS & $1.91^{c}$ \\
\hline \multirow{2}{*}{ Allon 2001} & \multirow{2}{*}{$\mathrm{R}, \mathrm{S}$} & \multirow{2}{*}{ USA } & AVF & 139 & 46 & $42\left(44^{\star}\right)$ & & & $Y$ & 0.57 \\
\hline & & & AVG & 78 & 21 & $43\left(48^{\star}\right)$ & & & $Y$ & 1.67 \\
\hline \multirow{2}{*}{ Gibson 2001a } & \multirow{2}{*}{$\mathrm{R}, \mathrm{S}$} & \multirow{2}{*}{ USA } & AVF & 130 & 23 & $56\left(72^{\star}\right)$ & $41\left(61^{\star}\right)$ & & $Y$ & $\star \star$ \\
\hline & & & AVG & 92 & NS & $36\left(58^{\star}\right)$ & $11\left(41^{*}\right)$ & & $Y$ & ** \\
\hline \multirow{3}{*}{ Oliver 2001} & \multirow{3}{*}{$\mathrm{R}, \mathrm{S}$} & \multirow{3}{*}{ USA } & $\mathrm{BCF}$ & 56 & 32 & 64 & & & $\mathrm{~N}$ & 0.4 \\
\hline & & & Basilic & 59 & 21 & 64 & & & $\mathrm{~N}$ & 0.7 \\
\hline & & & AVG & 80 & 15 & 62 & 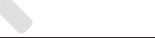 & & $\mathrm{N}$ & 2.4 \\
\hline \multirow{3}{*}{ Dixon 2002} & \multirow{3}{*}{$\mathrm{R}, \mathrm{S}$} & \multirow{3}{*}{ USA } & RCF & 88 & 32 & $44\left(52^{*}\right)$ & $40\left(48^{\star}\right)$ & $31\left(43^{\star}\right)$ & $Y$ & 1.4 \\
\hline & & & BCF & 117 & 28 & $62\left(69^{*}\right)$ & $48\left(59^{\star}\right)$ & $37\left(53^{*}\right)$ & $Y$ & 2.71 \\
\hline & & & AVG & 117 & 22.6 & $27\left(54^{*}\right)$ & $13\left(42^{\star}\right)$ & $7\left(31^{\star}\right)$ & $Y$ & 3.84 \\
\hline \multirow{2}{*}{ Pisoni 2002} & \multirow{2}{*}{$\mathrm{P}, \mathrm{M}$} & \multirow{2}{*}{ USA } & AVF & 177 & NS & 68 & & & $\mathrm{~N}$ & NS \\
\hline & & & AVG & 251 & NS & 49 & & & $\mathrm{~N}$ & NS \\
\hline \multirow{2}{*}{ Lawrence 2002} & \multirow{2}{*}{$\mathrm{R}, \mathrm{S}$} & \multirow{2}{*}{ Australia } & AVF & 64 & NS & 87 & 87 & & NS & NS \\
\hline & & & AVG & 26 & NS & 63 & 50 & & NS & NS \\
\hline \multirow{4}{*}{ Shenoy 2003} & \multirow{4}{*}{$\mathrm{R}, \mathrm{M}$} & \multirow{4}{*}{ USA } & AVF $^{\#}$ & 242 & 17 & & $54\left(67^{\star}\right)$ & & $Y$ & 0.22 \\
\hline & & & AVG ${ }^{\#}$ & 440 & 17 & & $36\left(39^{\star}\right)$ & & $Y$ & 0.86 \\
\hline & & & AVF \#\# & 276 & 28 & & $34\left(48^{\star}\right)$ & & $Y$ & 0.37 \\
\hline & & & AVG $\# \#$ & 384 & 28 & & $17\left(19^{\star}\right)$ & & $Y$ & 1.73 \\
\hline \multirow{2}{*}{ Fischer 2003} & \multirow{2}{*}{$\mathrm{R}, \mathrm{S}$} & Auctrolin & RCF & 147 & NS & $69^{*}$ & $63^{*}$ & $55^{*}$ & NS & $\star *$ \\
\hline & & Australla & AVG & 50 & NS & $89^{*}$ & $75^{\star}$ & $68^{*}$ & NS & $\star *$ \\
\hline & & & BCF & 30 & 27 & $53\left(67^{\star}\right)$ & $34\left(56^{\star}\right)$ & & $\mathrm{Y}$ & ** \\
\hline Choi 2003 & $\mathrm{R}, \mathrm{S}$ & USA & Basilic & 42 & 5 & $76\left(83^{*}\right)$ & $68\left(75^{\star}\right)$ & & $Y$ & ** \\
\hline & & & AVG & 53 & NS & $47\left(59^{*}\right)$ & $26\left(40^{*}\right)$ & & $Y$ & ** \\
\hline
\end{tabular}

AKTN Protocol - FAVOURED Study

Version 9 30/11/2010

Page 49 of 50 


\begin{tabular}{|c|c|c|c|c|c|c|c|c|c|c|}
\hline Study & $\begin{array}{l}\text { Study } \\
\text { Type }\end{array}$ & Country & $\begin{array}{c}\text { Access } \\
\text { Type }\end{array}$ & Number & $\begin{array}{c}\text { 10 Failure } \\
\text { (\%) }\end{array}$ & $\begin{array}{c}\text { 10 Patency } \\
1 \mathrm{yr}(\%)\end{array}$ & $\begin{array}{c}\text { 10 Patency } \\
2 \mathrm{yr}(\%)\end{array}$ & $\begin{array}{c}\text { 10 Patency } \\
\text { 3yr (\%) }\end{array}$ & $\begin{array}{l}\text { 1o failure } \\
\text { included }\end{array}$ & $\begin{array}{c}\text { Intervention } \\
\text { Rate } \\
\text { (acc/yr) }\end{array}$ \\
\hline \multirow[b]{2}{*}{ Huber 2003} & \multirow{2}{*}{ Meta } & \multirow[b]{2}{*}{ NA } & AVF \#\#\# & 1849 & NS & $72^{\mathrm{a}}\left(86^{\star}\right)$ & $51^{\mathrm{b}}\left(77^{\star}\right)$ & & NS & NS \\
\hline & & & AVG & 1245 & NS & $58^{\mathrm{a}}\left(76^{\star}\right)$ & $33^{b}\left(55^{\star}\right)$ & & NS & NS \\
\hline \multirow{2}{*}{ Perara 2004} & \multirow{2}{*}{$\mathrm{R}, \mathrm{S}$} & \multirow{2}{*}{ USA } & AVF & 100 & $11 \%$ & $56\left(64^{\star}\right)$ & $39\left(53^{\star}\right)$ & & $Y$ & 0.53 \\
\hline & & & AVG & 131 & NS & $36\left(65^{\star}\right)$ & $9\left(46^{\star}\right)$ & & $\mathrm{Y}$ & 0.92 \\
\hline \multirow{4}{*}{ Kawecka 2005} & \multirow{4}{*}{$\mathrm{R}, \mathrm{S}$} & \multirow{4}{*}{ Poland } & $\mathrm{RCF}$ & 540 & NS & $52\left(67^{\star}\right)$ & $41\left(56^{\star}\right)$ & & NS & NS \\
\hline & & & $\mathrm{BCF}$ & 143 & NS & $45\left(61^{*}\right)$ & $31\left(44^{\star}\right)$ & & NS & NS \\
\hline & & & Basilic & 85 & NS & $42\left(54^{\star}\right)$ & $28\left(38^{\star}\right)$ & & NS & NS \\
\hline & & & AVG & 86 & NS & $48\left(56^{\star}\right)$ & $21\left(33^{*}\right)$ & & NS & NS \\
\hline \multirow{2}{*}{ Fitzgerald 2005} & \multirow{2}{*}{$\mathrm{R}, \mathrm{S}$} & \multirow{2}{*}{ USA } & UAAVF & 86 & NS & 50 & 46 & & NS & ** \\
\hline & & & $A V G$ & 60 & NS & 50 & 37 & & NS & ** \\
\hline
\end{tabular}

Table from Polkinghorne, unpublished

\section{Footnotes:}

$1^{\circ}=$ Primary

$\mathrm{AVF}=$ native arteriovenous fistula

$\mathrm{RCF}=$ Radiocephalic AVF

$\mathrm{BCF}=$ Brachiocephalic AVF

Basilic $=$ Brachiobasilic AVF

UAAVF $=$ Upper Arm AVF (either BCF or Brachiobasilic AVF)

$\mathrm{AVG}=$ arteriovenous (PTFE) graft

VTAVF $=$ Venous transposition AVF

$\mathrm{R}=\quad$ Retrospective

$\mathrm{P}=\quad$ Prospective

$\mathrm{S}=\quad$ Single centre

$\mathrm{M}=$ multicentre

Meta $=$ meta-analysis

$\mathrm{Y}=\quad$ Yes
$\mathrm{N}=\mathrm{No}$

NS $=\quad$ Not stated

$\mathrm{NA}=\quad$ Not applicable

* Indicates secondary patency,

** Overall rates not given however significantly more thrombectomies and revisions in the AVG group

** Overall rates not given however significantly more complications seen in the AVG group $\wedge$ Retrospective 1971-1980, prospective 1980-1991

\# Clips used

\#\# Sutures used

\#\#\# Upper arm AVF only + only patients 70 years and over

6 month patency

${ }^{\mathrm{b}} 18$ month patency

${ }^{\circ}$ Incident Rate Ratio, AVF comparison group VTAVF vs AVG $p=0.04$, AVG vs AVF $p<0.01$

AKTN Protocol - FAVOURED Study

Version 9 30/11/2010

Page 50 of 50 


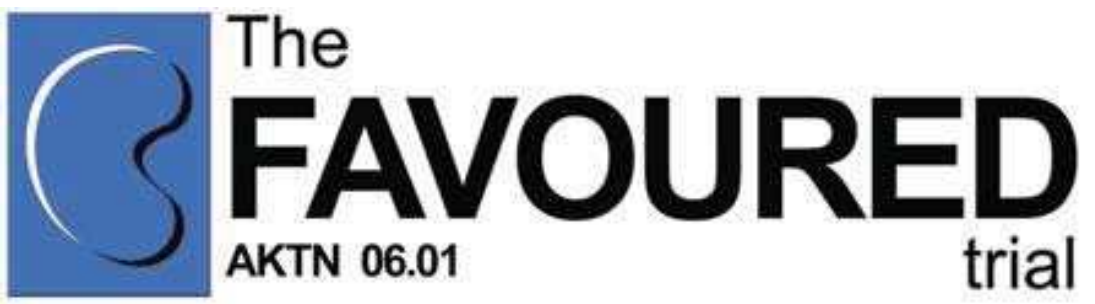

\section{STATISTICAL ANALYSIS PLAN (Version 1.11)}

Protocol Title: $\quad$ A randomised, double-blind, placebo-controlled factorialdesign trial to assess the effect of aspirin and fish oil (omega3 fatty acids) in the prevention of early thrombosis in arteriovenous fistulae in patients with stage IV or V chronic kidney disease requiring haemodialysis (Version 9)

Short title: $\quad$ FAVOURED (Fish oil and Aspirin in Vascular access OUtcomes in REnal Disease).

Protocol Date: $\quad 30$ November 2010

Trial Registration: Australian New Zealand Clinical Trials Registry number ACTRN12607000569404

\section{Australasian Kidney Trials Network}

Princess Alexandra Hospital, 199 Ipswich Road, Woolloongabba QLD 4102 


\section{DOCUMENT HISTORY}

\begin{tabular}{|l|l|l|}
\hline \multicolumn{1}{|c|}{ Version } & \multicolumn{1}{|c|}{ Reason(s) for change } & Date \\
\hline V1.11 & Initial document & 2 September 2015 \\
\hline & & \\
\hline
\end{tabular}

\section{APPROVALS}

Principal Investigator \& Chair of Trial Management Committee

\begin{tabular}{|l|c|c|}
\hline \multicolumn{1}{|c|}{ Name } & Signature & Date \\
\hline Prof Ashley Irish & & 2 September 2015 \\
\hline
\end{tabular}

Statistician, Australasian Kidney Trials Network

\begin{tabular}{|c|c|c|}
\hline \multicolumn{1}{|c|}{ Name } & Signature & Date \\
\hline Ms Elaine Pascoe & l'cascal & 2 September 2015 \\
\hline
\end{tabular}

Chair, Executive Operations Secretariat, Australasian Kidney Trials Network

\begin{tabular}{|c|c|c|}
\hline Name & Signature & Date \\
\hline A/Prof Carmel Hawley & Cucteuly & 2 September 2015 \\
\hline
\end{tabular}

This Statistical Analysis Plan is the property of the University of Queensland through the Australasian Kidney Trials Network. No section of this document may be copied, published, or used for any purpose without appropriate acknowledgement and the prior written permission of the Australasian Kidney Trials Network. 


\section{PREFACE}

This Statistical Analysis Plan (SAP) describes the planned analyses and reporting for the Australasian Kidney Trials Network (AKTN) trial number 06.01, "A randomised, doubleblind, placebo-controlled factorial-design trial to assess the effect of aspirin and fish oil (omega-3 fatty acids) in the prevention of early thrombosis in arterio-venous fistulae in patients with stage IV or V chronic kidney disease requiring haemodialysis: The FAVOURED Study". Version nine of the protocol details revisions to the design and primary outcome and, while the trial continued to be referred to as a two-by-two factorial design, the revised primary objective focussed solely on omega-3 PUFAs and the trial design associated with this objective is two parallel groups (omega-3 PUFAs versus matching placebo) stratified by aspirin use (no aspirin, randomised to aspirin, open-label aspirin) (1).

The structure and content of this SAP provide sufficient detail to meet the requirements identified by the International Conference on Harmonisation of Technical Requirements for Registration of Pharmaceuticals for Human Use (ICH): Guidance on Statistical Principles in Clinical Trials (2). All work planned and reported for this SAP will follow national and international guidelines for statistical practice (3-6).

The planned analyses identified in this SAP will be included in future manuscripts. Exploratory analyses not necessarily identified in this SAP may be performed to support planned analyses. Any post-hoc or unplanned analyses not specified in this SAP will be clearly identified as such in the Final Statistical Report (FSR) and manuscripts for publication.

This SAP was written by clinical investigators and statisticians and other trial staff who were blinded to treatment allocation and treatment-related study results and will remain so until the central database is locked and the final data are extracted for analysis. To ensure blinding, treatment allocations are stored in a separate location accessible only by the designated unblinded AKTN statistician.

The following documents were reviewed when preparing this SAP:

- Clinical Research Protocol for AKTN Trial Number 06.01 (7).

- Case report forms (CRFs) for AKTN Trial Number 06.01.

- Charter for the Data Safety Monitoring Board (DSMB) for the FAVOURED trial (8).

- ICH Guidance on Statistical Principles for Clinical Trials (2).

- ICH Guidance on Structure and Content of Clinical Study Reports (9).

Readers of this SAP are encouraged to read the Clinical Research Protocol for details on the conduct of this study and the timing of clinical assessments. 


\section{ABBREVIATIONS}

ABBREVIATION DEFINITION

$\begin{array}{ll}\text { ADR } & \text { Adverse Drug Reaction } \\ \text { AKTN } & \text { Australasian Kidney Trials Network } \\ \text { AVF } & \text { Arteriovenous Fistula } \\ \text { AVG } & \text { Arteriovenous Graft } \\ \text { CAP } & \text { Cannulation Assessment Period } \\ \text { CKD } & \text { Chronic Kidney Disease } \\ \text { CVC } & \text { Central Venous Catheter } \\ \text { DSMB } & \text { Data and Safety Monitoring Board } \\ \text { ESKD } & \text { End Stage Kidney Disease } \\ \text { FSR } & \text { Final Statistical Report } \\ \text { HD } & \text { Haemodialysis } \\ \text { ICH } & \text { International Conference on Harmonisation } \\ \text { ITT } & \text { Intention-To-Treat } \\ \text { NSAID } & \text { Non-steroidal anti-inflammatory drug } \\ \text { Omega-3 PUFAs } & \text { Omega-3 Polyunsaturated Fatty Acids } \\ \text { SAE } & \text { Serious Adverse Event } \\ \text { SAP } & \text { Statistical Analysis Plan } \\ \text { TMC } & \text { Trial Management Committee } \\ \text { WHO } & \text { World Health Organisation } \\ \end{array}$




\section{TABLE OF CONTENTS}

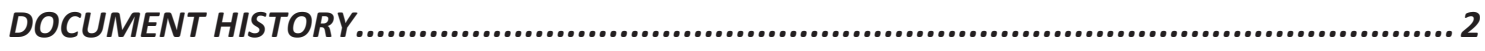

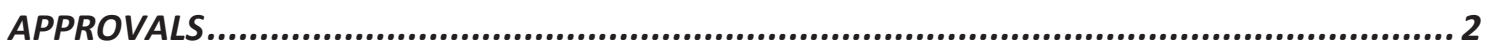

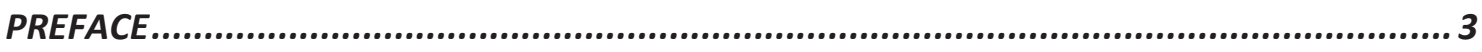

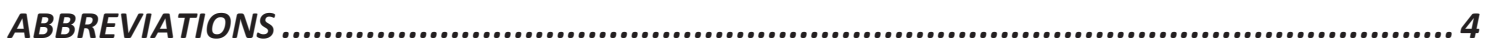

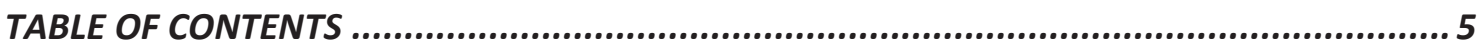

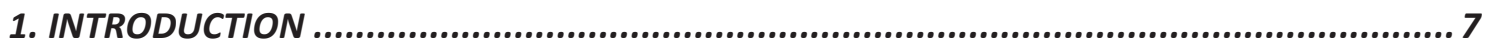

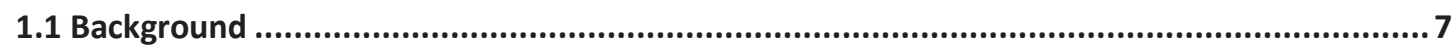

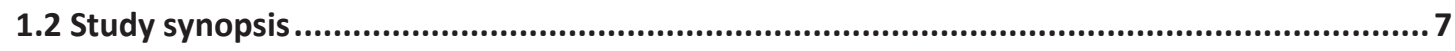

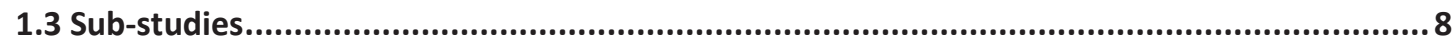

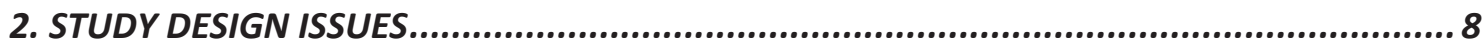

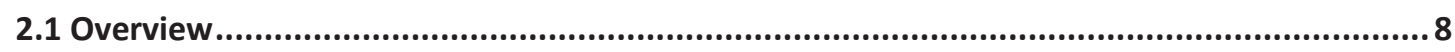

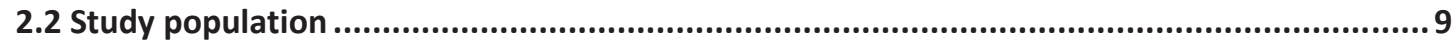

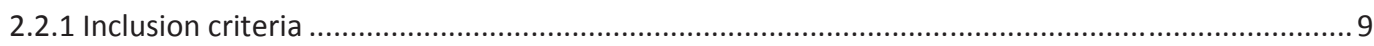

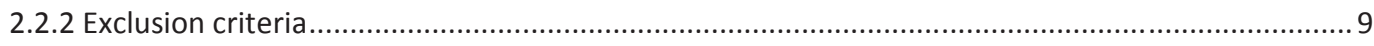

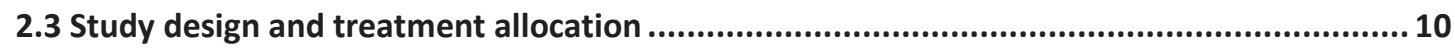

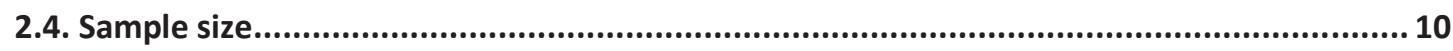

2.5 Treatment blinding and allocation concealment ........................................................... 11

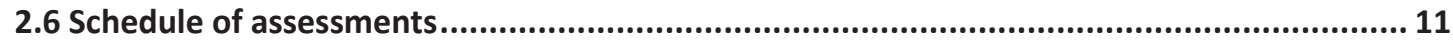

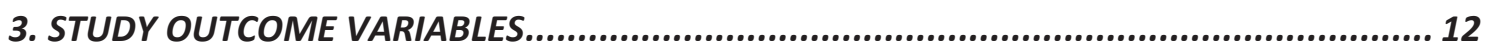

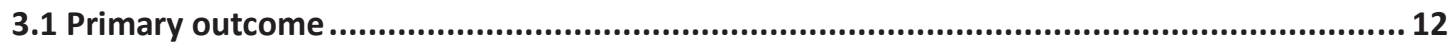

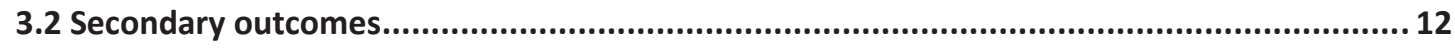

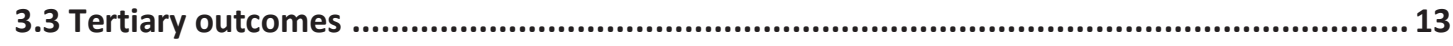

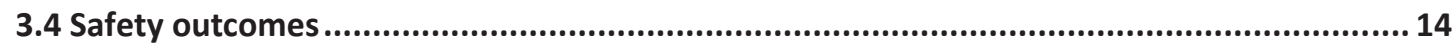

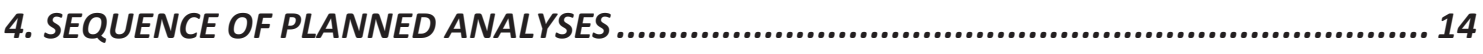

4.1 Interim analyses

4.2 Blinded reviews of primary outcome ...................................................................................... 14

4.3 Planned intervention group comparisons ....................................................................... 14

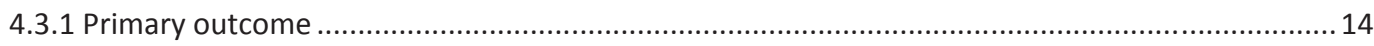

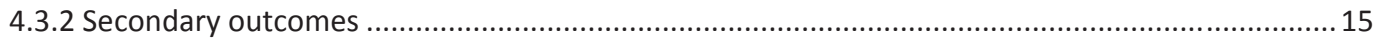

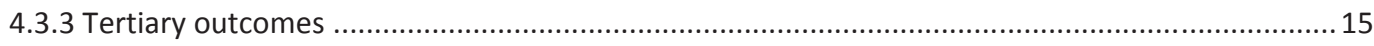

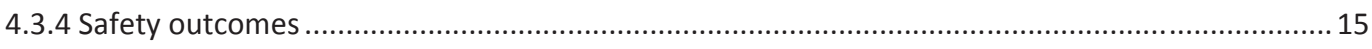

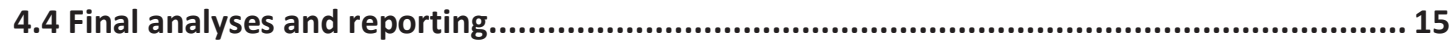

5. CHANGES TO STATISTICAL INFORMATION IN THE PROTOCOL .............................. 16 


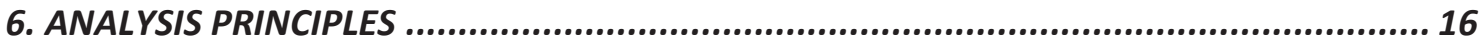

6.1 Intention-to-treat principle and analysis dataset .................................................................. 16

6.2 Multicentre study and heterogeneity ............................................................................. 17

6.3 Multiple comparisons and multiplicity................................................................................ 17

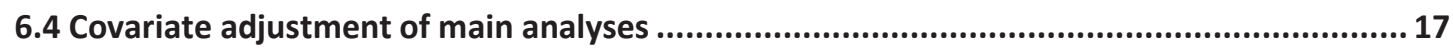

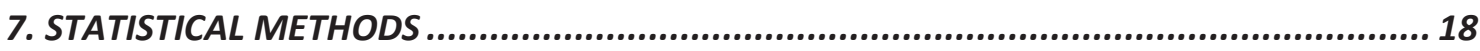

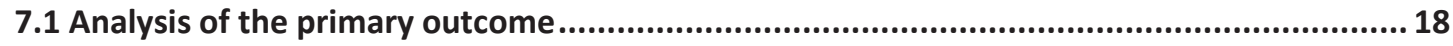

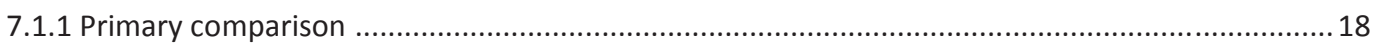

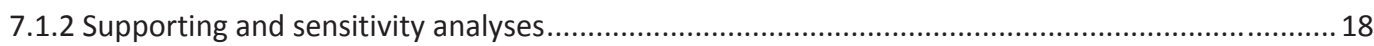

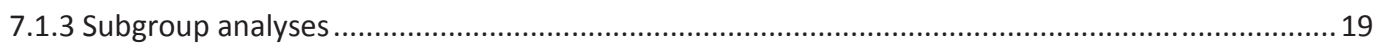

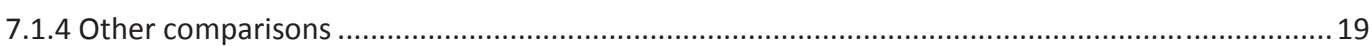

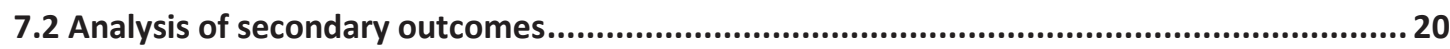

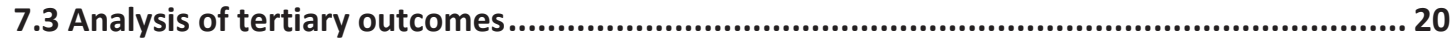

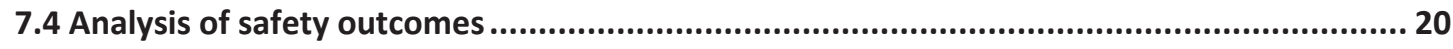

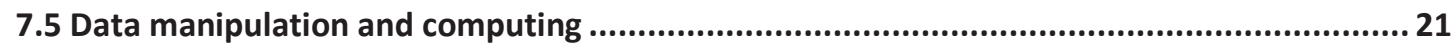

8. REPORTING

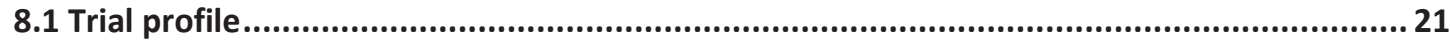

8.2 Patient characteristics and baseline comparisons ................................................. 21

8.3 TABLES, LISTINGS, AND FIGURES (TLFs) ......................................................... 22

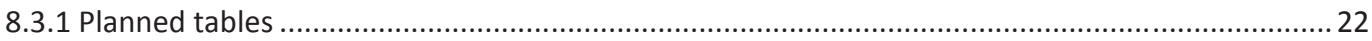

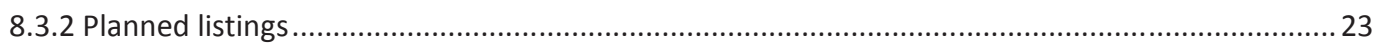

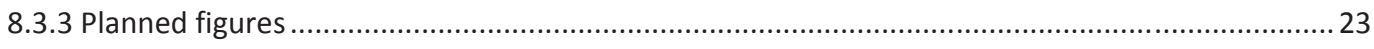

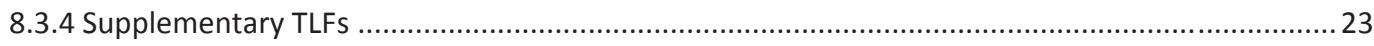

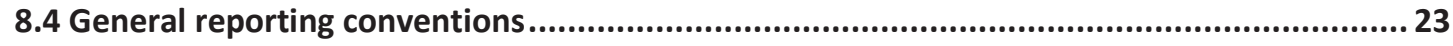

8.5 Statistical summary conventions .......................................................................... 24

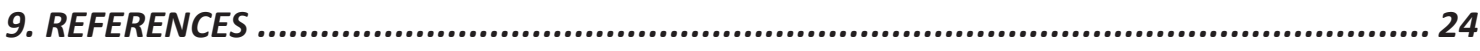




\section{INTRODUCTION}

\subsection{Background}

The incidence and prevalence of end-stage kidney disease (ESKD) are increasing due to aging of the population and growing rates of co-morbid conditions such as diabetes mellitus. Haemodialysis (HD) is the most common renal replacement therapy worldwide and a functioning vascular access is essential for optimal patient outcomes. Vascular access dysfunction is associated with significant morbidity and mortality and presents a major economic burden for health care providers (10-12). A native arteriovenous fistula (AVF) is the preferred type of vascular access due to lower rates of thrombosis, infection, interventions to maintain patency and overall mortality when compared with synthetic arteriovenous grafts (AVG) or central venous catheters (CVC) (13-15). However, native AVF take longer to establish and have a higher risk of failure to mature.

Primary failure rates of AVF have been reported to range from $20 \%$ to $60 \%$ with failure usually occurring as a result of early thrombosis or failure of the fistula to mature $(16,17)$. Strategies to improve early access survival by reducing thrombosis include short-term antiplatelet therapy and pre-operative identification by ultrasound of unsuitable anatomy. Although several small trials have assessed the effect of short-term post-operative use of antiplatelet agents such as aspirin, sulphinpyrazone, and ticlopidine (18-22) on early AVF thrombosis, these trials had many limitations including inadequate power, variable drug dosing and variable timing of drug administration. To date, there has been very little work focused on the important area of interventions to promote AVF maturation. Omega-3 polyunsaturated fatty acids (omega-3 PUFAs) found in fish oils exert biological effects that may improve both AVF maturation and patency due to pleiotropic biological actions, including inhibition of platelet aggregation, vasodilatation, and anti-inflammatory and antiproliferative effects by reducing the availability of arachidonic acid, leukotriene and cytokine production as well as increasing prostaglandin-13 production (23). Whilst fish oil reduces thrombosis rates in synthetic grafts when compared to placebo (24), there has been no trial to date examining the effect of fish oils on AVF outcomes.

\subsection{Study synopsis}

The FAVOURED trial is an international multi-centre, double-blind, randomised controlled trial designed to determine whether the use of omega-3 PUFAs when compared with matched placebo would reduce the AVF access failure rate in the 12 months following AVF surgery. The trial has more arms than needed to test the primary question because of midstream revisions of inclusion criteria to allow randomisation of patients taking aspirin as well as changes to the primary objective and outcome to focus on the effect of omega-3 PUFAs on a composite measure of AVF access failure (1).

Figure 1 displays the study schema. Eligible patients were classified according to their current aspirin-taking status. Patients who were taking aspirin and unable to cease (open-label aspirin) were randomly assigned to receive either omega-3 PUFAs or placebo capsules. Patients in the omega-3 PUFAs group received $4 \mathrm{gm}$ daily in the form of four Omacor capsules (46\% eicosapentaenoic acid (EPA) and 38\% docosapentaenoic acid (DHA), as the ethyl esters). Patients in the placebo group received 4 matching placebo capsules (olive oil). Both active and placebo capsules were supplied by BGP Products Operations GmBH. 
Patients who were either not taking aspirin or were taking aspirin but could stop were randomly assigned to one of four groups: omega-3 PUFAs plus low-dose oral aspirin (100 mg per day); omega-3 PUFAs plus matching placebo aspirin; placebo omega-3 PUFAs plus low-dose oral aspirin; or placebo omega-3 PUFAs plus placebo aspirin. Active and placebo aspirin tablets were supplied by Bayer Healthcare. For all groups, treatment commenced on the day prior to surgery and continued for 12 weeks.

To address the primary research question, two groups were formed by combining the three groups given omega-3 PUFAs (arms A, B, E) and the three groups given placebo omega-3 PUFAs (arms C, D, F). Aspirin use (no aspirin, randomised to aspirin, open-label aspirin) was expected to be balanced across the two combined omega-3 PUFAs groups (active and placebo) due to the separate randomisation schemes, one allocating approximately equal numbers of existing aspirin users to the two omega-3 PUFAs groups and the other allocating approximately equal numbers of non-aspirin users to the four groups in the original factorial design.

The planned sample size was 906 patients (includes adjustment for anticipated rates of treatment drop-out and non-compliance). Allowing for a 5\% loss to follow-up, the recruitment target was 954 patients. The trial began recruiting participants in August 2008 and stopped recruiting on 28 February 2014, before reaching the recruitment target. A total of 568 participants from 35 clinical centres in Australia, New Zealand, Malaysia and the United Kingdom were randomised, which corresponds to approximately $60 \%$ of the planned recruitment target. The central database is expected to be ready for analysis by July 2015 .

\subsection{Sub-studies}

There are three sub-studies associated with the FAVOURED trial: the red blood cell (RBC) membrane fatty acid sub-study; the dietary and physical questionnaire sub-study; and the presurgical doppler sub-study. The RBC fatty acid sub-study will assess compliance in use of omega-3 fatty acids and the association between RBCs and clinical and dietary baseline characteristics. The dietary and physical questionnaire sub-study will examine the relationship between dietary intake (especially fish) and lifestyle factors and their association with patient outcomes. The pre-surgical Doppler sub-study will examine relationships between pre-operative vascular characteristics determined by duplex ultrasound and AVF access outcomes. Analysis plans for these sub-studies will be documented separately. A fourth sub-study is described in the trial protocol, the platelet and coagulation function substudy, but it was abandoned due to difficulties in data collection.

\section{STUDY DESIGN ISSUES}

\subsection{Overview}

The study is a prospective, multi-centre, double-blind, randomised, placebo-controlled phasethree trial. 


\subsection{Study population}

The population of interest is chronic kidney disease (CKD) patients attending renal units (outpatients and dialysis) throughout Australia, New Zealand, Malaysia and the United Kingdom who have a planned AVF surgery.

\subsubsection{Inclusion criteria}

Patients were eligible for inclusion in the study if all the following criteria were met:

1. Stage 4 or 5 CKD

2. Currently on haemodialysis or haemodialysis is planned to start within 12 months (including patients currently on peritoneal dialysis)

3. Planned AVF will be the primary haemodialysis access mechanism

4. Surgery to create an AVF in the upper or lower arm is planned

5. Aged over 19 years

6. Treating team agreeable to patient's involvement in the trial

7. Able to give informed consent

\subsubsection{Exclusion criteria}

Patients were excluded from the study if any one of the following conditions was met:

1. Revision of existing AVF rather than de novo AVF

2. Medical indication for anticoagulants or anti-platelet agents*

3. Known intolerance of agents including hypersensitivity to aspirin, allergy to any other nonsteroidal anti-inflammatory drug (NSAID) or fish

4. Current use of aspirin within two weeks of commencing the trial, or of omega-3 PUFAs within 4 weeks of commencing the trial*

5. Pregnancy, lactation or intention to fall pregnant during the time course of the study

6. Known bleeding disorder or established diagnosis of active or suspected bleeding

7. History of gastrointestinal ulcers or bleeding within the last 3 months

8. Platelet count less than $100 \times 10^{9} / \mathrm{L}$

9. Known active peptic ulcer disease

10. Severe hepatic insufficiency

11. Already receiving anti-coagulation therapy such as warfarin

12. Receiving regular NSAID treatment for another indication such as arthritis

13. Syndrome of asthma, rhinitis and nasal polyps if uncontrolled on usual therapy

14. Plan to have other (non-access) surgery within 2 weeks of trial medication period where, in the opinion of the investigator, aspirin or omega-3 PUFAs would be contraindicated for the planned procedure

15. Potential non-compliance with treatment regimen in the view of the treating clinicians

16. Involved in another clinical trial where the intervention being trialled is likely to confound the outcome of this trial

17. Previously randomised to this trial

*These criteria were exclusions under the original protocol. Under the amended protocol, patients who met exclusion criterion 2 (Medical indication for anticoagulants or antiplatelet agents) due to aspirin use and/or exclusion criterion 4 (Current use of aspirin within two weeks of commencing trial) but were otherwise suitable were randomised to one of the open-label aspirin arms. 


\subsection{Study design and treatment allocation}

Before randomisation, participants were classified according to their aspirin-taking status: (1) not taking aspirin or taking aspirin but able to cease; (2) taking aspirin and unable to cease. Those in the first category were randomised 1:1:1:1 to one of four arms in a factorial design: active aspirin and active omega-3 PUFAs; active aspirin and placebo omega-3 PUFAs; placebo aspirin and active omega-3 PUFAs; placebo aspirin and placebo omega-3 PUFAs. Participants in the second category were randomised 1:1 to one of two parallel groups: active omega-3 PUFAs or placebo omega-3 PUFAs. Within both strata, interventions were allocated using adaptive minimisation with planned fistula site (lower vs upper arm) and study site (hospital or dialysis unit) as minimisation variables.

The overall design is illustrated in Figure 1. The key comparison is that between active omega-3 PUFAs (arms A, B and E) and placebo omega-3 PUFAs (arms C, D and F).

\section{Figure 1. Study schema}

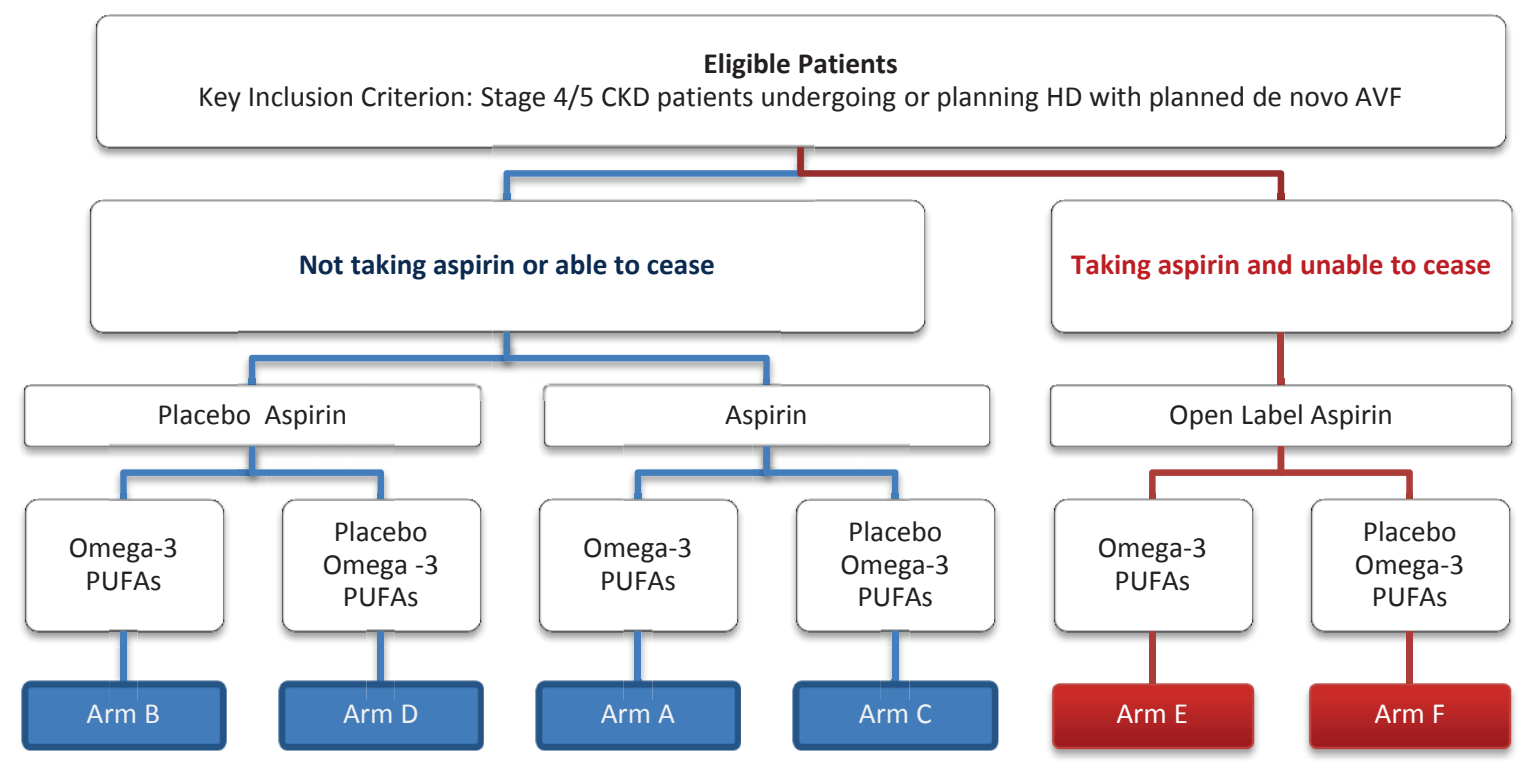

\subsection{Sample size}

The amended study was sized to detect a clinically relevant difference between omega-3 PUFAs and omega-3 PUFAs control groups (arms A, B and E combined versus C, D and F combined) on the primary composite outcome of AVF access failure. The 12- month postsurgery event rate for AVF access failure was assumed to be at least $30 \%$ in the control group. It was expected that the reduction in AVF access failure due to omega-3 PUFAs would be $30 \%$ (an absolute reduction in risk of $9 \%$ ). To detect a $30 \%$ relative risk reduction with $80 \%$ power and a significance level of 5\%, 734 participants would be required (367 per group). To allow for a 5\% drop-in from control to omega-3 PUFAs and a 5\% drop-out from omega-3 PUFAs to placebo, the total number of participants increased to 906 (453 per group). Allowing for a 5\% loss to follow-up, a total of 954 participants needed to be 
recruited. The assumed 30\% event rate for AVF access failure is conservative when compared to the $60 \%$ event rate reported by the North American clopidogrel Trial (25) and other recently published studies $(23,26)$. However, the vascular access practices and AVF outcomes in the United States differ from those in Australasia. Moreover, the report to the FAVOURED Data and Safety Management Board in December 2011 showed a 38\% blinded pooled event rate among the first 184 participants, indicating that a vascular access failure rate of $30 \%$ is a reasonable assumption.

\subsection{Treatment blinding and allocation concealment}

Investigators and patients were blinded to treatment assignment. Microbiology staff in local laboratories who performed outcome assessments were blinded to the patient's assigned treatment. To ensure concealment of treatment allocation, randomisation was performed using a central web-based randomisation system called Flexetrials ${ }^{\mathrm{TM}}$ administered by the National Health and Medical Research Council Clinical Trials Centre in Sydney.

\subsection{Schedule of assessments}

\begin{tabular}{|c|c|c|c|c|c|c|c|c|c|}
\hline \multirow{2}{*}{$\begin{array}{l}\text { Study Phase } \\
\text { Assessment }\end{array}$} & \multirow{2}{*}{$\begin{array}{c}\text { Baseline } \\
\leq 4 \text { wks prior } \\
\text { to surgery }\end{array}$} & \multirow[t]{2}{*}{ Surgery } & \multicolumn{3}{|c|}{$\begin{array}{l}\text { Treatment } \\
\text { Period (wks) }\end{array}$} & \multicolumn{4}{|c|}{$\begin{array}{c}\text { Follow-up Period } \\
\text { (mths) }\end{array}$} \\
\hline & & & 1 & 6 & 12 & 6 & 12 & 24 & 36 \\
\hline Informed Consent & $\mathrm{X}$ & & & & & & & & \\
\hline Medical History & $\mathrm{X}$ & & & & & & & & \\
\hline Weight and blood pressure & $\mathrm{X}$ & $\mathrm{X}$ & $\mathrm{X}$ & $\mathrm{X}$ & $\mathrm{X}$ & $\mathrm{X}$ & $\mathrm{X}$ & $\mathrm{X}$ & $\mathrm{X}$ \\
\hline Demographic characteristics & $\mathrm{X}$ & & & & & & & & \\
\hline Physical examination & $\mathrm{X}$ & & & & & & & & \\
\hline Dialysis status & $\mathrm{X}$ & & $\mathrm{X}$ & $\mathrm{X}$ & $\mathrm{X}$ & $\mathrm{X}$ & $\mathrm{X}$ & $\mathrm{X}$ & $\mathrm{X}$ \\
\hline Description and status of AVF & & $\mathrm{X}$ & $\mathrm{X}$ & $\mathrm{X}$ & $\mathrm{X}$ & $\mathrm{X}$ & $\mathrm{X}$ & $\mathrm{X}$ & $\mathrm{X}$ \\
\hline AVF Access Failure components & & $\mathrm{X}$ & $\mathrm{X}$ & $\mathrm{X}$ & $\mathrm{X}$ & $\mathrm{X}$ & $\mathrm{X}$ & & \\
\hline Concomitant medications & $\mathrm{X}$ & $\mathrm{X}$ & $\mathrm{X}$ & $\mathrm{X}$ & $\mathrm{X}$ & $\mathrm{X}$ & & & \\
\hline Adverse events & & $\mathrm{X}$ & $\mathrm{X}$ & $\mathrm{X}$ & $\mathrm{X}$ & $\mathrm{X}$ & & & \\
\hline \multicolumn{10}{|l|}{ Fasting Pathology Tests } \\
\hline Haematology and coagulation & $\mathrm{X}$ & & & & $\mathrm{X}$ & & & & \\
\hline Biochemistry and lipid profile & $\mathrm{X}$ & & & & $\mathrm{X}$ & & & & \\
\hline Urinary protein/creatinine ratio & $\mathrm{X}$ & & & & $\mathrm{X}$ & & & & \\
\hline Optional: Homocysteine and $\mathrm{Lp}(\mathrm{a})$ & $\mathrm{X}$ & & & & $\mathrm{X}$ & & & & \\
\hline \multicolumn{10}{|c|}{ Stored Samples for ANZ Sites (for central laboratory analysis) } \\
\hline $\mathrm{RBC}$ fatty acid sample, hsCRP & $\mathrm{X}$ & & & $\mathrm{X}$ & $\mathrm{X}$ & & & & \\
\hline $\begin{array}{l}\text { Urinary thromboxane B2 and } \\
\text { prostacyclin metabolites }\end{array}$ & $\mathrm{X}$ & & & & $\mathrm{X}$ & & & & \\
\hline
\end{tabular}




\section{STUDY OUTCOME VARIABLES}

\subsection{Primary outcome}

The primary outcome is AVF access failure (no, yes) within 12 months after AVF creation. AVF access failure is a composite outcome comprising three clinical components:

1. Thrombosis of the AVF: This is defined as the absence of a thrill or bruit by clinical assessment and/or requirement for rescue interventions including medical thrombolysis or surgical thrombectomy to restore patency for thrombosis or occlusion for the study AVF between AVF surgery and the month 12 visit.

2. AVF Abandonment: This is defined as the permanent abandonment of study AVF between AVF surgery and the month 12 visit. Events that may indicate AVF abandonment include thrombosis of the study AVF, imaging showing that the study AVF is unusable or not amenable to any intervention for its improvement, insertion of another dialysis access (new AVF, AVG, CVC or peritoneal dialysis access) or ligation of the study AVF due to thrombosis.

3. Cannulation Failure: This is defined as the failure to successfully cannulate the study AVF with 2 needles (or with 1 needle if using a single needle dialysis method) during 8 or more out of 12 haemodialysis sessions during the Cannulation Assessment Period (CAP). CAP is defined as the first 12 consecutive maintenance HD sessions after the week 12 visit (end of study intervention). HD sessions that occur before week 12 are not part of the CAP. A study AVF that is abandoned before the 12 month visit will be considered to have failed the CAP. Participants who do not commence HD before the 12 month visit are not assessable for cannulation failure.

The three components are being independently adjudicated by two observers, each blinded to the medical history of participants and their treatment assignment, and categorised as $0=$ no or $1=$ yes. Disagreements are being documented and will be resolved by consensus. AVF access failure will be coded $1=$ yes for participants with at least one component event and $0=$ no for participants with no component events.

\subsection{Secondary outcomes}

Data are being collected on a range of secondary outcome variables related to AVF access. The key secondary outcomes are the components of the primary composite outcome. The full list of secondary outcomes is as follows:

1. Thrombosis of the study AVF between AVF surgery and the month 12 visit, where thrombosis is a dichotomous outcome (no, yes) as defined for the primary composite outcome.

2. AVF abandonment between AVF surgery and the month 12 visit, where abandonment is a dichotomous outcome (no, yes) as defined for the primary composite outcome.

3. Cannulation failure between AVF surgery and the month 12 visit, where cannulation failure is a dichotomous outcome (no, yes) as defined for the primary composite outcome.

4. Primary patency (no, yes) of the AVF during the first 12 months after AVF surgery. Primary patency is defined as the presence of an audible bruit over the site of the arterio-venous anastomosis and is recorded at the following time points: within 24 hours post-surgery, and at visits 1, 6, and 12 weeks and 6 and 12 months after AVF surgery. 
5. Failure (no, yes) of a patent AVF to cannulate during the cannulation assessment period.

6. Number and type of interventions the study AVF required between AVF surgery and the month 12 visit. Interventions include rescue procedures designed to restore patency of the AVF (medical thrombolysis or surgical thrombectomy) and non-rescue procedures (surgical or radiological revision or dilatation of the AVF from or proximal to the anastomosis to the ipsilateral central vein, dilation of central venous stenosis, ligation of tributaries, superficialisation of AVF or ligation of fistula or salvage by DRIL [distal reconstruction and interval ligation] due to distal ischaemia). Specific outcomes will be:

a. Any intervention (no, yes)

b. Total number of interventions

c. Any rescue intervention (no, yes)

d. Total number of rescue interventions

e. Any non-rescue intervention (no, yes)

f. Total number of non-rescue interventions

7. Time to first thrombosis/rescue intervention is defined as the time from AVF creation to the first occasion of a rescue intervention up to the month 12 visit.

8. Time to AVF abandonment is defined as the time from AVF surgery to permanent abandonment of study AVF up to the 12 month visit.

9. Time to the first component event of AVF thrombosis/rescue intervention or AVF abandonment.

10. Time to first successful cannulation is defined as the time from the AVF surgery to the first successful attempt at access cannulation up to the 12 month visit.

11. CVC requirement from the end of the 12 -week study intervention period to the 12 month visit where $\mathrm{CVC}$ requirement is defined as the use of a CVC on any occasion to provide vascular access for haemodialysis (binary: no, yes).

12. Any CVC requirement during the CAP (no, yes)

13. Any CVC requirement between the end of the CAP and the 12 month visit (no, yes)

14. Number of days a CVC was in situ between the week 12 and 12 month visit

\subsection{Tertiary outcomes}

The following have been designated as tertiary outcome variables:

1. Any CVC requirement up to the 12 month visit (no, yes)

2. Long term outcomes of AVF:

a. AVF abandonment by the 24 month visit (no, yes)

b. AVF abandonment by the 36 month visit (no, yes)

c. Time to AVF abandonment (administratively censored at 36 months)

3. Accuracy (unsure/incorrect, correct) of participant's best guess about whether they received active or placebo omega-3 PUFA treatment - measured at the end of the treatment period

4. Accuracy (unsure/incorrect, correct) of physician's best guess about whether their patient had received active or placebo omega-3 PUFA treatment - measured at the end of the treatment period 


\subsection{Safety outcomes}

The following safety outcome variables have been recorded:

1. Any SAE

a. Death due to any cause

b. Any life-threatening event

c. Any initial or prolonged inpatient hospitalisation

d. Any persistent or significant disability/incapacity

e. Any important medical event

f. Any congenital abnormality/birth defect

2. SAE body system (10 categories)

3. SAE relationship to study medication (none, unlikely, possible, probable)

4. Any adverse drug reaction (ADR)

a. Any bleeding event

b. Any gastrointestinal event

c. Other event

5. ADR severity (mild, moderate, severe, life-threatening)

6. ADR relationship to study medication (possible, probable)

\section{SEQUENCE OF PLANNED ANALYSES}

\subsection{Interim analyses}

An independent Data and Safety Monitoring Board (DSMB) comprising experts in clinical trials, biostatistics, and nephrology was in charge of reviewing un-blinded data on patient characteristics, treatment compliance, and safety and efficacy outcomes. Two formal interim efficacy analyses were planned after $1 / 3$ and $2 / 3$ of total patients had been recruited and followed for at least 12 months. The Haybittle-Peto rule was used as a stopping guideline for efficacy (27). Due to cessation of recruitment well before the target sample size, only the first efficacy interim analysis was performed. The DSMB met on 20 March 2013 to consider the results of this analysis and recommended continuation of the trial. SAEs, including bleeding events, have been monitored on a regular basis by the DSMB. Only DSMB members and statisticians compiling the closed-session reports for DSMB meetings had access to unblinded interim data and results before database lock.

\subsection{Blinded reviews of primary outcome}

Blinded reviews of the primary outcome data were performed to check that the assumptions underlying the original sample size calculations remained plausible. These reviews were conducted at irregular intervals by clinicians and a statistician blinded to treatment allocation.

\subsection{Planned intervention group comparisons}

\subsubsection{Primary outcome}

The following intervention group comparisons are planned for the primary outcome: 
1. Omega-3 PUFAs (Arms A, B \& E) versus placebo omega-3 PUFAs (Arms C, D \& F)

2. Omega-3 PUFAs versus placebo omega-3 PUFAs under open label aspirin (Arm E vs F)

3. Aspirin (Arms A \& C) versus placebo aspirin (Arms B \& D)

4. Combined aspirin and omega-3 PUFAs (Arm A) versus combined placebo aspirin and placebo omega-3 PUFAs (Arm D)

5. Subgroups formed by:

a. Planned AVF site (lower arm, upper arm)

b. Actual AVF site (lower arm, upper arm)

c. Diabetes (no, yes)

d. Gender (female, male)

e. Age (quartiles)

f. Any cardiovascular disease (no, yes [peripheral vascular disease, cerebrovascular disease, ischaemic heart disease])

g. Dialysis at baseline (no [pre-dialysis, transplant], yes [haemodialysis, peritoneal dialysis])

\subsubsection{Secondary outcomes}

The following intervention group comparisons are planned for fourteen secondary outcomes:

1. Omega-3 PUFAs (Arms A, B \& E) versus placebo omega-3 PUFAs (Arms C, D \& F)

2. Omega-3 PUFAs versus placebo omega-3 PUFAs under open label aspirin (Arm E vs F)

3. Aspirin (Arms A \& C) versus placebo aspirin (Arms B \& D)

\subsubsection{Tertiary outcomes}

A single comparison is planned for each tertiary outcome variable: omega-3 PUFAs (Arms A, B, \& E) versus placebo omega-3 PUFAs (Arms C, D, \& F).

\subsubsection{Safety outcomes}

The following intervention group comparisons are planned for all safety outcomes:

1. Omega-3 PUFAs (Arms A, B, \& E) versus placebo omega-3 PUFAs (Arms C, D, \& F)

2. Omega-3 PUFAs versus placebo omega-3 PUFAs under open label aspirin (Arm E vs F)

3. Aspirin (Arms A \& C) versus placebo aspirin (Arms B \& D)

In addition, $\mathrm{ADR}$ s that are bleeding events will be summarised in more detail by comparing Arms A versus B versus $C$ versus D and Arms A+E versus B versus $C+F$ versus D.

\subsection{Final analyses and reporting}

All final planned analyses identified in the protocol and in this SAP will be performed only after the last patient has completed the 12 month post-surgery follow-up assessment and the database has been cleaned and locked. A blinded data review meeting will be held by the 
Trial Management Committee (TMC) prior to database lock. The database will not be locked, randomisation un-blinded or analysis commenced until this SAP has been approved by the TMC. Key statistics and trial results from the final analyses will be presented to the TMC for discussion prior to completion of the FSR and subsequent manuscripts. Any post-hoc exploratory analyses performed to provide support for planned analyses but not identified in this SAP will be documented and reported in appendices to the FSR and clearly identified as unplanned analyses in the text of the FSR.

\section{CHANGES TO STATISTICAL INFORMATION IN THE PROTOCOL}

The following amendments have been made to statistical information given in the original publication of the study protocol (22):

1. The primary outcome was changed from unassisted patency of the AVF at three months after randomisation to a composite measure of AVF access failure up to 12 months after AVF surgery. This amendment was in response to external results reported by Dember et al. (25).

2. The original $2 * 2$ factorial design was expanded to 6 groups to increase the recruitment rate by allowing recruitment of patients who were taking aspirin and unable to cease. These aspirin-taking patients were randomised to receive active omega-3 PUFAs or placebo omega-3 PUFAs. As a result of this amendment, omega-3 PUFAs became the primary focus of the trial and aspirin was downgraded to a secondary objective.

3. Following from the first two changes, the sample size was recalculated to accommodate the new primary outcome.

4. Additional subgroups have been pre-specified. The original protocol reported one subgroup (AVF site). The TMC revised the number of subgroups at a meeting held in December 2014 specifically to discuss details of the SAP.

With the exception of the change to subgroups, the above changes to the study design and methods were incorporated into a revised study protocol in November 2010 (7) and subsequently published (1).

\section{ANALYSIS PRINCIPLES}

\subsection{Intention-to-treat principle and analysis dataset}

Tests of the effects of treatment on the primary composite outcome and the key secondary outcomes (i.e., the three components of the composite) will be conducted as close as possible to the intention-to-treat (ITT) ideal. All randomised patients who undergo their scheduled AVF surgery will be included in the "full analysis set" and analysed in the group to which they were randomly allocated regardless of whether the y received the assigned treatment and irrespective of any protocol deviations or violations. Analyses of these and other efficacy outcome variables will exclude the following randomised patients: (1) those who did not have an AVF created, either because their scheduled AVF surgery was cancelled, AVG surgery was performed instead, or an AVF was attempted but did not successfully form; (2) those who died within 12 months of surgery and were not assessed on any of the three components of the primary outcome before their death; and (3) those who withdrew from study treatment 
and withdrew consent for use of their data. These discontinuations will be captured in a figure illustrating participant progression through the trial.

Missing primary outcome data will be imputed using a method based on assumptions that are clinically defensible and unlikely to be biased in favour of the omega-3 PUFAs intervention. Robustness of results to the assumptions underlying the imputation method will be evaluated using a global sensitivity analysis approach which involves analyses based on different assumptions that trend towards best and worst case scenarios (28). Imputation and sensitivity analysis methods for the primary outcome are described in section 7.1.

\subsection{Multicentre study and heterogeneity}

Thirty-five dialysis centres recruited between one and 50 participants. Although included as a variable in the randomisation scheme, study centre will not be adjusted for in the main analyses of primary and key secondary efficacy outcomes because some centres were small ( $\leq 5$ participants) and may not contribute any events to the pooled data. However, if there is a positive treatment effect on the primary composite outcome and/or the component events, study centres will be combined within five Australian states, within Malaysia, and within a pooled New Zealand/United Kingdom (seven regional strata in total) to assess the homogeneity of the treatment effect across regions. Potential heterogeneity will be tested by including a treatment-by-region interaction term in a multivariable model with treatment and region as main effects.

\subsection{Multiple comparisons and multiplicity}

The Heybittle-Peto rule with a maximum of three analyses (two interims and one final) was used to control the overall Type 1 error rate for analysis of the effect of omega-3 PUFAs on the composite primary outcome variable. The trial continued after the first of two planned interim analyses. The second interim analysis was not performed due to early cessation of recruitment and so the final analysis will be assessed against an alpha of 0.05 . There will be no further adjustments for multiplicity as there is a pre-defined hierarchy of importance of outcome variables and study objectives and the influence of individual results on the overall interpretation of the trial will reflect their level within this hierarchy. Further, there are a limited number of subgroups, all of which are pre-specified. All statistical tests of significance will be two-sided.

\subsection{Covariate adjustment of main analyses}

The main statistical analyses of primary and secondary efficacy outcomes comparing active omega-3 PUFAs (Arms A, B \& E) with placebo omega-3 PUFAs (Arms C, D \& F) will be adjusted for aspirin strata (taking aspirin at randomisation, randomised to aspirin, randomised to placebo aspirin). Supporting analyses will be additionally adjusted for regional location of the clinical site and six pre-specified prognostic baseline characteristics (section 7.1.3). Comparisons of other groups on the primary and secondary efficacy outcomes will be unadjusted, with supporting analyses adjusted as described for the main omega-3 PUFA comparison. 


\section{STATISTICAL METHODS}

\subsection{Analysis of the primary outcome}

The primary outcome is AVF access failure within 12 months of AVF creation due to one or more of AVF thrombosis/rescue intervention, AVF abandonment, and AVF cannulation failure. Each component will be coded as no event $=0$ and an event $=1$. The composite will be coded as no component events $=0$ and at least one component event $=1$. There are two broad reasons why data may be missing on one or more AVF access failure components: the participant withdrew from the study within 12 months of AVF surgery by choice, due to receipt of a kidney transplant, or was lost to follow-up and was not assessed on all three components of the primary outcome before withdrawal; the participant completed 12 months of follow-up but did not commence HD and was not assessed for cannulation during the 12 month follow-up period. Participants with missing data on a component will be randomly allocated a zero or one according to the proportion of events in participants with valid data for the component. This method is consistent with an assumption that data are missing completely at random (MCAR) and justifiable on the grounds that the main reasons for withdrawal are unlikely to be related to the study treatment or the status of the patient's fistula.

\subsubsection{Primary comparison}

Log binomial regression will be used to compare omega-3 PUFAs (Arms A, B \& E) to placebo PUFAs (Arms C, D \& F) on AVF access failure. The analysis will be adjusted for aspirin-taking strata (randomised to active aspirin, randomised to placebo aspirin, open-label aspirin). In the log binomial regression model, the omega-3 PUFA versus placebo PUFA groups will be represented as a binary indicator variable and aspirin taking strata ( 3 groups) will be represented as two binary indicator variables with aspirin placebo as the reference group. The main result will be the treatment effect risk ratio and $95 \%$ confidence interval adjusted for aspirin strata.

\subsubsection{Supporting and sensitivity analyses}

Robustness of the estimate of the omega-3 PUFA treatment effect will be assessed by an analysis which additionally adjusts for baseline characteristics either known or a priori suspected to be associated with the primary outcome (29). The relevant baseline characteristics are: planned AVF site (lower arm, upper arm), diabetes (no, yes), gender (female, male), age (years), any cardiovascular disease (no, yes [peripheral vascular disease, cerebrovascular disease, ischaemic heart disease]), and dialysis at baseline (no [pre-dialysis, transplant], yes [haemodialysis, peritoneal dialysis]). This covariate adjusted model will include main effects for treatment group and the six covariates but will not include interaction terms. An adjusted analysis will be viewed as supportive, providing additional context for interpreting the primary unadjusted analysis. If there is a statistically significant effect of omega-3 PUFAs on AVF access failure, heterogeneity of the effect will be tested by including a treatment by region (Australian state, Malaysia, New Zealand/United Kingdom) interaction effect in the primary and robustness models.

Sensitivity of the result of the primary treatment comparison to single imputation of a trialspecific representative proportion of events for each component will be assessed as follows: 
1. Repeat the log binomial regression analysis on best- and worst-case single imputation scenarios. In the former, all missing values on component events in the omega-3 group will be assumed to be non-events and all those in the placebo omega-3 group will be assumed to be events. The worst-case scenario will be based on the reverse pair of assumptions. In addition, a range of intermediate scenarios will be assessed.

2. Repeat the log binomial regression analysis on complete cases. This analysis will be performed on the composite outcome but include data only from participants with observed data on all three components.

3. Perform a maximum likelihood-based analysis using observations on the three components. Participants with no observed data will not contribute to the maximum likelihood estimate of component and composite relative risks.

4. Analyse the composite outcome using a pattern mixture model allowing for two dominant patterns of missing component (and therefore composite) data: participants who completed 12 months of follow-up but had incomplete data because their AVF was not assessed for cannulation and they did not have an event on the other two components; and participants who withdrew before the 12 month visit.

These sensitivity analyses are variously designed to address potential bias in the main estimate of treatment effect and the main problem with single imputation, namely that the method does not take account of uncertainty pertaining to missing data with the result that the estimate of variability in the treatment effect may be biased downwards and inflate Type I error above the nominal 5\% level.

\subsubsection{Subgroup analyses}

Pre-specified subgroups are formed by the following baseline characteristics: planned AVF site (lower arm, upper arm), actual AVF site (lower arm, upper arm), diabetes (no, yes), gender (female, male), age (quartiles), any cardiovascular disease (no, yes [peripheral vascular disease, cerebrovascular disease, ischaemic heart disease]), and dialysis at baseline (no [pre-dialysis, transplant], yes [haemodialysis, peritoneal dialysis]). The main subgroup analyses will be tests of treatment-by-subgroup variable interactions in log binomial regression models adjusted for aspirin strata. These analyses will be performed regardless of the result of the log binomial model for the primary comparison. Risk ratio estimates of treatment effect (omega-3 PUFAs versus placebo PUFAs) within each sub-group will be reported along with their $95 \%$ confidence limits.

\subsubsection{Other comparisons}

Log binomial regression models will be used to compare omega-3 PUFAs and placebo PUFAs under open label aspirin (Arm E versus F), aspirin and placebo aspirin (Arms A \& C versus B \& D), and combined aspirin and omega-3 PUFAs (Arm A) relative to placebo aspirin and placebo PUFAs (Arm D). These models will have a single binary indicator variable representing the treatment group comparison and will not be adjusted for other characteristics. The second model assumes an additive effect for aspirin and omega-3 PUFAs which will be assessed by initially including a treatment (aspirin or placebo aspirin) by treatment (PUFAs or placebo PUFAs) interaction term in the log binomial model. Supporting and sensitivity analyses performed for the primary comparison will be performed for these secondary comparisons. 


\subsection{Analysis of secondary outcomes}

Secondary outcome variables are variously binary, count, and time-to-event data. Binary outcomes with a single measurement per participant such as the three components of the primary outcome will be analysed using a log binomial regression model to obtain estimates of risk ratios. Primary patency is a binary outcome which is assessed repeatedly within participants. To accommodate the within participant correlation, this outcome will be analysed using a generalised estimating equation approach for relative risks with an unstructured variance-covariance matrix (Toeplitz if convergence fails). Count outcomes will be analysed with Poisson regression models. If there is under- or over-dispersion, residuals will be examined to determine the most suitable model (e.g., quasi-Poisson regression, negative binomial regression). Time-to-event outcomes will be analysed with a competing risks regression model. Competing risks for time to first rescue intervention and time to first successful cannulation will be AVF abandonment, death and renal transplant. Competing risks for time to AVF abandonment will be death and renal transplant. As there will be relatively few competing risk events, these analyses will be performed with a composite competing risk group. Participants who did not experience the relevant outcome event or a competing risk event by 12 months after surgery will be censored at this time and those who withdrew from the study will be censored at the time of withdrawal.

Omega-3 PUFAs (Arms A, B \& E) will be compared to placebo PUFAs (Arms C, D \& F) on all secondary outcomes using a statistical model appropriate for the outcome. The same statistical models will be used to compare omega-3 PUFAs versus placebo PUFAs under open label aspirin (Arm E versus F) and aspirin (Arms A \& C) versus placebo aspirin (Arms $\mathrm{B} \& \mathrm{D})$ for participants who were not taking aspirin or able to cease before randomisation.

\subsection{Analysis of tertiary outcomes}

Tertiary outcome variables are binary and time-to-event data. Omega-3 PUFAs (Arms A, B \& E) will be compared to placebo PUFAs (Arms C, D \& F) on all tertiary outcomes using a statistical model appropriate for the type of outcome as described for secondary outcomes.

\subsection{Analysis of safety outcomes}

All treatment groups will be compared on safety outcomes: omega-3 PUFAs (Arms A, B \& E) versus placebo PUFAs (Arms C, D \& F); omega-3 PUFAs versus placebo PUFAs under open label aspirin (Arm E versus F); and aspirin (Arms A \& C) versus placebo aspirin (Arms B \& D) for participants who were not taking aspirin or able to cease before randomisation. The relationship of each category of SAE and ADR (present versus absent) to treatment group will be summarised by frequencies and percentages and analysed with chi-square tests. The relationship between treatment group and SAE body system (10 categories), SAE relationship to study medication (none, unlikely, possible, probable), type of ADR event (bleeding, gastrointestinal, other), ADR severity (mild, moderate, severe, life-threatening), and ADR relationship to study medication (possible, probable) will be summarised by frequencies and percentages and analysed with chi-square tests. The same methods will be used to summarise in more detail ADRs that are bleeding events. The more detailed summaries will compare Arms A versus B versus $C$ versus D and Arms A+E versus B versus $\mathrm{C}+\mathrm{F}$ versus $\mathrm{D}$. 


\subsection{Data manipulation and computing}

All trial data collected after randomisation are stored in a central InForm ${ }^{\circledR}$ database. Most data manipulation, tables, figures, listings and analyses will be documented in $\mathrm{SAS}^{\circledR}$ programs and performed using SAS version 9.4. Other data preparation and analyses will be documented in Stata $^{\circledR}$ programs and performed using Stata version 13.1.

\section{REPORTING}

All results described above as well as tables, listings and figures (TLFs) listed below will be presented in the FSR.

\subsection{Trial profile}

All patients who provide informed consent will be accounted for in the FSR. A CONSORTstyle flow diagram will illustrate patient progression through the trial from initial screening for eligibility to completion of the final primary outcome assessment. Number (percentage) of participants randomised to each treatment group will be given for all randomised patients along with reasons for study withdrawal by treatment group.

\subsection{Patient characteristics and baseline comparisons}

Demographic and other baseline characteristics will be summarised by assigned treatment group. Categorical variables will be summarised by frequencies and percentages. Percentages will be calculated according to the number of patients for whom data are available. Where values are missing, the denominator, which will be less than the number of patients assigned to the treatment group, will be reported either in the body or a footnote in the summary table. Continuous variables will be summarised by mean and standard deviation as well as quartiles.

Baseline demographic and clinical characteristics and laboratory investigations will be presented in three tables (see Section 6.3 for further details). Each table will include the following baseline demographic and clinical characteristics: gender, age at randomisation, ethnicity (Aboriginal/Torres Strait Islander [ATSI], Asian, Caucasoid, Maori/Pacific Islander [MPI], Other, Unknown), planned AVF location (upper arm, lower arm), height, weight, Body Mass Index (BMI), waist measurement $(\mathrm{cm})$, hip measurement $(\mathrm{cm})$, waist/hip ratio, smoking status (never, former, current), systolic blood pressure ( $\mathrm{mm} \mathrm{Hg}$ ), diastolic blood pressure ( $\mathrm{mm} \mathrm{Hg}$ ), heart rate, diabetes (no, yes), type of diabetes $(1,2)$, primary cause of endstage renal failure (diabetic neuropathy, glomerulonephritis, hypertension/vascular, polycystic kidney disease, reflux nephropathy, other, unknown), ischaemic heart disease (no, yes), congestive heart disease (no, yes), hypertension (no, yes), cerebrovascular disease (no, yes), peripheral vascular disease (no, yes), currently on renal replacement therapy (no, yes), type of current renal replacement therapy (transplant, automated peritoneal dialysis, continuous ambulatory peritoneal dialysis, haemodialysis), principal access currently in use for dialysis (AVF, AVG, CVC, PD catheter).

Each table will include the following baseline blood and biochemistry investigations: haemoglobin $(\mathrm{g} / \mathrm{L})$, platelets $\left(10^{\wedge} 9 / \mathrm{L}\right)$, white blood cells $\left(10^{\wedge} 9 / \mathrm{L}\right)$, International Normalised Ratio ([INR]), Activated Partial Thromboplastin Time ([APTT] sec), fibrinogen (mg/dL), 
sodium $(\mathrm{mmol} / \mathrm{L})$, potassium $(\mathrm{mmol} / \mathrm{L})$, bicarbonate $(\mathrm{mmol} / \mathrm{L})$, urea $(\mathrm{mmol} / \mathrm{L})$, creatinine $(\mu \mathrm{mol} / \mathrm{L})$, albumin $(\mathrm{g} / \mathrm{dL})$, calcium $(\mathrm{mmol} / \mathrm{L})$, phosphate $(\mathrm{mmol} / \mathrm{L})$, fasting glucose $(\mathrm{mmol} / \mathrm{L}$ [separately for diabetics and non-diabetics]), HbA1c ( $\%$ [separately for diabetics and nondiabetics]), parathormone ( $\mathrm{pmol} / \mathrm{L})$, triglyceride $(\mathrm{mmol} / \mathrm{L})$, cholesterol $(\mathrm{mmol} / \mathrm{L}), \mathrm{HDL}$ cholesterol ( $\mathrm{mmol} / \mathrm{L}), \mathrm{LDL}$ cholesterol $(\mathrm{mmol} / \mathrm{L})$, and urine protein:creatinine ratio $(\mathrm{mg} / \mathrm{mmol})$.

\subsection{TABLES, LISTINGS, AND FIGURES (TLFS)}

Templates for all planned TLFs are in a separate document titled FAVOURED SAP Tables, Listings and Figures. Where a TLF gives results by treatment group, unless otherwise stated, there should be three versions of the TLF:

a) All randomised participants: active omega-3 PUFAs (arms A, B, E) compared with placebo omega-3 PUFAs (arms C, D, F)

b) Participants randomised within the open-label aspirin stratum: active omega-3 PUFAs (arm E) compared with placebo omega-3 PUFAs (arm F)

c) Participants randomised within the non-aspirin-taking stratum: active aspirin (arms A and C) compared with placebo aspirin (arms B and D).

\subsubsection{Planned tables}

The following are planned summary tables:

Table 1. Enrolment by study centre

Table 2. Treatment group by study centre (parts a to c)

Table 3. Aspirin stratification at randomisation (open-label versus randomised) by study centre

Table 4. Protocol deviations and violations by treatment group (parts a to c)

Table 5. Protocol deviations and violations by aspirin stratification at randomisation (openlabel versus randomised)

Table 6. Medication compliance and withdrawals by treatment group (parts a to c)

Table 7. Medication compliance and withdrawals by aspirin stratification at randomisation (open-label versus randomised)

Table 8. Demographic and clinical baseline characteristics by treatment group (parts a to c)

Table 9. Demographic and clinical baseline characteristics by aspirin stratification at randomisation (open-label versus randomised)

Table 10. Baseline blood investigations by treatment group (parts a to c)

Table 11. Baseline blood investigations by aspirin stratification at randomisation (open-label versus randomised)

Table 12. Clinical assessments by treatment group across study visits (parts a to c)

Table 13. Primary outcome by treatment group (parts a to c)

Table 14. Primary outcome for subgroups by omega-3 PUFA treatment group (arms A, B, \& E versus $C, D, \& F$ )

Table 15. Secondary outcomes by treatment group (parts a to c): components of the primary composite outcome

Table 16. Secondary outcomes by treatment group (parts a to c): primary patency

Table 17. Secondary outcomes by treatment group (parts a to c): number and type of AVF interventions

Table 18. Secondary outcomes by treatment group (parts a to c): CVC requirement 
Table 19. Long term outcomes of AVF by omega-3 PUFA group (active vs placebo)

Table 20. Participant and physician best guess about participant's omega-3 PUFA group by participant's actual omega-3 PUFA group

Table 21. Any ADR and ADR categories by treatment group (parts a to c)

Table 22. ADR severity by treatment group (parts a to c)

Table 23. ADR relationship to study medication by treatment group (parts a to c)

Table 24. Any SAE and SAE categories by treatment group (parts a to c)

Table 25. Relationship of SAE to study medication by treatment group (parts a to c)

Table 26. SAE body system by treatment group (parts a to c)

\subsubsection{Planned listings}

The following are planned data and patient listings:

Listing 1. Reasons randomised participants did not have surgery

Listing 2. Reasons randomised participants had AVG rather than AVF surgery

Listing 3. Reasons for participants withdrawing from the study

Listing 4. Medication compliance problems requiring participant to come off trial

Listing 5. Deaths and life threatening events

\subsubsection{Planned figures}

The following are planned summary figures:

Figure 1. Monthly and cumulative entry of participants into the study

Figure 2. Flowchart of patient progression through the study

Figure 3. Forest plot of odds ratios and $95 \%$ confidence intervals for subgroup analyses (primary composite outcome and components of the composite)

Figure 4. Cumulative incidence functions for time to first thrombosis/rescue intervention by treatment groups (parts a to c)

Figure 5. Cumulative incidence functions for time to AVF abandonment by treatment groups (parts a to c)

Figure 6. Cumulative incidence functions for time to first thrombosis/rescue intervention or AVF abandonment by treatment groups (parts a to c)

Figure 7. Cumulative incidence functions for time to first successful cannulation by treatment groups (parts a to c)

\subsubsection{Supplementary TLFs}

Results from supporting and sensitivity analyses will be presented in supplementary tables. Missing data on the primary outcome will be summarised by treatment groups and presented in supplementary tables and figures as appropriate.

\subsection{General reporting conventions}

All TLFs will be presented in portrait orientation, unless landscape orientation suggests that the information is easier to view. Legends will be used for all figures with more than one variable or item displayed. Figure lines should be wide enough to see the line after being copied. 
All titles will be centred on a page. The first title line will be the number of the table, figure, or data listing. The second (and if required, third) line will be the description of the table, figure, or data listing. The ICH numbering convention will be used for all TLFs (30).

All tables, figures, and data listings will have the name of the relevant SAS (or Stata) program and a date-time stamp on the bottom of each output. All analysis programs developed for a table, figure, or data listing will be self-contained to facilitate transfer of programs to multiple computing environments. A separate analysis program will be written to produce each table.

\subsection{Statistical summary conventions}

For tables, sample sizes for each treatment group will be presented as totals in the column header $(\mathrm{N}=\mathrm{xxx})$, where appropriate. Sample sizes shown with summary statistics are the number (n) of patients with non-missing values.

Summaries for categorical variables will include only categories that patients had a response in. Percentages corresponding to null categories (cells) will be suppressed. All summaries for continuous variables will include: $\mathrm{N}$, mean, and SD. Other summaries (e.g. median, quartiles, $5 \%, 95 \%$ intervals, coefficient of variation $(\mathrm{CV})$ or $\% \mathrm{CV}$ will be used as appropriate. All percentages should be rounded and reported to a single decimal place (xx.X\%). If percentages are reported as integers, percentages greater than $0 \%$ but $<1 \%$ will be reported as $<1 \%$, whereas percentages greater than $99 \%$ but $<100 \%$ will be reported as $>99 \%$. A percentage of $100 \%$ will be reported as $100 \%$. No value of $0 \%$ should be reported. Any computation of percent that results in $0 \%$ is to be reported as a blank. Summaries that include p-values will report the $\mathrm{p}$-value to three decimal places with a leading zero (0.001). P-values $<0.001$ will be reported as $<0.001$.

\section{REFERENCES}

1. Viecelli AK, Pascoe E, Polkinghorne KR, Hawley C, Paul-Brent PA, Badve SV, et al. The Omega-3 fatty acids (Fish Oils) and Aspirin in Vascular access OUtcomes in Renal Disease (FAVOURED) study: the updated final trial protocol and rationale of post-initiation trial protocol modifications. BMC Nephrology. 2015(16).

2. ICH. ICH harmonised tripartite guideline: statistical principles for clinical trials E9. 1998.

3. ASA. Ethical guidelines for statistical practice. Prepared by the Committee on Professional Ethics.1999. Available from: http://www.amstat.org/about/ethicalguidelines.cfm.

4. NZSA. Code of Conduct of the New Zealand Statistical Association. 1995. Available from: http://stats.org.nz/code of conduct.shtml.

5. RSS. The Royal Statistical Society: Code of Conduct.1993. Available from: http://www.rss.org.uk/uploadedfiles/documentlibrary/142.pdf.

6. SSAI. The Statistical Society of Australia Inc. Code of Conduct.

7. The FAVOURED Trial Protocol, Version 9, 30 November 2009.

8. The FAVOURED Trial Charter of the Data and Safety Monitoring Board (DSMB), 13 June 2011.

9. ICH. ICH Harmonised tripartite guideline: guideline for good clinical practice E6. 1996.

10. Feldman HI, Held PJ, Hutchinson JT, Stoiber E, Hartigan MF, Berlin JA. Hemodialysis vascular access morbidity in the United States. Kidney International. 1993;43(5):1091-6. 
11. Feldman HI, Kobrin S, Wasserstein A. Hemodialysis vascular access morbidity. Journal of the American Society of Nephrology. 1996;7(4):523-35.

12. Manns B, Tonelli M, Yilmaz S, Lee H, Laupland K, Klarenbach S, et al. Establishment and maintenance of vascular access in incident hemodialysis patients: a prospective cost analysis. Journal of the American Society of Nephrology. 2005;16(1):201-9.

13. Allon M, Robbin ML. Increasing arteriovenous fistulas in hemodialysis patients: problems and solutions. Kidney International. 2002;62(4):1109-24.

14. Pastan S, Soucie JM, McClellan WM. Vascular access and increased risk of death among hemodialysis patients. Kidney International. 2002;62(2):620-6.

15. Polkinghorne KR, McDonald SP, Atkins RC, Kerr PG. Vascular access and all-cause mortality: a propensity score analysis. Journal of the American Society of Nephrology. 2004;15(2):477-86.

16. Andrassy K, Malluche H, Bornefeld H, Comberg M, Ritz E, Jesdinsky H, et al. Prevention of p.o. clotting of av. cimino fistulae with acetylsalicyl acid. Results of a prospective double blind study. Klinische Wochenschrift. 1974;52(7):348-9.

17. Kaufman JS. Antithrombotic agents and the prevention of access thrombosis. Seminars in Dialysis. 2000;13(1):40-6.

18. Engstrom K, Wallin R, T. S. Effect of low-dose aspirin in combination with stable fish oil on whole blood production of eicosanoids. Prostaglandins, leukotrienes, and essential fatty acids. 2001;64(6):291-7.

19. Fiskerstrand CE, Thompson IW, Burnet ME, Williams P, Anderton JL. Double-blind randomized trial of the effect of ticlopidine in arteriovenous fistulas for hemodialysis. Artificial organs. 1985;9(1):61-3.

20. Grontoft KC, Larsson R, Mulec H, Weiss LG, Dickinson JP. Effects of ticlopidine in AVfistula surgery in uremia. Fistula Study Group. Scandinavian journal of urology and nephrology. 1998;32(4):276-83.

21. Grontoft KC, Mulec H, Gutierrez A, Olander R. Thromboprophylactic effect of ticlopidine in arteriovenous fistulas for haemodialysis. Scandinavian Journal of urology and nephrology 1985;19(1):55-7.

22. Irish A, Dogra G, Mori T, Beller E, Heritier S, Hawley C, et al. Preventing AVF thrombosis: the rationale and design of the Omega-3 fatty acids (Fish Oils) and Aspirin in Vascular access OUtcomes in REnal Disease (FAVOURED) study. BMC Nephrology. 2009;10:1.

23. Schinstock CA, Albright RC, Williams AW, Dillon JJ, Bergstralh EJ, Jenson BM, et al. Outcomes of arteriovenous fistula creation after the Fistula First Initiative. Clinical Journal of the American Society of Nephrology. 2011;6(8):1996-2002.

24. Lok CE, Moist L, Hemmelgarn BR, Tonelli M, Vazquez MA, Dorval M, et al. Effect of fish oil supplementation on graft patency and cardiovascular events among patients with new synthetic arteriovenous hemodialysis grafts: a randomized controlled trial. JAMA. 2012;307(17):1809-16.

25. Dember LM, Beck GJ, Allon M, Delmez JA, Dixon BS, Greenberg A, et al. Effect of clopidogrel on early failure of arteriovenous fistulas for hemodialysis: a randomized controlled trial. Journal of the American Medical Association. 2008;299(18):2164-71.

26. Biuckians A, Scott EC, Meier GH, Panneton JM, Glickman MH. The natural history of autologous fistulas as first-time dialysis access in the KDOQI era. Journal of vascular surgery. 2008;47(2):415-21; Discussion 20-1.

27. Jennison C, Turnbull BW. Group sequential methods with applications to clinical trials. Washington, D.C.: Chapman \& GHall/CRC; 2000.

28. (U.S.). NRC. The prevention and treatment of missing data in clinical trials. Washington, DC.: National Academies Press.; 2010. 
29. Pocock SJ, Assmann SE, Enos LE, Kasten LE. Subgroup analysis, covariate adjustment and baseline comparisons in clinical trial reporting: current practice and problems. Statist Med. 2002;21:2917-30.

30. ICH. ICH Harmonised Tripartite Guideline: Structure and Content of Clinical Study Reports E3. 1995. 


\section{Supplementary Online Content}

Irish AB, Viecelli AK, Hawley CM, et al; for the Omega-3 Fatty Acids (Fish Oils) and Aspirin in Vascular Access Outcomes in Renal Disease (FAVOURED) Study Collaborative Group. Effect of fish oil supplementation and aspirin use on arteriovenous fistula failure in patients requiring hemodialysis: a randomized clinical trial. JAMA Intern Med. Published online January 3, 2017. doi:10.1001/jamainternmed.2016.8029

eTable 1. Cannulation Assessment Periods eTable 2. Primary and Secondary Outcomes for Treatment Combinations of Participants Randomized to the Factorial 2x2 Factorial Design $(n=388)$ eTable 3. Summary of Missing Data and Single Imputation across Components and the Composite for $n=536$ participants eTable 4. First Cannulation by Treatment Groups eTable 5. Months to Hemodialysis Initiation for Pre-dialysis Participants by Treatment Groups

eTable 6. Timing of First Renal Replacement Therapy for Pre-dialysis Participants by Treatment Groups

eFigure 1. Study Schema eFigure 2. Forest Plot for Sensitivity Analysis Fish oil versus Placebo and Aspirin versus Placebo eFigure 3. Erythrocyte Fatty Acid Composition by Study Visit and Treatment: Eicosapentaenoic Acid, Docosahexaenoic Acid, and EPA and DHA Combined

This supplementary material has been provided by the authors to give readers additional information about their work.

(c) 2017 American Medical Association. All rights reserved. 
eTable 1. Cannulation Assessment Periods

\begin{tabular}{|c|c|c|}
\hline $\begin{array}{l}\text { Start of maintenance } \\
\text { hemodialysis }\end{array}$ & Beginning of CAP & Duration of CAP \\
\hline Prior to week 12 visit & $\begin{array}{c}\text { First hemodialysis session } \\
\text { after week } 12 \text { visit }\end{array}$ & $\begin{array}{c}\text { First } 12 \text { consecutive } \\
\text { hemodialysis sessions }\end{array}$ \\
\hline $\begin{array}{c}\text { Between week } 12 \text { and month } \\
12 \text { visit }\end{array}$ & First hemodialysis session & $\begin{array}{c}\text { First } 12 \text { consecutive } \\
\text { hemodialvsis sessions }\end{array}$ \\
\hline After month 12 visit & No CAP & No CAP \\
\hline
\end{tabular}

(C) 2017 American Medical Association. All rights reserved. 
eTable 2: Primary and Secondary Outcomes for Treatment Combinations of Participants Randomized to the Factorial 2x2 Factorial Design $(n=388)$

\begin{tabular}{|c|c|c|c|c|}
\hline \multicolumn{5}{|c|}{ eTable 6A Primary and Secondary Outcomes for Fish Oil + Aspirin versus Fish oil + Placebo } \\
\hline Outcomes & $\begin{array}{l}\text { Fish Oil + } \\
\text { Aspirin }\end{array}$ & $\begin{array}{l}\text { Fish oil + } \\
\text { Placebo }\end{array}$ & $\begin{array}{l}\text { Relative risk } \\
(95 \% \mathrm{Cl})\end{array}$ & $\begin{array}{l}\text { P } \\
\text { Value }\end{array}$ \\
\hline $\begin{array}{l}\text { Primary Outcome } \\
\text { AVF failure (composite of thrombosis, AVF } \\
\text { abandonment, and cannulation failure), n/total (\%) }\end{array}$ & $48 / 99(48)$ & $37 / 99(37)$ & $1.30(0.94,1.80)$ & 0.12 \\
\hline $\begin{array}{l}\text { Secondary Outcomes } \\
\text { AVF abandonment within } 12 \text { months, n/total (\%) }\end{array}$ & $26 / 99(26)$ & $15 / 99(15)$ & $1.73(0.98,3.07)$ & 0.06 \\
\hline $\begin{array}{l}\text { Thrombosis of study AVF within } 12 \text { months, n/total } \\
(\%)\end{array}$ & 19/99 (19) & $15 / 99(15)$ & $1.27(0.68,2.35)$ & 0.45 \\
\hline Cannulation failure within 12 months, n/total (\%) & $41 / 99(41)$ & $35 / 99(35)$ & $1.17(0.82,1.67)$ & 0.38 \\
\hline \multicolumn{5}{|c|}{ eTable 6B Primary and Secondary Outcomes for Fish Oil + Aspirin versus Aspirin + Placebo } \\
\hline Outcomes & $\begin{array}{l}\text { Fish Oil + } \\
\text { Aspirin }\end{array}$ & $\begin{array}{l}\text { Placebo + } \\
\text { Aspirin }\end{array}$ & $\begin{array}{l}\text { Relative risk } \\
(95 \% \mathrm{Cl})\end{array}$ & $\begin{array}{l}P \\
\text { Value }\end{array}$ \\
\hline $\begin{array}{l}\text { Primary Outcome } \\
\text { AVF failure (composite of thrombosis, AVF } \\
\text { abandonment, and cannulation failure), n/total (\%) }\end{array}$ & $48 / 99(48)$ & $39 / 95(41)$ & $1.18(0.86,1.62)$ & 0.30 \\
\hline $\begin{array}{l}\text { Secondary Outcomes } \\
\text { AVF abandonment within } 12 \text { months, n/total (\%) }\end{array}$ & $26 / 99(26)$ & $20 / 95(21)$ & $1.25(0.75,2.08)$ & 0.40 \\
\hline $\begin{array}{l}\text { Thrombosis of study AVF within } 12 \text { months, n/total } \\
(\%)\end{array}$ & $19 / 99(19)$ & $19 / 95(20)$ & $0.96(0.54,1.70)$ & 0.89 \\
\hline Cannulation failure within 12 months, n/total (\%) & $41 / 99(41)$ & $32 / 95(34)$ & $1.23(0.85,1.77)$ & 0.27 \\
\hline \multicolumn{5}{|c|}{ eTable 6C Primary and Secondary Outcomes for Fish Oil + Aspirin versus Placebo + Placebo } \\
\hline Outcomes & $\begin{array}{l}\text { Fish Oil + } \\
\text { Aspirin }\end{array}$ & $\begin{array}{l}\text { Placebo + } \\
\text { Placebo }\end{array}$ & $\begin{array}{l}\text { Relative risk } \\
(95 \% \mathrm{Cl})\end{array}$ & $\begin{array}{l}\mathbf{P} \\
\text { Value }\end{array}$ \\
\hline $\begin{array}{l}\text { Primary Outcome } \\
\text { AVF failure (composite of thrombosis, AVF } \\
\text { abandonment, and cannulation failure), n/total (\%) }\end{array}$ & $48 / 99(48)$ & $46 / 95(48)$ & $1.00(0.75,1.34)$ & 0.99 \\
\hline $\begin{array}{l}\text { Secondary Outcomes } \\
\text { AVF abandonment within } 12 \text { months, n/total (\%) }\end{array}$ & $26 / 99(26)$ & $20 / 95(21)$ & $1.25(0.74,2.08)$ & 0.40 \\
\hline $\begin{array}{l}\text { Thrombosis of study AVF within } 12 \text { months, n/total } \\
(\%)\end{array}$ & $19 / 99(19)$ & $20 / 95(21)$ & $0.91(0.52,1.60)$ & 0.75 \\
\hline Cannulation failure within 12 months, n/total (\%) & $41 / 99(41)$ & $39 / 95(41)$ & $1.01(0.72,1.41)$ & 0.96 \\
\hline
\end{tabular}

Abbreviations: AVF - arteriovenous fistula; $\mathrm{Cl}$ - confidence interval

$P$ values for interaction tests: AVF Failure composite $p=0.12$; AVF thrombosis $p=0.45$; AVF abandonment $p=0.06$;

Cannulation failure $\mathrm{p}=0.38$

(C) 2017 American Medical Association. All rights reserved. 
eTable 3: Summary of Missing Data and Single Imputation across Components and the Composite Outcome for $n=536$ participants

\begin{tabular}{|l|c|c|c|c|}
\hline & $\begin{array}{c}\text { AVF } \\
\text { abandonment }\end{array}$ & $\begin{array}{c}\text { AVF } \\
\text { thrombosis }\end{array}$ & $\begin{array}{c}\text { Cannulation } \\
\text { failure }\end{array}$ & $\begin{array}{c}\text { AVF failure } \\
\text { composite }\end{array}$ \\
\hline Missing data & $50(9 \%)$ & $49(9 \%)$ & $111(21 \%)$ & $122(23 \%)$ \\
\hline Fish oil & 28 & 28 & 58 & 62 \\
\hline Placebo & 22 & 21 & 53 & 60 \\
\hline Observed events & 110 & 99 & 168 & 198 \\
\hline $\begin{array}{l}\text { Observed proportion of } \\
\text { events }\end{array}$ & 0.2263 & 0.2033 & 0.3953 & 0.4783 \\
\hline $\begin{array}{l}\text { Imputed number of } \\
\text { events }\end{array}$ & 11 & 10 & 44 & $55^{*}$ \\
\hline Fish oil & 8 & 5 & 26 & $32^{*}$ \\
\hline Placebo & 3 & 5 & 18 & $23^{*}$ \\
\hline
\end{tabular}

*Not directly imputed and dependent on at least one imputed event across the components

(C) 2017 American Medical Association. All rights reserved. 
eTable 4: First Cannulation by Treatment Groups

\begin{tabular}{|l|c|c|c|c|}
\hline Treatment Groups & $\begin{array}{c}\text { Within 12 weeks } \\
\text { of AVF creation, } \\
\mathbf{n}(\%)\end{array}$ & $\begin{array}{c}\mathbf{> 1 2} \text { weeks - 12 } \\
\text { months after } \\
\text { AVF creation, } \mathbf{n} \\
(\%)\end{array}$ & $\begin{array}{c}\text { Not within 12 } \\
\text { months of AVF } \\
\text { creation, } \mathbf{n}(\%)\end{array}$ & $\begin{array}{c}\text { P- } \\
\text { value }\end{array}$ \\
\hline Fish oil $(\mathrm{n}=270)$ & $94(35)$ & $74(27)$ & $102(38)$ & 0.99 \\
\hline Fish oil placebo $(\mathrm{n}=266)$ & $93(35)$ & $72(27)$ & $101(38)$ & \\
\hline Aspirin subset $(\mathrm{n}=194)$ & $66(34)$ & $57(29)$ & $71(37)$ & 0.48 \\
\hline $\begin{array}{l}\text { Aspirin placebo subset } \\
(\mathrm{n}=194)\end{array}$ & $76(39)$ & $48(25)$ & $70(36)$ & \\
\hline
\end{tabular}

Abbreviation: AVF - arteriovenous fistula

(C) 2017 American Medical Association. All rights reserved. 
eTable 5: Months to Hemodialysis Initiation for Pre-dialysis Participants by Treatment Groups

\begin{tabular}{|l|c|c|c|}
\hline Treatment Groups & Median (IQR) & Mean ( \pm SD) & P-value \\
\hline Fish oil $(\mathrm{n}=82)$ & $3.0(1.0,6.0)$ & $4.2(3.5)$ & 0.66 \\
\hline Fish oil placebo $(\mathrm{n}=76)$ & $3.5(2.0,7.0)$ & $4.4(3.3)$ & \\
\hline Aspirin subset $(\mathrm{n}=59)$ & $3.0(1.0,5.0)$ & $4.0(3.5)$ & 0.41 \\
\hline Aspirin placebo subset $(\mathrm{n}=53)$ & $3.0(1.0,8.0)$ & $4.6(3.7)$ & \\
\hline
\end{tabular}

Abbreviations: IQR - interquartile range; SD - standard deviation

(C) 2017 American Medical Association. All rights reserved. 


\section{eTable 6: Timing of First Renal Replacement Therapy for Pre-dialysis Participants by Treatment Groups}

\begin{tabular}{|l|l|l|l|l|l|}
\hline $\begin{array}{l}\text { Time } \\
\text { point of first RRT }\end{array}$ & $\begin{array}{l}\text { RRT } \\
\text { modality }\end{array}$ & $\begin{array}{l}\text { Fish oil, n } \\
(\mathbf{\%})\end{array}$ & $\begin{array}{l}\text { Fish oil } \\
\text { Placebo, } \mathbf{n} \\
(\%)\end{array}$ & $\begin{array}{l}\text { Aspirin, n } \\
\text { (\%) }\end{array}$ & $\begin{array}{l}\text { Aspirin } \\
\text { Placebo, } \mathbf{n} \\
\text { (\%) }\end{array}$ \\
\hline $\begin{array}{l}\text { Within first 12 } \\
\text { weeks of AVF } \\
\text { creation }\end{array}$ & HD & $43(31)$ & $38(28)$ & $32(32)$ & $28(29)$ \\
\hline & PD & $10(7)$ & $7(5)$ & $4(4)$ & $7(7)$ \\
\hline & Transplant & $0(0)$ & $1(1)$ & $0(0)$ & $1(1)$ \\
\hline $\begin{array}{l}>12 \text { weeks - 12 } \\
\text { months after AVF } \\
\text { creation }\end{array}$ & HD & $39(28)$ & $38(28)$ & $27(27)$ & $25(26)$ \\
\hline & & & & \\
\hline & PD & $5(4)$ & $5(4)$ & $3(3)$ & $4(4)$ \\
\hline
\end{tabular}

(C) 2017 American Medical Association. All rights reserved. 
eFigure 1: Study Schema

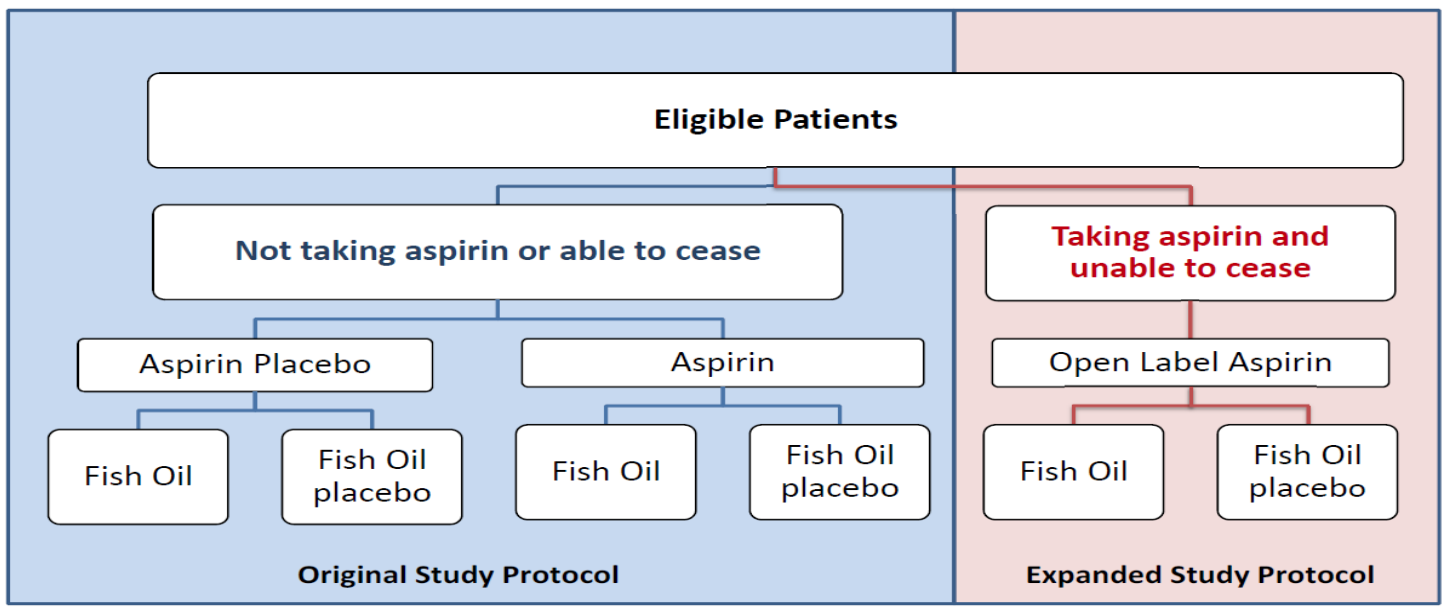

(C) 2017 American Medical Association. All rights reserved. 


\section{eFigure 2: Forest Plot for Sensitivity Analysis Fish oil versus Placebo (A) and Aspirin versus Placebo (B)}

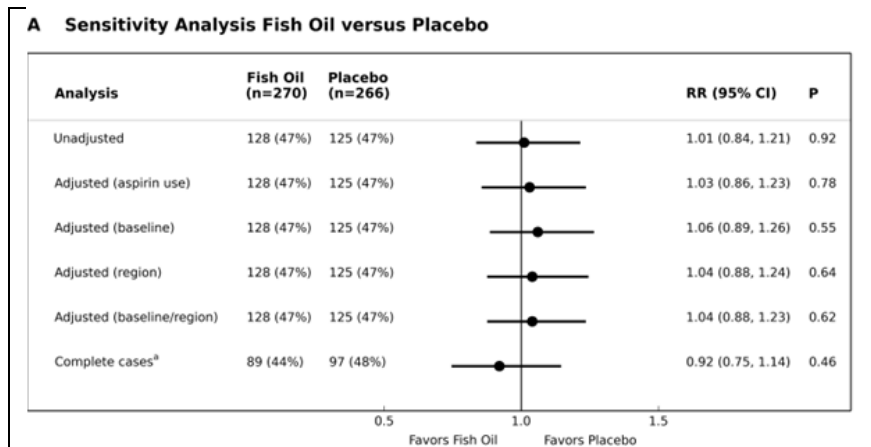

A: ${ }^{a}$ The complete case analysis is adjusted for aspirin use; $n=201$ for participants taking fish oil and $n=201$ for participants taking placebo.
B Sensitivity Analysis Aspirin versus Placebo

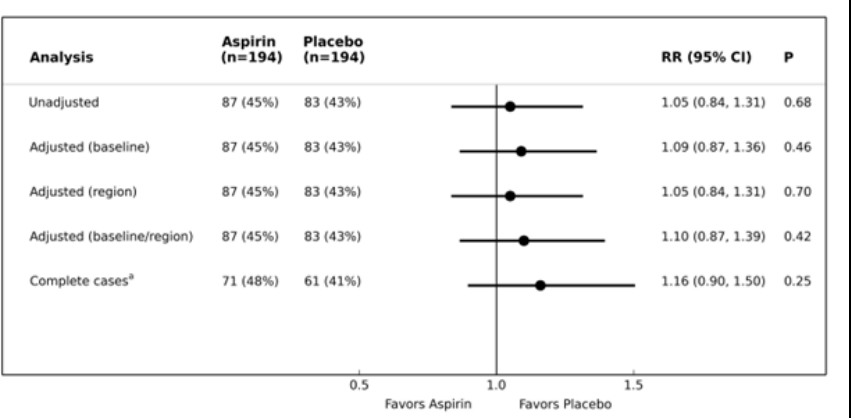

B: ${ }^{a}$ The complete case analysis includes 149 participants taking aspirin 149 participants taking placebo.

Pre-specified baseline characteristics included planned AVF site (lower arm, upper arm), diabetes mellitus, age group (quartiles), cardiovascular comorbidity, including any one or more of peripheral vascular disease, ischaemic heart disease and cerebrovascular accidents, and renal replacement therapy at baseline (no [pre-dialysis/transplant], yes [hemodialysis or peritoneal dialysis]). Regions included Australia and New Zealand, Malaysia and the United Kingdom. Abbreviations: $\mathrm{Cl}$ - confidence interval; $\mathrm{RR}$-relative risk ratio 
eFigure 3: Erythrocyte Fatty Acid Composition by Study Visit and Treatment: Eicosapentaenoic Acid (EPA; Panel A), Docosahexaenoic Acid (DHA; Panel B), and EPA and DHA Combined (Panel C)
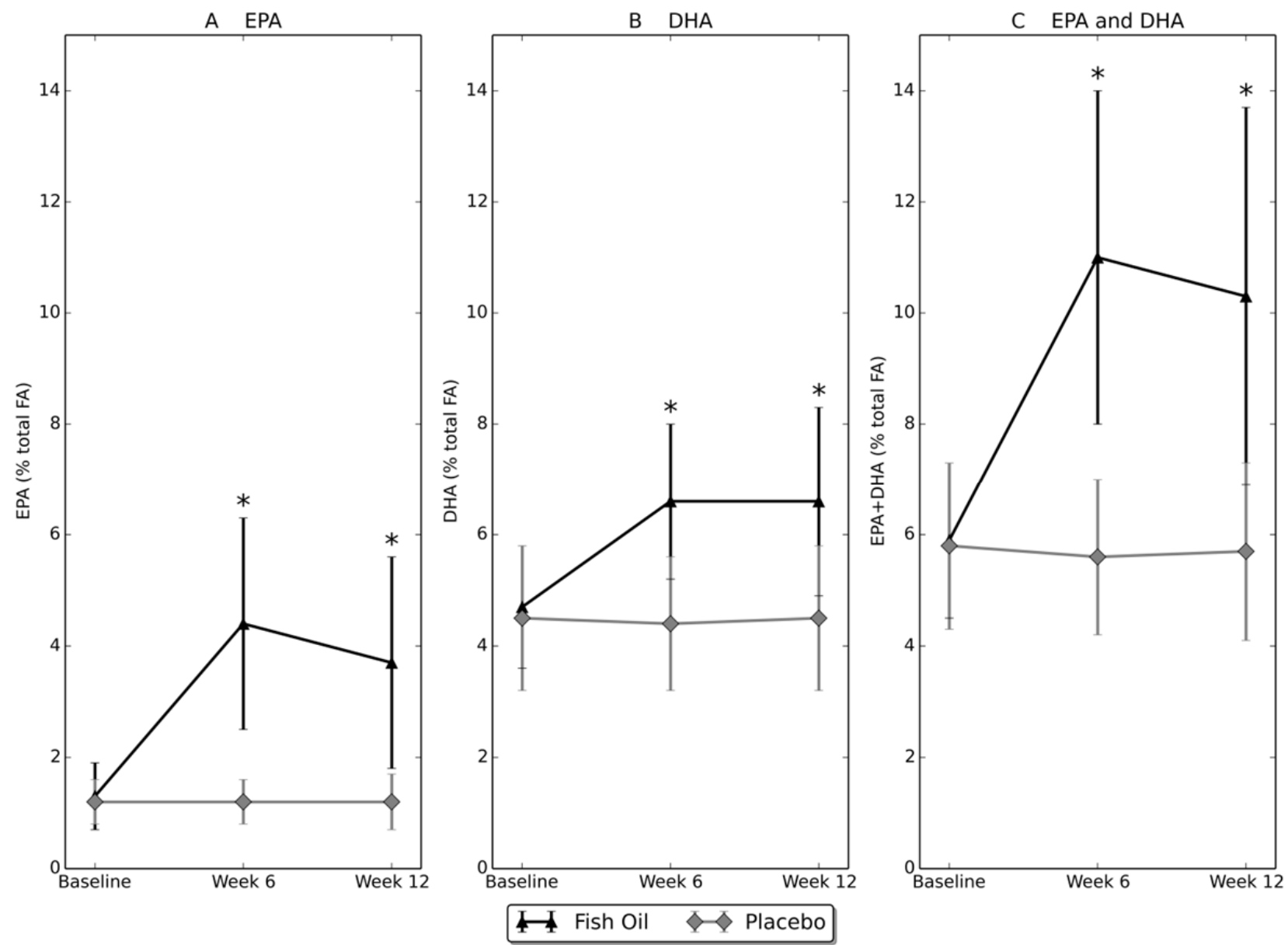

Erythrocyte fatty acids composition of Australian and New Zealand participants treated with fish oil (triangle) or placebo (square) at baseline, week 6 and 12 of fish oil supplementation shown as mean percentage of total erythrocyte fatty acids and standard deviation (error bars) for EPA (Panel A), DHA (Panel B), and EPA+DHA (Panel C).

*The difference in erythrocyte fatty acid composition at study visits 6 and 12 for Panel A-C was statistically significant between fish oil and placebo treated participants $(\mathrm{p}<0.0001)$.

Abbreviations: DHA - Docosahexaenoic acid; EPA -Eicosapentaenoic acid; FA - Fatty acids. 